\title{
Glucagon-like peptide 1 (GLP-1)
}

Mueller, T. D.; Finan, B.; Bloom, S. R.; D'Alessio, D.; Drucker, D. J.; Flatt, P. R.; Fritsche, A.; Gribble, F.; Grill, H. J.; Habener, J. F.; Holst, J. J.; Langhans, W.; Meier, J. J.; Nauck, M. A.; Perez-Tilve, D.; Pocai, A.; Reimann, F.; Sandoval, D. A.; Schwartz, T. W.; Seeley, R. J.; Stemmer, K.; Tang-Christensen, M.; Woods, S. C.; DiMarchi, R. D.; Tschoep, M. H. Published in:

Molecular Metabolism

DOI:

10.1016/j.molmet.2019.09.010

Publication date:

2019

Document version

Publisher's PDF, also known as Version of record

Document license:

CC BY-NC-ND

Citation for published version (APA):

Mueller, T. D., Finan, B., Bloom, S. R., D'Alessio, D., Drucker, D. J., Flatt, P. R., Fritsche, A., Gribble, F., Grill, H. J., Habener, J. F., Holst, J. J., Langhans, W., Meier, J. J., Nauck, M. A., Perez-Tilve, D., Pocai, A., Reimann, F., Sandoval, D. A., Schwartz, T. W., ... Tschoep, M. H. (2019). Glucagon-like peptide 1 (GLP-1). Molecular Metabolism, 30, 72-130. https://doi.org/10.1016/j.molmet.2019.09.010 


\title{
Glucagon-like peptide 1 (GLP-1)
}

\author{
T.D. Müller ${ }^{1,2,3, *}$, B. Finan ${ }^{4}$, S.R. Bloom ${ }^{5}$, D. D’Alessio ${ }^{6}$, D.J. Drucker ${ }^{7}$, P.R. Flatt ${ }^{8}$, A. Fritsche ${ }^{2,9,10}$, \\ F. Gribble ${ }^{11}$, H.J. Grill ${ }^{12}$, J.F. Habener ${ }^{13}$, J.J. Holst ${ }^{14}$, W. Langhans ${ }^{15}$, J.J. Meier ${ }^{16}$, M.A. Nauck ${ }^{17}$, \\ D. Perez-Tilve ${ }^{18}$, A. Pocai ${ }^{19}$, F. Reimann ${ }^{11}$, D.A. Sandoval ${ }^{20}$, T.W. Schwartz ${ }^{21,22}$, R.J. Seeley ${ }^{20}$, \\ K. Stemmer ${ }^{1,2}$, M. Tang-Christensen ${ }^{23}$, S.C. Woods ${ }^{24}$, R.D. DiMarchi ${ }^{4,25}$, M.H. Tschöp ${ }^{2,26,27}$
}

\section{ABSTRACT}

Background: The glucagon-like peptide-1 (GLP-1) is a multifaceted hormone with broad pharmacological potential. Among the numerous metabolic effects of GLP-1 are the glucose-dependent stimulation of insulin secretion, decrease of gastric emptying, inhibition of food intake, increase of natriuresis and diuresis, and modulation of rodent $\beta$-cell proliferation. GLP-1 also has cardio- and neuroprotective effects, decreases inflammation and apoptosis, and has implications for learning and memory, reward behavior, and palatability. Biochemically modified for enhanced potency and sustained action, GLP-1 receptor agonists are successfully in clinical use for the treatment of type-2 diabetes, and several GLP-1-based pharmacotherapies are in clinical evaluation for the treatment of obesity.

Scope of review: In this review, we provide a detailed overview on the multifaceted nature of GLP-1 and its pharmacology and discuss its therapeutic implications on various diseases.

Major conclusions: Since its discovery, GLP-1 has emerged as a pleiotropic hormone with a myriad of metabolic functions that go well beyond its classical identification as an incretin hormone. The numerous beneficial effects of GLP-1 render this hormone an interesting candidate for the development of pharmacotherapies to treat obesity, diabetes, and neurodegenerative disorders

(c) 2019 The Authors. Published by Elsevier GmbH. This is an open access article under the CC BY-NC-ND license (http://creativecommons.org/licenses/by-nc-nd/4.0/).

Keywords GLP-1; Insulin; Glucagon; Diabetes; Obesity; Incretin

\section{FROM THE DISCOVERY OF INSULIN TO THE DISCOVERY OF GLP-1}

Maintenance of adequate glucose metabolism is a prerequisite for human health, and pathological failure to buffer against prolonged episodes of hypo- and/or hyperglycemia can result in severe microvascular disease, metabolic damage, coma, and death. Unsurprisingly, before the discovery and commercialization of insulin in the 1920's, juvenile-onset diabetes, with its paucity of endogenous insulin, was a disease with only a few years between a patient's diagnosis and premature demise. The discovery of insulin and its ability to lower blood glucose transformed juvenile-onset (type-1) diabetes from a fatal to a manageable disease. However, early on, it was noted that insulin derived from pancreatic extracts [1] or as crude insulin preparations [2] sometimes first elevated blood glucose and then later decreased blood glucose levels. The increase in blood glucose, which peaked around 20 min after the administration, was believed to be caused by a toxic fraction resulting from suboptimal insulin purification [2]. The same

\begin{abstract}
${ }^{1}$ Institute for Diabetes and Obesity, Helmholtz Diabetes Center, Helmholtz Zentrum München, German Research Center for Environmental Health (GmbH), Neuherberg, Germany ${ }^{2}$ German Center for Diabetes Research (DZD), Neuherberg, Germany ${ }^{3}$ Department of Pharmacology and Experimental Therapy, Institute of Experimental and Clinical Pharmacology and Toxicology, Eberhard Karls University Hospitals and Clinics, Tübingen, Germany ${ }^{4}$ Novo Nordisk Research Center Indianapolis, Indianapolis, IN, USA ${ }^{5}$ Division of Diabetes, Endocrinology and Metabolism, Imperial College London, London, UK ${ }^{6}$ Division of Endocrinology, Duke University Medical Center, Durham, NC, USA ${ }^{7}$ The Department of Medicine, Lunenfeld-Tanenbaum Research Institute, Mt. Sinai Hospital, University of Toronto, Ontario, M5G1X5, Canada ${ }^{8}$ SAAD Centre for Pharmacy \& Diabetes, Ulster University, Coleraine, Northern Ireland, UK ${ }^{9}$ Institute for Diabetes Research and Metabolic Diseases of the Helmholtz Center Munich at the University of Tübingen, Tübingen, Germany ${ }^{10}$ Division of Endocrinology, Diabetology, Vascular Disease, Nephrology and Clinical Chemistry, Department of Internal Medicine, University of Tübingen, Tübingen, Germany ${ }^{11}$ Metabolic Research Laboratories and Medical Research Council Metabolic Diseases Unit, Wellcome Trust-Medical Research Council, Institute of Metabolic Science, Addenbrooke's Hospital, University of Cambridge, Cambridge, CB2 0QQ, UK ${ }^{12}$ Institute of Diabetes, Obesity and Metabolism, Department of Psychology, University of Pennsylvania, Philadelphia, PA, 19104, USA ${ }^{13}$ Laboratory of Molecular Endocrinology, Massachusetts General Hospital, Harvard University, Boston, MA, USA ${ }^{14}$ Novo Nordisk Foundation Center for Basic Metabolic Research, Department of Biomedical Sciences, University of Copenhagen, Copenhagen, Denmark ${ }^{15}$ Physiology and Behavior Laboratory, ETH Zurich, Schwerzenbach, Switzerland ${ }^{16}$ Diabetes Division, St Josef Hospital, Ruhr-University Bochum, Bochum, Germany ${ }^{17}$ Diabetes Center Bochum-Hattingen, St Josef Hospital (Ruhr-Universität Bochum), Bochum, Germany ${ }^{18}$ Department of Internal Medicine, University of CincinnatiCollege of Medicine, Cincinnati, OH, USA ${ }^{19}$ Cardiovascular \& ImmunoMetabolism, Janssen Research \& Development, Welsh and McKean Roads, Spring House, PA, 19477, USA ${ }^{20}$ Department of Surgery, University of Michigan Medical School, Ann Arbor, MI, USA ${ }^{21}$ Novo Nordisk Foundation Center for Basic Metabolic Research, University of Copenhagen, DL-2200, Copenhagen, Denmark ${ }^{22}$ Department of Biomedical Sciences, University of Copenhagen, DK-2200, Copenhagen, Denmark ${ }^{23}$ Obesity Research, Global Drug Discovery, Novo Nordisk A/S, Måløv, Denmark ${ }^{24}$ Department of Psychiatry and Behavioral Neuroscience, University of Cincinnati, Cincinnati, OH, USA ${ }^{25}$ Department of Chemistry, Indiana University, Bloomington, IN, USA ${ }^{26}$ Division of Metabolic Diseases, Department of Medicine, Technische Universität München, Munich, Germany ${ }^{27}$ Helmholtz Zentrum München, German Research Center for Environmental Health (GmbH), Neuherberg, Germany
\end{abstract}

*Corresponding author. Institute for Diabetes and Obesity, Helmholtz Diabetes Center, Helmholtz Zentrum München, German Research Center for Environmental Health $(\mathrm{GmbH})$, Neuherberg, Germany. E-mail: timo.mueller@helmholtz-muenchen.de (T.D. Müller).

Received July 17, 2019 • Revision received September 10, 2019 • Accepted September 22, 2019 • Available online 30 September 2019

https://doi.org/10.1016/j.molmet.2019.09.010 
toxic fraction was thought to be responsible for local skin irritations and abscesses that were sometimes observed in patients treated with these formulations [2]. These observations spurred efforts to optimize the isolation and purification of insulin from tissue homogenates.

Aiming to develop a fast and inexpensive method for commercial insulin purification, in 1923, Charles Kimball and John Murlin precipitated a pancreatic fraction that, after evaporation and reconstitution in water, had a robust hyperglycemic effect when injected into rabbits and dogs [3]. Because the fraction was incapable of decreasing blood glucose, Kimball and Murlin hypothesized that the hyperglycemic effect resulted from a secreted factor, one that antagonizes insulin's hypoglycemic effect. The factor was named 'the glucose agonist', or "glucagon" [3]. Over the subsequent decades, substantial research efforts were directed toward unravelling the molecular underpinnings of glucose regulation by the two opposing pancreatic hormones (as reviewed in $[4,5])$. Among the numerous major discoveries made in this regard was the finding that the hyperglycemic effect of glucagon resides in its ability to act in the liver to stimulate glycogenolysis and gluconeogenesis [6-12] despite its seemingly paradoxical ability to stimulate insulin secretion in humans reported by Ellis Samols in 1965 [13]. In the early 1950's, Earl Sutherland and Christian de Duve demonstrated that pancreatic glucagon production was abolished when the function of islet $\alpha$-cells was compromised by treatment with either cobalt or synthalin $A$ but was preserved upon alloxan-induced impairment of the exocrine pancreatic acinar and islet $\beta$-cells $[14,15]$. Subsequent histological studies by Claes Hellerstrom and Bo Hellman divided $\alpha$-cells into $\alpha_{1}$-and $\alpha_{2}$-subtypes, but it was not until the early 1960 s that convincing evidence was provided that $\alpha_{2}$-cells were the source of glucagon [16]. This conclusion was based on suppression of $\alpha_{2}$-cell numbers in rats and guinea pigs by administration of exogenous glucagon $[17,18]$ plus the strong staining in $\alpha_{2}$-cells for tryptophan, which is an amino acid present in glucagon. Later, $\alpha_{1}$-cells were shown by Bo Hellman and Ake Lernmark to be the source of an inhibitor of insulin secretion [19]. This was eventually established as somatostatin. In 1959, Roger Unger generated and characterized the first glucagondetecting antibody and thus paved the path to the development of the first radioimmunoassays (RIA) to detect glucagon in blood and tissue samples [20]. It was subsequently found by Ellis Samols and Vincent Marks in 1966 [21] and confirmed by others that glucagon-like immunoreactivity was also present in extra-pancreatic tissues, in particular in the intestine [22-27]. Notably, the circulating glucagonlike material was also detected in 1967 by Samols and Marks in pancreatectomized humans [21] and in the following year by Roger Unger and Isabel Valverde in dogs [27]. This eliminated the pancreas as the origin of this glucagon-like immunoreactivity. Unger then demonstrated in 1968 that intraduodenal, but not intravenous, glucose administration increases circulating glucagon-like immunoreactivity [27], implying that the intestine secretes this glucagon-like material. The intestinal glucagon-like material was heterogeneous, comprising several fractions of different molecular size and with apparently distinct biological actions relative to pancreatic-derived glucagon $[27,28]$. The intestinal glucagon-immunoreactive material did not induce hyperglycemia when injected into dogs and was devoid of glycogenolytic effects in the isolated perfused rat liver [27].

However, analogous to the effects of glucagon, the intestinal material stimulated the release of insulin, suggesting that the intestinal glucagon-like fractions are either different substances or different forms of pancreatic glucagon [27]. Further corroborating the distinct nature of the intestinal and pancreatic glucagon-like material, immunocytochemical studies revealed that the intestinal cells that stained positive for the glucagon antibody differed from the pancreatic glucagon-producing $\alpha$-cells in terms of their morphology and ultrastructure [29], classifying these intestinal cells as "L-cells" [30]. Glucagon-like immunoreactive material of larger size than of glucagon was subsequently detected in the pancreas of dogs [31] as well as in islets isolated from birds [32] and guinea pigs [33]. Collectively, these studies suggested that glucagon might originate from a larger precursor molecule that is post-translationally cleaved into several fractions of distinct size and with different functions. Immunoprecipitation analysis of rat pancreatic islets identified this precursor as an 18,000 molecular weight (MW) protein, now classified as proglucagon [34]. In the pancreas, proglucagon was found to be cleaved to produce two fragments, mature glucagon and a 10,000-MW second protein. This latter protein lacked the glucagon sequence and was named major proglucagon fragment (MPGF) [34,35] (Figure 1).

In the early 1980s, it was established that the major form of the intestinal glucagon-immunoreactive material, a peptide designated glicentin, contained the full glucagon sequence [36-40], and glicentin was proposed to represent at least a fragment of proglucagon, because it was also identified in the pancreas [41]. Although glicentin was initially thought to comprise $\sim 100$ amino acids, its purification from porcine intestine established glicentin as a 69-amino acid peptide [39]. Thus, intestinal proglucagon is cleaved into distinct fractions that are different from those derived in the pancreas. In 1982, a smaller intestinal form was identified as a 37-amino acid peptide containing the full 29-amino acid sequence of glucagon with 8 additional amino acids on its C-terminal end [36], the same as found in the C-terminus of glicentin. Based on its potency to act on oxyntic glands, the 37amino acid peptide was named oxyntomodulin [42] (Figure 1). Thus, it appeared that proglucagon undergoes a tissue-specific, differential processing leading to the formation of glicentin and oxyntomodulin in the gut and to glucagon plus the $\mathrm{N}$-terminal fragment of glicentin in the pancreas [34,43] (Figure 1). By using a method that allows the prediction of a protein sequence through decoding of recombinant cDNA clones, at the beginning of the 1980s Joel Habener established that a different glucagon-related peptide is encoded within the anglerfish preproglucagon cDNA [44-46]. Two glucagon-related peptides were subsequently identified in the rat [47,48], hamster [49], bovine [50], and human [51] proglucagon sequence. The two hypothetical peptides were designated glucagon-like peptides 1 and 2 (GLP-1 and GLP-2) [49] (Figure 1). Radioimmunoassays in tissues from rats [52], pigs [53], and humans [54] then established a distinct profile of proglucagon-derived peptides (PGDPs) in the pancreas and the intestine with production of glucagon and one large fragment (major proglucagon fragment; MPGF) in the pancreas and liberation of smaller GLP-1 immunoreactive peptides in the intestine.

\section{IDENTIFICATION OF GIP AND GLP-1 AS GASTROINTESTINAL INSULINOTROPIC HORMONES}

In 1902, Ernest Bayliss and William Starling identified a substance that is produced in and secreted from the epithelial cells of the duodenum in response to the contact of these cells with acidic chyme [55]. Bayliss and Starling noted that the substance, after being released into the circulation, stimulates the pancreas to secrete pancreatic juice; they named the substance secretin, thereby identifying the first gastrointestinal hormone [55]. In 1906, Benjamin Moore reported that the glucosuria of diabetic patients could be ameliorated by repeated oral administration of a pigderived intestinal mucosal homogenate [55]. Influenced by the work of Bayliss and Starling, Moore hypothesized that the intestinal mucosa membrane produces a substance of the nature of a hormone that decreases blood glucose via its stimulatory action on the pancreas [55]. 


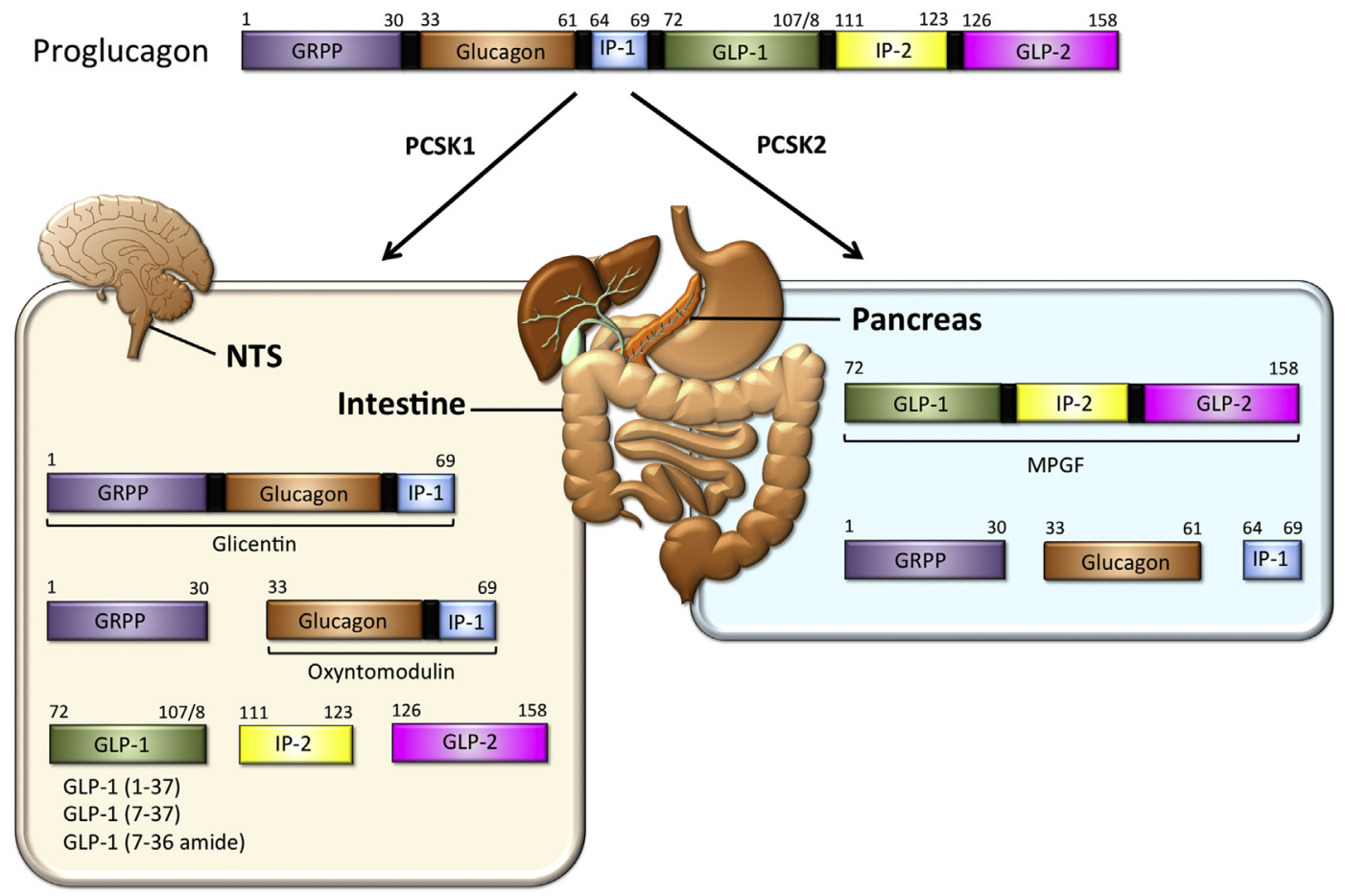

Figure 1: Schematic on the tissue-selective processing of proglucagon. PCSK1: prohormone convertase 1/3; PCSK2: prohormone convertase 1/3; NTS: nucleus tractus solitarii; GRPP: glicentin-related polypeptide; IP-1 intervening peptide-1; IP-2: intervening peptide-2, MPGF: major proglucagon fragment; GLP-1: glucagon-like peptide-1; GLP-2: glucagon-like peptide-2. See text for further explanations.

Supporting Moore's hypothesis, in 1929, Edgard Zunz and Jean LaBarre isolated a fraction from gut extracts that decreased blood glucose in experimental animals [56]. Assuming that the hypoglycemic effect of this fraction resides in its ability to stimulate the endocrine pancreas to secrete insulin, the factor was named incretin $[56,57]$.

In the following years, research directed towards identification of insulinotropic intestinal factor(s) was hampered by difficulties in quantifying insulin from tissue and blood samples. Once the first insulin radioimmunoassays (RIA) became available in the 1960's [58,59], the insulinotropic action of intestinal mucosa extracts was confirmed in healthy humans and was demonstrated to be absent in individuals with type-1 diabetes [60]. Several nearly simultaneous reports then demonstrated that glucose-stimulation of insulin secretion was much greater when glucose was given orally as compared to parenterally [61,62]. Greater insulinotropic action of oral glucose relative to its peripheral administration was demonstrated for both normal weight and obese individuals [63] and henceforth became known as the incretin effect [64,65]. In 1965, Ellis Samols and colleagues had already hypothesized that the intestinal glucagon-like material might somehow be related to the incretin effect [66]. Between 1969 and 1971, work by John Brown and Raymond Pederson, together with the peptide chemistry expertise of Erik Jorpes and Viktor Mutt [67], led to the identification of the gastric inhibitory polypeptide (GIP), a 42-amino acid polypeptide that was named based on its potency to suppress gastric motility and gastric acid secretion [68-70]. In 1973, John Dupré demonstrated in healthy human volunteers that GIP, when given intravenously at near-physiological doses together with glucose, potentiates immunoreactive insulin and improves glucose tolerance, thus identifying GIP as a contributor to the incretin effect [71]. The insulinotropic action of GIP was subsequently demonstrated to be due to direct action on the pancreas, where it enhances glucose-stimulation of insulin secretion, as demonstrated in isolated rat islets [72-75], and in the perfused pancreas of dogs [76] and humans [77].

Work from Werner Creutzfeldt's laboratory in 1983 demonstrated that removal of GIP from gut extracts by immunoprecipitation diminishes the incretin effect by less than $50 \%$ [78]. Studies in humans with resection of different parts of the small intestine demonstrated that the incretin effect and the GIP response to oral glucose did not correlate the incretin effect was better correlated with preservation of the ileum [79]. Because glucagon was known to stimulate insulin secretion [13], it was natural to look at the newly identified glucagon-like peptides that possessed $\sim 50 \%$ sequence homology to glucagon. Indeed, the glucagon-like peptide that was identified in the anglerfish, as well as the mammalian proglucagon CDNA and both resembled the GIP sequence, suggesting that these proglucagon products might have insulinotropic effects [44-46]. Neither GLP-1 (1-36amide) nor GLP-2, peptide fragments corresponding to the predicted structures from the gene sequence, were active on insulin secretion, but a truncated version of GLP-1 extractable from human and porcine gut was subsequently found to enhance insulin secretion in various experimental models [80-82] and, ultimately, in human studies, i.e. GLP-1 was identified as a potential incretin hormone [83].

\section{TRANSCRIPTIONAL REGULATION OF PREPROGLUCAGON (GCG)}

Preproglucagon (Gcg) is expressed in pancreatic $\alpha$-cells, in enteroendocrine L-cells throughout the gut, predominantly in the distal ileum and colon, and in a population of neurons in the nucleus tractus solitarii (NTS) of the brainstem [52,84-89]. Diphtheria toxin-induced ablation of preproglucagon-positive neurons in the NTS 
demonstrated that this small population of neurons is the primary source of endogenous GLP-1 in the brain [90].

Cleavage sites within the proglucagon molecule together with expression of specific prohormone convertase enzymes determines which smaller peptide molecules/hormones are formed, including glicentin (aa 1-69), glicentin-related pancreatic polypeptide (GRPP; aa 1-30), glucagon (aa 33-61), oxyntomodulin (OXM; aa 33-69), the major proglucagon fragment (MPGF; aa 72-158), and the glucagonlike peptides 1 (GLP-1; aa 72-107/108) and 2 (GLP-2; aa 126158) (Figure 1) [51-53,91]. Several of the PGDPs have important and well-defined (pharmacological) effects on systemic metabolism, including the modulation of food intake and satiety (GLP-1, glucagon, oxyntomodulin), regulation of fluid homeostasis (water intake and urine excretion) (GLP-1) [92], thermogenesis (glucagon), lipid metabolism (GLP-1, glucagon, GLP-2), gastrointestinal motility (glucagon, GLP-1, GLP-2), and gastric emptying (glucagon, GLP-1, GLP-2). Glucagon released from pancreatic $\alpha$-cells and GLP-1 released from intestinal Lcells have opposing effects on blood glucose. Therefore, the expression and cleavage of proglucagon and secretion of the various PGDPs must be precisely controlled in a cell-specific process.

The expression of Gcg in the pancreas, intestine, and brain is under the control of a single promoter and is initiated from an identical transcription start codon (Figure 2). The rodent Gcg promoter, along with its adjacent DNA control/enhancer elements, is located within the
$2.5 \mathrm{~kb} 5^{\prime}$-flanking region of the Gcg transcription start [89,93,94]. In rodents, the $\sim 1.3 \mathrm{~kb} 5^{\prime}$-flanking sequence is sufficient to direct transgene expression to Gcg + cells in the brain and the pancreas [95] but extension of this region to include $\sim 2.5 \mathrm{~kb}$ is required to target Gcg + cells in the intestine [96], including evolutionarily preserved sequences in the first intron [97].

The cell-specific expression of Gcg is orchestrated by a series of homeodomain proteins that bind to specific cis-acting elements in the Gcg promoter and/or enhancer region to either stimulate or inhibit Gcg promoter activity [94,98-100]. The rat Gcg promoter comprises at least 5 cis-acting elements $\left(G_{1}-G_{5}\right)$ plus a cAMP response element (CRE), all of which are located within the $2.5 \mathrm{~kb}$ region upstream of the Gcg transcription start $[89,94,101-103]$. In $\alpha$-cells, the TATA box, as well as the adjacent G1 and G4 elements, represent the minimal promoter which is essential for Gcg expression while the elements G5, G2, G3, and CRE represent a more distal located enhancer region [94,101-103] (Figure 2).

Signaling events leading to the stimulation of Gcg expression in $\alpha$-cells include heterodimerization of the transcription factor paired box protein 6 (Pax6) with cellular muscular aponeurotic fibrosarcoma (c-Maf), MAF bZIP transcription factor B (MafB) or caudal type homeobox 2/3 (Cdx2/ 3 ), and consequent binding of these heterodimers to the G1 element (Figure 2) [98,104-106]. Pax6 can also bind to the G3 element [100], and it plays a key role in regulating Gcg expression and $\alpha$-cell
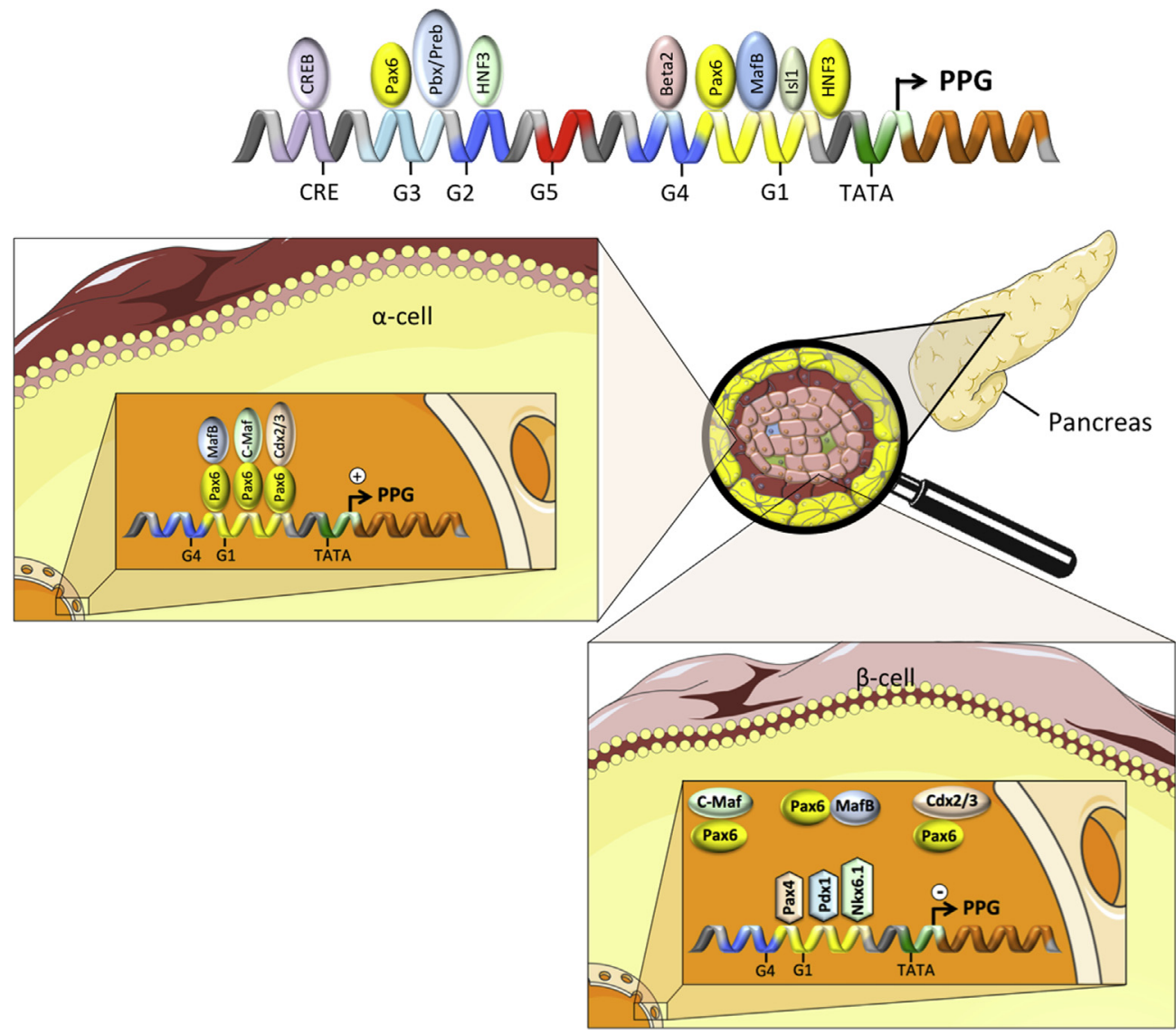

Figure 2: Schematic on the tissue-selective processing of proglucagon in the pancreatic islets. Schematic on the transcriptional regulation of preproglucagon (PPG) in the pancreatic islets. Pax6: paired box 6; CDX2/3: caudal type homeobox 2/3; MafB: MAF bZIP transcription factor B; cMaf: c-Maf inducing protein; NKX2.1: NK2 homeobox 1; PDX1: pancreatic and duodenal homeobox 1; Pax4: paired box 4; CRE: CAMP response element; CREB: cAMP response element binding protein; PPG: preproglucagon; HNF3: hepatocyte nuclear factor 3; IsI1: ISL LIM homeobox 1; Preb: prolactin element binding. For further explanations, please see text. 
development, because mice lacking Pax6 fail to produce glucagonproducing $\alpha$-cells [107]. Pax6 also stimulates Gcg expression in the enteroendocrine cells of the gastrointestinal epithelium [108]. Mice homozygous for a dominant negative Pax6 mutation (SEYNeu) have repressed Gcg expression in enteroendocrine cells in the small and large bowel and absence of immunoreactive GLP-1 and GLP-2 [109]. Further supporting the role of Pax6 in regulating intestinal $\mathrm{Gcg}$ expression, adenoviral overexpression of Pax6 enhances Gcg promoter activity and Gcg expression in intestinal enteroendocrine cells such as the secretin tumor cell line-1 (STC-1) and cells derived from colonic tumors of transgenic mice expressing large $T$ antigen under the control of the proglucagon promoter (GLUTag cells) [108].

Other transcriptional mechanisms regulating Gcg expression in $\alpha$-cells include interaction of $\mathrm{Cdx} 2 / 3$, POU domain transcription factor brain 4 (Brn-4), hepatocyte nuclear factor 3 alpha (HNF3 $\alpha$; a.k.a. Foxa1), hepatocyte nuclear factor 3 beta (HNF3 $\beta$; a.k.a. Foxa2), paired box protein 2 (Pax2), neuronal differentiation factor 1/beta 2 (NeuroD/ Beta2), and basic helix-loop-helix transcription factor E47 with the G1, G2, G3, or G4 elements (Figure 2) [100,102,104,106,110-118]. Emphasizing their role in regulating glycemia, mice lacking Foxa1 or Foxa2 die shortly after birth due to severe hypoglycemia and substantial reduction in Gcg mRNA levels in the pancreas [119,120]. Notably, $\alpha$-cell development is impaired in mice lacking Foxa2 but is normal in mice lacking Foxa1 [119,120]. These data suggest that Foxa1 affects glucagon levels via its action on the Gcg promoter, while Foxa2, in addition to modulating Gcg promoter activity, affects $\alpha$-cell differentiation. Interestingly, $\beta$-cell specific deletion of Foxa2 also results in postnatal death due to severe hypoglycemia, but the low glucose level in Foxa2-negative mice seems to be caused by hyperinsulinemia rather than by changes in Gcg expression [121]. The LIM homeobox protein 1 (Isl1) is ubiquitously expressed in mature endocrine cells of the pancreas [122], and its ablation in mice results in failure to develop any pancreatic endocrine cell type [123]. In Chinese Hamster insulinoma InR1-G9 cells, Isl1 enhances the activity of the Gcg promoter [118]. Isl1 also interacts with the enhancer region of the genes encoding for insulin [124] and somatostatin [125], stimulating their transcription. In islet and intestinal endocrine cell lines, $\mathrm{Cdx} 2 / 3$ (the same protein is characterized as $\mathrm{Cdx} 2$ in mice and $\mathrm{Cdx} 3$ in hamsters) activates $G c g$ gene transcription via binding to the $\mathrm{G} 1$ element of the $\mathrm{Gcg}$ promoter [98]. Overexpression of Cdx2 in $\alpha$-cell InR1-G9 cells accordingly increases Gcg expression [113].

In both $\alpha$-cells and intestinal L-cells, Gcg expression is controlled by certain homeodomain proteins $[94,98,100]$ and by cAMP-activation of protein kinase $A(P K A)$, as demonstrated in primary rat intestinal cultures [126], isolated pancreatic cell lines [127,128] and enteroendocrine GLUTag and STC-1 cell lines $[129,130]$. In STC-1 cells, disruption of the CRE element in the Gcg enhancer only partially blunts PKA-stimulation of Gcg expression $[130,131]$. This suggests that PKA also affects Gcg transcription via CRE-independent mechanisms. Consistent with this, certain effectors of the Wnt pathway, including lithium and $\beta$-catenin, enhance Gcg expression in intestinal but not pancreas-derived cell lines, supposedly via inhibition of the glycogen synthase kinase-3beta (GSK-3beta) [132,133]. PKA has been demonstrated to inhibit GSK-3beta $[134,135]$, indicating that the CREindependent PKA stimulation of the Gcg promoter might be mediated via negative regulation of the Wnt pathway $[132,133]$. Other factors stimulating Gcg expression in the intestine include protein hydrolysates [101] and insulin [133]. Insulin stimulation of intestinal Gcg promoter activity and of GLP-1 secretion [133] is noteworthy because insulin inhibits glucagon production and secretion in isolated rat islets [136] and in hamster islet InR1-G9 cells [137]. While the mechanism underlying insulin inhibition of pancreatic glucagon production warrants further clarification, it is thought to be achieved by through transcriptional mechanisms via an insulin-responsive element (IRE) in the Gcg promoter of the $\alpha$-cells [138].

Notably, whereas $\mathrm{Gcg}$ is robustly expressed in $\alpha$-cells, its expression is suppressed in $\beta$-cells through binding of pancreatic and duodenal homeobox 1 (Pdx1), Pax4, and homeobox protein Nkx6.1 to the G1 element, thereby competitively preventing the binding of Pax6/Maf heterodimers to the G1 element (Figure 2) $[100,139,140]$. While substantial evidence supports a role of $\mathrm{Pdx} 1$ in the negative regulation of Gcg expression, Pdx1 immunoreactivity has also been demonstrated in some Gcg expressing L-cells [141]. Overexpression of Pdx1 alone is insufficient to block Gcg expression in $\alpha$-cell cultures ( $\alpha \mathrm{TC}-1$ cells), isolated murine islets, or GLUTag enteroendocrine cells [142]. These data collectively suggest that $\mathrm{Pdx} 1$ requires interaction with other transcription factors to inhibit Gcg expression.

In summary, the cell-type selective expression of $\mathrm{Gcg}$ is regulated through more than a dozen transcription factors that selectively bind to cis-acting elements in the $\mathrm{Gcg}$ promoter and enhancer regions, thereby either stimulating or inhibiting Gcg expression. Apart from a series of homeodomain proteins, Gcg expression is also stimulated by PKA in response to increased levels of cAMP [94,99,101,129]. Insulin stimulates intestinal Gcg expression [133] while at the same time inhibiting Gcg expression in $\alpha$-cells $[89,137,138]$. Certain effectors of the Wnt pathway further enhance Gcg expression in the intestine but not in the pancreas [94,132,143].

\section{POSTTRANSLATIONAL PROCESSING OF PREPROGLUCAGON}

The vast majority of glucagon is produced in the pancreatic $\alpha$-cells, but, under some conditions, small amounts of glucagon also have been detected in the intestinal L-cells [87], although the validity of immunoassays to distinguish different proglucagon products has been questioned, and some reports were unable to find genuine glucagon in the intestine by mass spectrometric analysis [144-146]. Glucagon also has been detected immunohistochemically in certain Gcg-positive neurons of the NTS [84-86]. The tissue-specific cleavage of proglucagon is orchestrated by the selective expression of the prohormone convertase (PC) enzymes. Prohormone convertase 1 (PC1; a.k.a. PCSK1 or PC1/3) is expressed in GCG + cells in the brain and the intestine, and cleavage of Gcg by PCSK1 results in the liberation of GLP-1, GLP-2, glicentin, oxyntomodulin, and IP2 (Figure 1) [147-150]. In contrast, PC2 (a.k.a. PCSK2) is highly expressed in the pancreas [151], and its expression in $\alpha$-cells results in cleavage of Gcg into "pancreatic type" glucagon, GRPP, MPGF, and a small intervening peptide (IP1). Studies in the porcine and human pancreas suggest that the PCSK2-liberated PGDPs are all co-secreted in equimolar concentrations from the islets [152,153]. Underlining the role of PCSK2 in liberation of glucagon via proglucagon processing, PCSK2-deficient mice are slightly hypoglycemic upon fasting, have a reduced rise in blood glucose following intraperitoneal glucose administration, display impaired processing of Gcg in the $\alpha$-cells, and develop $\alpha$-cell hyperplasia $[154,155]$. The hypoglycemia and $\alpha$-cell hyperplasia seem to directly result from glucagon deficiency because continuous intraperitoneal glucagon supplementation is sufficient to correct the hypoglycemia and the $\alpha$-cell hyperplasia of Pcsk ${ }^{-1-}$ mice [156].

While PCSK2 is the predominant prohormone convertase in $\alpha$-cells in non-pathological conditions, $\alpha$-cell PCSK1 immunoreactivity increases in rodent models of metabolic stress. $\alpha$-cell PCSK1 activity and/or expression is found in embryonic and neonatal mice, with pregnancy, and in models of prediabetes and diabetes [157-160]. In cultured 
$\alpha$-cell lines or isolated islets, high-media glucose concentrations increase PCSK1 expression and cellular GLP-1 content $[161,162]$. Islet GLP-1 production is also mediated by the cytokine IL-6, which is released in response to exercise, obesity and diabetes [163,164]. Lastly, upon streptozotocin-induced destruction of the $\beta$-cells, there is an acute increase in islet PCSK1 and Gcg expression and increased processing of proglucagon to GLP-1 [158]. Consistent with this, adenoviral overexpression of PCSK1 in $\alpha$-cells increases islet GLP-1 production and secretion, ultimately leading to enhanced glucosestimulation of insulin secretion and improved survival of the islets [165]. Further, glucagon receptor KO mice also have compensatory increases in $\alpha$-cell GLP-1, and GLP-1 receptor (GLP-1R) signaling has been reported to contribute to the preserved glucose responses after streptozotocin administration $[166,167]$. Together, these data point to a potential role of the $\alpha$-cells in compensating for increased $\beta$-cell functional demand under conditions of insulin resistance, pregnancy, and cellular stress through intra-islet GLP-1 production [168,169]. Multiple lines of evidence are accumulating that challenge current dogma and imply that pancreatic GLP-1 production also has a role, under some circumstances, in regulating insulin secretion via paracrine action [170-172]. Further work is needed to understand the pathological and physiological role for GLP-1 vs. glucagon and paracrine signaling in islet cell development. Glucagon-stimulation of insulin secretion is preserved in islets isolated from $\beta$-cell-specific GCGR $\mathrm{KO}$ mice but is attenuated upon treatment of these islets with exendin (9-39) [173]. Consistent with this, glucagon-stimulation of insulin secretion is decreased in islets isolated from $\beta$-cell-specific GLP-1R KO mice [173]. These data underscore the importance of $\alpha$-to $\beta$-cell communication and indicate that glucagon may be the dominant PGDP acting via the GLP-1R on the $\beta$-cells to stimulate insulin secretion [173].

Several forms of GLP-1 are processed from proglucagon and vary in their ability to enhance glucose-induced insulin secretion. The different forms include GLP-1 (1-37) (or 1-36amide) and two "truncated" forms, GLP-1 (7-36amide) ("amidated GLP-1) and GLP-1 (7-37) ("glycine-extended GLP-1") (Figure 1) [174]. In humans, nearly all circulating GLP-1 is one of the truncated forms, with $\sim 80 \%$ of GLP-1 immunoreactivity corresponding to GLP-1 (7-36amide) and $\sim 20 \%$ to the glycine-extended GLP-1 (7-37) [175]. The relative abundance of GLP-1 (7-36amide), GLP-1 (7-37) and GLP-1 (1-37) differs among species [176-178]. Both the longer and the truncated forms of GLP-1 are detected in extracts of rat intestine and pancreas [52], in a rat Gcgproducing cell line [179] and upon transfection of rat pituitary or rat insulinoma cells with a glucagon fusion gene [180]. While GLP-1 (736amide) and GLP-1 (7-37) are equally potent to stimulate the secretion of insulin and c-peptide [181], GLP-1 (1-37) has a much lower insulinotropic efficacy [80-82].

\section{GLP-1 DEGRADATION}

Native GLP-1 has a very short half-life, which, depending on the species, is around 1-2 min [182-184] and results from two causes: (a) the action of the enzyme dipeptidylpeptidase-4 (DPP-4) and (b) renal elimination. DPP-4 cleaves GLP-1 (7-36amide) and GLP-1 (737 ) at the N-terminal dipeptide to generate GLP-1 (9-36amide) or GLP1 (9-37), low affinity ligands for the GLP-1 receptor [185-188]. Both these intact forms as well as inactivated GLP-1 metabolites are also rapidly cleared from the circulation via the kidneys. In mice, the enzyme neprilysin additionally rapidly degrades the metabolites, making GLP-1 difficult to measure in this species [189]. While GLP-1 degradation is unaffected by kidney function, the clearance of both
GLP-1, and to a greater extent its inactive metabolites, is delayed in patients with renal insufficiency [184].

DPP-4 exists in two forms; i.e., it is a membrane-spanning cell surface protein and a circulating protein, and both forms have actions that extend beyond its proteolytic activity [190]. In the intestine, DPP-4 is highly expressed in the enterocyte brush border and in endothelial cells [191]. Consequently, as discussed in a comprehensive review [152], a large portion of intestinal GLP-1 is already degraded in the capillaries of the distal gut with an estimated $\sim 25 \%$ of active GLP- 1 reaching the liver and only $\sim 10-15 \%$ reaching the general circulation $[152,176,183,191]$. Pharmacological inhibition or genetic reduction of DPP-4 activity preserves much higher circulating levels of intact GLP-1 $[191,192]$, and this was demonstrated to potentiate the insulinotropic effect of GLP-1 in anesthetized pigs (eventually leading to the development of DPP-4 inhibitors for clinical use) [193]. When administered i.v., i.p., or s.c. in rats, GLP-1 (7-36 amide) has a half-life of $0.8-$ $4.7 \mathrm{~min}, 0.6-13.5 \mathrm{~min}$ and $4.6-7.1 \mathrm{~min}$, respectively [194].

Substantial evidence indicates that the DPP-4-generated GLP-1 metabolites (GLP-1 (9-36amide) and GLP-1 (9-37)) have no major role in regulating glucose metabolism [195-197]. However, one report of an experiment in obese humans suggested that GLP-1 (9-36amide) is a weak insulin secretagogue [198], and administration of GLP-1 (936amide) improves glucose handling without affecting insulin secretion in anesthetized pigs and in humans [199,200]. GLP-1 (9-36amide) also improves cardiac output in the post-ischemic mouse heart when administered during reperfusion, and it affects vasodilation in mesenteric arteries in mice [201]. This scenario contrasts with the apparent lack of effect of high doses of GLP-1 (9-36amide) on glucoregulation in ob/ob mice or on cognitive function in high-fat fed mice [202,203]. Indeed, there is evidence that truncated GLP-1 (9-36amide) has no effect on glucose clearance or insulin secretion in healthy humans [196]. In fact, the peptide acts as a weak GLP-1R antagonist, clearly counteracting the biological effects of GLP-1 (7-36amide) in vitro [204].

\section{REGULATION OF GLP-1 SECRETION}

\subsection{Intestinal distribution of the L-cells}

Although there is some species variation, the density of L-cells is relatively low in the proximal small bowel and increases distally along the gut, with greatest density in the ileum and colon [205-209]. In rats, pigs, and humans, the majority of L-cells are located in the epithelial layer of the distal bowel [208]. The apical surface of the L-cell faces the gut lumen, where it has direct contact with luminal nutrients (Figure 3) [208]. Consistent with this, plasma levels of GLP-1 rapidly increase in healthy humans upon direct administration of carbohydrates or lipids into the ileum [210]. While potentially being sufficient for the early rise in circulating GLP-1 upon food intake, the relative scarceness of L-cells in the proximal small intestine of both rodents and humans [208] suggests that proximal to-distal neuronal and/or humoral signals might affect the release of GLP-1 during meals [208], in particular during the early phase of GLP-1 secretion. Nonetheless, the ability of L-cells to secrete GLP-1 in direct response to luminal nutrients certainly plays an additional role, and may be the main mechanism of GLP-1 secretion. Contact of nutrients with L-cells in the distal gut likely explains the prolonged duration of elevated GLP-1 during meals, and it might also be responsible for the typically observed increased levels of GLP-1 following gastric bypass and, albeit less so, after sleeve gastrectomy surgery [211-216], because surgical repositioning of the distal gut more rapidly exposes the distal L-cells to incoming nutrients [144]. Similar effects are elicited by $\alpha$-glucosidase 

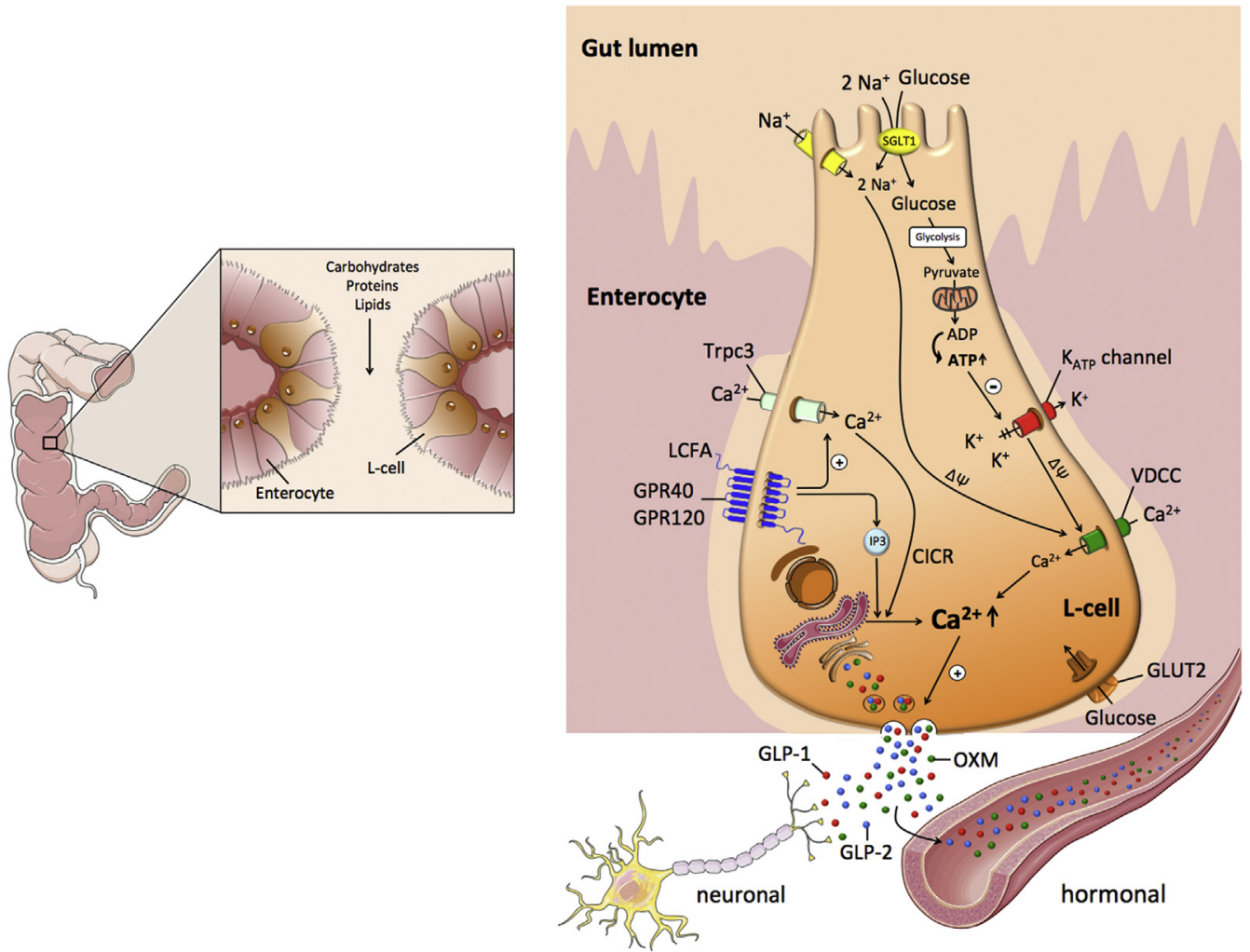

Figure 3: Schematic on the nutrient-induced stimulation of GLP-1 secretion in the L-cell. CICR: calcium-induced calcium release; LCFA: Iong-chain fatty acids, GLUT2: glucose transporter 2; GLP-1: glucagon-like peptide-1; GLP-2: glucagon-like peptide-2; OXM: oxyntomodulin; Trpc3: transient receptor potential channel 3; VDCC: voltagedependent calcium channel; SGLT1: sodium/glucose co-transporter 1. For further explanations, please see text.

inhibitors, which prevent digestion of starch and oligosaccharides, thus moving the unprocessed nutrients to more distal parts of the gut. In some studies, depending on subject characteristics and nutrient load, GLP-1 responses were augmented by acarbose or voglibose $(\alpha$ glucosidase inhibitors) [217,218]. The commonly observed increase in GLP-1 after bariatric surgery [211-216] is considered a causal factor leading to increased circulating levels of insulin after the surgery. In line with this notion, blockade of GLP-1R with exendin-9 has been demonstrated to normalize post-bariatric hyperinsulinemia and alleviate resulting hypoglycemia after RYGB [219] or gastrectomy [217]. Similarly, blockade of GLP-1R with an antagonistic monoclonal antibody demonstrated an important contribution of enhanced GLP-1 secretion to the improved insulin secretion and glucose handling after vertical sleeve gastrectomy (VSG) in preoperatively lean mice [144]. The commonly observed rise in GLP-1 following gastric bypass surgery is also consistent with the robust responses observed in humans upon direct introduction of nutrients, in amounts corresponding to the "physiological malabsorption", by distal ileal intubation [210]. Thus, increased circulating GLP-1 is invariably reported after bariatric surgery [211-216] and after surgical repositioning of the distal gut (socalled ileal interposition), both of which rapidly expose the L-cells to incoming incompletely digested nutrients [144].

Enhanced GLP-1 secretion has been suggested to be a major mechanism underlying enhanced insulin secretion after bariatric surgery because blockade of GLP-1 action by treatment with the pharmacological antagonist exendin-9 decreased insulin secretion and ameliorated post-surgical glucose handling in both mice and humans [144]. Nonetheless, a primary role of GLP-1 in post-surgical improvement of metabolism is controversial. Studies in GLP-1R KO mice documented that obese mice exhibit comparable weight loss and improved glucose metabolism to wildtype controls following VSG [220]. Failure of GLP-1R $\mathrm{KO}$ to attenuate the beneficial effects of bariatric surgery on body weight may be related to the observation that the body weight decrease in mice after RYGB may reflect enhanced energy expenditure, whereas in humans and in rats a reduction in food intake is more important [221]. However, sleeve gastrectomy differs markedly from gastric bypass with faster systemic appearance of ingested glucose and higher secretion of insulin, GLP-1, PYY, CCK, and ghrelin after RGYB [222]. Perhaps analogously, rodent experiments have found that GLP-1R signaling is not required for the weight-reducing effect of Roux-en-Y Gastric Bypass (RYGB) [223]. Furthermore, GLP-1 inhibition by exendin (9-39) or administration of a DPP-4 inhibitor does not affect food intake in humans following RYGB [224]. However, as demonstrated in that same report, concomitant administration of exendin9-39 and a DPP-4 inhibitor increased food intake by $\sim 20 \%$, suggesting that GLP-1, when potentially acting in concert with other gut hormones, might have an important role in the metabolic benefits achieved by bariatric surgery [224]. Consistent with this, treatment of RYGB patients with exendin (9-39) increases the fMRI response to images of food in the caudate nucleus, and in the insula in the human brain in response to consuming palatable food [225].

The improved glucose metabolism is mainly due to three closely interacting factors: 1) rapid absorption of glucose from the intestine giving rise to high post-prandial glucose responses, 2) a consequent exaggerated secretion of GLP- 1 that act on the $\beta$-cells, and 3) radically improved hepatic and subsequently peripheral insulin sensitivity [226]. 
Like pancreatic $\alpha$-cells, the intestinal L-cells secrete PGDPs simultaneously and in equimolar concentrations. An exception is glicentin and its cleavage product oxyntomodulin, the concentrations of which need to be combined to match the amount of secreted GLP-1 [41,53]. Mealinduced GLP-1 secretion has been demonstrated in numerous species including mice [227], rats [228-232], dogs [233-235] and humans [236-239]. An important consideration when analyzing meal-induced GLP-1 secretion is whether GLP-1 was measured in systemic or portal vein blood, whether the samples were taken in relation to oral meals or rather to intragastric infusion of liquid (or semiliquid) meals, and whether total or active GLP-1 was measured. As comprehensively discussed previously [240], an estimate of up to $75 \%$ of active GLP-1 is already degraded in the gut, and, from the amount of GLP-1 reaching the liver, another $50 \%$ is degraded before reaching the systemic circulation [183,241]. Only $10-15 \%$ of active GLP-1 is believed to reach the pancreas via the circulation $[185,191,241]$. As demonstrated in anesthetized pigs, blood concentrations of total and active GLP-1 progressively decrease with increasing distance from the site of secretion [241]. Levels of total and active GLP-1 are thus highest in the portal vein and lowest in the peripheral venous system [241]. In anesthetized pigs, baseline levels of total GLP-1 in the portal vein are in the range of $\sim 30 \mathrm{pmol} / \mathrm{l}$ and increase up to $150 \mathrm{pmol} / \mathrm{l}$ upon treatment with neuromedin C, which is a known stimulator of GLP-1 secretion [241]. Baseline portal vein levels of active GLP-1 are $\sim 10 \mathrm{pmol} / \mathrm{l}$ and increase up to $\sim 40 \mathrm{pmol} / \mathrm{l}$ upon treatment with neuromedin C [241].

Systemic plasma levels of total C-terminally amidated GLP-1 correlate with plasma insulin and thus are low during fasting and increased in response to an oral meal [236]. In humans, fasting systemic plasma concentrations of "total" GLP-1 (including metabolites generated by DPP-4-mediated degradation) are typically in the range of $5-10 \mathrm{pmol} /$ $\mathrm{L}$ and can increase up to $40 \mathrm{pmol} / \mathrm{L}$ in response to a meal [236]. Plasma systemic concentrations of "intact", biologically active GLP-1 are much lower (fasting: $<2 \mathrm{pmol} / \mathrm{l}$, peak post-nutrient concentrations $5-10 \mathrm{pmol} / \mathrm{l})[240,242]$. The amount of GLP-1 secretion is affected by the size and composition of a meal [237]. In healthy human volunteers, a $520 \mathrm{kcal}$ oral meal induced a greater systemic increase of plasma systemic total and active GLP-1 than a $260 \mathrm{kcal}$ meal [237]. GLP-1 levels are detectable during fasting, indicating that GLP-1 is tonically secreted into the general circulation in basal conditions [243]. In rats however, there is also evidence for a "preprandial" cephalicphase GLP-1 reflex [244,245], and the muscarinic cholinergic antagonist atropine reduces the GLP-1 response to an oral glucose load in humans [246].

Nutrients stimulating GLP-1 secretion include the metabolizable monosaccharides that include glucose, fructose, and galactose $[239,247-250]$ as well as non-metabolizable monosaccharides such as methyl- $\alpha$-glucopyranoside [247], long-chain fatty acids [232,251253], proteins [228,250,254-257], and certain amino acids [258260]. In healthy humans, ingestion of carbohydrates or proteins elicits a rapid increase in circulating GLP-1 with a peak 30-60 min following nutrient intake, whereas ingestion of lipids elicits a somewhat later but more prolonged (>120 min) increase [238,239]. In rats, portal vein levels of total GLP-1 peak around 15 min after intragastric infusion of a liquid meal and return to baseline levels after 90$120 \mathrm{~min}$ [231]. In rats eating a spontaneous chow meal, increased portal vein but not systemic levels of active GLP-1 are detectable [261]. There is controversy as to whether glucose-induced GLP-1 secretion is disturbed in patients with type-2 diabetes. A study in $\sim 1,500$ Danish subjects suggested that the GLP-1 response to oral glucose is reduced in patients with prediabetes or type-2 diabetes [262]. In contrast, a meta-analysis of 22 clinical studies revealed no difference in glucosestimulated GLP-1 secretion between patients with type-2 diabetes and non-diabetic controls [263,264].

\subsection{GLP-1 secretion in response to monosaccharides and other carbohydrates}

The cellular mechanisms underlying glucose-stimulation of GLP-1 secretion from L-cells are, at least in part, similar to the stimulation of insulin secretion in the islets. In enteroendocrine GLUTag cells, glucose, and fructose dose-dependently increase GLP-1 secretion through closure of ATP-sensitive $\mathrm{K}_{\text {ATP }}$ channels and subsequent membrane depolarization (Figure 3) [247,249,265]. Glucose-induced membrane depolarization entails opening of voltage-dependent $\mathrm{Ca}^{2+}$ (VDC) channels, and the resulting $\mathrm{Ca}^{2+}$ influx then triggers vesicular exocytosis and secretion of GLP-1 into the circulation (Figure 3) [266]. Underlining the role of the $\mathrm{K}_{\text {ATP }}$ channels in mediating this process, glucose-stimulated $\mathrm{Ca}^{2+}$ entry and GLP-1 secretion are mimicked upon treatment of GLUTag cells with the KATP channel inhibitor tolbutamide [265]. While the importance of $\mathrm{K}_{\text {ATP }}$ channel activity in mediating GLP-1 release has been confirmed in vitro, its relevance for GLP-1 secretion in vivo is less clear. While sulphonylureas potently promote insulin secretion in type-2 diabetic patients via inhibition of $\mathrm{K}_{\text {ATP }}$ channel activity [267-270], there is no clear evidence that sulphonylureas affect GLP-1 secretion in humans (as reviewed in [266]). However, the Kir6.2/SUR1 channel complex of the $\mathrm{K}_{\text {ATP }}$ channel is present in human L-cells [271] and $\mathrm{K}_{\text {ATP }}$ channel subunits and glucokinase are highly expressed in murine L-cell populations [266]. In summary, while L-cell depolarization is crucial for GLP-1 secretion, the role of the Kir6.2/SUR1 channel complex of the KATP channels for mediating this process in vivo warrants clarification.

Monosaccharides demonstrated to stimulate GLP-1 secretion include glucose, galactose, and fructose [239,247]. Low concentrations of glucose or methyl- $\alpha$-glucopyranoside stimulate L-cell electrical activity and promote GLP-1 secretion via sodium-glucose cotransporter (SGLT1)-dependent induction of small inward currents (Figure 3) [247]. Preventing luminal glucose absorption by blockade of SGLT1 reduces GLP-1 secretion in the isolated perfused canine ileum [248] and in the rat ileum [178] and impairs glucose-stimulation of GLP-1 release in GLUTag cells [247]. Notably, the glucose transporter-2 (GLUT2) has been implicated in glucose-stimulated GLP-1 secretion, as demonstrated by an impaired GLP-1 response to oral glucose in mice deficient for GLUT2, and this is accompanied by reduced glucosestimulated insulin secretion and impaired glucose tolerance [272]. However, pharmacological inhibition of active, sodium-coupled glucose transport impaired glucose-stimulated GLP-1 secretion in vitro, whereas inhibition of facilitative GLUT-mediated glucose transport was without effect [273]. In summary, glucose uptake into the L-cells seems to be mediated via both, GLUT2 and SGLT1 (Figure 3) [274-276], with the electrogenic SGLT1 mediated uptake being of particular importance for stimulus secretion coupling. Downstream of glucose-mediated membrane depolarization, vesicular exocytosis of GLP-1 is orchestrated in a $\mathrm{Ca}^{2+}$ dependent manner involving a cellular machinery like that in $\beta$-cells $[277,278]$. Fructose stimulation of GLP-1 secretion has been demonstrated in rats, mice, humans, and GLUTag cells [249,279]. When given orally, fructose is a far less potent GLP-1 secretagogue relative to an isocaloric load of glucose [249]. Similar findings are reported in humans upon intragastric infusion of glucose and fructose at doses that are matched for sweetness [279].

A possible role of intestinal sweet-taste receptors in glucosestimulated GLP-1 secretion remains uncertain. In isolated murine 
and human L-cell cultures, glucose, and the artificial sweetener sucralose, each stimulates GLP-1 secretion with diminished glucose stimulation of GLP-1 secretion in mice lacking $\alpha$-gustducin, an integral sweet-taste receptor element [280]. In contrast, there is no effect of sucralose on GLP-1 secretion in primary L-cell cultures in other reports [281,282], and infusion of artificial sweeteners does not affect glucose-stimulated GLP-1 secretion in healthy human volunteers [283,284].

\subsection{GLP-1 secretion in response to dietary lipids}

In addition to sensing carbohydrates and glucose, L-cells, like other enteroendocrine cells, sense dietary lipids and protein (see next section) through specific cell-surface receptors, which bind metabolites of the dietary lipids and proteins [285] (Figure 4). This occurs mainly at the basolateral side of the L-cell, i.e. after absorption of the metabolites [285]. In the case of dietary triglycerides, the L-cell responds not only to the free fatty acids but also to the other major metabolite product, 2monoacyl glycerol (2-MAG), which appears to act in synergy [286]. Cell lines commonly used to study mechanisms of GLP-1 secretion are GLUTag, STC-1 and human colorectal adenocarcinoma-derived NClH716 cells. These cell lines are far from optimal models, as they, for example, differ in their secretory repertoire; some secreting peptides that are not classical L-cell products, such as GIP, glucagon, and somatostatin, and GLUTag cells lack PYY [287].

Fatty acid induction of GLP-1 secretion has been demonstrated in vitro using murine enteroendocrine STC-1 cells [251] and human intestinal $\mathrm{NCl}-\mathrm{H} 716$ cells $[251,253]$ and in vivo by direct administration of lipids into the duodenum $[288,289]$ or ileum [210]. Dose-dependent fatty acid-induced GLP-1 secretion is evident with $\alpha$-linolenic acid (C18:3), docosahexanoic acid (C22:6), and palmitoleic acid (C16:1), oleic acid (C18:1), stearic acid (C18:0), and octanoic acid (C8:0) [251] are less effective. In humans, unsaturated are more effective than saturated fatty acids [290,291].

Induction of GLP-1 secretion by FFA is highly dependent on the cytosolic $\mathrm{Ca}^{2+}$ concentration. Treatment of STC-1, GLUTag, or NCI$\mathrm{H} 716$ cells with long-chain fatty acids potently increases intracellular $\mathrm{Ca}^{2+}[253,292]$. The FFA-induced rise in intracellular $\mathrm{Ca}^{2+}$ is substantially reduced when cells are cultured in $\mathrm{a} \mathrm{Ca}^{2+}$ free medium, and is abolished upon treatment of cells with the $\mathrm{Ca}^{2+}$ channel inhibitor nicardipine [292] or when using BSA (which binds fatty acids) [251] Collectively, these data suggest that FFAs increase intracellular $\mathrm{Ca}^{2+}$ by stimulating the influx of $\mathrm{Ca}^{2+}$ via the cell-surface (most likely Ltype) $\mathrm{Ca}^{2+}$ channels [292]. Treatment of NCl-H716 or STC-1 cells with the $\mathrm{Ca}^{2+}$ ionophore ionomycin or with phorbol myristate acetate (PMA) increases cytosolic $\mathrm{Ca}^{2+}$ levels and stimulates GLP-1 secretion in a dose-dependent manner [251,253]. The ionomycin-induced increase in $\mathrm{Ca}^{2+}$ influx and GLP-1 secretion is completely abolished upon treatment of cells with the $\mathrm{Ca}^{2+}$ chelator EGTA [251]. In summary, compelling evidence indicates that FFA increase GLP-1 secretion by stimulating extracellular $\mathrm{Ca}^{2+}$ influx via cell-surface $\mathrm{Ca}^{2+}$ channels. Receptors implicated in FFA regulation of GLP-1 secretion include GPR120 (FFAR4) and GPR40 (FFAR1), both of which are activated by long-chain fatty acids. GPR120 was previously reported to be colocalized with GLP-1 in colonic enteroendocrine cells [251]. This could point to GPR120 being involved in the stimulation of GLP-1 by long chain FFAs. However, The GLP-1 response to oleic acid is unaltered in GPR120-deficient mice, and synthetic GPR120 agonists do not stimulate GLP-1 from primary cell cultures [286]. GPR40, the other long chain FFA receptor, is also highly expressed and the most enriched GPCR in L-cells [266,293]. In contrast to GPR120, the GLP-1 response to dietary fat is strongly reduced in GPR40 KO mice as compared to littermates [286]. Importantly, synthetic GPR40 agonists

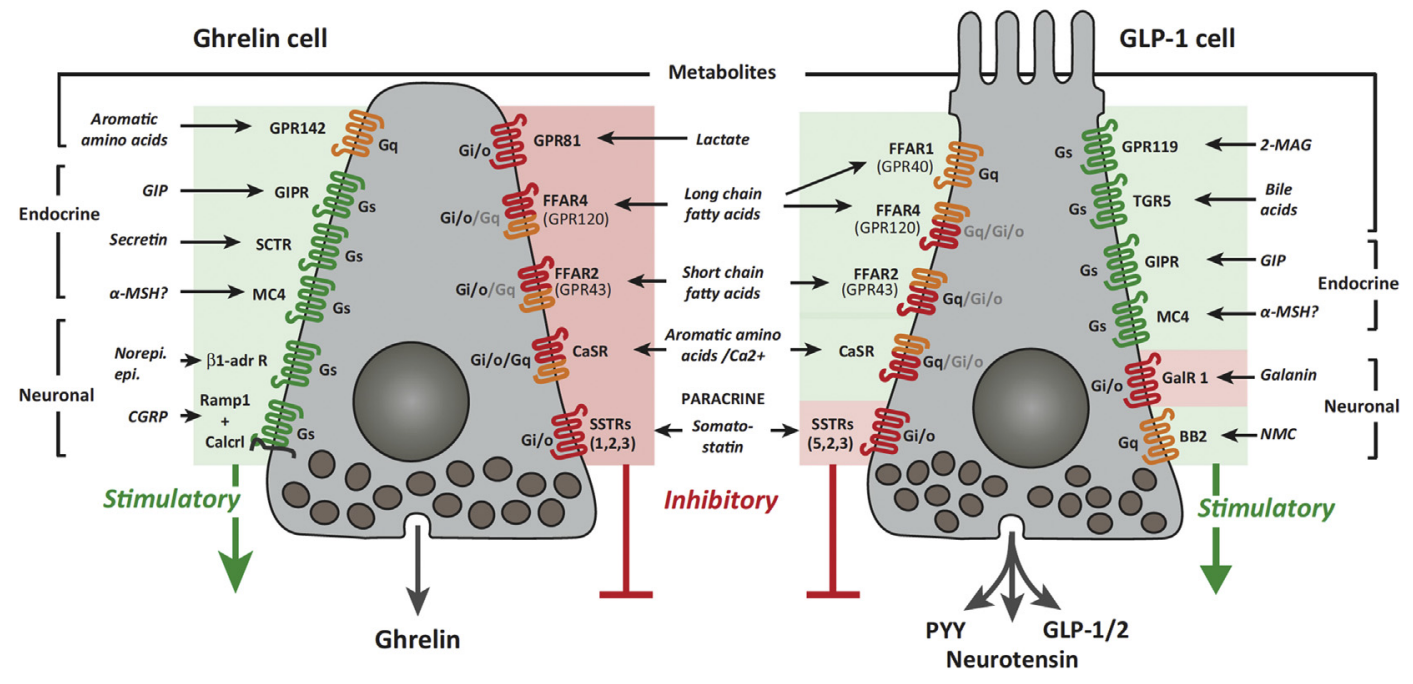

Figure 4: Schematic on the GPCR Repertoire Involved in Control of Hormone Secretion from Gastric cells expressing either ghrelin or GLP-1. Green or red color background color indicates stimulation (green) or inhibition (red) of hormone secretion upon receptor activation. The colors of the receptors represent $G$ protein signaling potential via $\mathrm{G} / \mathrm{s}$ (green), $\mathrm{G} / \mathrm{q}$ (orange) or $\mathrm{G} / \mathrm{i} / \mathrm{o}$ (red). The type of $\mathrm{G}$ protein responsible for the effect on hormone secretion has not been determined for all the receptors; for those where it has not been described, we have indicated the most likely coupling, based on data from other cell studies. 2-MAG, 2- monoacyl glycerol; BB2, bombesin receptor 2; Calcrl, calcitonin receptor-like receptor; CaSR, calcium sensing receptor; CGRP, calcitonin gene-related peptide; FACS, fluorescence-activated cell sorting; FFAR, free fatty acid receptor; GalR1, galanin receptor 1; GIP, glucose-dependent insulinotropic peptide; GIPR, GIP receptor; GPR, G protein receptor; MC\$, melanocortin 4; MSH, melanocyte-stimulating hormone; NMC, neuromedin C; PYY, peptide YY; Ramp1, receptor activity modifying protein 1; SCTR, secretin receptor T; SSTR, somatostatin receptor; TGR5, bile acid receptor. Figure provided by the courtesy of Prof. T. W. Schwartz. 
are efficacious GLP-1 secretagogues both in vitro in primary cultures, in perfused intestines and in vivo when studied in mice [252,294,295]. Treatment of mice with GPR40 agonists [296,297] has been reported to decrease body weight and food intake in DIO mice, effects notably absent in mice deficient for either GPR40 or GLP-1R [296].

Accordingly, long chain fatty acids stimulate GLP-1 secretion mainly through GPR40. However, the other main metabolite from dietary triglycerides, 2-MAG, is also a very powerful GLP-1 secretagogue acting through GPR119 [298,299]. Importantly, the GLP-1 response to dietary fat is vastly reduced not only in GPR40 K0 animals but also in GPR119 $\mathrm{KO}$ mice, and agonists for the Gq-coupled GPR40 act in synergy with the Gs-coupled GPR119 to robustly stimulate GLP-1 [286,300]. A receptor-independent stimulation of GLP-1 release from GLUTag cells has been reported for oleic acid, which stimulates GLP-1 release by uncoupling oxidative phosphorylation and, hence, indirectly stimulating glycolysis with the resulting activation of the mechanism alluded to above [301].

\subsection{GLP-1 secretion by proteins/amino acids}

Protein and amino acid stimulation of GLP-1 secretion has been demonstrated in murine primary colonic L-cell cultures [260,302], in GLUTag cells [256,259], in human NCl-H716 cells [251,303] and in the isolated perfused rat ileum or colon $[250,304]$ as well as in vivo in mice [305], rats [256,306], and humans [307,308]. In healthy human volunteers, a diet with $30 \% \mathrm{kcal}$ from protein $(40 \%$ carbohydrates, $30 \%$ fat) causes greater GLP-1 secretion than a diet with $10 \% \mathrm{kcal}$ protein (60\% carbohydrates, $30 \%$ fat) [308]. Individual amino acids stimulating GLP-1 secretion include glutamine, asparagine, phenylalanine, and glycine, with glutamine and glycine being the most potent [258260]. When orally administered, glutamine also increases circulating GLP-1 and insulin in lean, obese and type-2 diabetic individuals [307]. In human $\mathrm{NCl}-\mathrm{H} 716$ cells, stimulation of GLP-1 secretion was also demonstrated for leucine, isoleucine, valine, skimmed milk, casein, and whey [303]. L-Arginine, a potent insulin secretagogue [309], also stimulates GLP-1 release from isolated rat intestine, and, when given orally, augments GLP-1 and insulin levels and improves glucose tolerance in mice, effects that are absent in GLP-1R KO mice [305]. Meat hydrolysate stimulates GLP-1 secretion from $\mathrm{NCl}-\mathrm{H} 716$ cells, an effect that is not related to changes in proglucagon expression [253]. This stimulatory effect can be blocked by pretreatment with the p38 inhibitor SB203580, the PI3 kinase inhibitor wortmannin or the MEK1/2 inhibitor U0126 [310]. The corn protein zein stimulates GLP-1 secretion in GLUTag cells and in the small intestine of anesthetized rats [256], and it stimulates GLP-1 secretion when administered either orally [228] or directly into the ileum [257]. Intraluminal administration of peptones stimulate GLP-1 secretion in the isolated perfused rat ileum [250] but not upon ileal perfusion in healthy human volunteers [210]. Pectin stimulates GLP-1 secretion in the isolated perfused rat colon [304] but not in the isolated rat ileum [250]. The low molecular fraction of wheat protein hydrolysate (LWP) increases GLP-1 secretion in both GLUTag cells and when directly administered in rats [306]. In rats, the LWP-induced GLP-1 secretion further improves glucose tolerance and enhances insulin secretion, an effect that is blocked by pre-administration of the GLP-1R antagonist exendin (9-39) [306]. Protein-stimulation of GLP-1 secretion has also been demonstrated in humans, with similar GLP-1 responses upon uptake of whey, casein, gluten or cod protein [311-313]. Protein-induction of GLP-1 secretion seems to be dose-dependent, as demonstrated by uptake of isocaloric diets comprising $14 \%, 25 \%$, or $50 \%$ of energy coming from proteins [314].
The molecular mechanisms underlying protein stimulation of GLP-1 secretion include activation of $\mathrm{Ca}^{2+l}$ calmodulin-dependent kinase II [306]. Substantial evidence supports that peptide-mediated GLP-1 secretion is $\mathrm{a} \mathrm{Ca}^{2+}$ sensitive process and involves L-cell signaling via the $\mathrm{Ca}^{2+}$ sensing receptor (CaSR) and the peptide transporter 1 (PEPT1) [302]. Consistent with this, glycine-sarcosine (Gly-Sar) stimulation of GLP-1 secretion from purified murine L-cell cultures is blocked in the absence of extracellular $\mathrm{Ca}^{2+}$ and is inhibited upon treatment with the L-type $\mathrm{Ca}^{2+}$-channel blocker nifedipine [302]. Oligopeptide stimulation of GLP-1 release is impaired upon treatment of L-cell cultures with a CaSR antagonist and is ameliorated in mice deficient for the peptide transporter 1 (PEPT1) [302]. Aromatic amino acids such as phenylalanine, however, also interact with GPR142 [315].

\subsection{GLP-1 secretion in response to endocrine factors}

\subsubsection{Endocrine regulation of intestinal GLP-1 secretion}

The intestinal distribution of L-cells, with high abundance in the distal gut and low abundance in the proximal gut, argues for the existence of a proximal-to-distal coordinating loop in which neuronal and/or endocrine factors arising in the upper intestine affect L-cell GLP-1 secretion in the distal region. While such a proximal-distal loop might indeed exist, it cannot be ruled out that while fewer in number, L-cells in the upper intestine are sufficient for the rapid induction of GLP-1 secretion following nutrient intake [316-318]. Nonetheless, the presumed loop (if it exists) would likely be important for the early postprandial phase at a time when the L-cells of the distal gut are not yet in direct contact with luminal nutrients. Supporting such neuronal/ endocrine regulation of GLP-1 secretion, the L-cells are in close proximity to both enteric neurons and the intestinal microvasculature $[191,319]$. Possible neuroendocrine regulation of GLP-1 secretion is supported by studies in rodents in which nutrient flow to the distal intestine is prevented, precluding direct L-cell contact in this part of the intestine to luminal nutrients, [289,320]. Administration of glucose or fat directly into the duodenum of such rodents rapidly stimulates L-cell GLP-1 secretion, with a magnitude comparable to that occurring when nutrients are directly placed into the ileum [289,320]. Because the Lcells co-secrete the PGDPs, factors stimulating GLP-2 or oxyntomodulin are also natural secretagogues of GLP-1. Neuronal/endocrine factors affecting the intestinal release of PGDPs in some species include GIP, acetylcholine, gastrin-releasing peptide (GRP), insulin, somatostatin, and ghrelin [320-322].

In rodents, a biphasic secretion of GLP-1 has been observed; this suggests a rapid phase of GLP-1 secretion caused by direct stimulation of L-cells in the upper Gl tract and a second phase potentially caused by signals from the upper gut reaching the lower small and perhaps even the large intestine [323,324]. GIP and the intramural intestinal autonomous nervous system have been suggested as signaling pathways [323]. Several lines of evidence support a role for GIP in GLP1 secretion. GIP stimulation of GLP-1 secretion, however, seems to be highly species-specific. Levels of GIP expression (in K-cells) are greatest in the proximal gut, and circulating levels of GIP rapidly increase upon food intake $[236,325,326]$ or when nutrients are placed directly into the duodenum [289,323,327]. Secretion of preproglucagon-derived peptides is stimulated in rats and in primary rat L-cell cultures upon treatment with GIP [289,323,327]. In rats, induction of gut glucagon-like immunoreactivity induced by either lipids or by physiological concentrations of GIP can be blocked by subdiaphragmatic vagotomy, suggesting that in rats GIP regulation of 
GLP-1 secretion requires either afferent or efferent signal transmission via the vagus [327]. The importance of such a proximal-to-distal GIPGLP-1 axis for human physiology is, however, questionable. In healthy and type-2 diabetic humans, it has been consistently demonstrated, that GIP, even in rather high (supraphysiological) doses, does not lead to GLP-1 secretion [328-330]. In humans, GLP-1 secretion in response to duodenal glucose delivery does not become robustly stimulated until the delivery rate overcomes the absorptive capacity of the duodenum, i.e. until such time as non-absorbed glucose reaches the jejunum and beyond. In contrast, GIP secretion is stimulated by very low gastric glucose delivery rates, consistent with the higher abundance of GIP-producing K-cells compared to GLP-1-producing Lcells in the proximal intestine [331]. Direct infusion of glucose into the duodenum at a rate that ensured $\sim$ total absorption close to the infusion site ( $2 \mathrm{kcal} / \mathrm{min})$ triggered robust GIP, but not GLP-1 secretion, whereas both hormones were released when glucose was directly delivered into the ileum [332]. Another gut peptide that has been implicated in a proximal-to-distal loop to stimulate GLP-1 secretion is CCK [333]. However, the concentrations of both GIP and CCK needed to stimulate GLP-1 secretion are not normally reached under physiological conditions [334].

Neurotransmitters expressed in vagal and enteric neurons, including acetylcholine and GRP, increase GLP-1 secretion, supporting the possibility of a proximal-distal neuroendocrine loop [335] without the need of supraphysiological plasma concentrations of proximallysecreted gut peptides. Receptors for acetylcholine, including the muscarinic receptors $\mathrm{M} 1, \mathrm{M} 2$, and $\mathrm{M} 3$, are expressed in rat L-cells [319] and human NCl-H716 cells [336]. Treatment of rats with the nonspecific muscarinic receptor antagonist atropine or with the M1 selective antagonist pirenzipine, but not treatment with M2 or M3selective antagonists blunts lipid-induced GLP-1 secretion [319,335]. In human $\mathrm{NCl}-\mathrm{H} 716$ cells, GLP-1 secretion is stimulated by bethanechol, a nonselective muscarinic agonist, while pretreatment with pirenzipine or the M2 antagonist gallamine inhibits bethanecholinduction of GLP-1 secretion [336]. Acetylcholine also stimulates GLP-1 secretion in the perfused porcine ileum, and this effect can be blocked by co-infusion of atropine [337]. Albeit with notable physiological challenges (atropine powerfully inhibits Gl motility), infusion of atropine also blunts nutrient induced GLP-1 secretion in healthy human volunteers [246]. Together, these data suggest that M1 and M2 muscarinic receptors are implicated in human L-cell GLP-1 secretion. Interestingly, in rat ileum preparations, administration of atropine was not able to block GIP-induced GLP-1 secretion [335]. In the isolated perfused porcine ileum, GLP-1 secretion is inhibited by electrical nerve stimulation or by administration of norepinephrine, effects that can be blocked by co-infusion of the nonselective $\alpha$-adrenergic receptor antagonist phentolamine [337]. While norepinephrine seemingly inhibits GLP-1 secretion via its action on $\alpha$-adrenergic receptors, its secretion is stimulated by isoproterenol, and this effect can be blocked by co-infusion of the $\beta$-adrenergic receptor antagonist propanolol [337]. These data collectively suggest that intestinal GLP-1 secretion is stimulated by cholinergic and $\beta$-adrenergic receptor signaling and inhibited by activation of $\alpha$-adrenergic receptors.

GRP is produced and released by GRPergic neurons of the enteric nervous system [338]. In rats, infusion of GRP stimulates the secretion of GLP-1 while administration of the GRP antagonist BW10 blocks GLP-1 secretion when fat is directly administered into the duodenum [339]. GRP stimulation of GLP-1 secretion has also been demonstrated for rat L-cell cultures and in preparations of rat ileum [323,335]. Notably, GRP regulation of glucose handling is not fully dependent on GLP-1 signaling, because GRP also directly stimulates insulin secretion in the isolated perfused dog pancreas [340] and further delays gastric emptying [341,342]. Nonetheless, mice deficient for GRP have impaired glucose tolerance, reduced first-phase insulin secretion and impaired GLP-1 secretion in response to an oral glucose challenge [343].

In summary, there are several mechanisms that may contribute to the rapid increase in GLP-1 secretion following nutrient intake. Nutrientinduced GLP-1 secretion can occur from L-cells located in the proximal small intestine with induction of GLP-1 secretion as early as digested nutrients leave the pylorus. The glucose concentration after a meal may exceed the absorptive capacity in the proximal intestine so that the ingested glucose rapidly reaches the more distally located Lcells. Neuroendocrine reflexes may also trigger GLP-1 secretion in addition to direct nutrient-induced stimulation of L-cell GLP-1 secretion [344]. Current data suggest that when chyme enters the duodenum it triggers GIP release in the proximal gut. The local increase in GIP stimulates vagal afferent transmission followed by activation of vagal efferents and enteric neurons that release acetylcholine and/or GRP to stimulate GLP-1 release from the distal gut. When the nutrients subsequently reach the distal gut, direct contact with the L-cells then triggers additional GLP-1 secretion into the circulation (as also reviewed in [320]).

Other factors influencing GLP-1 secretion include activation of the olfactory receptor OR51E1 using nonanoic acid, which stimulates secretion of GLP-1 and PYY in human and rodent enteroendocrine Lcells [345]. More recently, ghrelin was identified to stimulate GLP-1 secretion in murine and human L-cell cultures [322]. In mice, peripheral administration of ghrelin further enhances glucose-stimulated GLP-1 secretion and improves glucose tolerance, an effect that is blocked by pre-administration of the ghrelin receptor antagonist D-Lys GHRP6 and that is absent in GLP-1R KO mice [322].

\subsubsection{Endocrine regulation of central GLP-1 secretion}

As discussed above, in addition to enteroendocrine L-cells, GLP-1 is also produced in a discrete set of non-TH-positive neurons in the caudal portions of the NTS [86,148,346-348], and these hindbrain GCG + positive neurons are the primary source of endogenous brain GLP-1 [90]. Either peripheral administration of leptin [349] or gastric balloon distention [350] acutely activates GLP-1-producing neurons in the NTS, as assessed by cFos immunoreactivity. Direct electrical stimulation of the NTS evokes glutamatergic excitatory post-synaptic currents (EPSCs) in GCG + positive neurons [351]. Generation of mice that express eYFP under control of the Gcg promoter has enabled the isolation and characterization of NTS GCG + neurons in ex vivo tissue slices [351]. Electrophysiological whole-cell voltage- and current-clamp recordings in horizontal or coronal brainstem slices has revealed a rapid leptin-induced depolarization of these NTS GCG + neurons, thus confirming the ability of leptin to directly stimulate central GLP-1 secretion [351]. Of note, the hindbrain GCG + neurons lack the GLP-1 receptor such that they cannot be directly activated by peripherally-derived GLP-1 [351]. In addition, neither electrophysiological administration of PYY, melanotan II, nor ghrelin stimulates these neurons in isolated NTS brain slices [351]. In contrast, leptin [351], CCK, and epinephrine [352] stimulate Gcg neurons. In the NTS, neurons expressing GLP-1 also express the leptin receptor [351,353]. Electrical stimulation of the solitary tract indicates that PPG neurons in the NTS are second-order neurons that receive direct input from vagal afferents. Thus, peripheral endocrine signals, such leptin or GLP-1, can via activation of vagal afferents trigger central activation of PPG neurons in the NTS [351].

CCK-induced firing of the NTS GCG + neurons can be blocked by treatment with the glutamate receptor antagonist DNQX or by inhibition 
of a1-adrenergic signaling [352]. Consistent with these findings, peripherally administered CCK induces cFos immunoreactivity in GLP1-producing neurons of the hindbrain vagal complex of the NTS [354], and surgical vagal deafferentation reduces CCK-induced NTS neuronal cFos activation by approximately $50 \%$ [355]. Thus, these neurons are able to sense and respond to a variety of peripheral signals that help to regulate both short and long-term energy balance. Indeed, similar to chronic blockade of CNS GLP-1R, viral knockdown of GLP- expressing neurons in rats increases body mass, and specifically body adiposity [356]. In a recent report, chemogenetic stimulation of Gcg neurons reduced food intake without conditioning avoidance, and this occurred when the animals were fed or fasted or were fed chow or HFD [357]. On the other hand, acute chemogenetic inhibition of these neurons did not increase ad lib feeding but did increase refeeding after a fast and blocked stress-induced hypophagia [90]. In summary, the glutamatergic GLP-1-producing neurons in the NTS are activated by multiple peripheral signals and regulate many aspects of feeding behavior. This CNS GLP-1 system does not seem to be activated by peripherallysecreted (endogenous) GLP-1 and therefore may be distinct from the peripheral GLP-1 system.

\section{THE GLUCAGON RECEPTOR FAMILY}

GLP-1, GLP-2, glucagon, GIP, secretin, and growth hormone-releasing hormone (GHRH) belong to a group of structurally related peptides that promote their biological action via binding to structurally similar $\mathrm{G}$ protein-coupled receptors (GPCRs) of the class B family [358,359]. All members of this family are seven transmembrane $\mathrm{G}_{\mathrm{S}}$-coupled receptors that increase levels of CAMP through activation of adenylate cyclase [359]. Each receptor of this family is concisely named based on its single and unique endogenous ligand (GLP-1R, GLP-2R, GCGR, GIPR, SCTR, and GHRHR). Under physiological conditions, most studies report no meaningful cross-reactivity among the peptide ligands and the receptors of this family $[359,360]$.

Cloning of rat and human pancreatic GLP-1R cDNA documented that ligand-induced activation of a single unique GLP-1R increases intracellular levels of CAMP and also that GLP-1, but not glucagon, GIP, VIP, or secretin, activates GLP-1R [360-364]. In the pancreas, glucagon has physiologically relevant cross-reactivity with GLP-1R, with an $\mathrm{EC}_{50}$ of $36.4 \pm 0.22 \mathrm{nM}$, but there is no affinity of GLP-1 to the glucagon receptor [365]. The interaction of glucagon with GLP-1R is important for insulin secretion in the $\beta$-cells [365]. Cloning of the human pancreatic GLP-1R cDNA was also used to demonstrate comparable binding affinity of exendin-4 and exendin (9-39) to human GLP-1R [363], and consolidated the work of Jean-Pierre Raufman and John Eng that identified exendin-4 as a GLP-1 paralog [366] and exendin $(9-39)$ as a GLP-1R antagonist [367].

\subsection{Tissue distribution of GLP-1R}

The presence of GLP-1R was first demonstrated in rat insulinoma RINm5F [368], RIN5AH [369], and RIN1046-38 cells [82] using cAMP accumulation assays and radioligand binding. Subsequent studies then confirmed the presence of GLP-1R in these and other insulinoma cell lines [369-376] as well as in somatostatin-secreting cells [370,375] and in islets isolated from rats [377] and humans [378].

Expression of GLP-1R was also demonstrated in the rat lung [379] as well as in the human brain, kidney, stomach and heart, with no expression of GLP-1R in liver, skeletal muscle or adipose tissue $[380,381]$ Although early efforts [380,381] failed to consistently detect the GLP-1 receptor in adipose tissue, more recent studies have unequivocally identified the GLP-1R expressed in adipocytes. These recent studies include the finding of the receptor in differentiating mouse 3T3-L1 pre-adipocytes [382-384], adipocytes formed from human bone marrow-derived mesenchymal stem cells [385], human epicardial fat [386], and in human visceral white adipose tissue [387]. Nonetheless, GLP-1 and liraglutide induce adipocyte formation in vitro and in vivo, and adipogenesis is decreased in preadipocytes lacking GLP-1R [384]. Autoradiography studies identified binding of [125I]GLP1 and [125I][Tyr39] exendin-4 in the rat lateral septum, subfornical regions, thalamus, hypothalamus, interpenduncular nucleus, posterodorsal tegmental nucleus, area postrema, inferior olive, and NTS [388]. Similar findings on the central distribution of GLP-1R mRNA in rats have been reported using S35-UTP-labeled probes complementary to the PPG and GLP-1R mRNA [347]. In non-human primates, the highest GLP-1 immunoreactivity has been demonstrated in various nuclei of the hypothalamus, the area postrema, the NTS, and the dorsal motor nucleus of the vagus [389]. In the human brain, expression of GLP-1R mRNA has been reported in the cerebral cortex, hypothalamus, hippocampus, thalamus, caudate-putamen, and globus pallidum [390].

A general caveat when analyzing the tissue distribution of the GLP-1R protein is the lack of sufficiently selective and commercially available antibodies [391]. However, Novo Nordisk has recently developed monoclonal antibodies which, through targeting of the extracellular domain of GLP-1R, allow the detection of GLP-1R in human and rodent tissues with improved selectivity [392-394]. Using these antibodies, immunohistochemical studies in tissues from humans and non-human primates confirmed the presence of GLP-1R in the pancreas with broad expression in the $\beta$-cells, weaker abundance in the acinar cells, and no expression in the duct cells [392]. GLP-1R was further detected in the arterial walls of the kidney and lung, in heart myocytes of the sinoatrial node, and in the Brunner's gland of the duodenum [392]. In heart myocytes, expression of GLP-1R is restricted to the sinoatrial node and is not observed in other cardiomyocytes [392], which aligns with the scattered expression pattern of GLP-1R in the atrium but not elsewhere in the heart [395]. Low expression of GLP-1R occurred in parietal cells and smooth muscle cells of the stomach and in myenteric plexus neurons of the gut with no expression in the liver and thyroid gland [392]. In mice, GLP-1R-positive cells were identified in several regions of the telencephalon (olfactory bulb, amygdala, preoptic area, nucleus accumbens, septum), diencephalon (hypothalamic arcuate nucleus, paraventricular nucleus, dorsomedial nucleus, the lateral hypothalamic nucleus and the supraoptic nucleus) and regions of the mesencephalon [393]. In the medulla, GLP-1R was observed in the area postrema, NTS, and lateral reticular nucleus [393].

Complementary data on the distribution of GLP-1R have been reported using other validated techniques, including in situ hybridization and highly sensitive specific antisera. Further, studies of transgenic animals controlling the expression of green fluorescent protein (GFP) downstream of the GLP-1R promoter found GLP-1R promoter activity in pancreatic $\beta$ - and $\delta$-cells, vascular smooth muscle, cardiac atrium, the gastric antrum and pylorus, enteric neurons, and vagal and dorsal root ganglia [395]. In the brain, major GLP-1R-positive regions include the area postrema, ARC, VMH, and the ventrolateral medulla with lower expression in the PVH and NTS $[395,396]$. While GLP-1R is solidly expressed in the pancreatic $\beta$ - and $\delta$-cells, most studies report absence [397-399] or very restricted expression of GLP-1R in a small subset $(<10 \%)$ of $\alpha$-cells [395]. In a more recent study, detection of GLP-1R using a fluorescent GLP-1R antagonistic peptide label (Luxendin645) located GLP-1R on the cell surface of $\sim 18 \%$ of mouse $\alpha$-cells [400]. 
7.2. Ligand-induced activation of GLP-1R

The rat and human GLP-1R protein comprises 463 amino acids with 90\% sequence homology between these species [359,360,364]. GLP1 binding to and activation of GLP-1R is a complex process that is comprehensively summarized in previous review articles [359,401]. As a class B GPCR, GLP-1R comprises seven transmembrane helices (TMH) interconnected by intracellular loops, with a C-terminal intracellular domain and a large ( $\sim 120$ amino acid) N-terminal extracellular domain (ECD) [401]. Upon biosynthesis of the receptor in the endoplasmic reticulum (ER), the N-terminal ECD of GLP-1R (and of all other class-B GPCRs) contains a short leader sequence encoding for a signal peptide. This signal peptide is of crucial importance for translocation of the receptor across the ER as well as for trafficking of the receptor to the cell surface [359,401-404]. Underlining the importance of the signal peptide in receptor trafficking, blocking the signal peptide through site-directed mutagenesis causes retention of the receptor within the ER [404]. Following translocation of the receptor across the ER, the signal peptide is enzymatically cleaved by a peptidase, leaving behind the full length GLP-1R with the N-terminal helix at the beginning of the ECD and four $\beta$-strands forming two antiparallel sheets that are connected through three disulfide bonds between six cysteine (Cys) residues [401]. The disulfide bond between Cys $^{1}$ and Cys $^{3}$ connects the $N$-terminal $\alpha$-helix of the receptor to the first $\beta$-sheet, while the disulfide bond between $\mathrm{Cys}^{2}$ and $\mathrm{Cys}^{5}$ connects two $\beta$-sheets and the disulfide bond between $\mathrm{Cys}^{4}$ and $\mathrm{Cys}^{6}$ holds the central $\beta$-sheets in proximity to the $\mathrm{C}$-terminal domain of the receptor [401,405-409].

Several studies have aimed to identify the functional GLP-1 motifs orchestrating its interaction with GLP-1R [410-412]. Analysis of the crystal structure of GLP-1R linked to GLP-1 suggests that certain residues in the $\alpha$-helical region of GLP-1 interact with residues in the $\mathrm{N}$-terminal ECD of GLP-1R $[407,409]$. These data agree with reports demonstrating a crucial role of the GLP-1R ECD for recognition by GLP1 [405,413-416]. Although these studies convincingly demonstrate the importance of the GLP-1R ECD for ligand binding, this interaction seems less important for ligand-induced activation of the receptor [401]. Accordingly, exendin (9-39) and exendin-4 have similar ligand binding affinities to the human GLP-1R, yet exendin (9-39) is not able to activate the receptor [363]. Furthermore, despite a low affinity to bind GLP-1R, [Ser(2)] exendin (1-9) is nonetheless able to activate the receptor with low potency [417]. Taken together, the ligand motifs required for receptor binding seem to differ from those motifs required for receptor activation. Indeed, for most (if not all) members of the glucagon receptor family, the activation of the receptor requires interaction of the N-terminal residues of the ligand with the transmembrane helices and extracellular loops of the receptor [401,418420]. A two-domain model has emerged and suggests that GLP-1 binds via its $\alpha$-helical and C-terminal motifs to the $\mathrm{N}$-terminal ECD of GLP-1R followed by the activation of GLP-1R through binding of the $\mathrm{N}$-terminal residues of GLP-1 with the transmembrane helices and extracellular loops of the receptor $[401,421,422]$.

A model for ligand-induced receptor conformation has also been proposed based on comparative molecular simulations using GLP-1R bound to positive and negative allosteric modulators [423]. Based on this model, a negative allosteric modulator binds and deactivates GLP$1 \mathrm{R}$ through binding between the cleft of the GLP-1R helices VI and VII, thereby pushing helix VI to an inactive state that prevents association of the receptor with the $G$ protein [423]. A positive allosteric modulator binds and activates GLP-1R through binding between helix $\mathrm{V}$ and $\mathrm{VI}$, thereby creating an intercellular binding site for the G protein [423]. Similar conclusions have been reached by analysis of the GLP-1 bound receptor conformation using cryo-electron microscopy [424] and by analyzing the crystal structure of GLP-1R bound to truncated peptide agonist [425].

\subsection{Ligand-induced biased GLP-1R agonism}

Activation of GLP-1R stimulates formation of $C A M P$ via $G_{S}$ signaling, increases intracellular $\mathrm{Ca}^{2+}$ via the $\mathrm{G}_{\mathrm{q} / 11}$ pathway, and promotes ERK $1 / 2$ signaling via recruitment of $\beta$-arrestin [426-431]. Despite binding to the same receptor, different GLP-1R ligands can engage selective pathways to elicit different cellular responses [427,431,432]. Such biased agonism is believed to have therapeutic value because certain ligands might accentuate specific signaling pathways that optimize therapeutic outcome. By assessing cAMP formation, $\mathrm{Ca}^{2+}$ accumulation and ERK1/2 phosphorylation, biased agonism was demonstrated for exendin-4 and oxyntomodulin, which, relative to GLP-1, have a bias for $\beta$-arrestin signaling $[427,431,432]$. In INS-1 cells, knockdown of $\beta$-arrestin attenuates GLP-1-stimulation of insulin secretion and decreases activation of ERK1/2 and CREB [429]. $\beta$ arrestin also promotes cell proliferation and survival via mechanisms that include activation of ERK1/2 [433]. Relative to GLP-1 and exendin4, the biased GLP-1R agonist exendin P5 favors G protein coupled signaling over recruitment of $\beta$-arrestin [434]. In lean mice, exendin P5 improves glucose handling with greater potency than exendin- 4 but has comparable efficacy to exendin-4 when analyzed more chronically in DIO and ob/ob mice [434]. In vitro comparison of receptor occupancy with ligand potency and efficacy demonstrated that the bias of exendin P5 results from enhanced efficacy in $\mathrm{G} \alpha_{s}$-mediated cAMP signaling rather than a loss of $\beta$-arrestin recruitment [435]. Functional studies of GLP-1R signaling in combination with three-dimensional modeling of the ligand-receptor complex identified the GLP-1R ECD as the key molecular trigger of biased agonism. Based on this model, the ligandspecific response of the receptor is mediated by a unique receptor conformation/stabilization that results from the specific and individual contact of the ligand with the receptor [431]. When treated with either GLP-1 or exendin P5, the greatest difference in the ligand-bound receptor structure is observed in transmembrane 1 (TM1), the extracellular portions of TM6 and TM7, and the ECL3 conformation, suggesting that these ECDs modulate biased signal transduction [435].

\subsection{GLP-1R desensitization and resensitization}

Upon ligand binding, GPCRs located on the cell surface transduce the extracellular signal to the interior of the cell. At some point, the ligandinduced receptor activation has to be terminated, and the sensitivity of the receptor to be activated by its ligand has to be restored. Data related to the molecular mechanisms leading to termination of GLP-1R activity (desensitization) and subsequent GLP-1R resensitization are incompletely understood and partially conflicting.

Desensitization of GPCRs is generally achieved by two families of serine/threonine kinases, the second-messenger-dependent protein kinases and the receptor-specific $G$ protein-coupled receptor kinases (GRKs) $[436,437]$. After ligand-induced receptor activation, these kinases phosphorylate the receptor. This induces intracellular recruitment of arrestin, which then binds to the GPCR and uncouples the receptor from its heterotrimeric $G$ proteins $[436,437]$. Another potential mode of receptor deactivation is the translocation of the ligandreceptor complex to intracellular compartments (endosomes), such that the now intracellular receptor is physically dissociated from the membrane $[436,437]$. While such ligand-induced receptor internalization has been demonstrated for many GPCRs (including GLP-1R), its importance for receptor deactivation is controversial and is potentially receptor specific. While some studies support a role of receptor 
internalization in ligand-induced receptor desensitization [438,439], no such role has been demonstrated for the $\beta 2$ adrenergic receptor $\left(\beta_{2^{-}}\right.$ ADR) [440-443] or the receptors for angiotensin ${ }_{1 A}$ [444], D1 dopamine [445], m2mACh [446], neurokinin 1 [447], histamine H2 [448], secretin [449], or GLP-1 [450-452]. However, although it may not be relevant for receptor inactivation, receptor internalization is nevertheless important for receptor resensitization. A commonly proposed model is that the phosphorylated and arrestin-bound receptor, along with a previously membrane-bound $G$ protein-coupled receptor phosphatase (GRP), is engulfed into the maturing endosome. The acidic $\mathrm{pH}$ of the endosome ( $\mathrm{pH} \sim 5-6)$ then causes a change in the receptor conformation that enables the GRP to bind and dephosphorylate the receptor. Following subsequent dissociation of the ligand and the arrestin, the re-sensitized receptor either travels back to the plasma membrane or is degraded in the lysosomes [437,453].

Ligand-induced internalization and recycling of GLP-1R has been demonstrated in Chinese hamster lung fibroblasts [453], in rat insulinoma cells [429,453,454], and in rat pancreatic BRIN BD11 cells [452]. In vitro studies using 125I-labeled GLP-1 (7-36amide) in rat insulinoma cells indicate that internalization of the ligand-GLP-1R complex is saturable and time- and temperature-dependent [454]. At the plasma membrane, GLP-1R recruitment of arrestin is observed as early as $1 \mathrm{~min}$ after stimulation with exendin-4 [452]. Surface reexpression of GLP-1R after ligand-induced GLP-1R endocytosis appears with a half-time of 15 min with no GLP-1R endocytosis upon treatment of cells with exendin (9-39) [453]. Notably, in the endosomes, the receptor-ligand complex co-localizes with adenylate cyclase. Pharmacological inhibition of GLP-1R endocytosis by dynasor attenuates CAMP formation in BRIN BD11 pancreatic $\beta$-cells and lowers PKA substrate phosphorylation, resulting in a lesser magnitude of glucose-stimulated insulin secretion [450]. These data are consistent with reports that the internalized GLP-1R is to a certain extent still capable of stimulating insulin secretion from pancreatic $\beta$-cells [451]. Continued CAMP generation after GLP-1R sequestration (internalization) is accompanied by joint translocation of the activated Gas subunit along with GLP-1R into the endosome [452]. Interestingly, generation of cAMP by the internalized GLP-1R persists after recruitment of arrestin, which indicates that arrestin recruitment is not essential for GLP-1R desensitization [452]. In contrast to these findings, siRNA mediated knockdown of $\beta$-arrestin in INS-1 cells attenuates GLP-1induced phosphorylation of CREB, ERK $1 / 2$, and IRS-2 and diminishes the insulinotropic effect of GLP-1 in conditions of low $(2.5 \mathrm{mM})$ and high glucose ( $25 \mathrm{mM})$ [429]. These studies suggest that recruitment of $\beta$-arrestin following ligand-induced activation of GLP-1R is important for the insulinotropic effect of GLP-1. However, knockdown of $\beta$ arrestin has minimal effect on GLP-1R internalization or desensitization [429]. In one recent report, exendin-4 analogs were modified on their $\mathrm{N}$-terminal end to modulate GLP-1R trafficking and/or signaling [455]. The insulinotropic efficacy of these biased-signaling molecules was inversely correlated to the receptor internalization; i.e. insulin release was greatest using molecules that retain GLP-1R at the cell surface [455].

In summary, accumulating evidence indicates that ligand-induced GLP-1R signaling is partially preserved after endocytosis of the receptor. Consistent with this, continuous CAMP formation has been demonstrated upon internalization of the receptors for parathyroid hormone [456], thyroid-stimulating hormone [457], and sphingosine 1-phosphate [458]. The exact mechanisms underlying continuation of receptor signaling after sequestration of the ligand-receptor complex seemingly involve preserved association of the receptor with the Gas subunit. The residence time of the ligand interaction with the receptor is commonly hypothesized to affect the duration an internalized receptor can continue to signal [459].

\subsection{Studies using GLP-1R-deficient animals}

Mice globally deficient for GLP-1R are viable and have no overt metabolic phenotype when fed a standard chow diet ad libitum [460462]. However, these mice are mildly hyperglycemic under conditions of fasting and display impaired glucose clearance during oral and intraperitoneal glucose tolerance tests (OGTT and ipGTT), with decreased glucose-stimulated insulin secretion [460,461] and normal pre- and postprandial levels of glucagon [461]. Although the incretin effect is diminished in GLP-1R KO mice, these mice have normal body weight and food intake on regular chow diets [460-462]. When chronically fed a high-fat diet (HFD), GLP-1R K0 mice are paradoxically leaner than their wild-type controls yet are more glucose intolerant [462]. The molecular mechanism underlying the diminished insulinotropic response of the GLP-1R KO mice to orally administered glucose likely reflects loss of GLP-1R-dependent augmentation of insulin secretion. While GLP-1 enhances proinsulin expression $[82,372,373,463,464]$, there are no differences in pancreatic insulin expression in GLP-1R K0 mice [461]. Consistent with the hypothesis that GLP-1R is the only endogenous receptor for GLP-1, either peripherally [465-467] or centrally [460] applied GLP-1R agonists fail to affect glycemic control, body weight, and food intake in mice globally deficient for GLP-1R. In summary, the most obvious metabolic phenotype of the global germline GLP-1R KO mice is diminished insulin secretion in response to oral glucose, and a paradoxical protection from diet-induced obesity despite impaired glycemic control.

\section{MOLECULAR MECHANISMS UNDERLYING GLP-1-INDUCED INSULIN SECRETION}

Induction of glucose-dependent insulin secretion by GLP-1 has been demonstrated in vivo in numerous species including rodents [468471] and humans [83] as well as in the isolated perfused pancreas of rats [81,472-474], dogs [473], pigs [80,475], and humans [77], and in isolated islets [476] and cultured pancreatic cell lines [82,370$373,472,477-479]$. GLP-1 (7-37) is roughly 100 -fold more potent than glucagon to stimulate the secretion of insulin [480]. Nevertheless, intra-islet glucagon levels are much higher than circulating levels, and studies in mice highlight the importance of glucagon, acting through the GLP-1 receptor in the control of glucose-stimulated insulin secretion $[173,365]$. The key importance of GLP-1 and GIP in stimulating insulin secretion under conditions of hyperglycemia is the finding that oral glucose-induced insulin secretion is more impaired in mice that lack both incretin hormone receptors [481-483].

\subsection{Acute insulinotropic effects of GLP-1}

In $\beta$-cells, binding of GLP-1 to its receptor leads to activation of adenylate cyclase $(\mathrm{AC})$ and subsequently to an increase in cAMP (Figure 5) [82]. Overexpression of GLP-1R in rat insulinoma RIN104638 cells results in elevated levels of basal cAMP [484]. While both GLP1 (1-37) and GLP-1 (7-37) stimulate insulin secretion, GLP-1 (7-37) is efficacious at a lower dose. At a dose of $5 \mu \mathrm{M}$, both GLP-1 (1-37) and GLP-1 (7-37) increase CAMP levels in RIN1046-38 cells but at a dose of $5 \mathrm{nM}$, only GLP-1 (7-37) enhances cAMP levels [82]. Consistent with the increase in CAMP, GLP-1 stimulation of insulin mRNA is substantially greater upon treatment of RIN1046-38 cells with GLP-1 (7-37) relative to treatment with GLP-1 (1-37) [82]. Further supporting the greater insulinotropic potency of GLP-1 $(7-37)$ relative to GLP-1 (1-37), GLP-1 (7-37) stimulates insulin release in the 


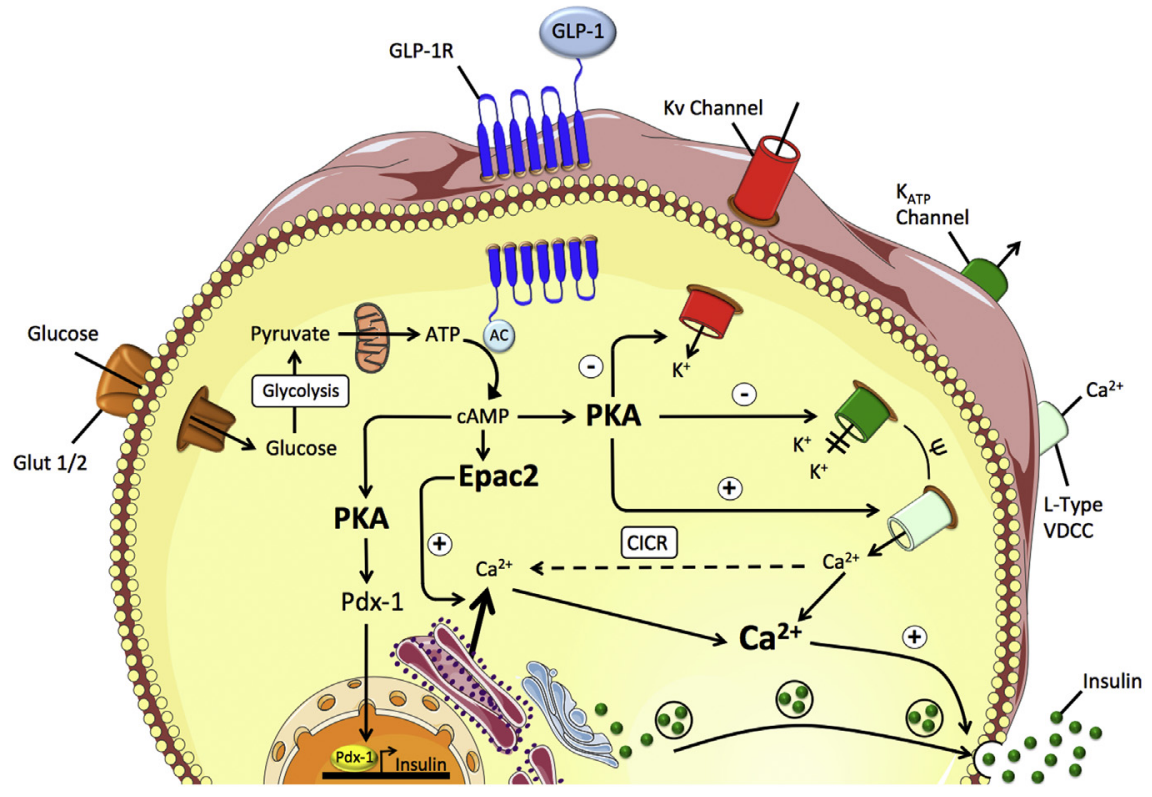

Figure 5: Schematic on GLP-1 mediated insulin secretion in the $\beta$-cell. GLUT1/2: glucose transporter 1/2; AC: adenylate cyclase; PKA: protein kinase A; Epac2: exchange protein activated by CAMP; Pdx-1: pancreatic and duodenal homeobox 1; CICR: calcium-induced calcium release. For further explanations, please see text.

perfused rat pancreas at a concentration of $50 \mathrm{pM}$ whereas treatment with GLP-1 (1-37), even at a dose of $0.5 \mu \mathrm{M}$, has no effect on insulin secretion [81]. Underlining the role of cAMP in the insulinotropic effect of GLP-1, enhanced CAMP hydrolysis through overexpression of the cyclic nucleotide phosphodiesterase 3B (PDE3B) diminishes GLP-1induced insulin secretion [485]. Insulin and IFG-1 increase levels of PDE3B, and treatment of hamster clonal $\beta$-cells (HIT-T15) with IGF-1 deteriorates the insulinotropic effect of GLP-1 [486]. Transgenic mice overexpressing PDE3B under control of the rat insulin 2 promoter are glucose intolerant and have impaired insulin secretion following an intravenous glucose challenge [487]. In summary, there is substantial evidence indicating that CAMP is an important second messenger driving the acute insulinotropic effect of GLP-1.

The GLP-1-induced rise in CAMP leads to activation of PKA and enhanced signaling via exchange proteins directly activated by CAMP (Epac) (Figure 5) [488-490]. cAMP activates PKA, which phosphorylates the $\beta_{2}$ subunit of the L-type VDC channels and potentially the Kir6.2 and SUR1 subunits of the $K_{\text {ATP }}$ channels [491]. This consequently increases the sensitization of the $K_{A T P}$ channels to ATP, leading to closure of the $\mathrm{K}_{\text {ATP }}$ channels, depolarization of the cell membrane, and opening of VDC channels (Figure 5). The subsequent $\mathrm{Ca}^{2+}$ influx promotes exocytosis of the insulin granules and acute secretion of insulin into the circulation. At the same time, the GLP-1-activated PKA inhibits voltage-gated $\mathrm{K}^{+}\left(\mathrm{K}_{\mathrm{v}}\right)$-channels, preventing membrane repolarization and boosting $\mathrm{Ca}^{2+}$ influx via prolonged opening of the VDC channels (Figure 5) [492]. Inhibition of PKA in isolated islets attenuates GLP-1-induced insulin secretion [490].

The acute insulinotropic effect of GLP-1 does not fully depend on PKA signaling [493]. Up to $50 \%$ of the GLP-1-induced insulin release can be mediated through signaling via Epac $[489,494,495]$. Similar to PKA, members of the Epac family contain an evolutionarily conserved cAMP binding domain which enables the members of this family (Epac1 and Epac2) to regulate diverse biological functions in a cAMP-dependent manner [496]. Both Epac1 and Epac2 are expressed in the pancreas
$[495,497]$. In $\beta$-cells, the Epac proteins stimulate $\mathrm{Ca}^{2+}$ release from the endoplasmic reticulum (ER), increasing insulin secretion by increasing the intracellular pool of $\mathrm{Ca}^{2+}$ [488]. cAMP activates Epac2 through direct binding. In high glucose conditions where there is an enhanced $\mathrm{Ca}^{2+}$ influx into the $\beta$-cells via the VDC channels, Epac2 opens RYR $\mathrm{Ca}^{2+}$ channels in the ER, further increasing intracellular $\mathrm{Ca}^{2+}$ levels, and potentiates insulin exocytosis (Figure 5) [489]. The GLP-1/Epac2 mediated opening of the RYR $\mathrm{Ca}^{2+}$ channels in the ER depends on the simultaneous $\mathrm{Ca}^{2+}$ influx via the VDC channels. This process, generally referred to as calcium-induced calcium release (CICR) (Figure 5) [489,498-500], ensures that the insulinotropic action of GLP-1 is highly dependent on the ambient glucose concentration. Accordingly, in the isolated perfused rat pancreas, GLP-1 fails to stimulate insulin release at glucose concentrations $<2.8 \mathrm{mM}$, but amplifies insulin release at glucose concentrations $>6.6 \mathrm{mM}[82,480]$. Consistent with this, clinical studies demonstrate that GLP-1R agonism improves glycemic control in patients with type 2 diabetes but with little risk of hypoglycemia when GLP-1 is used without accompanying sulfonylurea therapy [501-507].

Beyond its ability to increase the intracellular pool of $\mathrm{Ca}^{2+}$, GLP-1 also affects insulin secretion via CAMP-mediated regulation of exocytosis (Figure 6). In isolated murine $\beta$-cells, GLP-1 stimulates exocytosis of the insulin granules and this can be blocked by co-treatment of GLP-1 with the adenylate cyclase inhibitor MDL 12330A [508]. As reviewed previously [508], the insulin granules are organized in distinct pools that vary in their release competence. Most granules ( $>99 \%$ ) belong to the immature "reserve" pool that must undergo a series of maturation processes to acquire release-competence. Only $0.5-1 \%$ of the granules belong to the readily releasable pool (RRP), from which only a subset, the immediately releasable pool (IRP), is located in the vicinity of the $\mathrm{Ca}^{2+}$ channels and is released with minimal time delay upon opening of the $\mathrm{Ca}^{2+}$ channels [508]. cAMP enhances insulin exocytosis by promoting maturation of the granules and thus by increasing the pool of release-competent (RRP/RP) granules [508-510]. 

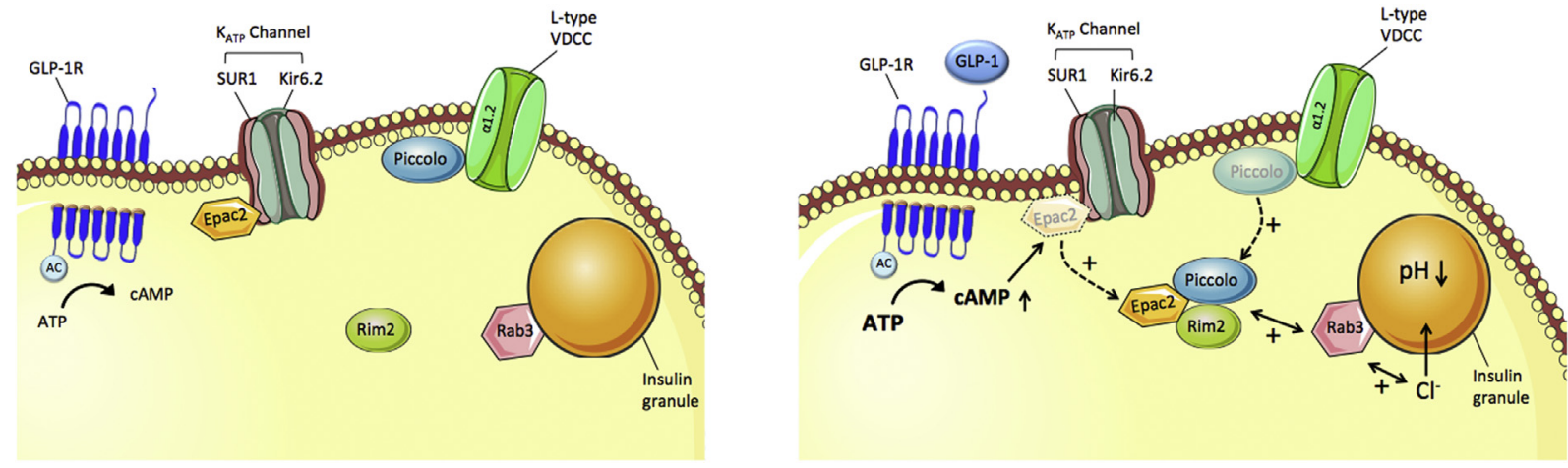

Figure 6: Schematic on GLP-1 mediated exocytosis of the insulin granules in the $\beta$-cell. For further explanations, please see text.

CAMP regulates exocytosis via PKA-dependent and -independent mechanisms. GLP-1-stimulation of exocytosis is partially inhibited when isolated mouse $\beta$-cells are treated with the PKA inhibitor H89 but is fully inhibited upon co-treatment with $\mathrm{H} 89$ with an antiserum against Epac2 (a.k.a. cAMP-GEFI) [508]. Epac2 binds to the nucleotide binding domain-1 of the SUR1 subunit of the $\mathrm{K}_{\text {ATP }}$ channel and this interaction is aborted when levels of cAMP increase (Figure 6) [511]. Apart from binding SUR1, Epac2 also binds to a series of molecules that potentially participate in transport, priming and fusion of the insulin vesicles, including Rab3, Rim2, and the $\mathrm{Ca}^{2+}$ sensor Piccolo [493,512,513]. Epac2 interaction with Rim2 and Piccolo is crucial for cAMP-induced $\mathrm{Ca}^{2+}$-dependent insulin secretion and is not blocked by inhibition of PKA [493,512,513]. A hypothetical model for the regulation of exocytosis by Epac2 is that the GLP-1-induced rise in cAMP leads to a conformational change of Epac2, which then dissociates from SUR1 and stimulates dissociation of Rim2 from the $\alpha 1.2$ subunit of the Ltype $\mathrm{Ca}^{2+}$ channel (Figure 6). The dissociated Epac2 then dimerizes with Rim2 and Piccolo in a $\mathrm{Ca}^{2+}$-dependent manner followed by induction of exocytosis of the insulin granules through binding of the Epac2/Rim2/Piccolo complex to Rab3 which is located at the membrane of the insulin granules (Figure 6) [508,511]. Another PKAindependent mechanism of GLP-1 stimulation of insulin secretion includes PKC-dependent membrane depolarization via activation of transient receptor potential cation channel subfamily $M$ member 4 (Trpm4) and Trpm5 [514]. In murine and human islets, GLP-1, via phospholipase $\mathrm{C}(\mathrm{PLC})$, leads to an activation of PKC, followed by membrane depolarization and stimulation of insulin secretion. Membrane depolarization by GLP-1 was mimicked by the PKC activator phorbol myristate acetate (PMA) and was not affected by treatment with either the PKA inhibitor myr-PKI, the $\mathrm{K}_{\text {ATP }}$ channel blocker tolbutamide, or the L-type $\mathrm{Ca}^{2+}$ channel inhibitor isradipine [514]. The PKC-dependent effect of GLP-1 on membrane depolarization was mediated by activation of Trpm4 and Trpm5, and GLP-1 failed to increase electrical activity and insulin secretion in islets isolated from Trpm4 and Trpm5 KO mice [514]. Another recent report suggests that incretin-stimulation of insulin secretion requires incorporation of glutamate in the insulin granules. In murine pancreatic Min6-K8 cells, prevention of cytosolic glutamate production by $\mathrm{KO}$ of a key component of the malate-aspartate shuttle or triple $\mathrm{KO}$ of the vesicular glutamate transporters Slc17a6, Slc17a7, and SIc17a8 impaired GLP-1-induced insulin secretion [515].

GLP-1 also lowers blood glucose in patients with type 1 diabetes, e.g. via its ability to slow down gastric emptying, indicating that not all of GLP-1s glycemic effects derive from its action on the $\beta$-cell or insulin action [516,517]. Post-prandial levels of portal blood glucose are elevated and insulin levels decreased in rats with knockdown of GLP$1 \mathrm{R}$ in vagal afferent neurons, suggesting that GLP-1 also regulates blood glucose via activation of vagal afferent GLP-1 receptors [518]. These data are consistent with a recent report showing that GLP-1 stimulation of insulin secretion requires GLP-1R and neuronal nitric oxide synthase in the enteric nervous system [519]. Moreover, mice with targeted inactivation of GLP-1R in autonomic neurons targeted by Phox2b, including the nodose ganglion, exhibit impaired glucose homeostasis, accelerated gastric emptying, and increased levels of GLP1 , glucagon, and insulin, supporting the importance of vagal afferent GLP-1Rs for metabolic homeostasis [520].

\subsection{Effects of GLP-1 on (Pro-)insulin synthesis}

In addition to the ability of GLP-1 to stimulate insulin secretion via signaling through PKA and Epac2, GLP-1R agonism also increases glucose metabolism by promoting insulin synthesis (Figure 5). GLP-1 stimulation of insulin gene expression was first reported by Daniel Drucker in rat insulinoma RIN1046-38 cells [82] and was subsequently confirmed in several independent studies [372,373,463,464]. In RIN 1046-38 cells, the GLP-1-induced rise in insulin mRNA is accompanied by an increased expression of GLUT1 and hexokinase1 [464]. Treatment with the transcription inhibitor actinomycin D blocks GLP-1 stimulation of GLUT1 and hexokinase1 expression in these cells without affecting GLP-1 induction of insulin expression [464]. These data led to the hypothesis that the increased insulin mRNA levels occurring upon GLP-1 exposure might be due to stabilization of the mRNA, whereas the elevated expression of GLUT1 and hexokinase are due to direct transcriptional stimulation by GLP-1 [464]. Nevertheless, GLP-1 also activates proinsulin gene transcription in islet insulinoma cells [372,521]. Notably, induction of proinsulin expression by either GLP-1 or forskolin can be blocked by galanin, suggesting that GLP-1 stimulation of insulin expression is cAMP dependent [373].

GLP-1-induced stimulation of insulin synthesis is initiated by $\mathrm{Pdx} 1$, a transcription factor implicated in development of the pancreas [490] and in MODY type-4 diabetes [522,523]. GLP-1 induced activation of PKA increases Pdx1 expression and translocation of Pdx1 to the nucleus, where Pdx1 binds to the insulin promoter to initiate insulin expression and synthesis (Figure 5) [490,524]. As demonstrated in RIN1046-38 cells, PKA activation of Pdx1, but not glucose-induced Pdx1 nuclear translocation, can be blocked either by PKA inhibition or by using a cAMP antagonist, implying that GLP-1 regulates the PKA/ Pdx1 axis in a cAMP-dependent manner [490]. Accordingly, GLP-1 effects on Pdx1 can be mimicked by treatment of RIN1046-38 cells 
with either forskolin or the cAMP analog 8-Bromo-cAMP [490]. In summary, GLP-1 stimulates both the synthesis and secretion of insulin via multiple cAMP-dependent pathways.

\section{GLP-1 EFFECTS ON $\beta$-CELL PROLIFERATION AND APOPTOSIS}

The prevalence of type 2 diabetes correlates with excess body weight and also increases with age [525]. The progression to type-2 diabetes is invariably associated with a decline in functional $\beta$-cell mass [526530]. Decreased $\beta$-cell proliferative capacity is also age-dependent in rodents and humans $[526,531-537]$. The replication rate of human $\beta$ cells is greatest in young childhood and puberty but declines with increasing age [526,531,533,536]. Collectively, these observations suggest that age-related changes in $\beta$-cell neogenesis and replication might be causally linked to the development of type 2 diabetes $[525,532,538]$. A role for $\beta$-cell dedifferentiation has also been proposed [539].

Agonists of the GLP-1 receptor improve glycemic control via both their acute insulinotropic action and, under certain circumstances, also by chronic action to preserve $\beta$-cell mass through stimulation of $\beta$-cell proliferation and inhibition of apoptosis (Figure 7) [540-545]. While inconsistent results have been reported, the treatment duration, age of the animal, and species under investigation as well as diet-composition might affect the ability of GLP-1R agonism to improve $\beta$-cell replication [540]. GLP-1R regulation of $\beta$-cell proliferation and apoptosis seems to engage mechanisms that include signaling via $\mathrm{Pdx} 1$. Exendin-4 stimulates $\beta$-cell proliferation and inhibits $\beta$-cell apoptosis in wildtype mice but not in mice with $\beta$-cell specific inactivation of $\mathrm{Pdx} 1$ [542]. GLP-1R agonism also promotes $\beta$-cell growth and survival by stimulating the expression of the insulin receptor substrate 2 (Irs2) via mechanisms that include activation of CREB [546]. Irs2 is a substrate of the IGF1 and insulin receptor tyrosine kinases that promote growth, function and survival of the $\beta$ cells [547]. Consistent with this role of Irs2, increased expression of Irs2 in $\beta$-cells improves insulin secretion in obese mice and protects against STZ-induced $\beta$-cell destruction (as reviewed in [547]). Mice deficient for Irs2 [544], or transgenic mice deficient in CREB activity [546], are hyperglycemic due to severe $\beta$-cell destruction and enhanced $\beta$-cell apoptosis. Exendin-4 improves Irs2 function by enhancing CREB phosphorylation in vitro and in vivo [546]. Chronic administration of exendin- 4 is unable to prevent $\beta$-cell loss in mice that are lacking Irs2 [544], demonstrating the requirement of Irs2 to GLP-1-mediated $\beta$-cell plasticity. Stimulation of $\beta$-cell proliferation secondary to stimulating GLP-1R is usually only observed in young animals (during periods characterized by preserved proliferative capacity), and not in older rodents [548]. Solid clinical proof of slowed diabetes progression or enhanced $\beta$-cell mass has not been provided in type-2 diabetic patients treated with any GLP-1 receptor agonist [549].

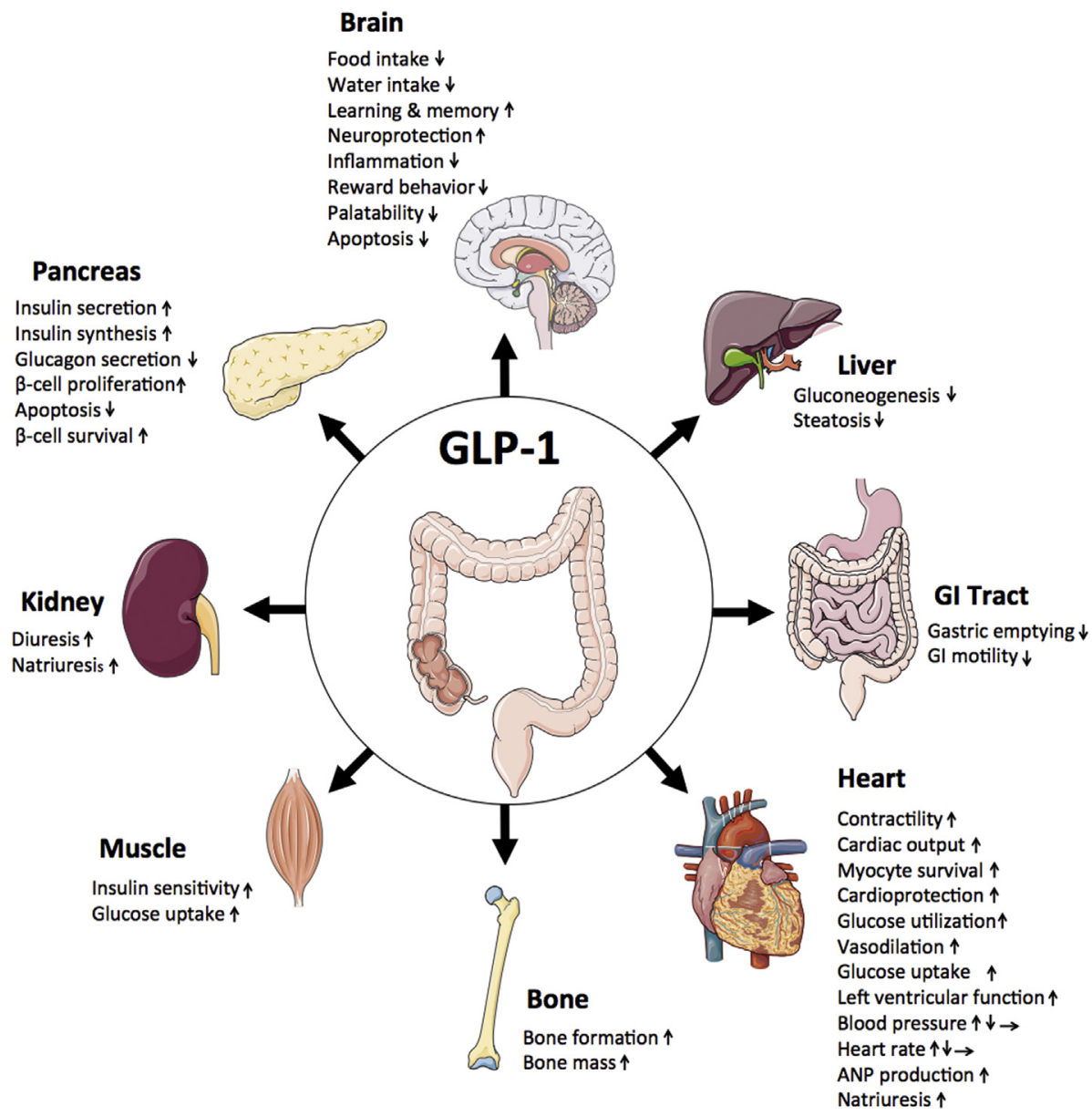

Figure 7: Schematic on the metabolic effects of GLP-1. The shown effects include direct and indirect GLP-1 effects on metabolism. For further explanations, please see text. 
The anti-apoptotic effect of GLP-1R agonism has been demonstrated in vivo in mice $[543,550,551]$ and rats $[552,553]$ as well as in several rodent $[550,551]$ and human $[554,555]$ cell lines, purified rat $\beta$-cells [543], and humans [556]. STZ-induced $\beta$-cell apoptosis is reduced upon administration of exendin-4 [543] or GLP-1 (7-36 amide) [551] and treatment with exendin-4 diminishes hyperglycemia induced by STZ [543]. STZ-induced $\beta$-cell apoptosis is accelerated in mice deficient for the GLP-1 receptor [543]. In isolated rat $\beta$-cells, treatment with exendin- 4 reduces the apoptotic effect induced by treatment with pro-apoptotic cytokines, such as IL1b, TNFa, and interferon gamma [543]. In mouse pancreatic $\beta$ TC- 6 cells, liraglutide enhances $\beta$-cell survival via stimulation of anti-apoptotic signaling mechanisms that include stimulation of PI3 kinase-dependent phosphorylation of AKT, leading to inactivation of the pro-apoptotic protein BAD and silencing of Fox01 [550].

An innovative approach to assess human $\beta$-cell replication in vivo was recently established by the group of Alvin Powers [557]. In this model, human $\beta$-cells were grafted under the renal capsule of lean diabetic immunodeficient NOD scid gamma mice. Using this model, exendin-4 was found to enhance $\beta$-cell proliferation in juvenile but not adult human islets [557]. Notably, the age-dependent decline in the exendin4 induced $\beta$-cell proliferation was not related to changes of GLP-1R expression, as also indicated by preservation of exendin-4-induced insulin secretion in the adult islets [557]. However, in juvenile but not adult human islets, exendin-4 stimulated calcineurin/NFAT signaling and enhanced expression of proliferation-promoting factors such as NFATC1, FOXM1, and CCNA1 [557]. [557]. Collectively, these data suggest that the sensitivity of $\beta$-cell proliferative mechanisms to respond to GLP-1R agonism declines with increasing age [557].

\section{IMPAIRED RESPONSIVENESS OF GLP-1 TO STIMULATE INSULIN SECRETION}

GLP-1-stimulated insulin secretion has an estimated heritability of 0.53 , as demonstrated in twin studies [558]. The first evidence for an influence of genetic background on GLP-1-induced insulin secretion was found for variation in the transcription factor 7-like 2 (TCF7L2) [559]. Infusion of GLP-1 during a classical hyperglycemic clamp in non-diabetic individuals results in a marked reduction of insulin secretion in carriers of the TCF7L2 risk allele of SNP rs7903146. Diminished insulin secretion in response to treatment with GLP-1 has been replicated in other studies [560-562].

In addition to TCF7L2, several other genetic loci have been identified to be associated with reduced GLP-1-induced insulin secretion, including the loci for GLP-1R [563], wolfram syndrome 1 (WFS1) [564] and chymotrypsinogen B1/2 (CTRB1/2) [565]. The molecular mechanisms leading to diminished GLP-1 responsiveness associated with genetic variation in TCF7L2 most likely includes alterations of the WNT signaling pathway and associated $\beta$-cell proliferation and insulin gene expression [566]. Interestingly, genetic variation in a transcriptional regulator of the insulin gene nuclear receptor subfamily 4 group $A$ member 3 (Nor-1) is able to rescue TCF7L2-mediated GLP-1 resistance [567]. Impairment of proinsulin conversion may be another mechanism for TCF7L2 associated diminished GLP-1 efficacy [568]. Another plausible mechanistic explanation for a diminished "incretin effect" is the TCF7L2-dependent repression of GLP-1R and GIPR expression on the $\beta$-cells [569]. The association between WFS1 variants and impaired incretin action may result from alterations of endoplasmic reticulum homeostasis and, consequently, $\beta$-cell dysfunction [566]. Recently, using an untargeted integrative genomics approach, a set of genes that contained variants associated with GLP- 1-stimulated insulin secretion were described, which at the same time have the potential to physically interact in the $\beta$-cell and are enriched for pathways that are important for insulin secretion [570]. Finally, the GLP-1 effects on insulin secretion are dependent on the metabolic state of the individual. Incretin action may also be reduced during hyperglycemia and in some individuals with diabetes, prediabetes and insulin resistance [571].

In summary, a reduced incretin effect on insulin secretion is associated with both genetic and metabolic alterations. The presence of both hyperglycemia and genetically determined GLP-1 resistance can potentiate the impairment of GLP1-induced insulin secretion [572].

\section{GLP-1 EFFECTS ON GLUCAGON SECRETION}

GLP-1 also decreases blood glucose by suppressing glucagon secretion (Figure 7) $[475,573,574]$. GLP-1 inhibition of glucagon secretion has been demonstrated in vivo in numerous species including mice [575], dogs [576], and humans [516,517,573,577-581], as well as in the isolated perfused pancreas of rats [473,474,582], dogs [473], and pigs [475] and in isolated intact murine islets [583]. Clamp studies in patients with type 2 diabetes indicate that GLP-1 inhibition of glucagon secretion is equally important for lowering blood glucose as GLP-1 stimulation of insulin release [578].

The mechanisms underlying GLP-1 suppression of glucagon secretion are complex. In the isolated perfused pig pancreas, GLP-1 dosedependently stimulates the secretion of somatostatin [475] which is a potent suppressor of glucagon secretion [584]. Somatostatin inhibits glucagon secretion via paracrine mechanisms and, when blocked, stimulates glucagon release in isolated rat islets [585,586]. In the isolated perfused rat pancreas, co-infusion of GLP-1 with a specific somatostatin receptor 2 (SSTR2) antagonist (PRL-2903) abolishes the GLP-1-induced suppression of glucagon secretion [582]. While these data point to a prominent role of somatostatin in mediating GLP-1 suppression of glucagon secretion, treatment of isolated murine islets with the SSTR2 antagonist CYN154806 does not fully inhibit GLP1 's ability to suppress glucagon secretion [583]. Consistent with the conclusion that GLP-1 suppression of glucagon secretion does not fully depend on somatostatin, GLP-1 effects on glucagon secretion can be mimicked by forskolin-induced changes in CAMP. Accordingly, treatment of isolated murine islets with low concentrations of forskolin (1$10 \mathrm{nM}$ ) suppresses glucagon secretion by up to $60 \%$, while high concentrations of forskolin $(0.1-10 \mu \mathrm{M})$ stimulate glucagon release [583]. Notably, the PKA inhibitor 8-Br-Rp-cAMPS attenuates the inhibitory effect of GLP-1 on glucagon secretion, suggesting that GLP-1 suppression of glucagon secretion is PKA-dependent [583]. In intact murine islets, blockade of $\mathrm{N}$-type $\mathrm{Ca}^{2+}$ channels with $\omega$-conotoxin, but not L-type $\mathrm{Ca}^{2+}$ channels using nifedipine, abolishes low glucose (1 $\mathrm{mM}$ ) stimulation of glucagon secretion and blunts the GLP-1mediated inhibitor effects. In summary, these data indicate that GLP-1 may inhibit $\alpha$-cell glucagon secretion via PKA-dependent modulation of $\mathrm{N}$-type $\mathrm{Ca}^{2+}$ channel activity in addition to its paracrine action via somatostatin [583].

GLP-1 may also inhibit glucagon secretion indirectly via its insulinotropic effect on the $\beta$-cells. Accordingly, as described in recent review articles [4,574], GLP-1 stimulates both the $\delta$-cells to secrete somatostatin and the $\beta$-cells to secrete insulin, amylin, zinc, and GABA, all of which suppress the release of glucagon. In $\alpha$-cell-derived IN-R1G9 cells, insulin inhibits glucagon release via activation of phosphatidylinositol 3-kinase (PI3K). Inhibition of PI3K by wortmannin offsets the ability of insulin to suppress glucagon secretion [587]. In $\alpha$-cells, insulin further enhances translocation of GABA-A receptors [588], and 
GABA released from $\beta$-cells enhances glucose inhibition of glucagon secretion [589]. Insulin is co-crystalized with $\mathrm{Zn}^{2+}$ in the secretory granules of the $\beta$-cells $[590,591]$ and $\mathrm{Zn}^{2+}$ is co-secreted with insulin under conditions of hyperglycemia [592,593]. $\mathrm{Zn}^{2+}$ acts in a paracrine manner on the $\alpha$-cells to inhibit the secretion of glucagon [593-595]. Interestingly, disruption of the intrapancreatic infusion of $\mathrm{Zn}^{2+}$ but not of $\mathrm{Zn}^{2+}$ free insulin accelerates glucagon secretion in rats made diabetic by STZ, indicating that $\mathrm{Zn}^{2+}$ but not insulin is the main stimulus underlying the $\mathrm{Zn}^{2+}$-insulin inhibition of glucagon secretion [595]. Consistent with this, glucagon secretion is inhibited upon treatment of $\alpha$-TC cells with $\mathrm{Zn}^{2+}$ [596]. As demonstrated in isolated rat $\alpha$-cells, intact islets and the perfused rat pancreas, $\mathrm{Zn}^{2+}$ inhibits pyruvate-induced glucagon secretion via opening of $\mathrm{K}_{\text {ATP }}$ channels and inhibition of $\alpha$-cell electrical activity [593]. In summary, there is credible evidence indicating that $\mathrm{Zn}^{2+}$ that is co-secreted with insulin from the $\beta$-cells plays a prominent role in inhibiting release of glucagon [593-595]. Amylin, which is also co-secreted with insulin, also affects glucagon release from the $\alpha$-cells. Amylin dose-dependently suppresses arginine-mediated glucagon secretion in rats [597] whereas pharmacological inhibition of amylin signaling enhances glucagon secretion [598]. Pramlintide, a synthetic amylin receptor agonist, improves glycemic control in diabetic patients via inhibition of postprandial glucagon secretion and inhibition of gastric emptying $[599,600]$. Interestingly, amylin does not affect glucagon secretion in isolated islets [601] nor in the perfused rat pancreas [602,603], suggesting that amylin regulation of glucagon is not cell autonomous. Notably, while GLP-1 can potentially affect glucagon secretion via its stimulatory effect on the $\beta$-cells in normal physiological conditions, GLP-1 also inhibits glucagon release in type 1 diabetes, thus demonstrating that GLP-1 inhibition of glucagon secretion does not fully depend on the $\beta$-cell secretome [516].

In summary, GLP-1 potently inhibits glucagon secretion. This occurs in part by a paracrine stimulatory effect on the islets to secrete somatostatin, and perhaps insulin, $\mathrm{Zn}^{2+}, \mathrm{GABA}$, and amylin, at elevated glucose concentrations. GLP-1 likely suppresses glucagon secretion via endocrine rather than direct mechanisms. Most studies report the absence [397-399] or very restricted expression of the GLP-1 receptor in a small subset (10\%) of $\alpha$-cells [395], and treatment of isolated rat $\alpha$-cells with GLP- 1 enhances rather than inhibits the release of glucagon [604-606]. GLP-1 may nevertheless also directly inhibit glucagon secretion, because $\alpha$-cell-specific GLP-1R K0 mice have elevated non-fasting glucagon levels relative to wildtype controls and female $\alpha$-cell-specific GLP-1R KO mice are mildly glucose intolerant with increased secretion of glucagon upon peripheral administration of glucose [607]. Further supporting a direct GLP-1 inhibition of glucagon secretion is the fact that such secretion is not prevented in isolated human islets treated with the insulin receptor antagonist S961 or the somatostatin receptor 2 antagonist CYN154806 [608].

\section{CARDIOVASCULAR EFFECTS OF GLP-1}

The presence of GLP-1R mRNA in the heart was first demonstrated in rats [380] and humans [381,609]. Expression was subsequently confirmed at the protein level $[201,610]$ and histologically using mice that express GFP under control of the GLP1-R promoter. In these mice scattered GLP-1R expression was detected in cardiomyocytes of the atrium but not in the ventricles, and widespread expression was detected in smooth muscle cells of coronary vessels [395]. As discussed in a comprehensive review article [611], the anatomical location of GLP-1R within the cardiovascular system has speciesrelated differences. In mice, GLP-1R immunoreactivity using multiple different antisera is found in the cardiomyocytes, endocardium, microvascular endothelium, and the smooth muscle cells in the coronary arteries [201]. In humans, the presence of GLP-1R has primarily been demonstrated in the endothelium, the coronary arteries and the smooth muscle cells $[381,612]$. Nevertheless, there is little concordance between the available localization data from immunohistochemistry vs. detection of GLP-1R mRNA in situ hybridization. In view of the many specificity problems associated with antibodies against the GLP-1 receptor, some of the incongruent findings may also result from lack of antisera specificity. Using well-characterized antibodies, expression was noted in the non-human primate and human sinoatrial node [392], which would be consistent with the effect of GLP-1 on heart rate. Surprisingly, in one recent study, robust expression of the GLP-1 receptor mRNA was also reported in human cardiac ventricles, but the exact localization of the GLP-1R protein could not be determined [613].

Although best known for its insulinotropic and weight lowering action, GLP-1R agonism also confers a series of beneficial effects on the cardiovascular system in rodents. These include an increase of cardiomyocyte survival via inhibition of apoptosis [614,615], amelioration of endothelial dysfunction [612,616], improvement of regional, and global cardiac output following injury and heart failure [614,617-619] (Figure 7). Reduction of blood pressure is also reported in hypertensive humans [620]. Prolonged treatment with liraglutide further improves cardiovascular outcome in patients with T2DM [621]. Of note, GLP-1 improvement of cardiovascular performance is, at least in part, independent of its ability to decrease body weight and to improve lipid metabolism, and GLP-1 may promote its cardiac effects via direct action at GLP-1R as well as indirect mechanisms independent of cardiac GLP-1R action [201,611,622].

\subsection{Effects of GLP-1 on cardiac performance after cardiac injury} Endothelial dysfunction is a common co-morbidity associated with insulin resistance and T2DM. Treatment of type- 2 diabetic patients that present coronary artery disease with GLP-1 improves endothelial function without affecting insulin resistance [612]. Similar findings have been reported for GLP-1 treatment of type-1 diabetes [623] but GLP-1 improvement of endothelial function seems to be related to native GLP-1 and is not observed when GLP-1R agonists are compared head-to-head with equally glucose lowering medications.

GLP-1 improvement of endothelial function, assessed by flowmediated vasodilation, was also demonstrated during a hyperglycemic clamp in patients with T2DM and normoglycemic controls [616]. In rats, GLP-1 (7-36amide) and, perhaps paradoxically, the GLP-1R antagonist exendin $(9-39)$, dose-dependently cause relaxation of the aorta, potentially via elevation of CAMP formation and activation of $\mathrm{K}_{\text {ATP }}$ channels [624].

In dogs with acute myocardial infarction, infusion of GLP-1 improves cardiac performance by increasing myocardial glucose uptake and by enhancing left ventricular function [617]. In the same animal model, infusion of GLP-1 limited myocardial stunning (ventricular dysfunction without myocardial necrosis) following reperfusion [618]. These data are in agreement with non-controlled clinical studies reporting that $72 \mathrm{~h}$ of native GLP-1 infusion improved regional and global left ventricular performance in patients with acute myocardial infarction and severe systolic dysfunction [619]. In mice, 7-day treatment with liraglutide prior to induction of myocardial infarction increases survival and reduces cardiac injury and infarction size, while improving cardiac output [614]. In the isolated perfused rat heart, acute GLP-1R agonism protects from ischemia/reperfusion injury [625,626], an effect that can be blocked by pretreatment with either exendin (9-39), the cAMP 
inhibitor Rp-cAMP, the PI3 Kinase inhibitor LY294002 or the MAP Kinase inhibitor U0126 [625]. The cardioprotective effect of GLP-1R agonism seems to be, at least in part, independent of GLP-1induced weight loss, dependent on the canonical GLP-1R, and accompanied by enhanced expression of cardioprotective genes in the heart, including Akt, GSK3 $\beta$, PPAR $\Delta$, NRF-2, and HO-1 [614]. Treatment of primary neonatal mouse cardiomyocytes with liraglutide increases CAMP formation and reduces apoptosis, as indicated by inhibition of TNFa induction of caspase 3 activity [614]. GLP-1R agonism has direct cell autonomous anti-apoptotic effects in the rodent heart, and GLP-1 reduces hypoxia-induced apoptosis in isolated neonatal rat cardiomyocytes, and inhibition of PI3K using LY29400 or MAPK using U0126 blocks the anti-apoptotic effect of GLP-1 [615]. GLP-1 has different effects on left ventricular function and cardiac contractility under healthy vs. pathological conditions. GLP-1-induced improvement of left ventricular function has been reported in dogs with cardiomyopathy [617], in patients with acute myocardial infarction [619], and in the isolated mouse heart [201]. At least part of the improvement in cardiac output may be driven by an increase in heart rate. Other studies, however, report failure of GLP-1 to directly accelerate contraction in isolated adult rat cardiomyocytes, notably despite GLP-1 stimulation of cAMP accumulation [627]. Further, treatment of the healthy isolated rat heart with GLP-1 has been reported to reduce left ventricular contractility despite GLP-1 stimulation of myocardial glucose uptake [628]. However, as demonstrated in the same study, GLP-1 enhances recovery of the heart after ischemia and improves left ventricular function and myocardial glucose uptake [628]. GLP-1R K0 mice have increased baseline left ventricular developed pressure (LVDP) during perfusion [201]. Mice deficient for GLP-1R have decreased resting HR and an increased thickness of the left ventricular wall relative to wildtype controls [201,629], as well as reduced cardiac contractility when challenged with insulin or epinephrine [629]. Collectively, these data indicate that GLP-1 improves cardiac performance and output in the post-ischemic heart but otherwise may decrease cardiac output under non-pathological conditions. Although mice with cardiomyocyte-specific deletion of GLP-1R have lower basal heart rate (HR), they fully retain the cardioprotective potential of GLP-1R agonism following left arterial descending (LAD) coronary artery occlusion [630]. These data suggest that cardiomyocyte GLP-1R signaling is required for regulation of $H R$ but is unrelated to the cardioprotective effects of GLP-1R agonism following cardiac injury.

\subsection{Cardiometabolic effects of GLP-1(9-36amide)}

Accumulating evidence indicates that some cardiac effects of GLP-1 (7-36amide) are actually mediated via the DPP-4-generated GLP-1 (9-36amide) and its smaller degradation products and, thus, are independent of GLP-1R signaling. At a dose of $0.3 \mathrm{nmol} / \mathrm{L}$, pretreatment with GLP-1 (7-36amide) improves recovery of LVDP after ischemia/ reperfusion (l/R) injury in both WT and GLP-1R KO mice [201]. Interestingly, a $>10$ fold greater dose of exendin- 4 is needed relative to GLP-1 (7-36amide) to achieve a similar effect in WT mice but still with lower efficacy relative to GLP-1 (7-36amide) in GLP-1R K0 mice [201]. The superior cardioprotective effect of GLP-1 (7-36amide) relative to exendin- 4 and the preservation of efficacy of GLP-1 (7-36amide) but not exendin-4 in GLP-1R K0 mice suggest that some of the cardioprotective effects of GLP-1 (7-36amide) are independent of GLP-1R signaling and are potentially mediated via the DPP-4-cleaved GLP-1 metabolite GLP-1 (9-36amide) [201]. Interestingly, when mice are pretreated with either GLP-1 (7-36amide) or GLP-1 (9-36amide), only GLP-1 (7-36amide) improves LVDP. However, when administered during reperfusion, both GLP-1 (7-36amide) and GLP-1 (9-36amide) improve functional recovery from I/R injury in both WT and GLP-1R K0 mice [201]. Consistent with these data, while exendin-4 but not GLP-1 $(9-36)$ has infarct limiting potential in the isolated rat heart, exendin-4 and GLP-1 (9-36) both improve left ventricular performance during reperfusion [626]. The infarct-limiting potential of exendin-4 is abolished by treatment with exendin $(9-39)$, thus indicating that this cardioprotective effect is GLP-1R-dependent [626]. Treatment of isolated mouse cardiomyocytes with either exendin4 or GLP-1 (936amide) increases phosphorylation of AKT and ERK [631], both of which are known to positively affect cardiomyocyte growth and survival $[632,633]$.

Collectively, these data indicate that pharmacologic administration of GLP-1 (9-36amide) may have a functional role to improve recovery from I/R injury via GLP-1R-independent mechanisms, whereas GLP-1 (7-36amide) but not GLP-1 (9-36amide) affects cardiac contractility via GLP-1R signaling [201]. Further supporting a cardiometabolic role of GLP-1 (9-36amide), both GLP-1 (7-36amide) and GLP-1 (9-36amide), but not exendin-4, promote vasodilation in isolated and ex vivo cultured mesenteric blood vessels [201], i.e., the vasodilatory effect of GLP-1 (7-36amide) was not different relative to that of GLP-1 (9-36amide) and was likewise preserved in GLP-1R KO mice [201]. Whether the low endogenous concentration and the restricted pharmacokinetic action profile of endogenous GLP-1 metabolites are sufficient to elicit such effects under physiological circumstances remains questionable.

12.3. Effect of GLP-1 on heart rate (HR) and blood pressure (BP) GLP-1 effects on HR and BP are species-related. In rats, intravenous infusion of GLP-1 (7-37amide) or exendin-4 acutely increases HR as well as systolic, diastolic, and mean arterial BP (Figure 7) [634,635]. In rats, GLP-1 stimulation of BP peaks around 3-5 min post-injection and returns to baseline after $25 \mathrm{~min}$ [634]. Both central and peripheral administration of GLP-1R agonists increase HR and BP in rodents [636-638], with induction of c-Fos expression in adrenal medullary catecholamine neurons and activation of tyrosine hydroxylase, the key enzyme involved in production of norepinephrine, in the brainstem [638]. These data collectively suggest that some of the acute cardiovascular effects of GLP-1 might be due to brain enhancement of catecholamine outflow and elevation of sympathetic tone. However, in rats, GLP-1 elevation of BP is preserved upon pretreatment with reserpine, propanolol or phentolamine, thus indicating that the hypertensive effect of GLP-1 does not exclusively depend on catecholamine signaling [634]. However, while the hypertensive effect of GLP-1 is not abolished by inhibition of $\beta$-adrenergic signaling [634], exendin4 induction of HR is blocked by the $\beta$-adrenergic receptor antagonist propranolol [639], and exendin-4 fails to affect HR in adrenalectomized rats (as reviewed in [622]). In mice, both acute and chronic central administration of exendin-4 increases HR and BP with concomitant decrease in excitatory glutamatergic and inhibitory glycinergic neurotransmission to preganglionic parasympathetic cardiac vagal neurons. GLP-1 stimulation of $\mathrm{HR}$ and BP is mediated via GLP-1R signaling because i.v. infusion of exendin (9-39) blocks GLP-1 and exendin-4 induction of HR and BP [635], and these actions are lost in Glp $1 r^{-/-}$mice [640]. Collectively, these data suggest that acute GLP-1 effects on HR and BP in normal animals may involve signaling via both, the sympathetic and parasympathetic nervous systems. Not all rodent studies report elevated BP in response to GLP-1R agonism, but dose could be a discriminatory factor. While some studies report increased BP in rodents upon acute or chronic GLP-1R agonism [634-636,638], others report decreased $\mathrm{BP}$, especially in experimental models associated with the development of hypertension such as salt-sensitive $\mathrm{db} /$ 
db mice and Dahl S rats $[641,642]$. In mice, liraglutide blunts hypertension induced by angiotensin II (Ang-II), an effect that is absent in GLP-1R K0 mice, and is also attenuated upon pretreatment of WT mice with exendin (9-39) [640].

While in most rodent studies GLP-1 increases HR and BP, i.v. infusion of GLP-1 (7-36amide) acutely increases HR without affecting mean arterial BP in conscious calves [643]. Most human studies report a moderate stimulatory effect of GLP-1 on HR with unchanged or with reduced BP only in hypertensive individuals [620,622,640,644-649]. Acute infusion of GLP-1 increases heart rate and cardiac output in healthy human volunteers, potentially via GLP-1-induced vasodilation in adipose tissue and skeletal muscle [650]. As demonstrated in healthy human volunteers, GLP-1 infusion acutely increases blood flow in the subcutaneous abdominal adipose tissue and skeletal muscle without alterations in splanchnic blood flow [650]. Notably, in contrast to GIP [651-654], the GLP-1 induced increase in adipose tissue blood flow is not dependent on postprandial hyperglycemia and hyperinsulinemia and is observed even under conditions of fasting glucose and insulin concentrations [650].

GLP-1R agonism has also been demonstrated to reduce BP in people with T2DM (as reviewed in $[611,622,649])$. Notably, in healthy human volunteers, acute administration of GLP-1, GLP-1 (9-36amide), or exenatide did not affect blood flow in the mesenteric or renal arteries, excluding splanchnic vasodilatation as a potential mechanism underlying the chronic hypotensive effect of GLP-1 [644]. In a recent metaanalysis of 60 clinical studies, GLP-1R agonists were demonstrated to reduce diastolic BP with a range of -1.84 to $-4.60 \mathrm{mmHg}$ and to slightly increase heart rate by $2-3.35$ beats/min [620]. Relative to placebo, the reduction of diastolic BP only reached significance for the treatment of exenatide $10 \mu \mathrm{g} /$ twice daily $(-1.08 \mathrm{mmHg})$ [620]. These data align with an integrated analysis of 36 clinical studies that affirmed cardiovascular safety with up to 4-years' treatment with either DPP-4 inhibitors or GLP-1R agonists [655].

The molecular mechanisms underlying GLP-1 improvement of BP are incompletely understood. Potential mechanisms may include GLP-1induced vasodilation and activation of nitric-oxide-dependent mechanisms [612,640,656]. Indeed, liraglutide stimulates relaxation of the aorta [640] but GLP-1 might also indirectly lower BP via its ability to reduce body weight. GLP-1R agonism could also improve BP via its ability to stimulate sodium excretion (natriuresis) via the kidneys [657]. The long-term steady-state BP is affected by the intravascular volume, which is influenced by the vascular tone and the extracellular fluid volume (ECFV); the ECFV is in turn determined by the sodium balance. Accordingly, when BP increases, the kidneys respond by increasing natriuresis to decrease $B P$ via reduction of the ECFV $[92,658,659]$. GLP-1R agonists acutely stimulate natriuresis in rodents and may also do so in humans, lowering $B P$, potentially also via reduction of the ECFV [641,642,660,661]. In mice, liraglutide reduces angiotensin II (Ang-II) stimulation of systolic and diastolic BP, an effect that is absent GLP-1R KO mice or by pretreatment with exendin (9-39) or the natriuretic peptide receptor antagonist anantin [640]. In WT mice and in isolated atrial cardiomyocytes, liraglutide stimulates the production and secretion of atrial natriuretic peptide (ANP), and liraglutide fails to induce natriuresis and to lower BP in ANP KO mice [640]. Liraglutide stimulation of cardiac ANP secretion is PKA independent but mediated by Epac2 in a cAMP and PLCdependent manner [640]. However, in humans there is no consistent effect of GLP-1 on ANP secretion whereas angiotensin II is generally lowered [657,662-664]. In summary, GLP-1R agonism improves BP through stimulation of natriuresis via Epac2-dependent stimulation of cardiac ANP secretion [640]. Nevertheless, the majority of hypertensive human subjects treated with liraglutide do not exhibit an increase in circulating ANP [662].

\section{GLP-1 EFFECTS ON FOOD INTAKE AND BODY WEIGHT}

One of the most frequently described extrapancreatic effects of pharmacokinetically-optimized GLP-1R analogs is their ability to lower body weight via centrally regulated inhibition of food intake (Figure 7). Suppression of food intake by GLP-1R agonism has been demonstrated in numerous species including mice [465-467,665,666], rats $[667,668]$, birds $[669,670]$, pigs [671,672], non-human primates [673,674], and humans [675-678]. The anorexigenic effect of GLP-1R agonism has been demonstrated in numerous clinical studies and fMRI analysis support that GLP-1 inhibition of food intake is centrally mediated in healthy individuals and patients with type-2 diabetes [679-684].

\subsection{Effect of peripheral administration of GLP-1 analogs on food intake}

Peripheral administration of GLP-1 analogs decreases body weight via suppression of food intake [465-467,665,685]. Depending on the molecule (native GLP-1 or a longer-acting GLP-1 analog) and the route of administration, GLP-1 and its analogs seem to engage different signaling pathways to either directly or indirectly transmit the signal to reduce food intake to the CNS.

Central GLP-1R appear to be necessary for the anorectic effect of peripherally administered GLP-1R agonists in rodents [686-688]. When administered s.c., liraglutide fails to inhibit eating in mice possessing a CNS-specific deletion of GLP-1R [688]. Another report indicates that i.v. infusion of liraglutide inhibits eating in rats by directly activating hypothalamic Pomc/Cart neurons in the ARC, without activating GLP-1-producing neurons in the hindbrain, and independent of GLP-1R signaling in vagal afferents, the area postrema (AP) and the PVN [686]. In rats, central (i.c.v.) administration of exendin (9-39) was also sufficient to attenuate the anorexigenic effect of i.p. injected liraglutide and exendin-4 [687], and bilateral neurochemical lesions of the lateral parabrachial nucleus (IPBN) with ibotenic acid blunted the anorexigenic effect of peripherally administered exendin-4 [689]. When applied either s.c. or i.p., exendin-4 and liraglutide induced acute neuronal activation (measured by activation of cFos) in PVN, AP, and NTS [686,690]. Interestingly, the short-term anorexigenic effects of i.p. injected low-dose liraglutide and exendin- 4 were also attenuated by subdiaphragmatic vagal deafferentation $[687,691]$, but longer-term effects of these compounds on food intake did not depend on intact vagal afferents [691]. All of these findings are consistent with the concept that GLP-1 receptor agonists inhibit eating and reduce body weight primarily by acting directly in the brain, and that various brain areas are involved in mediating the eating-inhibitory effects of GLP-1 receptor agonists. This presumably reflects the ability of long-acting GLP-1 analogs to enter the brain at areas with an incomplete BBB, such as the median eminence.

While these data collectively emphasize the importance of neuronal GLP-1R for the food intake inhibition by long-acting GLP-1 analogs, it is unclear whether and to what extend this accounts for physiological doses of intestinal-derived native GLP-1. When infused into the hepatic portal vein, GLP-1 (7-36amide) increased cFos expression in the NTS, the AP, and the central nucleus of the amygdala, but not in the hypothalamic ARC or PVN [692], i.e., native GLP-1 appears to induce an activation pattern in the brain that is different from the pattern induced by long-acting GLP-1 receptor agonists. While peripherally administered GLP-1 seemingly crosses the blood brain barrier (BBB) via simple 
diffusion, the half-life of GLP-1 is less than 2 min, leading to disagreement regarding whether peripherally derived GLP-1 is what activates CNS GLP-1R under physiological conditions.

In rats, i.v. administration of GLP-1 at high physiological doses stimulates vagal afferents, and this is blocked by pretreatment with exendin (9-39) [693]. While this activation appears to be involved in the insulinotropic and gastric emptying inhibiting effects of exogenous GLP-1 [693], it does not appear to be crucial for the effect of i.v. administered native GLP-1 on eating [694], because GLP-1 infusions into the hepatic portal vein did not lose their potency to reduce food intake after subdiaphragmatic vagal deafferentation in rats [692,694]. Interestingly, however, the food intake inhibition following i.p. administration of a supposedly "physiological" dose of GLP-1 (736amide) depends on the integrity of abdominal vagal afferents [694], indicating that peripheral GLP-1 can inhibit eating via at least two partly separate pathways. Consistent with a role of vagal afferents and vagal afferent GLP-1 receptors in the eating-inhibitory effect of peripheral endogenous GLP-1, virus-mediated knockdown of GLP-1R in vagal afferent neurons increased meal size, accelerated gastric emptying, and reduced prandial insulin secretion in rats, while not affecting $24 \mathrm{~h}$ food intake or body weight [518]. The latter finding matches the results obtained in Phox2b-Cre GLP-1 receptor K0 mice, lacking GLP-1 receptors in the autonomic nervous system, which have normal body mass and composition in response to both chow and HFD [688], yet exhibit partial attenuation of the anorectic and weight loss effects of peripherally administered GLP-1R agonists [520]. In humans, the effect of i.v. GLP-1 infusion on food intake was lost after truncal vagotomy [695], indicating that vagal afferents are involved in mediating the effects of circulating native GLP-1 on appetite. In rats, on the other hand, lesions of the AP or administration of exendin (9-39) into the 4th ventricle blocked the food intake inhibition by GLP-1 infused into the hepatic portal vein [261], suggesting that high concentrations of circulating GLP-1 act on the hindbrain to reduce food intake. In one study GLP-1 infused into the vena cava or hepatic portal vein of rats had similar effects on food intake [696], whereas in another study in rats GLP-1 infusions into the jugular vein inhibited food intake more potently than infusions into the hepatic portal vein [697]. These findings argue against a hepatic portal site of action of GLP-1 to inhibit eating, and further emphasize that the route of administration is an important consideration when analyzing food intake inhibition by GLP$1 \mathrm{R}$ agonism.

The data summarized above were collected in mice, rats, and humans; in addition to varying experimental conditions, species differences may account for some of the discrepancies observed. Nevertheless, collectively these data indicate that vagal afferents are involved in mediating the satiating effects of endogenous peripheral GLP-1 or exogenously administered GLP-1 and the acute inhibition of food intake in response to peripheral GLP-1 receptor agonists under certain conditions. However, vagal afferents and vagal afferent GLP-1 receptors are presumably less important for the effects of peripheral GLP-1 on body mass.

\subsection{Effect of central administration of GLP-1R analogs on food} intake

The acute anorectic effect of centrally administered GLP-1 was described simultaneously 1996 by Steve Bloom's group [668] and by Mads Tang-Christensen et al. [92] and was confirmed by others $[667,698]$. In mice and rats, the short-term inhibition of food intake induced by i.c.v. administered GLP-1R agonists is dose-dependent, can be blocked by pretreatment with exendin $(9-39)[668,698]$ and is absent in GLP-1R K0 mice [690]. Inhibition of food intake by centrally administered GLP-1R analogs is accompanied by increased cFos neuronal activity in the PVN, amygdala, NTS, AP, lateral parabrachial nucleus, and the ARC $[668,696,698]$. These data align with the expression of GLP-1R throughout the CNS [395,396]. Furthermore, cell bodies that are immunoreactive to GLP-1 antisera are detected in the NTS, and GLP-1 immunoreactive nerve fibers are widely distributed throughout the brain with highest density in the hypothalamus, thalamus and septal regions whereas the lowest density is in the cortex and hindbrain $[86,698]$. Supporting a role of the NTS in mediating GLP1 's effects on food intake, adeno-associated virus (AAV) mediated knock-down of GLP-1R in the NTS results in higher food intake and meal size [699]. Chronic blockade of GLP-1R with intra 3rd ventricular infusion of exendin (9-39) increases food intake and fat mass [700]. Direct intraparenchymal delivery of low, ventricle subthreshold doses of GLP1R agonists to GLP1R expressing nuclei throughout the brain, including e.g., NTS, IPBN, ventral tegmental area (VTA), PVH, PVT, nucleus accumbens (NAcc), ventral hippocampus, and lateral septum reduced food intake via reductions in meal size. Consistent with this, delivery of exendin (9-39) to these sites increases meal size and food intake [701-705].

Hindbrain GLP-1R activation enhances phosphorylation of PKA and MAPK while decreasing the activity of AMPK in the NTS, and inhibition of PKA/MAPK activity by administration of Rp-cAMP or U0126 attenuates food intake inhibition of exendin-4 administered into the 4th ventricle [706]. These data suggest that hindbrain GLP-1R activation suppresses food intake via PKA/MAPK-induced inhibition of AMPK. Diphtheria toxin-induced ablation of GLP-1 producing neurons in the NTS had no effect on either ad libitum food intake or glucose metabolism [90]. Nevertheless, in rats, activation of hindbrain GLP-1R via i.c.v. administration of exendin-4 into the 4th ventricle reduces food intake via reduction of meal frequency; i.e., via increased satiety [706]. Intra 4th-ventricular administration of exendin (9-39) or lesions of the AP also block food intake inhibition by GLP-1 infused into the hepatic portal vein (HPV) [261], suggesting that high levels of circulating GLP-1 inhibit eating at least in part by acting on the hindbrain.

\subsection{Molecular mechanisms underlying food intake inhibition by GLP-1}

The molecular underpinnings regulating GLP-1 inhibition of food intake and body weight loss are complex. Undoubtedly, brain GLP-1R signaling is crucial for the anorexigenic effect of exogenously administered long-acting GLP-1R agonists, as demonstrated by failure of liraglutide to suppress food intake in CNS-specific GLP-1R KO mice [688]. Consistent with this, i.c.v. administration of exendin (9-39) is sufficient to attenuate the anorexigenic effect of peripherally administered liraglutide and exendin-4 [687]. However, CNS-specific GLP-1R $\mathrm{KO}$ mice have normal body mass and composition whether on a chow or HFD [688], suggesting that these receptors may be necessary for the pharmacological response to GLP-1 but not the physiological control of body weight. However, data obtained in $\mathrm{KO}$ animals generally have to be regarded with caution since compensatory mechanisms, which limit interpretations and affect study outcomes, might develop. Depending on the route of administration (and the GLP-1R agonist used), peripherally administered GLP-1 analogs seem either to act directly in the hypothalamus and hindbrain, both areas with incomplete $\mathrm{BBB}$, or else on vagal afferents to transmit the signal to the hindbrain which then projects to other key feeding areas in the brain. Hindbrain GLP-1R activation enhances phosphorylation of PKA and MAPK while decreasing the activity of AMPK in the NTS, and inhibition of PKA/MAPK activity by administration of Rp-cAMP or U0126 attenuates food intake inhibition of exendin-4 administered into the 4 th ventricle [706]. These 
data suggest that hindbrain GLP-1R activation suppresses food intake via PKA/MAPK-induced inhibition of AMPK.

The anorexigenic effect of GLP-1R agonism involves the glucosedependent silencing of central AMPK action. Activity of AMPK is increased under conditions of fasting [707] and is decreased under conditions of enhanced glucose utilization [708-710]. Consistent with AMPK's role as a stress- and nutrient-responsive protein kinase, pharmacological, physiological (e.g. fasting-induced), or genetic activation of AMPK stimulates food intake whereas AMPK inhibition decreases food consumption [707,711]. Central administration of GLP-1 reduces the activity (phosphorylation) of AMPK [712,713] and treatment of mouse hypothalamic GT1-7 cells with exendin-4 stimulates glycolysis and decreases phosphorylation of AMPK [714]. Pharmacological activation of AMPK by administration of 5-aminoimidazole-4carboxamide-1- $\beta$-D-ribofuranoside (AICAR) in either the 3rd [714] or the 4th ventricle [706] attenuates the anorectic effect of exendin-4 injected into the same ventricles. Consistent with these data, i.c.v. administration of the glycolysis inhibitor 2-deoxyglucose (2-DG) likewise increases activity of AMPK and blunts food intake suppression by i.c.v.-injected exendin-4 [714]. In summary, compelling evidence indicates that the anorexigenic effect of central GLP-1 receptor activation is partially mediated by central stimulation of glycolysis and concomitant silencing of AMPK activity. Accordingly, pharmacological activation of AMPK in the VMH blunts food intake suppression by i.c.v. exendin-4 [714,715] and attenuates central GLP-1 effects on brown and inguinal white adipose tissue [666].

The anorexigenic effect of GLP-1 seems to be transduced via GLP-1R signaling in several areas of the brain, including both hypothalamic nuclei and the hindbrain. Microinjection of exendin-4 into the NTS reduces intake of palatable high-fat diet and inhibits food reward behavior [716], and hindbrain inhibition of GLP-1R via administration of exendin (9-39) into the 4th ventricle increases food intake [717]. The anorexigenic and weight-lowering effects of liraglutide are blunted upon genetic ablation of GLP-1R in glutamatergic neurons, yet fully preserved when GLP-1R is deleted in GABAergic neurons [718]. Peripheral administration of GLP-1 (7-36amide) enhances neuronal activity in the VMH $[719,720]$, and direct administration of GLP-1 into the LH, VMH, or DMH of rats acutely decreases food intake [721]. Similar findings were reported in rats that received direct infusion of GLP-1 (736amide) into the PVN [722,723]. In rats, liraglutide decreases food intake when directly injected into the ARC, LHA, or PVN while direct administration into the $\mathrm{VMH}$ decreases body weight without affecting food intake, but increases the expression of Ucp1 in the brown and inguinal white adipose tissue [666]. The acute effects of exendin-4 to reduce food intake and to improve glucose handling are preserved upon knockdown of GLP-1R in the VMH [715], indicating that the effects of central GLP-1R activation on eating and BAT thermogenesis are mediated by at least partially separate brain areas and circuitries.

\subsection{GLP-1 effects on visceral illness}

Inhibition of food intake can be due to other factors than satiety, including gastric discomfort, e.g., gastroparesis, associated with visceral illness and stress. Anorexia associated with visceral illness can be chemically-induced by administration of lithium chloride (LiCl), a substance known to promote nausea and emesis in humans [724726] and taste avoidance conditioning and pica in rodents, that do not vomit [727]. Pica is the measure of the amount of kaolin clay consumed in response to treatments that trigger nausea and vomiting in humans and is a broadly accepted proxy of visceral malaise in nonvomiting rats. Taste avoidance conditioning describes the reduction in the intake of novel taste or food that has been associated with a treatment that induces negative consequences e.g., including the effects of peripheral pain, lactose malabsorption or the effects $\mathrm{LiCl}$ [728]. In rats, peripheral administration of $\mathrm{LiCl}$ induces cFos neuronal activity in the IPBN, the AP and the NTS [726,729] with remarkable overlap in the cFos activity pattern induced by centrally administered GLP-1 [726]. Apart from inducing cFos activity in the same brain areas [696,726,730], LiCl and central GLP-1R agonism both decrease Iocomotor activity [668,731], slow Gl motility and gastric emptying [573,732], reduce body temperature [732,733], and induce adverse gastrointestinal effects including nausea, emesis, and taste avoidance [696,724-726,734-737]. Selective ablation of NTS PPG neurons prevents stress-induced hyperphagia in mice but has no effect on long-term ad libitum food intake, body weight, or glucose tolerance [90]. These data suggest that NTS PPG neurons are not essential for long-term regulation of food intake but are required for the short-term hypophagia in response to stress $[738,739]$.

Collectively, these studies highlight that, in some species, GLP-1 and $\mathrm{LiCl}$ might engage similar central signaling mechanisms and suggest that central GLP-1s anorexigenic effect might be influenced by aversive pathways and gastric discomfort. However, there are other data that would challenge the simple explanation that the reduction in feeding and body weight resulting from CNS GLP1R agonism is simply explained by inducing an aversive visceral state. Targeted agonist delivery to CNS GLP1-R expressing sites, PVT, NTS, VTA, NAcc, or PBN reduces food intake without the expression of pica, a proxy of visceral illness [704,716,740,741]. In addition, Jelsing et al. report that food intake inhibition by liraglutide is observed in the absence of reduced gastric emptying effects that some have linked to visceral malaise [742]. Importantly, both van Bloomendaal et al. [682] and ten Kulve et al. [743] provide data to support the hypothesis that food intake reduction after GLP-1 receptor agonism is mediated by actions on appetite- and reward-related areas of the human brain, an interpretation also supported by rodent studies $[704,716,740,741]$.

\subsection{GLP-1 effects on reward behavior}

Central GLP-1 regulation of food intake is not restricted to GLP-1R signaling in the hypothalamus and hindbrain and is not limited to modulation of homeostatic feeding, i.e. food intake to maintain energy homeostasis. GLP-1 also affects non-hunger related (hedonic) feeding by targeting brain areas involved in reward, motivation and addiction, such as the VTA, NAcc, lateral septum [701,741,744,745], and PVT [704]. These mesolimbic regions express GLP-1R [347] and receive projections from PPG neurons in the NTS $[354,741,744]$. Peripheral administration of exendin-4 [691] or direct administration of GLP-1 into the core of the NAcc [744] increases cFos expression in this region, and direct activation of NAcc GLP-1R reduces food intake while its direct inhibition by exendin (9-39) induces hyperphagia [744]. Food intake inhibition by NAcc GLP-1R is unrelated to taste avoidance [744] and does not induce pica [701,741]. Administration of exendin-4 into the shell of the NAcc decreases sucrose reward behavior in the progressive ratio (PR) operant conditioning test [701]. Consistent with this, GLP-1R agonism decreases a variety of reward behaviors in rodents, including the motivation to lever press for food reward in an operant conditioning test [746], alcohol intake [747,748], alcohol seeking behavior [747], amphetamine-induced conditioned place preference (Amp-CPP) [748], and hedonic feeding [748]. The inhibitory effect of GLP-1R analogs on reward behaviors is blunted in mice with CNSspecific deletion of GLP-1R [748]. Several lines of evidence indicate that NAcc GLP-1R activation decreases food intake by reducing food palatability. In satiated rats given a choice between chow and high-fat diet (HFD), exendin-4 preferentially reduces consumption of the more 
palatable HFD [741]. In rats, intra-NAcc injection of exendin (9-39) increases the size of a sucrose meal and accelerates the licking frequency during the first meal, an indicator of increased palatability [749]. In summary, GLP-1R agonism decreases homeostatic and hedonic feeding, and modulates food intake not only via the hypothalamus and the hindbrain but also via signaling in the mesolimbic system, in which GLP-1R activation affects reward behavior and palatability.

\subsection{GLP-1 transport across the blood brain barrier (BBB)}

Several lines of evidence indicate that peripherally administered GLP-1 can cross the blood brain barrier (BBB). Studies using a stabilized radiolabeled (Ser8)GLP-1 revealed rapid uptake into the brain after intravenous (i.v.) administration without self-inhibition when challenged with increased doses of unlabeled (Ser8)GLP-1 or upon inhibition of brain GLP-1 influx upon pretreatment with exendin (9-39). Small peptide GLP-1R analogs including liraglutide, lixisenatide, and exendin-4 have all been demonstrated to cross the BBB upon peripheral administration [750,751], and HPLC analysis confirmed that most of the peripherally administered exendin- 4 reaches the brain intact $[751,752]$. While these studies suggest that GLP-1 influx into the brain might be a GLP-1R-independent process with passive diffusion of GLP-1 through the BBB [750-752], other studies suggest that uptake of liraglutide into the brain is GLP-1R-dependent [686,753]. Nonetheless, brain regions with incomplete BBB, such as the subfornical region and the $A P$, are main targets of peripherally injected 125Ilabeled GLP-1 [754].

\section{GLP-1 EFFECTS ON WATER INTAKE}

GLP-1 inhibition of water intake was first reported by Mads TangChristensen in 1996 [92] and was later confirmed by others [755760]. GLP-1 inhibition of water consumption is independent of food intake [758] and is blocked by pretreatment with exendin (9-39) [92]. In rats, inhibition of drinking by GLP-1 (7-36 amide) is dosedependent and occurs within 30 min following its central (i.c.v.) or peripheral (i.p.) administration [92]. In one study in healthy humans, i.v. infusion of GLP-1 decreased water consumption by $36 \%$ in response to i.v. infusion of hypertonic saline $(5 \% \mathrm{NaCl})$ [755]. Peripheral but not central administration of GLP-1 fails to inhibit angiotensin (ANG) II-induced drinking in rats that have been treated with monosodium glutamate (MSG) to induce damage of the ARC and the sensory circumventricular organs, suggesting that peripherally administered GLP-1 effects drinking behavior via CNS-dependent mechanisms [760].

GLP-1 affects renal function by decreasing water intake as well as by stimulating the excretion of urine and sodium (Figure 7). The natriuretic and diuretic effect of GLP-1R agonism was first shown in 1996 [92] and was subsequently confirmed in rodents [642,660,761] and in healthy, obese, and type-2 diabetic humans $[661,663]$. While the acute natriuretic effect of GLP-1 has been solidly confirmed in humans [762-764], 12 wk treatment of T2DM patients with liraglutide or the DPP-4 inhibitor sitagliptin had no effect on markers of renal function, including glomerular filtration rate, renal plasma flow, albumin-tocreatine ratio, and excretion of sodium, potassium, and urea [765]. These data suggest that that GLP-1's effects on renal function might be transient and vanish upon more chronic treatment. In one study in T2DM patients, the natriuretic effect of liraglutide persisted after 3 wk treatment [662].

In rats, GLP-1 induced natriuresis and diuresis is accompanied by increased renal plasma flow and glomerular filtration rate [660,761]. Denervation of the kidney results in failure of GLP-1 to increase glomerular filtration rate and decreases the natriuretic and diuretic effect of GLP-1 [761]. These data suggest that the natriuretic and diuretic effect of GLP-1 might be due to inhibition of sodium reabsorption in the proximal tubule and emphasize that GLP-1 requires intact renal innervation to increase glomerular filtration. Studies in rodents and humans indicate that GLP-1R is expressed in the renal vasculature [392,766-768]. However, treatment of isolated porcine proximal tubular cells with GLP-1 reduced sodium re-absorption [768]. Consistent with this, GLP-1, exendin-4, and the DPP-4 inhibitor sitagliptin reduced proximal tubular sodium reabsorption in rats $[660,761,769,770]$ and humans [663]. In the renal proximal tubule, GLP-1 reduced $\mathrm{Na}^{+} / \mathrm{H}^{+}$exchanger isoform 3 (NHE3)-mediated bicarbonate reabsorption [660]. Consistent with this, exendin-4 decreased NHE3-mediated sodium-dependent $\mathrm{pH}$ recovery in porcine kidney LLC-PK 1 cells [771]. Inhibition of NHE3 activity by GLP$1 \mathrm{R}$ agonism seems to be mediated via CAMP activation of PKA, which phosphorylates NHE3 at its Ser552 and Ser605 residues [660,771,772]. In rodents [660,761] and humans [663,763], GLP-1R agonism increases renal lithium clearance, a marker for proximal tubular sodium reabsorption. These data suggest that the diuretic and natriuretic effects of GLP-1 include changes in renal hemodynamics and reduced sodium reabsorption due to PKA-dependent downregulation of NHE3 activity in the renal proximal tubule. GLP-1 might also affect kidney function via its cardiovascular effects. In rats, infusion of GLP-1 increases renal blood flow and the glomerular filtration rate $[660,761]$, and this effect is potentially mediated via GLP1 's ability to increase vasodilation of the afferent renal arteriole. In wildtype but not GLP-1R KO mice, liraglutide stimulates the secretion of natriuretic peptide (ANP) from the atrial cardiomyocytes and liraglutide fails to affect natriuresis and vasorelaxation in mice deficient for ANP [640].

\section{GLP-1 EFFECTS ON ENERGY EXPENDITURE}

A role of endogenous GLP-1R signaling in the control of energy expenditure was inferred from studying mice with congenital GLP-1R deficiency. Relative to wildtype controls, lean chow-fed GLP-1R KO mice do not have alterations in energy expenditure, neither when kept at a standard room temperature nor when challenged with moderate cold exposure [773]. However, relative to wildtype controls, dietinduced obese GLP-1R KO mice exhibit reduced energy expenditure at standard ambient room temperature but not at temperatures favoring thermoneutrality [774]. Because thermoneutrality minimizes the contribution of non-shivering thermogenesis to whole-body energy expenditure and because non-shivering thermogenesis requires functional brown adipose tissue (BAT) [775], these data imply a contribution of endogenous GLP-1R signaling to the control of energy expenditure, at least in diet-induced obese mice, by regulating BAT activity. This contribution requires GLP-1R signaling in the brain; i.e., the brain plays a critical role in the control of BAT activity [776] and GLP-1R is expressed in the brain, including in nuclei involved in the control of BAT $[347,389]$. In keeping with this, site-specific disruption of GLP-1R on neurons of the DMH of rats results in significant body weight gain paralleled by a reduction in energy expenditure and BAT heat production [777]. The DMH is part of the neuronal network involved in the control of BAT thermogenesis [776] and receives innervation from pre-proglucagon-expressing neurons of the hindbrain [778]. A priori, this positions those neurons as potential contributors to the neural circuits controlling energy expenditure by regulating BAT thermogenesis in rodents. Intriguingly, emerging data involving gain or loss of function from those cells in mice argue against such a 
contribution. Thus, diphtheria toxin-mediated ablation of those neurons [90] does not result in long-term changes on food intake or body weight, which suggests a minimal impact on energy expenditure. Conversely, acute chemogenetic activation of those neurons fails to increase, and rather transiently reduces, energy expenditure [357]. Disregarding differences due to experimental conditions including acute versus chronic intervention or animal species or strains, these data suggest that endogenous GLP-1R signaling in the brain may play a constitutive role in the homeostatic control of BAT thermogenesis, beyond its physiological modulation by brain-derived GLP-1.

However, this does not preclude the possibility that exogenous administration of GLP-1 may regulate energy expenditure by acting on brain GLP-1R. In fact, an early report in rats described an increase in energy expenditure during the first hours of the dark cycle following i.c.v. administration of low doses of GLP-1 (7-37amide) [779].

In mice, acute central (i.c.v.) but not peripheral administration of GLP-1 increased brown adipose tissue (BAT) temperature and increased the activity of sympathetic fibers that innervate the inguinal BAT [773]. Consistent with these data, central administration of the GLP-1R agonists liraglutide and exendin-4 increases energy expenditure and BAT thermogenesis through mechanisms that include AMPK signaling in the mediobasal hypothalamus [666]. Taken together, these studies suggest that pharmacological activation of brain GLP-1R signaling increases energy expenditure in mice.

Peripheral administration of GLP-1R agonists has led to variable results with respect to energy expenditure. One study in rats found an increase of energy expenditure upon i.v. infusion of GLP-1, and with even greater potency relative to centrally administered GLP-1 [780]. These data are consistent with the demonstration that i.v. but not i.p. administered GLP-1 inhibits eating via activation of hindbrain GLP-1 receptors $[261,694]$ and suggests a similar mechanism for i.v. GLP-1-stimulation of energy expenditure. In contrast, another study in rats reported an acute decrease of energy expenditure associated with a decrease in interscapular skin temperature following i.p. administration of exendin-4 and this effect was attenuated when GLP-1R was knocked down on vagal afferent neurons [781]. These data are consistent with reports that the anorexigenic effect of i.p. administered GLP-1 depends on vagal afferents [692,694] and thus suggests a similar mechanism for i.p. GLP-1 effects on energy expenditure. Caudal brainstem delivery of Cocaine- and amphetamine-regulated transcript (CART) induces hypothermia, and this effect is blocked by pretreatment with exendin (9-39) [782]. Several studies in mice [465,467,685,774] and humans [783-787] have failed to detect changes in energy expenditure following chronic or acute peripheral treatment with different GLP-1R agonists. One human study did report an acute decrease of diet-induced thermogenesis due to lower carbohydrate oxidation following i.v. infusion of GLP-1 [788].

In summary, the experimental evidence supports a contribution of brain-GLP-1R signaling in the control of energy expenditure, at least in rodents, via control of BAT activity. The frequently reported absence of peripherally administered GLP-1 mimetics to regulate energy expenditure in mice and humans has several implications. First, the BBB might limit access to brain areas where GLP-1R could potentially mediate effects on energy expenditure; second, the underlying GLP-1 analog, the feeding status of the test subject, and the route of administration are important considerations when analyzing GLP-1 effects on energy expenditure, and, third, rodents may be uniquely sensitive to mechanisms activating energy expenditure through the sympathetic nervous system.

\section{GLP-1 EFFECTS ON GASTRIC EMPTYING}

In addition to its direct and vagally mediated insulinotropic action on $\beta$ cells, GLP-1 also improves postprandial glucose handling by inhibition of gastric emptying, decelerating the rate at which glucose is absorbed into the circulation (Figure 7) [573,789-791]. Inhibition of gastric motility and gastric acid secretion following treatment with GLP-1R agonists has been demonstrated in mice [792], rats [793-795], dogs [796-798], pigs [799,800], and humans [573,789$791,799,801-805]$. In rats, inhibition of gastric emptying occurs after peripheral or central administration of GLP-1 [795], and this effect is mediated via hindbrain GLP-1R signaling [806]. Vagal afferent GLP-1 receptor knockdown accelerates gastric emptying [518] and vagal afferent denervation [795] or peripheral administration of exendin (939) $[794,795]$ blocks the effect of centrally or peripherally administered GLP-1 on gastric emptying. These data are consistent with the failure of GLP-1 to inhibit gastric acid secretion in vagotomized human subjects [807] as well as the failure of GLP-1 to inhibit vagally-induced motility in the isolated perfused pig antrum [800]. These data suggest that GLP-1-mediated inhibition of gastric motility includes vagal afferents, as well as GLP-1R dependency in the periphery and the brain [795]. Inhibition of adrenergic signaling through combined administration of phentolamine and propanolol abolishes the inhibitory effect of GLP-1 on gastric motility, suggesting that GLP-1 inhibits gastric emptying via adrenergic signaling [794]. In fasted rats, coadministration of GLP-1 and GLP-2 leads to a greater inhibition of small bowel motility relative to either monotherapy [793]. Exendin-4 fails to affect gastric emptying in GLP-1R KO mice [792]. In healthy human volunteers, intravenous GLP-1 decelerates gastric emptying after the first meal to a greater extent than after a second meal, indicating that GLP-1 inhibition of gastric emptying undergoes rapid desensitization [803]. In overweight humans, liraglutide $3 \mathrm{mg}$ solidly inhibits gastric emptying after 5wk of treatment, but the efficacy markedly decreases after 16 wk of treatment, yet with still significant effect relative to placebo treated controls [808]. In rats, liraglutide- but not exendin-4-induced inhibition of gastric emptying is markedly diminished after $14 \mathrm{wk}$ of treatment [742]. Because gastric emptying is a prerequisite for glycemic rises after meals, which in turn trigger insulin secretion, deceleration of gastric emptying in response to exogenous GLP-1 leads to lesser post-meal glucose excursions and reduced insulin secretory responses [809]. This has led to doubts about the importance of an incretin role for pharmacological GLP-1 administration, because incretin hormones should lead to a net increase in post-nutrient insulin secretory responses [810].

\section{GLP-1 EFFECTS ON THE HYPOTHALAMIC PITUITARY ADRENAL (HPA) AXIS}

Neurons of the NTS are important in the regulation of the hypothalamus-pituitary-adrenal (HPA) axis in response to stress [811], and evidence indicates that brain GLP-1R signaling plays an integral role in the acute CNS response to stress and aversive stimuli [811]. Preproglucagon-positive neurons of the NTS have dense projections to other brain areas, including the hypothalamic PVN, where they innervate CRH-releasing neurons [812]. GLP-1R is expressed in neurons of the PVN [347] that co-localize with CRH [813]. Central administration of GLP-1 in rats and mice stimulates the HPA axis and increases secretion of corticosterone, and this is accompanied by increased cFOS immunoreactivity in CRH-positive neurons in the PVN $[668,698,814]$. In rodents and humans, peripherally administered 
GLP-1R agonists transiently stimulate the HPA axis and increase circulating levels of corticosterone, aldosterone, and ACTH [815-817]. Peripheral administration of exendin-4 or liraglutide increase circulating corticosterone in mice under basal conditions and also during a forced swimming test [818]. Mice with PVN-selective deletion of GLP$1 \mathrm{R}$ (using Sim1 cre) have impaired stress-induced HPA axis activation, and this is further accompanied by protection from stress-induced weight loss, reduced stress-induced cardiovascular endpoints and reduced anxiety-like behavior [813]. In rats, combined administration of dexamethasone and exendin-4 leads to a greater anorexigenic effect and greater weight loss relative to treatment with exendin-4 alone [819]. Similar results were reported in mice treated with GLP-1/ dexamethasone, an unimolecular peptide-nuclear hormone conjugate designed to optimize dexamethasone delivery into GLP-1R positive cells [820]. Ablation of hindbrain catecholamine neurons using antidopamine- $\beta$-hydroxylase-saporin (DSAP) blunts the ability of exendin4 to increase corticosterone secretion but interestingly potentiates the food intake inhibition by exendin-4 [819]. These data suggest that activation of the HPA axis antagonizes the satiation effect of GLP-1R agonism [819].

\section{GLP-1 EFFECTS ON LEARNING, MEMORY AND NEUROPROTECTION}

GLP-1R expression has also been demonstrated in the rat $[347,821]$ and mouse [822] hippocampus, a brain region implicated in spatial learning and memory [823,824]. In rats, central GLP-1R agonism improves several aspects of learning and memory, as demonstrated by improved performance in the Morris water maze test and by enhanced latency in the passive avoidance test [417]. GLP-1's improvement of learning and memory can be blocked by pretreatment with exendin $(9-39)$, and is absent in mice deficient for the GLP-1 receptor [417]. Targeted hippocampal restoration of GLP-1R expression in otherwise GLP-1R-deficient mice using AAV-mediated gene transfer reverses GLP-1 improvement of learning and memory in the passive avoidance test [417].

Central GLP-1R signaling has also been demonstrated to have neuroprotective effects. GLP-1 and exendin-4 enhance differentiation and neurite outgrowth in rat pheochromocytoma (PC12) cells and in human neuroblastoma SK-N-SH cells [825]. The neuroprotective effect of GLP-1R agonism is similar to that conferred by nerve growth factor (NGF) and is blocked when PC12 cells are co-incubated with exendin (9-39) [825]. Both GLP-1 and exendin-4 protect cultured hippocampal neurons against glutamate-induced apoptosis [826]. The time and severity of seizures induced by systemic administration of the neurotoxin kainic acid is enhanced in GLP-1R KO mice relative to WT controls whereas the destructive effect of kainic acid is ameliorated in the GLP1R KO mice upon targeted AAV-mediated restoration of hippocampal GLP-1R expression [417].

The molecular mechanisms underlying the neuroprotective effects of central GLP-1R agonism are, at least in part, mediated through the ability to increase cAMP formation and subsequently to enhance activation of PI3-kinase and ERK. GLP-1R agonism also increases CAMP levels in cultured hippocampal neurons [826] and in PC12 cells [825]. Pharmacological inhibition of either PI3-Kinase or ERK blocks the stimulatory effect GLP-1 and exendin-4 on neurite outgrowth in PC12 cells [825]. In PC12 cells, GLP-1 stimulation of neurite outgrowth is only partially silenced by treatment with the PKA inhibitor H89, indicating that CAMP-mediated activation of PI3K and ERK following GLP-1R agonism does not fully depend on PKA signaling, at least in these cells [825,827].
Huntington's disease (HD) is characterized by severe neurodegeneration that is caused by a mutation of the huntingtin protein (HTT) [828]. The neurotoxicity and neurodegeneration induced by the mutant HTT protein has been attributed to enhanced oxidative stress and accumulation of HTT due to inappropriate autophagic HTT clearance [829]. The prevalence of T2DM is elevated in patients with HD [830], suggesting that impaired insulin sensitivity might be a causal factor contributing to neurodegeneration in $\mathrm{HD}$ patients [830,831]. Overexpression of mutant HTT impairs insulin signaling and stimulates neuronal apoptosis in human SK-N-MC neuronal cells [831]. Treatment of SK-N-MC cells with liraglutide improves insulin sensitivity and enhances cell viability, potentially by mechanisms that include amelioration of neuronal glucotoxicity, improvement of oxidative stress and less aggregation of the mutant HTT through stimulation of AMPKmediated autophagy [831].

Alzheimer's disease (AD) is characterized by neurodegeneration of cholinergic neurons in the hippocampus. In a rat model of neurodegeneration, GLP-1 and exendin- 4 ameliorated neurodegeneration by reducing ibotenic acid-induced depletion of cholinergic cell bodies, as demonstrated by preservation of choline acetyltransferase immunoreactivity in basal forebrain cholinergic neurons [826]. In rats, administration of GLP-1R agonists into the hippocampus further prevented impairment of spatial learning and memory induced by central administration of amyloid $\beta(A \beta)$, a protein considered to play a causative role in impairment of learning and memory in Alzheimer disease [832,833]. Consistent with this, chronic GLP-1R agonism has been demonstrated in several mouse models of $A D$ to prevent the development of memory deficits [834-836]. Clinical studies evaluating the effect of GLP-1R agonism on neurodegeneration associated with $A D$ are scarce. $A D$ is frequently reported to be accompanied by decreased glucose transport across the BBB and 6 month treatment of $A D$ patients with liraglutide improved $B B B$ glucose transfer relative to placebo controls [837]. In a double blind placebo controlled study, 12 week treatment with liraglutide in patients at risk for $A D$ did not show cognitive differences between the study cohorts [838]. Other clinical trials evaluating the neuroprotective effects of GLP-1R agonism on $A D$ are currently ongoing [839]

GLP-1 analogs have also proven successful for the treatment of Parkinson's Disease (PD). PD is typically characterized by degeneration of dopaminergic neurons, a condition that can be mimicked in experimental animals by administration of the dopaminergic neurotoxin MPTP (1-Methyl-4-phenyl-1,2,3,6-tetrahydropyridin) [840-842]. In mice, 7-d infusion of exendin-4 into the lateral ventricle was sufficient to protect from MPTP-induced damage of the dopaminergic system and from the development of locomotor deficits that typically arise from dopamine deficiency [843]. Similar neuroprotective effects have been reported in various PD rodent models treated with GLP-1R agonists [844-849]. Consistent with this, exendin-4 increases survival and levels of tyrosine hydroxylase, the key enzyme for dopamine production, in primary neuronal cultures treated with the dopaminergic toxin 6-hydroxydopamine (6-OHDA) [843]. Several clinical studies have confirmed the potential of GLP-1R agonism to ameliorate clinical symptoms of PD, including long-lasting improvements of motor and cognitive function [850-853].

\section{GLP-1 EFFECTS ON BONE}

The first understanding of GLP-1 actions in skeletal physiology arose from GLP-1R KO mice, which exhibit a reduction in trabecular bone mass due to a higher osteoclast activity [854]. In rodents, but not in humans, activation of thyroid GLP-1R leads to C-cell stimulation in the 
thyroid [855,856], and involvement of calcitonin in GLP-1-mediated osteoclast activation has been suspected [854]. Furthermore, these animals exhibit profound modifications of bone matrix composition with a lower collagen maturity that jeopardizes the biomechanical response and hence contributes to skeletal fragility [857]. In comparison, GIPR KO animals present higher trabecular bone mass but significant lower biomechanical resistance due to alterations of tissue mineral density distribution and collagen maturity [858,859]. Double incretin receptor knock-out (DIRK0) animals revealed that the GLP-1/ GLP-1R pathway was important for the integrity of cortical but not trabecular bone [860].

GLP-1R agonists have been extensively used in animal models of skeletal fragility. In ovariectomy-induced bone loss, exenatide enhances bone formation due to osteoblast activation [857]. In hindlimbunloading rats, exenatide improves bone mass by promoting osteogenic differentiation of bone marrow stem cells [861]. In type-1 diabetic models, although liraglutide is less effective than GIP analogues in promoting osteoblast differentiation, it improves tissue material biomechanics by reducing collagen degradation [862]. In type-2 diabetic models (genetically-inherited or diet-induced obesity), exenatide significantly enhances bone strength by increasing trabecular bone mass, bone formation and trabecular microarchitecture. Exenatide significantly improves collagen maturity [863,864]. The use of the unimolecular triple agonist GIP-0xm is also significantly potent in improving bone strength and restoring trabecular bone mass [865]. However, its mechanism of action is linked to reduced bone formation and reduced osteoclast surfaces. GIP-0xm leads to changes in collagen lamellar orientation, improves collagen maturity and reduction in advanced glycation end-products in the bone matrix. However, it is not clear whether these ameliorations in bone strength result from activation of skeletal GLP-1 receptors or whether it requires extraskeletal receptors as observed for the GIP/GIPR pathway [866]. As such, a stable GIP agonist has been demonstrated to improve bone quality in rats [867], whereas DPP-4 inhibition positively modified bone composition but not bone microstructure in high fat fed mice [868]. Human studies report less compelling data on the effects of GLP-1 or its analogues in bone remodeling markers or fracture. In contrast to GLP-2 administration, subcutaneous injection of GLP-1 in healthy individuals had no effects on bone formation or resorption markers [869]. In non-diabetic, overweight men, GLP-1 infusion seems to reduce the extent of bone resorption in a similar way to GIP [787]. However, in obese, non-diabetic patients under anti-psychotic drugs, exenatide failed to modify significantly the pattern of bone remodeling [870]. In contrast, liraglutide was capable of enhancing bone formation in obese weight-reducing women while having no effects on bone resorption [871]. Data are more controversial in type-2 diabetes and although first reports did not find evidence of protective effects of the GLP-1 receptor agonist on bone fracture [872,873], more recent data suggest that liraglutide and lixisenatide, but not exenatide, contribute to reduce fractures in the diabetic population [874].

\section{PHARMACOLOGICAL USE OF GLP-1 ANALOGS TO TREAT OBESITY AND DIABETES}

The exogenous supplementation of native hormones, commonly purified from tissue homogenates, has led to numerous, seminal discoveries, most prominently the identification of insulin in 1921 [875] followed by the discovery of glucagon in 1923 [3]. Despite numerous such groundbreaking discoveries, including identifying the intestine as a source of incretins [56], this practice did not necessarily identify hormones capable of sufficiently lowering body weight.
Its anorexigenic and insulinotropic action make GLP-1 an appealing candidate for the treatment of obesity and diabetes. The pharmacological value of native GLP-1, however, is limited by a short half-life [182,185,876] and dose-limited adverse gastrointestinal effects $[734,877]$. A common approach to enhance the therapeutic benefits of GLP-1-based pharmacology is therefore to improve its pharmacokinetics. Biochemical modification of the native peptide has enhanced exposure and time of action to improve the metabolic efficacy at tolerable doses. Furthermore, these optimized molecules allow for less frequent administration, which places less burden on the patient and improved compliance.

\subsection{Optimized GLP-1 monoagonists}

Several structurally refined GLP-1 derivatives with improved bioavailability and sustained action have been developed for the treatment of T2DM [878]. These drugs include exenatide (Byetta ${ }^{\circledR}$, short-acting; and Bydureon $\AA$, long-acting, AstraZeneca), lixisenatide (Lyxumia ${ }^{\circ}$, Sanofi), liraglutide (Victoza $\AA$; Novo Nordisk), dulaglutide (Trulicity $\AA$; Eli Lilly \& Co), albiglutide (Tanzeum ${ }^{\circledR}$, GlaskoSmithKline), and semaglutide (Ozempic $\AA$, Novo Nordisk) [878].

Exenatide (Byetta ${ }^{\circledR}$ and Bydureon $\AA$, AstraZeneca), is a synthetic 39amino acid peptide (first discovered as exendin-4 in the saliva of the gila monster, Heloderma suspectum) (Figure 8) [879]. The first 30 amino residues of exendin-4 have $\sim 53 \%$ sequence homology to mammalian GLP-1, whereas the C-terminal nonapeptide extension has no similarities. Unlike GLP-1, exendin-4 has a glycine at the second amino acid position at the $\mathrm{N}$-terminus, which protects the peptide from DPP-4-mediated degradation and inactivation. In addition, exendin- 4 differs from mammalian GLP- 1 by a series of amino acids in the central and C-terminal domains that include Leu10, Lys12, Gln13, Met14, Glu16, Glu17, Tyr19, Arg20, Leu21, Glu24, Lys27, Asn28, and Gly30. The C-terminal end of exendin-4 is extended relative to GLP-1 by 9 amino acids. The C-terminal extension supports a secondary structure by formation of a tryptophan cage and improves potency at the GLP-1 receptor [880]. When administered either i.v., i.p., or S.c. in rats, the half-life of exendin-4 is 18-41 $\mathrm{min}, 125-174 \mathrm{~min}$, and 90-216 min, respectively [194]. Relative to GLP-1 (7-36 amide), the bioavailability of exendin-4 is nearly doubled from 36 to $67 \%$ for GLP-1 to $74-76 \%$ for exendin-4, with decelerated plasma clearance from 35 to $38 \mathrm{ml} / \mathrm{min}$ to $4-$ $8 \mathrm{ml} / \mathrm{min}$ [194]. These pharmacokinetic parameters support twicedaily administration in humans. In patients with T2DM, exenatide decreases fasting and post-prandial levels of blood glucose [881884], slows gastric emptying [885], reduces cardiovascular risk factors [886] and decreases body weight accompanied by suppression of food intake [885-887]. The placebo-subtracted weight loss from baseline induced by exenatide is typically in the range of low single-digit percentages. After 82 wk of treatment of T2DM patients with exenatide, the placebo-subtracted weight loss from baseline is approximately $5 \%$ [886]. A once-weekly formulation of exenatide has been developed by Amylin Pharmaceuticals and has been marketed by AstraZeneca since 2012 under the trade name Bydureon ${ }^{\circledR}$. The primary ingredient in the formulation that allows for extended-release is polyD,L-lactide-co-glycolic acid. Together with the other excipients of the formulation, the pharmacokinetics of exenatide are improved by a mechanism involving the formation of a depot of microspheres at the injection site, which slows dissipation from the injection site [888]. Compared to classical exenatide given twice daily (BID, the once-weekly (QW) formulation at a $2 \mathrm{mg}$ dose level provides a relatively stable plasma concentration at steady-state $(\sim 300 \mathrm{pg} / \mathrm{ml})$ because of the more continuous delivery of the drug, with equal (or 


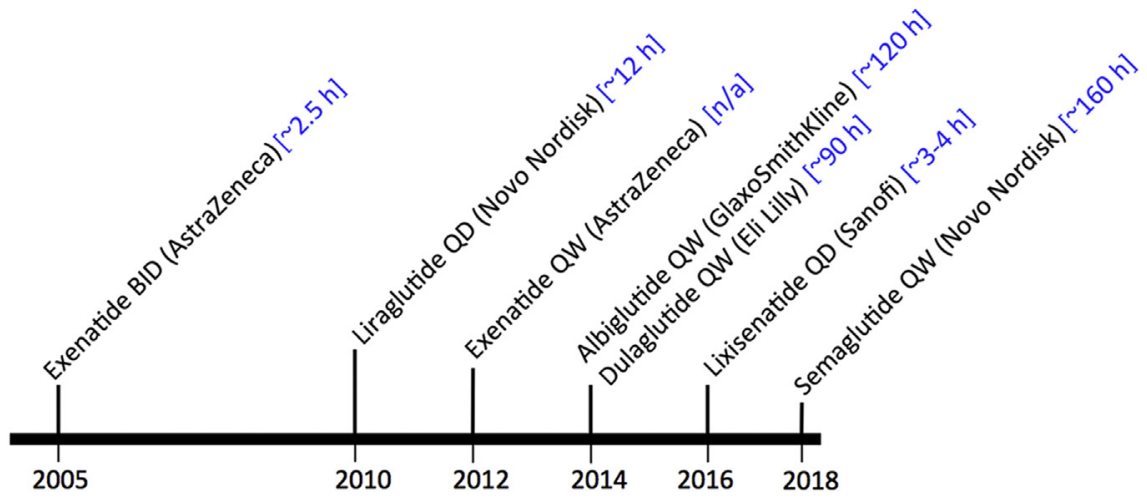

Figure 8: Timeline of GLP-1R agonists approved by the FDA for the treatment of diabetes. Numbers in parenthesis reflect the half-life of the molecules. For further explanations, please see text.

slightly improved) efficacy on glycemic control and body weight after 24 and 30 wk of treatment [888].

Lixisenatide (Adlyxin ${ }^{\circledR}$, Sanofi) (Figure 8 ) is an analogue of exenatide in which the proline at position 38 is omitted and six sequential lysine residues are added to the C-terminus [889]. Lixisenatide has comparable pharmacokinetic properties relative to exenatide and is, with a half-life of $\sim 3-4 \mathrm{~h}$, marketed for daily use in humans [890,891]. In patients with T2DM, the reduction in HbA1c is not overtly different between twice daily or once daily administration of lixisenatide [892]. Liraglutide at doses of 1.2 and $1.8 \mathrm{mg}$ (Victoza ${ }^{\circledR}$, Novo Nordisk) was approved for the treatment of type 2 diabetes in 2009 (Figure 8). Liraglutide is based on the native GLP-1 (7-37) sequence with a (conservative) substitution of lysine at position 28 with arginine. The alanine at the second position from the $\mathrm{N}$-terminus is preserved in liraglutide. However, the lysine at position 20 is linked via a gammaglutamic acid spacer to palmitic acid (C16:0), which via binding to albumin renders the drug less susceptible to DPP-4 proteolysis. The acylation also promotes multimeric formation, which decelerates diffusion from the site of injection [893]. In effect, the non-covalent binding of the fatty acid to albumin in effect prevents renal clearance. Altogether, these chemical modifications manifest in improved bioavailability and a half-life of $\sim 12 \mathrm{~h}$ [894], supporting once-daily injection in humans. Higher dose liraglutide $(3 \mathrm{mg})$ has been approved for the treatment of obesity since 2014 (Saxenda $($ )). The weight loss induced by liraglutide at $3 \mathrm{mg}$ is typically in the range of $\sim 5-10 \%$ after 52 wk of treatment of non-diabetic obese patients [895].

Semaglutide at doses of 0.5 and $1.0 \mathrm{mg}$ once weekly (Ozempic $\AA$, Novo Nordisk) has been approved for the treatment of T2DM and has been marketed since 2018 (Figure 8). Semaglutide is essentially an analogue of liraglutide. The DPP-4-sensitive alanine at the second Nterminal position in liraglutide is exchanged with an aminoisobuturic acid (Aib). Additionally, the palmitic (C16:0) fatty monoacid in liraglutide is exchanged to dicarboxylic-stearic acid (C18:0), and the link to this diacid-based fatty acid is extended relative to that in liraglutide with a synthetic spacer repeat [896]. These chemical modifications extend the half-life of semaglutide to $160 \mathrm{~h}$ upon subcutaneous injection in humans, supporting once-weekly administration [897]. Once-weekly injections of semaglutide dose-dependently induces body weight loss of up to $7 \%$ and reduces $\mathrm{HbA1c}$ by $1.8 \%$ after 40 wk of treatment in T2DM patients [898]. Semaglutide at a higher dose is being explored clinically for the treatment of non-diabetic obese patients, and an alternative oral formulation of semaglutide has completed Phase 3 clinical trials for type 2 diabetes. In patients with type 2 diabetes, semaglutide also reduces the risk for an adverse cardiovascular event, with decreased rates of MACE (Major Adverse Cardiovascular Events, comprising cardiovascular death, nonfatal myocardial infarction and stroke) [899].

Dulaglutide at 0.75 and $1.5 \mathrm{mg}$ (Trulicity ${ }^{\circledR}$, Eli Lilly \& $\mathrm{Co}$ ) has been approved for the treatment of T2DM patients. Dulaglutide consists of two GLP-1 molecules fused at their C-termini to a human Fc fragment of immunoglobulin G4 (IgG4) via a glycine and serine-based spacer. In each of the two GLP-1 motifs, certain exendin-4 residues are introduced to replace the native GLP-1 residues. A glycine at the second Nterminal position protects the molecule from DPP-4 inactivation while a glutamic acid at position 16 stabilizes the secondary structure and improves potency. A glycine substitution at position 30 , along with the native glycine at C-terminus of GLP-1 (7-37), serves as a lead-in sequence to the spacer that anchors the IgG4 Fc fragments. Together, these modifications improve bioavailability and decelerate renal clearance [900]. The resulting half-life of $90 \mathrm{~h}$ in humans renders the drug suitable for once weekly administration by injection (Figure 8) [901]. After 26 wk of once-weekly treatment of T2DM patients, dulaglutide at $1.5 \mathrm{mg}$ reduces body weight by nearly $3 \%$ from baseline and lowered $\mathrm{HbA} 1 \mathrm{C}$ by $1.4 \%$ [902].

Albiglutide is a head-to-tail tandem of two GLP-1 molecules in which the C-terminus of the first molecule is fused to the $\mathrm{N}$-terminus of the second. Each of the two GLP-1 molecules has a glycine substitution at the DPP-4 sensitive moieties. The C-terminus of the second GLP-1 is covalently fused to human albumin, thereby slowing renal clearance and augmenting the half-life to $\sim 120 \mathrm{~h}$ in humans [903], supporting once-weekly administration to humans. Albiglutide at $50 \mathrm{mg}$ (Tanzeum ${ }^{\circledR}$, GlaskoSmithKline) is approved for the treatment of type 2 diabetes but is no longer marketed.

A common feature of the GLP-1 analogs is the dose-dependent appearance of gastrointestinal adverse effects, most commonly nausea, vomiting, and diarrhea. Compared to short-acting GLP-1 analogs (such as exenatide bidaily), long-acting analogs are associated with less nausea and vomiting but with a greater prevalence of diarrhea [734]

\subsection{GLP-1-based combination therapies for individual dosing}

Chemical modification of native GLP-1 has been essential to advance the pharmacological value of GLP-1R agonism for the treatment of type 2 diabetes and obesity. Nonetheless, the use of these improved GLP$1 \mathrm{R}$ monoagonists to treat obesity is still limited, perhaps related to the 
level of body weight-lowering efficacy achieved with GLP-1R agonism alone. Higher doses of GLP-1R agonists can achieve clinically relevant weight loss, and greater increases in dose levels, in theory, can drive more weight-lowering efficacy. However, adverse gastrointestinal effects generally preclude the use of higher doses, even with adjusted uptitration dosing algorithms $[734,877]$.

Despite the appreciable weight lowering effect of semaglutide, single use of GLP-1R monoagonism may still have limited ability to drive body weight loss into the $\sim 20 \%$ range, GLP-1R agonism is a vital ingredient that can be used in adjunct with other body weight-lowering hormones to optimize weight loss at tolerable doses. It seems intuitive to assume that adjunct administration of several independent hormones, each given at a tolerable dose, would enhance metabolic outcome beyond what can be achieved by each hormone alone. In an ideal case, such co-administration would engage complementary signaling mechanisms such that the magnitude of metabolic benefits might be even greater than the sum of the individual monotherapies i.e., producing synergy. Historic examples of such a co-administration therapy for the treatment of obesity include the famous rainbow pills [878]. These pills, which enjoyed great popularity in the 1940's and 1950 's, comprised a series of weight lowering substances such as thyroid hormone, amphetamines, and laxatives. However, adverse effects on the cardiovascular and brain reward systems led to their discontinued use [878]. A more recent and controlled example of a polypharmacological anti-obesity pharmacotherapy is Qsymia ${ }^{\circledR}$ (Vivus Inc.), a combination of phentermine and topiramate. Qsymia, an extended-release formulation of this combination, was approved by the FDA for the treatment of obesity in 2012. Placebo-subtracted weight loss achieved by Qsymia is typically in the range of $5-10 \%$ after up to 56 wk of treatment $[904,905]$. The combination of GLP-1R analogs with basal insulin has yielded promising effects to accelerate metabolic and glycemic outcomes in patients with type-2 diabetes [906-908]. Relative to the monotherapies, the benefits of this combination therapy include a greater decrease of $\mathrm{HbA} 1 \mathrm{c}$ with an increased likelihood to achieve an $\mathrm{HbA} 1 \mathrm{c} \leq 7 \%$ [907]. Similar findings are reported for the combination of insulin with DPP-4 inhibitors [909]. Treatment of type-2 diabetic patients with a combination of the DPP-4 inhibitor sitagliptin and liraglutide showed no greater efficacy on glucose control relative to treatment with liraglutide alone, potentially because of GLP-1R saturation by the liraglutide monotherapy [910]. One study in type-2 diabetic patients reported improved glycemic control when exenatide was added to ongoing treatment with sitagliptin as compared to switching from sitagliptin to exenatide monotherapy [911].

Preclinical studies have evaluated the therapeutic potential of GLP-1 based polypharmacology for the treatment of obesity and diabetes. The various approaches include the combination of GLP-1R agonists with leptin [912,913], salmon calcitonin [914], PYY [915], CCK [916], insulin [917], adrenomedullin [918], and $\beta 3$-adrenergic receptor agonists [919], and antagonists at the cannabinoid receptor 1 (CB1) [920], or agonists at the receptors for melanocortin-4 (MC4R) [685] or farnesoid-X (FXR) [921]. In summary, a series of GLP-1-based combination therapies has been found to offer metabolic benefits greater than what is achieved by treatment with each compound alone.

\subsection{Unimolecular GLP-1-based polypharmacology}

Another more recently developed concept of polypharmacology is to combine some of the sequences of several structurally related hormones into a single entity of enhanced potency and sustained action. The concept underlying these unimolecular multi-agonists is that a single molecule with complementary signaling through more than one receptor, each of which offers beneficial effects on systems metabolism, would maximize metabolic outcomes at tolerable doses. This strategy has refined the toolbox of weight loss pharmacology, and several molecules with agonism at key metabolic receptors are, as reviewed below, currently in clinical evaluation. One might ask why a unimolecular multi-agonist is believed to be superior over the adjunct administration of the independent hormones? Apart from the possibility that acquiring regulatory approval is seemingly easier for a single molecule relative to a co-mixture of hormones, it must be considered that each molecule in a co-mixture has its own unique pharmacokinetic profile. Simultaneously injected hormones might differ in their half-life and time of action due to differences in rates of absorption, distribution, metabolism, and clearance, as well as the potential for drug-drug interaction. While these differences might compromise the optimal metabolic outcome of a co-mixture of separate molecule entities, a single molecule might bypass some of these limitations. With a single molecule approach, the ratio of activities of the constituents, however, will be fixed, whereas individual combination therapies present the potential benefit of being titratable to allow more optimal ratio between doses of the agonists. This is particularly important when one of the components has a narrow therapeutic window.

\subsubsection{GLP-1 dual- and triple-agonists}

The possibility of using glucagon as a constituent of a multi-functional single molecule for the treatment of chronic metabolic diseases was initially counterintuitive due to its established role in raising blood glucose as part of the counter-regulatory physiological response to defend against insulin-induced hypoglycemia. However, the catabolic qualities of glucagon, notably its effect to decrease hepatic fat and circulating lipids, could be leveraged for therapeutic benefit in metabolic diseases if its diabetogenic action were mitigated. The prospect emerged of using GLP-1 to buffer the hyperglycemic effect of glucagon while providing complementary mechanisms to lower body weight, most notably the effects of GLP-1 to suppress appetite to coincide with the energy expenditure effect of glucagon. Co-infusion of GLP-1 and glucagon to human subjects resulted in decreased food intake, likely secondary to GLP-1 action, and increased energy expenditure, suggested to be driven by glucagon action [783,785]. Chronic coadministration of selective mono-agonists to obese non-human primates resulted in significantly more body weight loss when compared to the individual treatments, thus demonstrating the translation of these acute effects observed in humans and the chronic effects observed in primates [673].

The structural similarity of GLP-1 and glucagon as well as of their respective receptors, along with the inherent low-potency promiscuity of these hormones at the other respective receptors (apart from the pancreas, where glucagon has recently been demonstrated to also act on GLP-1R [173]), suggested the possibility of integrating both activities into a sequence-intermixed single molecule hybrid. Preclinical proof of concept of enhanced body weight lowering relative to GLP-1 mono-therapy without impaired glycemic control was first demonstrated by two independent research efforts. Both of these GLP-1/ glucagon co-agonists, one based on a glucagon-derived backbone [922] and the other based on an oxyntomodulin-derived sequence [923], had superior weight loss in obese rodents relative to matched analogs that were selective in activity towards GLP-1R. Since these initial reports, other medicinal chemistry strategies have been employed to design unique GLP-1/glucagon co-agonists, including the use of exendin- 4 derived sequences [924], resulting in a rich pipeline of candidates that are advancing through clinical trials [925]. Two unique compounds have been evaluated in Phase 2 trials (SAR425899 and MEDI0382), and although dual-agonism has produced promising 
results on body weight and glycemic control in diabetic patients [926,927], it is not apparent that these co-agonists are superior to selective GLP-1R agonists.

The original rationale for combining GLP-1 and GIP, the other predominant incretin hormone, was to leverage the individual islet effects of each hormone to impart superior glycemic control. Co-infusion of GLP-1 and GIP to healthy human subjects has resulted in increased insulinotropic effects [928].

However, controversy still exists as to whether it is best to therapeutically promote or inhibit GIP action for beneficial effects on glycemic control and body weight [929-931] as both GIPR agonists [932] and antagonists [933,934] have decreased body weight in preclinical studies as individual treatments, but also to induce synergistic body weight loss when combined with GLP-1R agonists. It is important to note that recent independent confirmations on the prospects of GIPR agonism have been demonstrated with other GIP analogs [935,936]. As with glucagon, the structural similarity of GLP-1 and GIP provided the opportunity for rationally engineering single molecules of chimeric design [937]. The first reported GLP-1/GIP co-agonists, which had prolonged half-lives due to the use of fatty acylation or PEGylation, yielded synergistic effects, lowering body weight in obese rodents relative to the individual mono-agonist comparators [465] and having a greater insulinotropic effect in non-human primates. Tirzepatide (LY3298176), a once-weekly GLP-1/GIP co-agonist, was superior to a GLP-1R agonist (dulaglutide) in preclinical studies in terms of body weight loss and glycemic control improvement [938]. In the phase 2 study of type-2 diabetic patients, Tirzepatide produced a profound reduction in body weight and improved $\mathrm{HbA1c}$ levels, both of which were superior relative to effects of Dulaglutide in the active comparator arm [939], thus demonstrating the translation of the preclinical observations. Whether GIP-based co-agonists can provide greater maximal clinical efficacy, relative to optimized GLP-1R agonists remains unknown, and may be further evaluated in Phase 3 studies.

Based on the encouraging efficacy of GLP-1/glucagon and GLP-1/GIP co-agonists, it was rationalized that a single molecule with triple agonism at all three of these receptors might provide even greater efficacy than the respective co-agonists. The glucagon component would presumably confer energy expenditure benefits that are noticeably absent from GLP-1 and GIP pharmacology [465,932,938], and thus permit greater body weight lowering potency and efficacy. The GIP component is hypothesized to provide an additional mechanism to buffer against the hyperglycemic effects of glucagon [940], thus permitting triple agonists of higher potency at the glucagon receptor that in theory could drive more body weight loss. Indeed, a monomeric peptide with potent and balanced triple agonism of GLP1R, GIPR, and GcgR induced greater body weight loss than the respective co-agonists and mono-agonists in obese mice [466]. Similar preclinical benefits of a triple-acting GLP-1R, GIPR, and GCGR peptide have been observed in high-fat fed mice [941]. Readouts from the first clinical trials with triagonists will determine if such triple agonism has the curative potential that the preclinical data suggest.

20.3.2. GLP-1-based peptide-nuclear hormone conjugates

An alternative molecular format to achieve sequence-intermixed chimeric or hybridized peptides is single-molecule fusions or conjugates. As noted, the sequence similarity within the glucagon superfamily of peptides and the structural similarity within the secretin receptor family served as the molecular basis for engineering chimeric peptides with agonism at multiple receptors and with sizes comparable to the native peptides. Thus, engineering GLP-1-based multi-agonists that are hybridized sequences is likely restricted to similarly structured hormones. However, the theoretical combinations with GLP-1 can be extended to structurally diverse hormones because multi-valent chemical fusions or conjugates are possible where the second effector molecule can vary from small oligonucleotides and nuclear hormones to peptides and large proteins.

Single molecule fusions of GLP-1 to the peptide hormones gastrin, amylin, and cholecystokinin have been reported, as have fusions of GLP1 to larger proteins such as FGF21, and to inhibiting antibodies against PCSK9. GLP-1/gastrin fusions hold potential to re-granulate diseased islets and improve islet cell health in rodents genetically prone to develop diabetes [942]. Interestingly, modified GLP-1/gastrin fusion molecules that also incorporate bioactivity of the related gut hormone xenin have been found to have considerable preclinical efficacy [943,944]. GLP-1/ amylin fusions have potential in obese diabetic patients by capitalizing on two independent pharmacological functions to reduce food intake and lower blood glucose. Pre-clinical studies with GLP-1/amylin fusions have demonstrated body weight lowering efficacy in obese rodents [945]. Similarly, GLP-1/CCK fusions can capitalize on non-redundant food intake mechanisms to lower body weight. An optimized GLP-1/CCK fusion had enhanced efficacy to lower body weight in obese rodents relative to mono-agonist controls and their co-administration [946,947]. FGF21 analogues have been observed to potently lower triglycerides in T2DM patients [948] and to promote energy expenditure in rodents. Thus, GLP-1/FGF21 fusions have the potential, at least in rodents, to provide greater improvements in dyslipidemia than GLP-1R agonists and have the potential to drive greater body weight loss through the energy expenditure mechanism not evident with GLP-1 mono-therapy. GLP-1/ FGF21 fusions have been reported to dose-dependently lower body weight in obese mice, and co-administration of the respective monoagonists results in synergistic body weight loss in obese mice (abstracts). The combination of GLP-1 and anti-PCSK9 antibodies, like the GLP-1/FGF21 combinations, has great promise in diabetic patients at risk of cardiovascular disease due to effects on hypercholesterolemia. A pharmaceutically-optimized GLP-1/anti-PCSK9 antibody was reported to have potent weight lowering efficacy in obese rodents and to have potent effects to lower cholesterol in cynomolgous monkeys [949]. In a recent phase I clinical study, treatment of overweight and obese patients with GLP-1/anti-PCSK9 decreased LDL cholesterol but failed to improve glucose metabolism [950].

Oncolytic drug discovery research has been at the forefront of tissuetargeting strategies as a pharmacological tool to improve the efficacy of immunological agents by empowering them with classical chemotherapeutic mechanisms, a procedure which also results in an improved therapeutic window for the classical small molecule. By leveraging the high specificity of the antibodies and employing metastable covalent linkages of a cytotoxic payload, this effector molecule is targeted specifically to the antigen-producing cells. Once targeted, the cytotoxic agent is channeled into the cell via the antibody internalization process where it is released internally to provide a cytotoxic mechanism that is complementary to the immunological effects of the antibody. A similar concept has emerged in metabolic disease drug discovery research, although it is fundamentally different from the oncology field, because the effector molecule is not meant to drive a cell-killing effect but a cell-altering effect.

GLP-1R analogs have been leveraged for their relatively selective modalities of action in pancreatic islets and the central nervous system to precisely deliver cargo such as antisense oligonucleotides and nuclear hormones to these tissues. The aim is to alter the cellular physiology of these specific cell subtypes by the action of the effector molecule while simultaneously capitalizing on the therapeutic benefits of the GLP-1 targeting molecule. GLP-1/antisense conjugates have 
been developed to bypass a refractory nature of pancreatic $\beta$-cells to the uptake of antisense oligonucleotides while harnessing the untapped potential of these oligonucleotides to repress the expression of pathological intracellular targets [951]. Conjugates of particular antisense oligonucleotides to a GLP-1 analog enhanced the productive uptake of the oligonucleotide in GLP-1R expressing cells to elicit selective and effective gene silencing in vitro and in vivo with limited offtarget accumulation of the oligonucleotide. Although the therapeutic benefits have yet to be reported for a specific GLP-1/antisense molecule designed to deliver a therapeutically-relevant antisense, the data suggest that GLP-1R is a suitable vector for in vivo tissue targeting, cellular internalization, and intracellular deposition of a cargo moiety. However, a comprehensive application of GLP-1R mediated targeting has yet to be demonstrated though translational pharmacology experiments.

In other pre-clinical experiments, GLP-1 analogs have been utilized to selectively deliver nuclear hormone payloads to cells in a targeted manner. Estrogen has pleotropic, beneficial effects on metabolism yet the reproductive endocrine toxicity and tumorigenic potential limits its use in chronic settings other than hormone replacement therapy. Emerging preclinical data suggest that a GLP-1/estrogen conjugate enhances the anorectic, body weight lowering, insulinotropic, and islet preservation effects in mice relative to the GLP-1 analog or estrogen alone $[467,952,953]$. Importantly, this GLP-1/estrogen conjugate was devoid of the uterotrophic effect evident with estrogen alone, demonstrating an improved therapeutic index mediated by the GLP-1 component. GLP-1R targeting has also been leveraged to deliver the anti-inflammatory properties of dexamethasone to metabolically relevant tissues. Although glucocorticoids are potent anti-inflammatory molecules, chronic treatment oftentimes results in compromised metabolic control, notably due to hepatic action to increase glucose production. In mice, a GLP-1/dexamethasone conjugate circumvented action in the liver and skeletal system to avoid the deleterious effects on glycemic control and bone turnover, respectively, that is evident with systemic dexamethasone action [820]. This GLP-1/ dexamethasone conjugate improved the body weight lowering potency of the GLP-1 analog alone, partially by alleviating surrogate markers of hypothalamic inflammation. Despite these provocative preclinical findings suggesting a targeting phenomenon that results in enhanced efficacy and improved therapeutic index for these nuclear hormone conjugates, more studies are required to delineate the mechanism for the intracellular delivery and release of the cargo along with precise pharmacokinetic analyses. Furthermore, this targeting principle requires additional validation, particularly in the context of human biology, before tangible conclusions regarding its applicability to clinical pharmacology can be made.

\section{OUTLOOK}

Depending on the molecule (native or recombinant long-acting) and the route of administration, GLP-1 analogs have broad pleiotropic action on metabolism. Among the numerous beneficial effects mediated by GLP$1 \mathrm{R}$ agonists are regulation of blood glucose, lowering of body weight via inhibition of food intake and the decrement of gastric motility, stimulation of cell proliferation, reduction of inflammation and apoptosis, improvement of cardiovascular function, and neuroprotection. Recombinant GLP-1R analogs with improved pharmacokinetics and sustained action are successful in the treatment of type-2 diabetes, and there is considerable and ongoing effort to further optimize their action profile to improve therapeutic outcome and patient compliance. Novel peptides that combine the pharmacology of GLP-1 with that of other gut peptides are in clinical evaluation for the treatment of diabetes and obesity. While long-term clinical studies are ongoing, we can be carefully optimistic that improved GLP-1-based pharmacology can one day be safely applied to further lower body weight relative to currently available GLP-1R agonists.

\section{ACKNOWLEDGEMENTS}

This work was supported by grants from the Alexander von Humboldt Foundation, Helmholtz Alliance ICEMED, and Helmholtz Initiative on Personalized Medicine iMed by Helmholtz Association, and Helmholtz cross-program topic "Metabolic Dysfunction". This work was further supported by German Research Foundation Grants DFGTS226/1-1, DFG-TS226/3-1, and DFG-TR152 (project no. 239283807), land the European Research Council ERC AdG HypoFlam 695054, and German Center for Diabetes Research (DZD e.V.). DJD is supported by a Banting and Best Diabetes Centre-Novo Nordisk Chair in Incretin Biology and by CIHR Foundation grant 154321. The figures were made using material provided by Servier Medical Art (Servier), under consideration of a Creative Commons Attribution 3.0 Unported License.

\section{CONFLICT OF INTEREST}

KS, SCW, TWS, WL, PF, FR, FG, JFH, SRB, DD'A, AF, JJH, PDT and AP have nothing to disclose. DAS receives research support from Novo Nordisk and Medimmune. DJD served as an advisor or consultant or speaker within the past 12 months to Forkhead Biotherapeutics, Intarcia Therapeutics, Kallyope, Merck Research Laboratories, Novo Nordisk, Pfizer, and Sanofi. Neither DJD or his family members hold stock directly or indirectly in any of these companies. MTC is employee of Novo Nordisk, where part of the business is related GLP-1R agonists. MAN has been member on advisory boards or has consulted with AstraZeneca, Boehringer Ingelheim, Eli Lilly, Fractyl, GlaxoSmithKline, Menarini/Berlin Chemie, Merck, Sharp \& Dohme, and Novo Nordisk. He has received grant support from AstraZeneca, Eli Lilly, Menarini/Berlin-Chemie, Merck, Sharp \& Dohme, Novartis Pharma and Novo Nordisk. He has also served on the speakers' bureau of AstraZeneca, Boehringer Ingelheim, Eli Lilly, Menarini/Berlin Chemie, Merck, Sharp \& Dohme, Novo Nordisk, and Sun Pharma. HJG is consultant and member of the Global Obesity Advisory Board of Novo Nordisk. RS is consultant or receives research support from Novo Nordisk, Sanofi, Johnson \& Johnson, Zafgen, Kallyope, Scohia, AstraZeneca, Ironwood Pharma, Redesign Health, Pfizer and GuidePoint Consultants. RDDiM and BF have IP at Indiana University and employment with Novo Nordisk that pertains to the subject. MHT is a scientific advisor for Erx Biotech. TDM receives grant support from Novo Nordisk and Sanofi. JJM has received lecture honoraria and consulting fees from the AstraZeneca, Berlin-Chemie, Boehringer Ingelheim, Eli Lilly, Merck Sharp \& Dohme, Novo Nordisk, Novartis, and Sanofi; has received reimbursement of congress participation fees and travel expenses from Merck Sharp \& Dohme, Novo Nordisk, and Sanofi; and has initiated projects supported by Boehringer-Ingelheim, Merck Sharp \& Dohme, Novo Nordisk, and Sanofi.

\section{REFERENCES}

[1] Collip, J.B., 1923. Delayed manifestation of the physiological effects of insulin following the administration of certain pancreatic extracts. American Journal of Physiology 63:391.

[2] Fisher, N.F., 1923. Preparation of insulin. American Journal of Physiology 67: 57.

[3] Kimball, C., Murlin, J., 1923. Aqueous extracts of pancreas III. Some precipitation reactions of insulin. Journal of Biological Chemistry 58:337-348.

[4] Muller, T.D., Finan, B., Clemmensen, C., DiMarchi, R.D., Tschop, M.H., 2017. The new biology and pharmacology of glucagon. Physiological Reviews 97: $721-766$. 
[5] Unger, R.H., Orci, L., 1976. Physiology and pathophysiology of glucagon. Physiological Reviews 56:778-826.

[6] Bürger, M., Brandt, W., 1935. Über das Glukagon (die hyperglykämisierende Substanz der Pankreas). Zeitschrift für die gesamte experimentelle Medizin 96:375.

[7] Bürger, M., Kramer, H., 1929. Über den hepatischen Angriffspunkt des Insulins. Die primäre Paradoxe Insulinhyperglykämie. Zeitschrift für die gesamte experimentelle Medizin 65:487.

[8] Bürger, M., Kramer, H., 1929. Primäre Hyperglykämie und Glykogenveränderung der Leber als Folge intraportaler Insulininjektion nach Untersuchungen am Hund. Zeitschrift für die gesamte experimentelle Medizin 67:441.

[9] Exton, J.H., Park, C.R., 1968. Control of gluconeogenesis in liver. II. Effects of glucagon, catecholamines, and adenosine 3',5'-monophosphate on gluconeogenesis in the perfused rat liver. Journal of Biological Chemistry 243: 4189-4196.

[10] Garcia, A., Williamson, J.R., Cahill Jr., G.F., 1966. Studies on the perfused rat liver. II. Effect of glucagon on gluconeogenesis. Diabetes 15:188-193.

[11] Struck, E., Ashmore, J., Wieland, 0., 1965. Stimulation of gluconeogenesis by long chain fatty acids and glucagon. Biochemische Zeitschrift 343:107110.

[12] Ui, M., Claus, T.H., Exton, J.H., Park, C.R., 1973. Studies on the mechanism of action of glucagon on gluconeogenesis. Journal of Biological Chemistry 248:5344-5349.

[13] Samols, E., Marri, G., Marks, V., 1965. Promotion of insulin secretion by glucagon. Lancet 2:415-416.

[14] Vuylsteke, C.A., Cornelius, G., deDuve, C., 1952. Influence du traitment au cobalt sur le contenu en facteur HG du pancreas de cobaye. Archives Internationales de Physiologie 60:128.

[15] Fodden, J.H., Read, W.0., 1954. The activity of extracted pancreatic hyperglycemic-glycogenolytic factor after cobaltous chloride and synthalin A. Endocrinology 54:303.

[16] Hellerstrom, C., Hellman, B., Petersson, B., Alm, G., 1964. The two types of pancreatic alpha cells and their relation to glucagon secretion. In: Brolin, S.E., Hellman, B., Knutson, H. (Eds.), Structure and Metabolism of the Pancreatic Islets. Edinburgh \& New York: Pergamon Press Ltd. p. 117-29.

[17] Hellerstrom, C., Hellman, B., 1962. Reactions of the two types of A cells in the islets of Langerhans after administration of glucagon. Acta Endocrinologica (Copenhagen) 41:116-122.

[18] Petersson, B., Hellman, B., 1963. Effects of long term administration of glucagon on the pancreatic islet tissue of rats and Guinea-pigs. Acta Endocrinologica (Copenhagen) 44:139-149.

[19] Hellman, B., Lernmark, A., 1969. Inhibition of the in vitro secretion of insulin by an extract of pancreatic alpha-1 cells. Endocrinology 84:1484-1488.

[20] Unger, R.H., Eisentraut, A.M., Mc, C.M., Keller, S., Lanz, H.C., Madison, L.L., 1959. Glucagon antibodies and their use for immunoassay for glucagon. Proceedings of The Society for Experimental Biology and Medicine 102:621623.

[21] Samols, E., Marks, V., 1967. New conceptions on the functional significance of glucagon (pancreatic and extra-pancreatic). Journees Annuelles de Diabetologie de l'Hotel-Dieu 7:43-66.

[22] Moody, A.J., Markussen, J., Fries, A.S., Steenstrup, C., Sundby, F., 1970. The insulin releasing activites of extracts of pork intestine. Diabetologia 6: $135-140$.

[23] Murphy, R.F., Elmore, D.T., Buchanan, K.D., 1971. Isolation of glucagon-like immunoreactivity of gut by affinity chromatography. Biochemical Journal 125 : 61P-62P.

[24] Unger, R.H., Eisentraut, A.M., Sims, K., McCall, M.S., Madison, L.L., 1961. Sites of origin of glucagon in dogs and humans. Clinical Research 9:53.

[25] Valverde, I., Rigopoulou, D., Marco, J., Faloona, G.R., Unger, R.H., 1970. Characterization of glucagon-like immunoreactivity (GLI). Diabetes 19:614623.
[26] Unger, R.H., Ketterer, H., Eisentraut, A.M., 1966. Distribution of immunoassayable glucagon in gastrointestinal tissues. Metabolism 15:865-867.

[27] Unger, R.H., Ohneda, A., Valverde, I., Eisentraut, A.M., Exton, J., 1968. Characterization of the responses of circulating glucagon-like immunoreactivity to intraduodenal and intravenous administration of glucose. Journal of Clinical Investigation 47:48-65.

[28] Valverde, I., Rigopoulou, D., Exton, J., Ohneda, A., Eisentraut, A., Unger, R.H., 1968. Demonstration and characterization of a second fraction of glucagonlike immunoreactivity in jejunal extracts. The American Journal of the Medical Sciences 255:415-420.

[29] Grimelius, L., Capella, C., Buffa, R., Polak, J.M., Pearse, A.G., Solcia, E., 1976. Cytochemical and ultrastructural differentiation of enteroglucagon and pancreatic-type glucagon cells of the gastrointestinal tract. Virchows Archiv B Cell Pathology 20:217-228.

[30] Buffa, R., Capella, C., Fontana, P., Usellini, L., Solcia, E., 1978. Types of endocrine cells in the human colon and rectum. Cell and Tissue Research 192:227-240.

[31] Rigopoulou, D., Valverde, I., Marco, J., Faloona, G., Unger, R.H., 1970. Large glucagon immunoreactivity in extracts of pancreas. Journal of Biological Chemistry 245:496-501.

[32] Tung, A.K., Zerega, F., 1971. Biosynthesis of glucagon in isolated pigeon islets. Biochemical and Biophysical Research Communications 45:387-395.

[33] Hellerstrom, C., Howell, S.L., Edwards, J.C., Andersson, A., 1972. An investigation of glucagon biosynthesis in isolated pancreatic islets of Guinea pigs. FEBS Letters 27:97-101.

[34] Patzelt, C., Tager, H.S., Carroll, R.J., Steiner, D.F., 1979. Identification and processing of proglucagon in pancreatic islets. Nature 282:260-266.

[35] Patzelt, C., Schug, G., 1981. The major proglucagon fragment: an abundant islet protein and secretory product. FEBS Letters 129:127-130.

[36] Holst, J.J., 1982. Evidence that enteroglucagon (II) is identical with the Cterminal sequence (residues 33-69) of glicentin. Biochemical Journal 207: $381-388$.

[37] Jacobsen, H., Demandt, A., Moody, A.J., Sundby, F., 1977. Sequence analysis of porcine gut GLI-1. Biochimica et Biophysica Acta 493:452-459.

[38] Sundby, F., Jacobsen, H., Moody, A.J., 1976. Purification and characterization of a protein from porcine gut with glucagon-like immunoreactivity. Hormone and Metabolic Research 8:366-371.

[39] Thim, L., Moody, A.J., 1981. The amino acid sequence of porcine glicentin. Peptides 2(Suppl. 2):37-39.

[40] Holst, J.J., 1980. Evidence that glicentin contains the entire sequence of glucagon. Biochemical Journal 187:337-343.

[41] Moody, A.J., Holst, J.J., Thim, L., Jensen, S.L., 1981. Relationship of glicentin to proglucagon and glucagon in the porcine pancreas. Nature 289: 514-516.

[42] Bataille, D., Tatemoto, K., Gespach, C., Jornvall, H., Rosselin, G., Mutt, V., 1982. Isolation of glucagon-37 (bioactive enteroglucagon/oxyntomodulin) from porcine jejuno-ileum. Characterization of the peptide. FEBS Letters 146: 79-86.

[43] Thim, L., Moody, A.J., 1982. Purification and chemical characterization of a glicentin-related pancreatic peptide (proglucagon fragment) from porcine pancreas. Biochimica et Biophysica Acta 703:134-141.

[44] Lund, P.K., Goodman, R.H., Dee, P.C., Habener, J.F., 1982. Pancreatic preproglucagon CDNA contains two glucagon-related coding sequences arranged in tandem. Proceedings of the National Academy of Sciences of the United States of America 79:345-349.

[45] Lund, P.K., Goodman, R.H., Habener, J.F., 1981. Pancreatic preproglucagons are encoded by two separate mRNAs. Journal of Biological Chemistry 256:6515-6518.

[46] Lund, P.K., Goodman, R.H., Montminy, M.R., Dee, P.C., Habener, J.F., 1983. Anglerfish islet pre-proglucagon II. Nucleotide and corresponding amino acid sequence of the cDNA. Journal of Biological Chemistry 258:3280-3284. 
[47] Heinrich, G., Gros, P., Habener, J.F., 1984. Glucagon gene sequence. Four of six exons encode separate functional domains of rat pre-proglucagon. Journal of Biological Chemistry 259:14082-14087.

[48] Heinrich, G., Gros, P., Lund, P.K., Bentley, R.C., Habener, J.F., 1984. Preproglucagon messenger ribonucleic acid: nucleotide and encoded amino acid sequences of the rat pancreatic complementary deoxyribonucleic acid. Endocrinology 115:2176-2181.

[49] Bell, G.I., Santerre, R.F., Mullenbach, G.T., 1983. Hamster preproglucagon contains the sequence of glucagon and two related peptides. Nature 302: $716-718$.

[50] Lopez, L.C., Frazier, M.L., Su, C.J., Kumar, A., Saunders, G.F., 1983. Mammalian pancreatic preproglucagon contains three glucagon-related peptides. Proceedings of the National Academy of Sciences of the United States of America 80:5485-5489.

[51] Bell, G.I., Sanchez-Pescador, R., Laybourn, P.J., Najarian, R.C., 1983. Exon duplication and divergence in the human preproglucagon gene. Nature 304: $368-371$.

[52] Mojsov, S., Heinrich, G., Wilson, I.B., Ravazzola, M., Orci, L., Habener, J.F., 1986. Preproglucagon gene expression in pancreas and intestine diversifies at the level of post-translational processing. Journal of Biological Chemistry 261:11880-11889.

[53] Orskov, C., Holst, J.J., Knuhtsen, S., Baldissera, F.G., Poulsen, S.S., Nielsen, 0.V., 1986. Glucagon-like peptides GLP-1 and GLP-2, predicted products of the glucagon gene, are secreted separately from pig small intestine but not pancreas. Endocrinology 119:1467-1475.

[54] Orskov, C., Holst, J.J., Poulsen, S.S., Kirkegaard, P., 1987. Pancreatic and intestinal processing of proglucagon in man. Diabetologia 30:874-881.

[55] Moore, B., 1906. On the treatment of Diabetus mellitus by acid extract of Duodenal Mucous Membrane. Biochemical Journal 1:28-38.

[56] Zunz, E., La Barre, J., 1929. Contributiona a l'etude des variations physiologiques de la secretion interne du pancreas: relations entre les secretions externe et interne du pancreas. Archives Internationales de Physiologie et de Biochimie 31:20-44.

[57] La Barre, J., 1932. Sur les possibilite's d'un traitement du diabète par l'incrétine. Bulletin de l'Academie Royale de Medecine de Belgique 12:620634.

[58] Yalow, R.S., Berson, S.A., 1959. Assay of plasma insulin in human subjects by immunological methods. Nature 184(Suppl. 21):1648-1649.

[59] Yalow, R.S., Berson, S.A., 1960. Immunoassay of endogenous plasma insulin in man. Journal of Clinical Investigation 39:1157-1175.

[60] Dupre, J., Beck, J.C., 1966. Stimulation of release of insulin by an extract of intestinal mucosa. Diabetes 15:555-559.

[61] Elrick, H., Stimmler, L., Hlad Jr., C.J., Arai, Y., 1964. Plasma insulin response to oral and intravenous glucose administration. The Journal of Cinical Endocrinology and Metabolism 24:1076-1082.

[62] McIntyre, N., Holdsworth, C.D., Turner, D.S., 1964. New interpretation of oral glucose tolerance. Lancet 2:20-21.

[63] Perley, M.J., Kipnis, D.M., 1967. Plasma insulin responses to oral and intravenous glucose: studies in normal and diabetic sujbjects. Journal of Clinical Investigation 46:1954-1962.

[64] Nauck, M.A., Meier, J.J., 2018. Incretin hormones: their role in health and disease. Diabetes Obesity and Metabolism 20(Suppl. 1):5-21.

[65] Nauck, M.A., Meier, J.J., 2016. The incretin effect in healthy individuals and those with type 2 diabetes: physiology, pathophysiology, and response to therapeutic interventions. Lancet Diabetes Endocrinology 4:525-536.

[66] Samols, E., Tyler, J., Marri, G., Marks, V., 1965. Stimulation of glucagon secretion by oral glucose. Lancet 2:1257-1259.

[67] Gregory, R.A., 1977. An assessment of Viktor Mutt's contributions. Gastroenterology $72: 786-787$.
[68] Brown, J.C., Dryburgh, J.R., 1971. A gastric inhibitory polypeptide. II. The complete amino acid sequence. Canadian Journal of Biochemistry 49:867872.

[69] Brown, J.C., Mutt, V., Pederson, R.A., 1970. Further purification of a polypeptide demonstrating enterogastrone activity. Journal of Physiology 209: $57-64$.

[70] Brown, J.C., Pederson, R.A., Jorpes, E., Mutt, V., 1969. Preparation of highly active enterogastrone. Canadian Journal of Physiology and Pharmacology 47: $113-114$.

[71] Dupre, J., Ross, S.A., Watson, D., Brown, J.C., 1973. Stimulation of insulin secretion by gastric inhibitory polypeptide in man. The Journal of Cinical Endocrinology and Metabolism 37:826-828.

[72] Schafer, R., Schatz, H., 1979. Stimulation of (Pro-)insulin biosynthesis and release by gastric inhibitory polypeptide in isolated islets of rat pancreas. Acta Endocrinologica (Copenhagena) 91:493-500.

[73] Schauder, P., Brown, J.C., Frerichs, H., Creutzfeldt, W., 1975. Gastric inhibitory polypeptide: effect on glucose-induced insulin release from isolated rat pancreatic islets in vitro. Diabetologia 11:483-484.

[74] Szecowka, J., Grill, V., Sandberg, E., Efendic, S., 1982. Effect of GIP on the secretion of insulin and somatostatin and the accumulation of cyclic AMP in vitro in the rat. Acta Endocrinologica (Copenhagen) 99:416-421.

[75] Taminato, T., Seino, Y., Goto, Y., Inoue, Y., Kadowaki, S., 1977. Synthetic gastric inhibitory polypeptide. Stimulatory effect on insulin and glucagon secretion in the rat. Diabetes 26:480-484.

[76] Adrian, T.E., Bloom, S.R., Hermansen, K., Iversen, J., 1978. Pancreatic polypeptide, glucagon and insulin secretion from the isolated perfused canine pancreas. Diabetologia 14:413-417.

[77] Brunicardi, F.C., Druck, P., Seymour, N.E., Sun, Y.S., Elahi, D., Andersen, D.K., 1990. Selective neurohormonal interactions in islet cell secretion in the isolated perfused human pancreas. Journal of Surgical Research 48:273-278.

[78] Ebert, R., Unger, H., Creutzfeldt, W., 1983. Preservation of incretin activity after removal of gastric inhibitory polypeptide (GIP) from rat gut extracts by immunoadsorption. Diabetologia 24:449-454.

[79] Lauritsen, K.B., Moody, A.J., Christensen, K.C., Lindkaer Jensen, S., 1980. Gastric inhibitory polypeptide (GIP) and insulin release after small-bowe resection in man. Scandinavian Journal of Gastroenterology 15:833-840.

[80] Holst, J.J., Orskov, C., Nielsen, O.V., Schwartz, T.W., 1987. Truncated glucagon-like peptide I, an insulin-releasing hormone from the distal gut. FEBS Letters 211:169-174.

[81] Mojsov, S., Weir, G.C., Habener, J.F., 1987. Insulinotropin: glucagon-like peptide I (7-37) co-encoded in the glucagon gene is a potent stimulator of insulin release in the perfused rat pancreas. Journal of Clinical Investigation 79:616-619.

[82] Drucker, D.J., Philippe, J., Mojsov, S., Chick, W.L., Habener, J.F., 1987. Glucagon-like peptide I stimulates insulin gene expression and increases cyclic AMP levels in a rat islet cell line. Proceedings of the National Academy of Sciences of the United States of America 84:3434-3438.

[83] Kreymann, B., Williams, G., Ghatei, M.A., Bloom, S.R., 1987. Glucagon-like peptide-1 7-36: a physiological incretin in man. Lancet 2:1300-1304.

[84] Drucker, D.J., Asa, S., 1988. Glucagon gene expression in vertebrate brain. Journal of Biological Chemistry 263:13475-13478.

[85] Han, V.K., Hynes, M.A., Jin, C., Towle, A.C., Lauder, J.M., Lund, P.K., 1986. Cellular localization of proglucagon/glucagon-like peptide I messenger RNAs in rat brain. Journal of Neuroscience Research 16:97-107.

[86] Jin, S.L., Han, V.K., Simmons, J.G., Towle, A.C., Lauder, J.M., Lund, P.K., 1988. Distribution of glucagonlike peptide I (GLP-I), glucagon, and glicentin in the rat brain: an immunocytochemical study. Journal of Comparative Neurology 271:519-532. 
[87] Sandoval, D.A., D’Alessio, D.A., 2015. Physiology of proglucagon peptides: role of glucagon and GLP-1 in health and disease. Physiological Reviews 95: 513-548.

[88] Nian, M., Drucker, D.J., Irwin, D., 1999. Divergent regulation of human and rat proglucagon gene promoters in vivo. American Journal of Physiology 277: G829-G837.

[89] Philippe, J., 1991. Structure and pancreatic expression of the insulin and glucagon genes. Endocrine Reviews 12:252-271.

[90] Holt, M.K., Richards, J.E., Cook, D.R., Brierley, D.l., Williams, D.L., Reimann, F., et al., 2019. Preproglucagon neurons in the nucleus of the solitary tract are the main source of brain GLP-1, mediate stress-induced hypophagia, and limit unusually large intakes of food. Diabetes 68:21-33.

[91] Drucker, D.J., 1990. Glucagon and the glucagon-like peptides. Pancreas 5: 484-488.

[92] Tang-Christensen, M., Larsen, P.J., Goke, R., Fink-Jensen, A., Jessop, D.S., Moller, M., et al., 1996. Central administration of GLP-1-(7-36) amide inhibits food and water intake in rats. American Journal of Physiology 271:R848R856.

[93] Lee, Y.C., Brubaker, P.L., Drucker, D.J., 1990. Developmental and tissuespecific regulation of proglucagon gene expression. Endocrinology 127: 2217-2222.

[94] Jin, T., 2008. Mechanisms underlying proglucagon gene expression. Journal of Endocrinology 198:17-28.

[95] Efrat, S., Teitelman, G., Anwar, M., Ruggiero, D., Hanahan, D., 1988. Glucagon gene regulatory region directs oncoprotein expression to neurons and pancreatic alpha cells. Neuron 1:605-613.

[96] Lee, Y.C., Asa, S.L., Drucker, D.J., 1992. Glucagon gene 5'-flanking sequences direct expression of simian virus 40 large $T$ antigen to the intestine, producing carcinoma of the large bowel in transgenic mice. Journal of Biological Chemistry 267:10705-10708.

[97] Zhou, L., Nian, M., Gu, J., Irwin, D.M., 2006. Intron 1 sequences are required for pancreatic expression of the human proglucagon gene. American Journal of Physiology Regulatory Integrative and Comparative Physiology 290:R634R641.

[98] Jin, T., Drucker, D.J., 1996. Activation of proglucagon gene transcription through a novel promoter element by the caudal-related homeodomain protein cdx-2/3. Molecular and Cellular Biology 16:19-28.

[99] Knepel, W., Jepeal, L., Habener, J.F., 1990. A pancreatic islet cellspecific enhancer-like element in the glucagon gene contains two domains binding distinct cellular proteins. Journal of Biological Chemistry 265:8725-8735.

[100] Gosmain, Y., Cheyssac, C., Heddad Masson, M., Dibner, C., Philippe, J., 2011. Glucagon gene expression in the endocrine pancreas: the role of the transcription factor Pax6 in alpha-cell differentiation, glucagon biosynthesis and secretion. Diabetes Obesity and Metabolism 13(Suppl. 1):31-38.

[101] Gevrey, J.C., Malapel, M., Philippe, J., Mithieux, G., Chayvialle, J.A., Abello, J., et al., 2004. Protein hydrolysates stimulate proglucagon gene transcription in intestinal endocrine cells via two elements related to cyclic AMP response element. Diabetologia 47:926-936.

[102] Herzig, S., Fuzesi, L., Knepel, W., 2000. Heterodimeric Pbx-Prep1 homeodomain protein binding to the glucagon gene restricting transcription in a cell type-dependent manner. Journal of Biological Chemistry 275:27989-27999.

[103] Philippe, J., Drucker, D.J., Habener, J.F., 1987. Glucagon gene transcription in an islet cell line is regulated via a protein kinase C-activated pathway. Journal of Biological Chemistry 262:1823-1828.

[104] Gauthier, B.R., Schwitzgebel, V.M., Zaiko, M., Mamin, A., Ritz-Laser, B., Philippe, J., 2002. Hepatic nuclear factor-3 (HNF-3 or Foxa2) regulates glucagon gene transcription by binding to the G1 and G2 promoter elements. Molecular Endocrinology 16:170-183.

[105] Gosmain, Y., Avril, I., Mamin, A., Philippe, J., 2007. Pax-6 and C-Maf functionally interact with the alpha-cell-specific DNA element G1 in vivo to promote glucagon gene expression. Journal of Biological Chemistry 282: 35024-35034.

[106] Andersen, F.G., Heller, R.S., Petersen, H.V., Jensen, J., Madsen, O.D., Serup, P., 1999. Pax6 and Cdx2/3 form a functional complex on the rat glucagon gene promoter G1-element. FEBS Letters 445:306-310.

[107] St-Onge, L., Sosa-Pineda, B., Chowdhury, K., Mansouri, A., Gruss, P., 1997. Pax6 is required for differentiation of glucagon-producing alpha-cells in mouse pancreas. Nature 387:406-409.

[108] Trinh, D.K., Zhang, K., Hossain, M., Brubaker, P.L., Drucker, D.J., 2003. Pax6 activates endogenous proglucagon gene expression in the rodent gastrointestinal epithelium. Diabetes 52:425-433.

[109] Hill, M.E., Asa, S.L., Drucker, D.J., 1999. Essential requirement for Pax6 in control of enteroendocrine proglucagon gene transcription. Molecular Endocrinology 13:1474-1486.

[110] Drucker, D.J., Philippe, J., Jepeal, L., Habener, J.F., 1987. cis-acting DNA sequence controls glucagon gene expression in pancreatic islet cells. Transactions of the Association of American Physicians 100:109-115.

[111] Dumonteil, E., Laser, B., Constant, I., Philippe, J., 1998. Differential regulation of the glucagon and insulin I gene promoters by the basic helix-loophelix transcription factors E47 and BETA2. Journal of Biological Chemistry 273:19945-19954.

[112] Hussain, M.A., Habener, J.F., 1999. Glucagon gene transcription activation mediated by synergistic interactions of pax-6 and cdx-2 with the p300 coactivator. Journal of Biological Chemistry 274:28950-28957.

[113] Jin, T., Trinh, D.K., Wang, F., Drucker, D.J., 1997. The caudal homeobox protein cdx-2/3 activates endogenous proglucagon gene expression in InR1G9 islet cells. Molecular Endocrinology 11:203-209.

[114] Laser, B., Meda, P., Constant, I., Philippe, J., 1996. The caudal-related homeodomain protein $\mathrm{Cdx}-2 / 3$ regulates glucagon gene expression in islet cells. Journal of Biological Chemistry 271:28984-28994.

[115] Philippe, J., Morel, C., Prezioso, V.R., 1994. Glucagon gene expression is negatively regulated by hepatocyte nuclear factor 3 beta. Molecular and Cellular Biology 14:3514-3523.

[116] Ritz-Laser, B., Estreicher, A., Gauthier, B., Philippe, J., 2000. The paired homeodomain transcription factor Pax-2 is expressed in the endocrine pancreas and transactivates the glucagon gene promoter. Journal of Biological Chemistry 275:32708-32715.

[117] Ritz-Laser, B., Estreicher, A., Klages, N., Saule, S., Philippe, J., 1999. Pax-6 and $\mathrm{Cdx}-2 / 3$ interact to activate glucagon gene expression on the G1 control element. Journal of Biological Chemistry 274:4124-4132.

[118] Wang, M., Drucker, D.J., 1995. The LIM domain homeobox gene isl-1 is a positive regulator of islet cell-specific proglucagon gene transcription. Journal of Biological Chemistry 270:12646-12652.

[119] Kaestner, K.H., Katz, J., Liu, Y., Drucker, D.J., Schutz, G., 1999. Inactivation of the winged helix transcription factor HNF3alpha affects glucose homeostasis and islet glucagon gene expression in vivo. Genes and Development 13:495-504.

[120] Lee, C.S., Sund, N.J., Behr, R., Herrera, P.L., Kaestner, K.H., 2005. Foxa2 is required for the differentiation of pancreatic alpha-cells. Developmental Biology 278:484-495.

[121] Sund, N.J., Vatamaniuk, M.Z., Casey, M., Ang, S.L., Magnuson, M.A., Stoffers, D.A., et al., 2001. Tissue-specific deletion of Foxa2 in pancreatic beta cells results in hyperinsulinemic hypoglycemia. Genes and Development 15:1706-1715.

[122] Dong, J., Asa, S.L., Drucker, D.J., 1991. Islet cell and extrapancreatic expression of the LIM domain homeobox gene isl-1. Molecular Endocrinology 5:1633-1641.

[123] Pfaff, S.L., Mendelsohn, M., Stewart, C.L., Edlund, T., Jessell, T.M., 1996. Requirement for LIM homeobox gene Isl1 in motor neuron generation reveals a motor neuron-dependent step in interneuron differentiation. Cell 84:309320. 
[124] Karlsson, O., Thor, S., Norberg, T., Ohlsson, H., Edlund, T., 1990. Insulin gene enhancer binding protein Isl-1 is a member of a novel class of proteins containing both a homeo- and a Cys-His domain. Nature 344: 879-882.

[125] Vallejo, M., Penchuk, L., Habener, J.F., 1992. Somatostatin gene upstream enhancer element activated by a protein complex consisting of CREB, Isl-1like, and alpha-CBF-like transcription factors. Journal of Biological Chemistry 267:12876-12884.

[126] Drucker, D.J., Brubaker, P.L., 1989. Proglucagon gene expression is regulated by a cyclic AMP-dependent pathway in rat intestine. Proceedings of the National Academy of Sciences of the United States of America 86:39533957.

[127] Drucker, D.J., Campos, R., Reynolds, R., Stobie, K., Brubaker, P.L., 1991. The rat glucagon gene is regulated by a protein kinase A-dependent pathway in pancreatic islet cells. Endocrinology 128:394-400.

[128] Knepel, W., Chafitz, J., Habener, J.F., 1990. Transcriptional activation of the rat glucagon gene by the cyclic AMP-responsive element in pancreatic islet cells. Molecular and Cellular Biology 10:6799-6804.

[129] Drucker, D.J., Jin, T., Asa, S.L., Young, T.A., Brubaker, P.L., 1994. Activation of proglucagon gene transcription by protein kinase-A in a novel mouse enteroendocrine cell line. Molecular Endocrinology 8:1646-1655.

[130] Gajic, D., Drucker, D.J., 1993. Multiple cis-acting domains mediate basal and adenosine 3',5'-monophosphate-dependent glucagon gene transcription in a mouse neuroendocrine cell line. Endocrinology 132:1055-1062.

[131] Lu, F., Jin, T., Drucker, D.J., 1996. Proglucagon gene expression is induced by gastrin-releasing peptide in a mouse enteroendocrine cell line. Endocrinology 137:3710-3716.

[132] Ni, Z., Anini, Y., Fang, X., Mills, G., Brubaker, P.L., Jin, T., 2003. Transcriptional activation of the proglucagon gene by lithium and beta-catenin in intestinal endocrine L cells. Journal of Biological Chemistry 278:1380-1387.

[133] Yi, F., Sun, J., Lim, G.E., Fantus, I.G., Brubaker, P.L., Jin, T., 2008. Cross talk between the insulin and Wnt signaling pathways: evidence from intestinal endocrine L cells. Endocrinology 149:2341-2351.

[134] Fang, X., Yu, S.X., Lu, Y., Bast Jr., R.C., Woodgett, J.R., Mills, G.B., 2000. Phosphorylation and inactivation of glycogen synthase kinase 3 by protein kinase A. Proceedings of the National Academy of Sciences of the United States of America 97:11960-11965.

[135] Li, M., Wang, X., Meintzer, M.K., Laessig, T., Birnbaum, M.J., Heidenreich, K.A., 2000. Cyclic AMP promotes neuronal survival by phosphorylation of glycogen synthase kinase 3beta. Molecular and Cellular Biology 20:9356-9363.

[136] Chen, L., Komiya, I., Inman, L., McCorkle, K., Alam, T., Unger, R.H., 1989. Molecular and cellular responses of islets during perturbations of glucose homeostasis determined by in situ hybridization histochemistry. Proceedings of the National Academy of Sciences of the United States of America 86: $1367-1371$.

[137] Philippe, J., 1989. Glucagon gene transcription is negatively regulated by insulin in a hamster islet cell line. Journal of Clinical Investigation 84:672677.

[138] Philippe, J., 1991. Insulin regulation of the glucagon gene is mediated by an insulin-responsive DNA element. Proceedings of the National Academy of Sciences of the United States of America 88:7224-7227.

[139] Gauthier, B.R., Gosmain, Y., Mamin, A., Philippe, J., 2007. The beta-cell specific transcription factor Nkx6.1 inhibits glucagon gene transcription by interfering with Pax6. Biochemical Journal 403:593-601.

[140] Ritz-Laser, B., Estreicher, A., Gauthier, B.R., Mamin, A., Edlund, H., Philippe, J., 2002. The pancreatic beta-cell-specific transcription factor Pax4 inhibits glucagon gene expression through Pax-6. Diabetologia 45:97107.

[141] Fujita, Y., Chui, J.W., King, D.S., Zhang, T., Seufert, J., Pownall, S., et al., 2008. Pax6 and Pdx1 are required for production of glucose-dependent insulinotropic polypeptide in proglucagon-expressing $L$ cells. American Journal of Physiology Endocrinology and Metabolism 295:E648-E657.

[142] Flock, G., Cao, X., Drucker, D.J., 2005. Pdx-1 is not sufficient for repression of proglucagon gene transcription in islet or enteroendocrine cells. Endocrinology 146:441-449.

[143] Yi, F., Brubaker, P.L., Jin, T., 2005. TCF-4 mediates cell type-specific regulation of proglucagon gene expression by beta-catenin and glycogen synthase kinase-3beta. Journal of Biological Chemistry 280:1457-1464.

[144] Larraufie, P., Roberts, G.P., McGavigan, A.K., Kay, R.G., Li, J., Leiter, A., et al., 2019. Important role of the GLP-1 Axis for glucose homeostasis after bariatric surgery. Cell Reports 26:1399-1408 e1396.

[145] Roberts, G.P., Kay, R.G., Howard, J., Hardwick, R.H., Reimann, F., Gribble, F.M., 2018. Gastrectomy with Roux-en-Y reconstruction as a lean model of bariatric surgery. Surgery for Obesity and Related Diseases 14: 562-568.

[146] Roberts, G.P., Larraufie, P., Richards, P., Kay, R.G., Galvin, S.G. Miedzybrodzka, E.L., et al., 2019. Comparison of human and murine enteroendocrine cells by transcriptomic and peptidomic profiling. Diabetes 68:1062-1072.

[147] Baggio, L.L., Drucker, D.J., 2007. Biology of incretins: GLP-1 and GIP. Gastroenterology 132:2131-2157.

[148] Larsen, P.J., Tang-Christensen, M., Holst, J.J., Orskov, C., 1997. Distribution of glucagon-like peptide-1 and other preproglucagon-derived peptides in the rat hypothalamus and brainstem. Neuroscience 77:257-270.

[149] Tucker, J.D., Dhanvantari, S., Brubaker, P.L., 1996. Proglucagon processing in islet and intestinal cell lines. Regulatory Peptides 62:29-35.

[150] Vrang, N., Hansen, M., Larsen, P.J., Tang-Christensen, M., 2007. Characterization of brainstem preproglucagon projections to the paraventricular and dorsomedial hypothalamic nuclei. Brain Research 1149:118-126.

[151] Rouille, Y., Martin, S., Steiner, D.F., 1995. Differential processing of proglucagon by the subtilisin-like prohormone convertases PC2 and PC3 to generate either glucagon or glucagon-like peptide. Journal of Biological Chemistry 270:26488-26496.

[152] Holst, J.J., 2007. The physiology of glucagon-like peptide 1. Physiological Reviews 87:1409-1439.

[153] Holst, J.J., Bersani, M., Johnsen, A.H., Kofod, H., Hartmann, B., Orskov, C., 1994. Proglucagon processing in porcine and human pancreas. Journal of Biological Chemistry 269:18827-18833.

[154] Furuta, M., Yano, H., Zhou, A., Rouille, Y., Holst, J.J., Carroll, R., et al., 1997. Defective prohormone processing and altered pancreatic islet morphology in mice lacking active SPC2. Proceedings of the National Academy of Sciences of the United States of America 94:6646-6651.

[155] Furuta, M., Zhou, A., Webb, G., Carroll, R., Ravazzola, M., Orci, L., et al., 2001. Severe defect in proglucagon processing in islet A-cells of prohormone convertase 2 null mice. Journal of Biological Chemistry 276:27197-27202.

[156] Webb, G.C., Akbar, M.S., Zhao, C., Swift, H.H., Steiner, D.F., 2002. Glucagon replacement via micro-osmotic pump corrects hypoglycemia and alpha-cell hyperplasia in prohormone convertase 2 knockout mice. Diabetes 51: 398-405.

[157] Kilimnik, G., Kim, A., Steiner, D.F., Friedman, T.C., Hara, M., 2010. Intraislet production of GLP-1 by activation of prohormone convertase $1 / 3$ in pancreatic alpha-cells in mouse models of ss-cell regeneration. Islets 2:149-155.

[158] Nie, Y., Nakashima, M., Brubaker, P.L., Li, Q.L., Perfetti, R., Jansen, E., et al., 2000. Regulation of pancreatic PC1 and PC2 associated with increased glucagon-like peptide 1 in diabetic rats. Journal of Clinical Investigation 105: 955-965.

[159] Thyssen, S., Arany, E., Hill, D.J., 2006. Ontogeny of regeneration of beta-cells in the neonatal rat after treatment with streptozotocin. Endocrinology 147: 2346-2356.

[160] Wilson, M.E., Kalamaras, J.A., German, M.S., 2002. Expression pattern of IAPP and prohormone convertase $1 / 3$ reveals a distinctive set of endocrine 
cells in the embryonic pancreas. Mechanisms of Development 115:171176.

[161] McGirr, R., Ejbick, C.E., Carter, D.E., Andrews, J.D., Nie, Y., Friedman, T.C., et al., 2005. Glucose dependence of the regulated secretory pathway in alphaTC1-6 cells. Endocrinology 146:4514-4523.

[162] Whalley, N.M., Pritchard, L.E., Smith, D.M., White, A., 2011. Processing of proglucagon to GLP-1 in pancreatic alpha-cells: is this a paracrine mechanism enabling GLP-1 to act on beta-cells? Journal of Endocrinology 211:99106.

[163] Ellingsgaard, H., Ehses, J.A., Hammar, E.B., Van Lommel, L., Quintens, R., Martens, G., et al., 2008. Interleukin-6 regulates pancreatic alpha-cell mass expansion. Proceedings of the National Academy of Sciences of the United States of America 105:13163-13168.

[164] Ellingsgaard, H., Hauselmann, I., Schuler, B., Habib, A.M., Baggio, L.L., Meier, D.T., et al., 2011. Interleukin-6 enhances insulin secretion by increasing glucagon-like peptide- 1 secretion from $L$ cells and alpha cells. Nature Medicine 17:1481-1489.

[165] Wideman, R.D., Yu, I.L., Webber, T.D., Verchere, C.B., Johnson, J.D., Cheung, A.T., et al., 2006. Improving function and survival of pancreatic islets by endogenous production of glucagon-like peptide 1 (GLP-1). Proceedings of the National Academy of Sciences of the United States of America 103:13468-13473.

[166] Jun, L.S., Millican, R.L., Hawkins, E.D., Konkol, D.L., Showalter, A.D., Christe, M.E., et al., 2015. Absence of glucagon and insulin action reveals a role for the GLP-1 receptor in endogenous glucose production. Diabetes 64 : 819-827.

[167] Omar, B.A., Andersen, B., Hald, J., Raun, K., Nishimura, E., Ahren, B., 2014. Fibroblast growth factor 21 (FGF21) and glucagon-like peptide 1 contribute to diabetes resistance in glucagon receptor-deficient mice. Diabetes 63:101110.

[168] Moffett, R.C., Vasu, S., Thorens, B., Drucker, D.J., Flatt, P.R., 2014. Incretin receptor null mice reveal key role of GLP-1 but not GIP in pancreatic beta cell adaptation to pregnancy. PLoS One 9:e96863.

[169] Vasu, S., Moffett, R.C., Thorens, B., Flatt, P.R., 2014. Role of endogenous GLP-1 and GIP in beta cell compensatory responses to insulin resistance and cellular stress. PLoS One 9:e101005.

[170] Chambers, A.P., Sorrell, J.E., Haller, A., Roelofs, K., Hutch, C.R., Kim, K.S., et al., 2017. The role of pancreatic preproglucagon in glucose homeostasis in mice. Cell Metabolism 25:927-934 e923.

[171] Lin, H.V., Wang, J., Wang, J., Li, W., Wang, X., Alston, J.T., et al., 2018. GPR142 prompts glucagon-like Peptide-1 release from islets to improve beta cell function. Molecular Metabolism 11:205-211.

[172] Traub, S., Meier, D.T., Schulze, F., Dror, E., Nordmann, T.M., Goetz, N., et al., 2017. Pancreatic alpha cell-derived glucagon-related peptides are required for beta cell adaptation and glucose homeostasis. Cell Reports 18:31923203.

[173] Capozzi, M.E., Svendsen, B., Encisco, S.E., Lewandowski, S.L., Martin, M.D., Lin, H., et al., 2019. Beta Cell tone is defined by proglucagon peptides through CAMP signaling. JCl Insight 4.

[174] Orskov, C., Bersani, M., Johnsen, A.H., Hojrup, P., Holst, J.J., 1989. Complete sequences of glucagon-like peptide-1 from human and pig small intestine. Journal of Biological Chemistry 264:12826-12829.

[175] Orskov, C., Rabenhoj, L., Wettergren, A., Kofod, H., Holst, J.J., 1994. Tissue and plasma concentrations of amidated and glycine-extended glucagon-like peptide I in humans. Diabetes 43:535-539.

[176] Hansen, L., Hartmann, B., Bisgaard, T., Mineo, H., Jorgensen, P.N., Holst, J.J., 2000. Somatostatin restrains the secretion of glucagon-like peptide- 1 and -2 from isolated perfused porcine ileum. American Journal of Physiology Endocrinology and Metabolism 278:E1010-E1018.
[177] Mojsov, S., Kopczynski, M.G., Habener, J.F., 1990. Both amidated and nonamidated forms of glucagon-like peptide I are synthesized in the rat intestine and the pancreas. Journal of Biological Chemistry 265:8001-8008.

[178] Kuhre, R.E., Albrechtsen, N.W., Windelov, J.A., Svendsen, B., Hartmann, B., Holst, J.J., 2014. GLP-1 amidation efficiency along the length of the intestine in mice, rats and pigs and in GLP-1 secreting cell lines. Peptides 55:52-57.

[179] Philippe, J., Mojsov, S., Drucker, D.J., Habener, J.F., 1986. Proglucagon processing in a rat islet cell line resembles phenotype of intestine rather than pancreas. Endocrinology 119:2833-2839.

[180] Drucker, D.J., Mojsov, S., Habener, J.F., 1986. Cell-specific posttranslational processing of preproglucagon expressed from a metallothionein-glucagon fusion gene. Journal of Biological Chemistry 261: 9637-9643.

[181] Orskov, C., Wettergren, A., Holst, J.J., 1993. Biological effects and metabolic rates of glucagonlike peptide-1 7-36 amide and glucagonlike peptide-1 7-37 in healthy subjects are indistinguishable. Diabetes 42:658-661.

[182] Hui, H., Farilla, L., Merkel, P., Perfetti, R., 2002. The short half-life of glucagon-like peptide- 1 in plasma does not reflect its long-lasting beneficial effects. European Journal of Endocrinology 146:863-869.

[183] Deacon, C.F., Pridal, L., Klarskov, L., Olesen, M., Holst, J.J., 1996. Glucagonlike peptide 1 undergoes differential tissue-specific metabolism in the anesthetized pig. American Journal of Physiology 271:E458-E464.

[184] Meier, J.J., Nauck, M.A., Kranz, D., Holst, J.J., Deacon, C.F., Gaeckler, D., et al., 2004. Secretion, degradation, and elimination of glucagon-like peptide 1 and gastric inhibitory polypeptide in patients with chronic renal insufficiency and healthy control subjects. Diabetes 53:654-662.

[185] Deacon, C.F., Johnsen, A.H., Holst, J.J., 1995. Degradation of glucagon-like peptide- 1 by human plasma in vitro yields an $\mathrm{N}$-terminally truncated peptide that is a major endogenous metabolite in vivo. The Journal of Cinical Endocrinology and Metabolism 80:952-957.

[186] Kieffer, T.J., Mclntosh, C.H., Pederson, R.A., 1995. Degradation of glucosedependent insulinotropic polypeptide and truncated glucagon-like peptide 1 in vitro and in vivo by dipeptidyl peptidase IV. Endocrinology 136:3585-3596.

[187] Mentlein, R., Gallwitz, B., Schmidt, W.E., 1993. Dipeptidyl-peptidase IV hydrolyses gastric inhibitory polypeptide, glucagon-like peptide-1(7-36)amide, peptide histidine methionine and is responsible for their degradation in human serum. European Journal of Biochemistry 214:829-835.

[188] De Meester, I., Korom, S., Van Damme, J., Scharpe, S., 1999. CD26, let it cut or cut it down. Immunology Today 20:367-375.

[189] Windelov, J.A., Wewer Albrechtsen, N.J., Kuhre, R.E., Jepsen, S.L., Hornburg, D., Pedersen, J., et al., 2017. Why is it so difficult to measure glucagon-like peptide-1 in a mouse? Diabetologia 60:2066-2075.

[190] Drucker, D.J., 2007. Dipeptidyl peptidase-4 inhibition and the treatment of type 2 diabetes: preclinical biology and mechanisms of action. Diabetes Care 30:1335-1343.

[191] Hansen, L., Deacon, C.F., Orskov, C., Holst, J.J., 1999. Glucagon-like peptide-1-(7-36)amide is transformed to glucagon-like peptide-1-(9-36) amide by dipeptidyl peptidase IV in the capillaries supplying the $L$ cells of the porcine intestine. Endocrinology 140:5356-5363.

[192] Holst, J.J., Deacon, C.F., 1998. Inhibition of the activity of dipeptidylpeptidase IV as a treatment for type 2 diabetes. Diabetes 47:1663-1670.

[193] Deacon, C.F., Hughes, T.E., Holst, J.J., 1998. Dipeptidyl peptidase IV inhibition potentiates the insulinotropic effect of glucagon-like peptide 1 in the anesthetized pig. Diabetes 47:764-769.

[194] Parkes, D., Jodka, C., Smith, P., Nayak, S., Rinehart, L., Gingerich, R., et al., 2001. Pharmacokinetic actions of exendin-4 in the rat:comparison with glucagon-like peptide-1. Drug Development Research 53:260-267.

[195] Nauck, M.A., 1998. Glucagon-like peptide 1 (GLP-1): a potent gut hormone with a possible therapeutic perspective. Acta Diabetologica 35:117-129. 
[196] Vahl, T.P., Paty, B.W., Fuller, B.D., Prigeon, R.L., D’Alessio, D.A., 2003. Effects of GLP-1-(7-36)NH2, GLP-1-(7-37), and GLP-1- (9-36)NH2 on intravenous glucose tolerance and glucose-induced insulin secretion in healthy humans. The Journal of Cinical Endocrinology and Metabolism 88:17721779.

[197] Zander, M., Madsbad, S., Deacon, C.F., Holst, J.J., 2006. The metabolite generated by dipeptidyl-peptidase 4 metabolism of glucagon-like peptide-1 has no influence on plasma glucose levels in patients with type 2 diabetes. Diabetologia 49:369-374.

[198] Elahi, D., Egan, J.M., Shannon, R.P., Meneilly, G.S., Khatri, A., Habener, J.F., et al., 2008. GLP-1 (9-36) amide, cleavage product of GLP-1 (7-36) amide, is a glucoregulatory peptide. Obesity (Silver Spring) 16:1501-1509.

[199] Deacon, C.F., Plamboeck, A., Moller, S., Holst, J.J., 2002. GLP-1-(9-36) amide reduces blood glucose in anesthetized pigs by a mechanism that does not involve insulin secretion. American Journal of Physiology Endocrinology and Metabolism 282:E873-E879.

[200] Meier, J.J., Gethmann, A., Nauck, M.A., Gotze, 0., Schmitz, F., Deacon, C.F., et al., 2006. The glucagon-like peptide-1 metabolite GLP-1-(9-36) amide reduces postprandial glycemia independently of gastric emptying and insulin secretion in humans. American Journal of Physiology Endocrinology and Metabolism 290:E1118-E1123.

[201] Ban, K., Noyan-Ashraf, M.H., Hoefer, J., Bolz, S.S., Drucker, D.J., Husain, M., 2008. Cardioprotective and vasodilatory actions of glucagon-like peptide 1 receptor are mediated through both glucagon-like peptide 1 receptordependent and -independent pathways. Circulation 117:2340-2350.

[202] Parker, J.C., Lavery, K.S., Irwin, N., Green, B.D., Greer, B., Harriott, P., et al., 2006. Effects of sub-chronic exposure to naturally occurring $\mathrm{N}$-terminally truncated metabolites of glucose-dependent insulinotrophic polypeptide (GIP) and glucagon-like peptide-1 (GLP-1), GIP(3-42) and GLP-1(9-36)amide, on insulin secretion and glucose homeostasis in ob/ob mice. Journal of Endocrinology 191:93-100.

[203] Porter, D., Faivre, E., Flatt, P.R., Holscher, C., Gault, V.A., 2012. Actions of incretin metabolites on locomotor activity, cognitive function and in vivo hippocampal synaptic plasticity in high fat fed mice. Peptides 35:1-8.

[204] Knudsen, L.B., Pridal, L., 1996. Glucagon-like peptide-1-(9-36) amide is a major metabolite of glucagon-like peptide-1-(7-36) amide after in vivo administration to dogs, and it acts as an antagonist on the pancreatic receptor. European Journal of Pharmacology 318:429-435.

[205] Bryant, M.G., Bloom, S.R., Polak, J.M., Hobbs, S., Domschke, W., Domschke, S., et al., 1983. Measurement of gut hormonal peptides in biopsies from human stomach and proximal small intestine. Gut 24:114-119.

[206] Damholt, A.B., Kofod, H., Buchan, A.M., 1999. Immunocytochemical evidence for a paracrine interaction between GIP and GLP-1-producing cells in canine small intestine. Cell and Tissue Research 298:287-293.

[207] Drucker, D.J., 2006. The biology of incretin hormones. Cell Metabolism 3: 153-165.

[208] Eissele, R., Goke, R., Willemer, S., Harthus, H.P., Vermeer, H., Arnold, R., et al., 1992. Glucagon-like peptide-1 cells in the gastrointestinal tract and pancreas of rat, pig and man. European Journal of Clinical Investigation 22: 283-291.

[209] Larsson, L.I., Holst, J., Hakanson, R., Sundler, F., 1975. Distribution and properties of glucagon immunoreactivity in the digestive tract of various mammals: an immunohistochemical and immunochemical study. Histochemistry 44:281-290.

[210] Layer, P., Holst, J.J., Grandt, D., Goebell, H., 1995. Ileal release of glucagonlike peptide-1 (GLP-1). Association with inhibition of gastric acid secretion in humans. Digestive Diseases and Sciences 40:1074-1082.

[211] Caiazzo, R., Branche, J., Daoudi, M., Solecki, G., Hubert, T., Quenon, A., et al., 2018. Increased postprandial glucagon-like peptide-1 (GLP-1) production after endoscopic gastrointestinal bypass using the Cousin lumenapposing stent in a porcine model. Endoscopy 50:14-21.
[212] Chambers, A.P., Jessen, L., Ryan, K.K., Sisley, S., Wilson-Perez, H.E., Stefater, M.A., et al., 2011. Weight-independent changes in blood glucose homeostasis after gastric bypass or vertical sleeve gastrectomy in rats. Gastroenterology 141:950-958.

[213] Chambers, A.P., Stefater, M.A., Wilson-Perez, H.E., Jessen, L., Sisley, S., Ryan, K.K., et al., 2011. Similar effects of roux-en-Y gastric bypass and vertical sleeve gastrectomy on glucose regulation in rats. Physiology and Behavior 105:120-123.

[214] Cummings, D.E., Overduin, J., Shannon, M.H., Foster-Schubert, K.E., Conference, A.B.S.C., 2005. Hormonal mechanisms of weight loss and diabetes resolution after bariatric surgery. Surgery for Obesity and Related Diseases 1:358-368.

[215] Peterli, R., Wolnerhanssen, B., Peters, T., Devaux, N., Kern, B., ChristoffelCourtin, C., et al., 2009. Improvement in glucose metabolism after bariatric surgery: comparison of laparoscopic Roux-en- $Y$ gastric bypass and laparoscopic sleeve gastrectomy: a prospective randomized trial. Annals of Surgery 250:234-241.

[216] Verhaeghe, R., Zerrweck, C., Hubert, T., Trechot, B., Gmyr, V., D'Herbomez, M., et al., 2014. Gastric bypass increases postprandial insulin and GLP-1 in nonobese minipigs. European Surgical Research 52:41-49.

[217] Qualmann, C., Nauck, M.A., Holst, J.J., Orskov, C., Creutzfeldt, W., 1995. Glucagon-like peptide 1 (7-36 amide) secretion in response to luminal sucrose from the upper and lower gut. A study using alpha-glucosidase inhibition (acarbose). Scandinavian Journal of Gastroenterology 30:892896.

[218] Seifarth, C., Bergmann, J., Holst, J.J., Ritzel, R., Schmiegel, W., Nauck, M.A., 1998. Prolonged and enhanced secretion of glucagon-like peptide 1 (7-36 amide) after oral sucrose due to alpha-glucosidase inhibition (acarbose) in Type 2 diabetic patients. Diabetic Medicine 15:485-491.

[219] Craig, C.M., Liu, L.F., Deacon, C.F., Holst, J.J., McLaughlin, T.L., 2017. Critical role for GLP-1 in symptomatic post-bariatric hypoglycaemia. Diabetologia 60:531-540.

[220] Wilson-Perez, H.E., Chambers, A.P., Ryan, K.K., Li, B., Sandoval, D.A., Stoffers, D., et al., 2013. Vertical sleeve gastrectomy is effective in two genetic mouse models of glucagon-like Peptide 1 receptor deficiency. Diabetes 62:2380-2385.

[221] Lutz, T.A., 2018. Considering our methods: methodological issues with rodent models of appetite and obesity research. Physiology \& Behavior 192: $182-187$.

[222] Svane, M.S., Bojsen-Moller, K.N., Martinussen, C., Dirksen, C., Madsen, J.L., Reitelseder, S., et al., 2019. Postprandial nutrient handling and gastrointestinal secretion of hormones after Roux-en-Y gastric bypass vs sleeve gastrectomy. Gastroenterology.

[223] Ye, J., Hao, Z., Mumphrey, M.B., Townsend, R.L., Patterson, L.M., Stylopoulos, N., et al., 2014. GLP-1 receptor signaling is not required for reduced body weight after RYGB in rodents. American Journal of Physiology Regulatory Integrative and Comparative Physiology 306:R352-R362.

[224] Svane, M.S., Jorgensen, N.B., Bojsen-Moller, K.N., Dirksen, C., Nielsen, S. Kristiansen, V.B., et al., 2016. Peptide YY and glucagon-like peptide-1 contribute to decreased food intake after Roux-en-Y gastric bypass surgery. International Journal of Obesity (London).

[225] Ten Kulve, J.S., Veltman, D.J., Gerdes, V.E.A., van Bloemendaal, L., Barkhof, F., Deacon, C.F., et al., 2017. Elevated postoperative endogenous GLP-1 levels mediate effects of Roux-en-Y gastric bypass on neural responsivity to food cues. Diabetes Care 40:1522-1529.

[226] Holst, J.J., Madsbad, S., Bojsen-Moller, K.N., Svane, M.S., Jorgensen, N.B., Dirksen, C., et al., 2018. Mechanisms in bariatric surgery: gut hormones, diabetes resolution, and weight loss. Surgery for Obesity and Related Diseases 14:708-714.

[227] Fujii, Y., Osaki, N., Hase, T., Shimotoyodome, A., 2015. Ingestion of coffee polyphenols increases postprandial release of the active glucagon-like 
peptide-1 (GLP-1(7-36)) amide in C57BL/6J mice. Journal of Nutrition Sciences 4:e9.

[228] Higuchi, N., Hira, T., Yamada, N., Hara, H., 2013. Oral administration of corn zein hydrolysate stimulates GLP-1 and GIP secretion and improves glucose tolerance in male normal rats and Goto-Kakizaki rats. Endocrinology 154: 3089-3098.

[229] Jejelava, N., Kaufman, S., Krieger, J.P., Terra, M.M., Langhans, W. Arnold, M., 2018. Intestinal lymph as a readout of meal-induced GLP-1 release in an unrestrained rat model. American Journal of Physiology Regulatory Integrative and Comparative Physiology.

[230] Moghadam, A.A., Moran, T.H., Dailey, M.J., 2017. Alterations in circadian and meal-induced gut peptide levels in lean and obese rats. Experimental Biology and Medicine (Maywood) 242:1786-1794.

[231] Nakajima, S., Hira, T., Hara, H., 2015. Postprandial glucagon-like peptide-1 secretion is increased during the progression of glucose intolerance and obesity in high-fat/high-sucrose diet-fed rats. British Journal of Nutrition 113: $1477-1488$.

[232] Rocca, A.S., LaGreca, J., Kalitsky, J., Brubaker, P.L., 2001. Monounsaturated fatty acid diets improve glycemic tolerance through increased secretion of glucagon-like peptide-1. Endocrinology 142:1148-1155.

[233] Deshpande, M.C., Venkateswarlu, V., Mantri, A.H., Gadewar, S.T., 2011. Targeting enteral endocrinal L-cells with dietary carbohydrates, by increasing the availability of miglitol in the intestinal lumen, leads to multi-fold enhancement of plasma glucagon-like peptide-1 levels in non-diabetic canines. Drug Development and Industrial Pharmacy 37:506-517.

[234] Ionut, V., Liberty, I.F., Hucking, K., Lottati, M., Stefanovski, D., Zheng, D., et al., 2006. Exogenously imposed postprandial-like rises in systemic glucose and GLP-1 do not produce an incretin effect, suggesting an indirect mechanism of GLP-1 action. American Journal of Physiology Endocrinology and Metabolism 291:E779-E785.

[235] Lubbs, D.C., Vester Boler, B.M., Ridge, T.K., Spears, J.K., Graves, T.K., Swanson, K.S., 2010. Dietary macronutrients and feeding frequency affect fasting and postprandial concentrations of hormones involved in appetite regulation in adult dogs. Journal of Animal Science 88:3945-3953.

[236] Orskov, C., Wettergren, A., Holst, J.J., 1996. Secretion of the incretin hormones glucagon-like peptide-1 and gastric inhibitory polypeptide correlates with insulin secretion in normal man throughout the day. Scandinavian Journal of Gastroenterology 31:665-670.

[237] Vilsboll, T., Krarup, T., Sonne, J., Madsbad, S., Volund, A., Juul, A.G., et al., 2003. Incretin secretion in relation to meal size and body weight in healthy subjects and people with type 1 and type 2 diabetes mellitus. The Journal of Cinical Endocrinology and Metabolism 88:2706-2713.

[238] Elliott, R.M., Morgan, L.M., Tredger, J.A., Deacon, S., Wright, J., Marks, V., 1993. Glucagon-like peptide-1 (7-36)amide and glucose-dependent insulinotropic polypeptide secretion in response to nutrient ingestion in man: acute post-prandial and 24-h secretion patterns. Journal of Endocrinology 138: $159-166$.

[239] Herrmann, C., Goke, R., Richter, G., Fehmann, H.C., Arnold, R., Goke, B., 1995. Glucagon-like peptide-1 and glucose-dependent insulin-releasing polypeptide plasma levels in response to nutrients. Digestion 56:117-126.

[240] Kuhre, R.E., Wewer Albrechtsen, N.J., Hartmann, B., Deacon, C.F., Holst, J.J., 2015. Measurement of the incretin hormones: glucagon-like peptide-1 and glucose-dependent insulinotropic peptide. Journal of Diabetic Complications 29:445-450.

[241] Hjollund, K.R., Deacon, C.F., Holst, J.J., 2011. Dipeptidyl peptidase-4 inhibition increases portal concentrations of intact glucagon-like peptide-1 (GLP1) to a greater extent than peripheral concentrations in anaesthetised pigs. Diabetologia 54:2206-2208.

[242] Holst, J.J., Deacon, C.F., 2005. Glucagon-like peptide-1 mediates the therapeutic actions of DPP-IV inhibitors. Diabetologia 48:612-615.
[243] Toft-Nielson, M., Madsbad, S., Holst, J.J., 1996. The effect of glucagon-like peptide I (GLP-I) on glucose elimination in healthy subjects depends on the pancreatic glucoregulatory hormones. Diabetes 45:552-556.

[244] Dailey, M.J., Stingl, K.C., Moran, T.H., 2012. Disassociation between preprandial gut peptide release and food-anticipatory activity. Endocrinology 153:132-142.

[245] Vahl, T.P., Drazen, D.L., Seeley, R.J., D’Alessio, D.A., Woods, S.C., 2010. Meal-anticipatory glucagon-like peptide-1 secretion in rats. Endocrinology 151:569-575.

[246] Balks, H.J., Holst, J.J., von zur Muhlen, A., Brabant, G., 1997. Rapid oscillations in plasma glucagon-like peptide-1 (GLP-1) in humans: cholinergic control of GLP-1 secretion via muscarinic receptors. The Journal of Cinical Endocrinology and Metabolism 82:786-790.

[247] Gribble, F.M., Williams, L., Simpson, A.K., Reimann, F., 2003. A novel glucose-sensing mechanism contributing to glucagon-like peptide-1 secretion from the GLUTag cell line. Diabetes 52:1147-1154.

[248] Sugiyama, K., Manaka, H., Kato, T., Yamatani, K., Tominaga, M., Sasaki, H., 1994. Stimulation of truncated glucagon-like peptide-1 release from the isolated perfused canine ileum by glucose absorption. Digestion 55:24-28.

[249] Kuhre, R.E., Gribble, F.M., Hartmann, B., Reimann, F., Windelov, J.A., Rehfeld, J.F., et al., 2014. Fructose stimulates GLP-1 but not GIP secretion in mice, rats, and humans. American Journal of Physiology Gastrointestinal and Liver Physiology 306:G622-G630.

[250] Dumoulin, V., Moro, F., Barcelo, A., Dakka, T., Cuber, J.C., 1998. Peptide YY, glucagon-like peptide-1, and neurotensin responses to luminal factors in the isolated vascularly perfused rat ileum. Endocrinology 139:3780-3786.

[251] Hirasawa, A., Tsumaya, K., Awaji, T., Katsuma, S., Adachi, T., Yamada, M., et al., 2005. Free fatty acids regulate gut incretin glucagon-like peptide-1 secretion through GPR120. Nature Medicine 11:90-94.

[252] Christensen, L.W., Kuhre, R.E., Janus, C., Svendsen, B., Holst, J.J., 2015. Vascular, but not luminal, activation of FFAR1 (GPR40) stimulates GLP-1 secretion from isolated perfused rat small intestine. Physics Reports 3.

[253] Reimer, R.A., Darimont, C., Gremlich, S., Nicolas-Metral, V., Ruegg, U.T., Mace, K., 2001. A human cellular model for studying the regulation of glucagon-like peptide-1 secretion. Endocrinology 142:4522-4528.

[254] Gunnarsson, P.T., Winzell, M.S., Deacon, C.F., Larsen, M.0., Jelic, K., Carr, R.D., et al., 2006. Glucose-induced incretin hormone release and inactivation are differently modulated by oral fat and protein in mice. Endocrinology 147:3173-3180.

[255] Hall, W.L., Millward, D.J., Long, S.J., Morgan, L.M., 2003. Casein and whey exert different effects on plasma amino acid profiles, gastrointestinal hormone secretion and appetite. British Journal of Nutrition 89:239-248.

[256] Hira, T., Mochida, T., Miyashita, K., Hara, H., 2009. GLP-1 secretion is enhanced directly in the ileum but indirectly in the duodenum by a newly identified potent stimulator, zein hydrolysate, in rats. American Journal of Physiology Gastrointestinal and Liver Physiology 297:G663-G671.

[257] Mochida, T., Hira, T., Hara, H., 2010. The corn protein, zein hydrolysate, administered into the ileum attenuates hyperglycemia via its dual action on glucagon-like peptide-1 secretion and dipeptidyl peptidase-IV activity in rats. Endocrinology 151:3095-3104.

[258] Gameiro, A., Reimann, F., Habib, A.M., O'Malley, D., Williams, L., Simpson, A.K., et al., 2005. The neurotransmitters glycine and GABA stimulate glucagon-like peptide-1 release from the GLUTag cell line. Journal of Physiology 569:761-772.

[259] Reimann, F., Williams, L., da Silva Xavier, G., Rutter, G.A., Gribble, F.M., 2004. Glutamine potently stimulates glucagon-like peptide-1 secretion from GLUTag cells. Diabetologia 47:1592-1601.

[260] Tolhurst, G., Zheng, Y., Parker, H.E., Habib, A.M., Reimann, F., Gribble, F.M., 2011. Glutamine triggers and potentiates glucagon-like peptide-1 secretion by raising cytosolic $\mathrm{Ca}^{2+}$ and cAMP. Endocrinology 152:405-413. 
[261] Punjabi, M., Arnold, M., Ruttimann, E., Graber, M., Geary, N., PachecoLopez, G., et al., 2014. Circulating glucagon-like peptide-1 (GLP-1) inhibits eating in male rats by acting in the hindbrain and without inducing avoidance. Endocrinology 155:1690-1699.

[262] Faerch, K., Torekov, S.S., Vistisen, D., Johansen, N.B., Witte, D.R., Jonsson, A., et al., 2015. GLP-1 response to oral glucose is reduced in prediabetes, screen-detected type 2 diabetes, and obesity and influenced by sex: the ADDITION-PRO study. Diabetes 64:2513-2525.

[263] Calanna, S., Christensen, M., Holst, J.J., Laferrere, B., Gluud, L.L., Vilsboll, T., et al., 2013. Secretion of glucagon-like peptide- 1 in patients with type 2 diabetes mellitus: systematic review and meta-analyses of clinical studies. Diabetologia 56:965-972.

[264] Matikainen, N., Bogl, L.H., Hakkarainen, A., Lundbom, J., Lundbom, N., Kaprio, J., et al., 2014. GLP-1 responses are heritable and blunted in acquired obesity with high liver fat and insulin resistance. Diabetes Care 37: $242-251$.

[265] Reimann, F., Gribble, F.M., 2002. Glucose-sensing in glucagon-like peptide1-secreting cells. Diabetes 51:2757-2763.

[266] Tolhurst, G., Reimann, F., Gribble, F.M., 2009. Nutritional regulation of glucagon-like peptide-1 secretion. Journal of Physiology 587:27-32.

[267] Ashcroft, F.M., Gribble, F.M., 1999. ATP-sensitive K+ channels and insulin secretion: their role in health and disease. Diabetologia 42:903-919.

[268] Ashcroft, F.M., Rorsman, P., 2013. K(ATP) channels and islet hormone secretion: new insights and controversies. Nature Reviews Endocrinology 9: $660-669$.

[269] Gribble, F.M., Reimann, F., 2003. Sulphonylurea action revisited: the postcloning era. Diabetologia 46:875-891.

[270] Henquin, J.C., 1992. The fiftieth anniversary of hypoglycaemic sulphonamides. How did the mother compound work? Diabetologia 35:907-912.

[271] Nielsen, L.B., Ploug, K.B., Swift, P., Orskov, C., Jansen-Olesen, I., Chiarelli, F., et al., 2007. Co-localisation of the Kir6.2/SUR1 channel complex with glucagon-like peptide-1 and glucose-dependent insulinotrophic polypeptide expression in human ileal cells and implications for glycaemic control in new onset type 1 diabetes. European Journal of Endocrinology 156:663671.

[272] Cani, P.D., Holst, J.J., Drucker, D.J., Delzenne, N.M., Thorens, B., Burcelin, R., et al., 2007. GLUT2 and the incretin receptors are involved in glucose-induced incretin secretion. Molecular and Cellular Endocrinology 276:18-23.

[273] Parker, H.E., Adriaenssens, A., Rogers, G., Richards, P., Koepsell, H., Reimann, F., et al., 2012. Predominant role of active versus facilitative glucose transport for glucagon-like peptide- 1 secretion. Diabetologia 55 : $2445-2455$.

[274] Gorboulev, V., Schurmann, A., Vallon, V., Kipp, H., Jaschke, A., Klessen, D., et al., 2012. $\mathrm{Na}(+)$-D-glucose cotransporter SGLT1 is pivotal for intestinal glucose absorption and glucose-dependent incretin secretion. Diabetes 61 : 187-196.

[275] Moriya, R., Shirakura, T., Ito, J., Mashiko, S., Seo, T., 2009. Activation of sodium-glucose cotransporter 1 ameliorates hyperglycemia by mediating incretin secretion in mice. American Journal of Physiology Endocrinology and Metabolism 297:E1358-E1365.

[276] Mace, 0.J., Schindler, M., Patel, S., 2012. The regulation of K- and L-cell activity by GLUT2 and the calcium-sensing receptor CasR in rat small intestine. Journal of Physiology 590:2917-2936.

[277] Ezcurra, M., Reimann, F., Gribble, F.M., Emery, E., 2013. Molecular mechanisms of incretin hormone secretion. Current Opinion in Pharmacology 13: 922-927.

[278] Reimann, F., Gribble, F.M., 2016. Mechanisms underlying glucosedependent insulinotropic polypeptide and glucagon-like peptide-1 secretion. Journal of Diabetes Investigation 7(Suppl. 1):13-19.
[279] Steinert, R.E., Frey, F., Topfer, A., Drewe, J., Beglinger, C., 2011. Effects of carbohydrate sugars and artificial sweeteners on appetite and the secretion of gastrointestinal satiety peptides. British Journal of Nutrition 105:13201328.

[280] Jang, H.J., Kokrashvili, Z., Theodorakis, M.J., Carlson, O.D., Kim, B.J., Zhou, J., et al., 2007. Gut-expressed gustducin and taste receptors regulate secretion of glucagon-like peptide-1. Proceedings of the National Academy of Sciences of the United States of America 104:15069-15074.

[281] Reimann, F., Habib, A.M., Tolhurst, G., Parker, H.E., Rogers, G.J., Gribble, F.M., 2008. Glucose sensing in L cells: a primary cell study. Cell Metabolism 8:532-539.

[282] Saltiel, M.Y., Kuhre, R.E., Christiansen, C.B., Eliasen, R., CondeFrieboes, K.W., Rosenkilde, M.M., et al., 2017. Sweet taste receptor activation in the gut is of limited importance for glucose-stimulated GLP-1 and GIP secretion. Nutrients 9 .

[283] Ma, J., Chang, J., Checklin, H.L., Young, R.L., Jones, K.L., Horowitz, M., et al., 2010. Effect of the artificial sweetener, sucralose, on small intestinal glucose absorption in healthy human subjects. British Journal of Nutrition 104:803-806.

[284] Maersk, M., Belza, A., Holst, J.J., Fenger-Gron, M., Pedersen, S.B., Astrup, A., et al., 2012. Satiety scores and satiety hormone response after sucrose-sweetened soft drink compared with isocaloric semi-skimmed milk and with non-caloric soft drink: a controlled trial. European Journal of Clinical Nutrition 66:523-529.

[285] Husted, A.S., Trauelsen, M., Rudenko, O., Hjorth, S.A., Schwartz, T.W., 2017. GPCR-mediated signaling of metabolites. Cell Metabolism 25:777-796.

[286] Ekberg, J.H., Hauge, M., Kristensen, L.V., Madsen, A.N., Engelstoft, M.S., Husted, A.S., et al., 2016. GPR119, a major enteroendocrine sensor of dietary triglyceride metabolites coacting in synergy with FFA1 (GPR40). Endocrinology 157:4561-4569.

[287] Kuhre, R.E., Wewer Albrechtsen, N.J., Deacon, C.F., Balk-Moller, E., Rehfeld, J.F., Reimann, F., et al., 2016. Peptide production and secretion in GLUTag, NCI-H716, and STC-1 cells: a comparison to native L-cells. Journal of Molecular Endocrinology 56:201-211.

[288] Roberge, J.N., Brubaker, P.L., 1991. Secretion of proglucagon-derived peptides in response to intestinal luminal nutrients. Endocrinology 128: $3169-3174$.

[289] Roberge, J.N., Brubaker, P.L., 1993. Regulation of intestinal proglucagonderived peptide secretion by glucose-dependent insulinotropic peptide in a novel enteroendocrine loop. Endocrinology 133:233-240.

[290] Thomsen, C., Rasmussen, O., Lousen, T., Holst, J.J., Fenselau, S. Schrezenmeir, J., et al., 1999. Differential effects of saturated and monounsaturated fatty acids on postprandial lipemia and incretin responses in healthy subjects. American Journal of Clinical Nutrition 69:1135-1143.

[291] Thomsen, C., Storm, H., Holst, J.J., Hermansen, K., 2003. Differential effects of saturated and monounsaturated fats on postprandial lipemia and glucagon-like peptide 1 responses in patients with type 2 diabetes. American Journal of Clinical Nutrition 77:605-611.

[292] Sidhu, S.S., Thompson, D.G., Warhurst, G., Case, R.M., Benson, R.S., 2000. Fatty acid-induced cholecystokinin secretion and changes in intracellular $\mathrm{Ca}^{2+}$ in two enteroendocrine cell lines, STC-1 and GLUTag. Journal of Physiology 528(Pt 1):165-176.

[293] Engelstoft, M.S., Schwartz, T.W., 2016. Opposite regulation of ghrelin and glucagon-like peptide-1 by metabolite G-protein-coupled receptors. Trends in Endocrinology and Metabolism 27:665-675.

[294] Edfalk, S., Steneberg, P., Edlund, H., 2008. Gpr40 is expressed in enteroendocrine cells and mediates free fatty acid stimulation of incretin secretion. Diabetes 57:2280-2287.

[295] Hauge, M., Vestmar, M.A., Husted, A.S., Ekberg, J.P., Wright, M.J., Di Salvo, J., et al., 2015. GPR40 (FFAR1) - combined Gs and Gq signaling 
in vitro is associated with robust incretin secretagogue action ex vivo and in vivo. Molecular Metabolism 4:3-14.

[296] Gorski, J.N., Pachanski, M.J., Mane, J., Plummer, C.W., Souza, S., ThomasFowlkes, B.S., et al., 2017. GPR40 reduces food intake and body weight through GLP-1. American Journal of Physiology Endocrinology and Metabolism 313:E37-E47.

[297] Xiong, Y., Swaminath, G., Cao, Q., Yang, L., Guo, Q., Salomonis, H., et al., 2013. Activation of FFA1 mediates GLP-1 secretion in mice. Evidence for allosterism at FFA1. Molecular and Cellular Endocrinology 369:119-129.

[298] Hansen, H.S., Rosenkilde, M.M., Holst, J.J., Schwartz, T.W., 2012. GPR119 as a fat sensor. Trends in Pharmacological Sciences 33:374-381.

[299] Mandoe, M.J., Hansen, K.B., Hartmann, B., Rehfeld, J.F., Holst, J.J., Hansen, H.S., 2015. The 2-monoacylglycerol moiety of dietary fat appears to be responsible for the fat-induced release of GLP-1 in humans. American Journal of Clinical Nutrition 102:548-555.

[300] Hauge, M., Ekberg, J.P., Engelstoft, M.S., Timshel, P., Madsen, A.N., Schwartz, T.W., 2017. Gq and Gs signaling acting in synergy to control GLP-1 secretion. Molecular and Cellular Endocrinology 449:64-73.

[301] Clara, R., Langhans, W., Mansouri, A., 2016. Oleic acid stimulates glucagonlike peptide-1 release from enteroendocrine cells by modulating cell respiration and glycolysis. Metabolism 65:8-17.

[302] Diakogiannaki, E., Pais, R., Tolhurst, G., Parker, H.E., Horscroft, J., Rauscher, B., et al., 2013. Oligopeptides stimulate glucagon-like peptide-1 secretion in mice through proton-coupled uptake and the calcium-sensing receptor. Diabetologia 56:2688-2696.

[303] Chen, Q., Reimer, R.A., 2009. Dairy protein and leucine alter GLP-1 release and mRNA of genes involved in intestinal lipid metabolism in vitro. Nutrition 25:340-349.

[304] Plaisancie, P., Dumoulin, V., Chayvialle, J.A., Cuber, J.C., 1995. Luminal glucagon-like peptide-1(7-36) amide-releasing factors in the isolated vascularly perfused rat colon. Journal of Endocrinology 145:521-526.

[305] Clemmensen, C., Smajilovic, S., Smith, E.P., Woods, S.C., BraunerOsborne, H., Seeley, R.J., et al., 2013. Oral L-arginine stimulates GLP-1 secretion to improve glucose tolerance in male mice. Endocrinology 154: 3978-3983.

[306] Kato, M., Nakanishi, T., Tani, T., Tsuda, T., 2017. Low-molecular fraction of wheat protein hydrolysate stimulates glucagon-like peptide-1 secretion in an enteroendocrine $L$ cell line and improves glucose tolerance in rats. Nutrition Research 37:37-45.

[307] Greenfield, J.R., Farooqi, I.S., Keogh, J.M., Henning, E., Habib, A.M., Blackwood, A., et al., 2009. Oral glutamine increases circulating glucagonlike peptide 1, glucagon, and insulin concentrations in lean, obese, and type 2 diabetic subjects. American Journal of Clinical Nutrition 89:106-113.

[308] Lejeune, M.P., Westerterp, K.R., Adam, T.C., Luscombe-Marsh, N.D., Westerterp-Plantenga, M.S., 2006. Ghrelin and glucagon-like peptide 1 concentrations, 24-h satiety, and energy and substrate metabolism during a high-protein diet and measured in a respiration chamber. American Journal of Clinical Nutrition 83:89-94.

[309] Floyd Jr., J.C., Fajans, S.S., Conn, J.W., Knopf, R.F., Rull, J., 1966. Stimulation of insulin secretion by amino acids. Journal of Clinical Investigation 45 : $1487-1502$.

[310] Reimer, R.A., 2006. Meat hydrolysate and essential amino acid-induced glucagon-like peptide-1 secretion, in the human $\mathrm{NCl}-\mathrm{H} 716$ enteroendocrine cell line, is regulated by extracellular signal-regulated kinase1/2 and p38 mitogen-activated protein kinases. Journal of Endocrinology 191:159-170.

[311] Calbet, J.A., Holst, J.J., 2004. Gastric emptying, gastric secretion and enterogastrone response after administration of milk proteins or their peptide hydrolysates in humans. European Journal of Nutrition 43:127-139.

[312] Mortensen, L.S., Hartvigsen, M.L., Brader, L.J., Astrup, A., Schrezenmeir, J., Holst, J.J., et al., 2009. Differential effects of protein quality on postprandial lipemia in response to a fat-rich meal in type 2 diabetes: comparison of whey, casein, gluten, and cod protein. American Journal of Clinical Nutrition 90:41-48.

[313] Nilsson, M., Stenberg, M., Frid, A.H., Holst, J.J., Bjorck, I.M., 2004. Glycemia and insulinemia in healthy subjects after lactose-equivalent meals of milk and other food proteins: the role of plasma amino acids and incretins. American Journal of Clinical Nutrition 80:1246-1253.

[314] Belza, A., Ritz, C., Sorensen, M.Q., Holst, J.J., Rehfeld, J.F., Astrup, A., 2013. Contribution of gastroenteropancreatic appetite hormones to protein-induced satiety. American Journal of Clinical Nutrition 97:980-989.

[315] Rudenko, 0., Shang, J., Munk, A., Ekberg, J.P., Petersen, N., Engelstoft, M.S., et al., 2019. The aromatic amino acid sensor GPR142 controls metabolism through balanced regulation of pancreatic and gut hormones. Molecular Metabolism 19:49-64.

[316] Theodorakis, M.J., Carlson, O., Michopoulos, S., Doyle, M.E., Juhaszova, M., Petraki, K., et al., 2006. Human duodenal enteroendocrine cells: source of both incretin peptides, GLP-1 and GIP. American Journal of Physiology Endocrinology and Metabolism 290:E550-E559.

[317] Jorsal, T., Rhee, N.A., Pedersen, J., Wahlgren, C.D., Mortensen, B., Jepsen, S.L., et al., 2018. Enteroendocrine $\mathrm{K}$ and L cells in healthy and type 2 diabetic individuals. Diabetologia 61:284-294.

[318] Svendsen, B., Pedersen, J., Albrechtsen, N.J., Hartmann, B., Torang, S., Rehfeld, J.F., et al., 2015. An analysis of cosecretion and coexpression of gut hormones from male rat proximal and distal small intestine. Endocrinology 156:847-857.

[319] Anini, Y., Hansotia, T., Brubaker, P.L., 2002. Muscarinic receptors control postprandial release of glucagon-like peptide-1: in vivo and in vitro studies in rats. Endocrinology 143:2420-2426.

[320] Lim, G.E., Brubaker, P.L., 2006. Glucagon-like peptide 1 secretion by the Lcell - the view from within. Diabetes 55:S70-S77.

[321] Lim, G.E., Huang, G.J., Flora, N., LeRoith, D., Rhodes, C.J., Brubaker, P.L., 2009. Insulin regulates glucagon-like peptide-1 secretion from the enteroendocrine L cell. Endocrinology 150:580-591.

[322] Gagnon, J., Baggio, L.L., Drucker, D.J., Brubaker, P.L., 2015. Ghrelin is a novel regulator of GLP-1 secretion. Diabetes 64:1513-1521.

[323] Brubaker, P.L., 1991. Regulation of intestinal proglucagon-derived peptide secretion by intestinal regulatory peptides. Endocrinology 128:3175-3182.

[324] Brubaker, P.L., Anini, Y., 2003. Direct and indirect mechanisms regulating secretion of glucagon-like peptide-1 and glucagon-like peptide-2. Canadian Journal of Physiology and Pharmacology 81:1005-1012.

[325] Cleator, I.G., Gourlay, R.H., 1975. Release of immunoreactive gastric inhibitory polypeptide (IR-GIP) by oral ingestion of food substances. Americas Journal of Surgery 130:128-135.

[326] Burhol, P.G., Waldum, H.L., Jorde, R., Lygren, I., 1979. The effect of a test meal on plasma vasoactive intestinal polypeptide (VIP), gastric inhibitory polypeptide (GIP), and secretin in man. Scandinavian Journal of Gastroenterology 14:939-943.

[327] Rocca, A.S., Brubaker, P.L., 1999. Role of the vagus nerve in mediating proximal nutrient-induced glucagon-like peptide-1 secretion. Endocrinology 140:1687-1694.

[328] Nauck, M.A., Heimesaat, M.M., Orskov, C., Holst, J.J., Ebert, R., Creutzfeldt, W., 1993. Preserved incretin activity of glucagon-like peptide 1 [7-36 amide] but not of synthetic human gastric inhibitory polypeptide in patients with type-2 diabetes mellitus. Journal of Clinical Investigation 91: $301-307$.

[329] Mentis, N., Vardarli, I., Kothe, L.D., Holst, J.J., Deacon, C.F., Theodorakis, M., et al., 2011. GIP does not potentiate the antidiabetic effects of GLP-1 in hyperglycemic patients with type 2 diabetes. Diabetes 60:1270-1276.

[330] Fieseler, P., Bridenbaugh, S., Nustede, R., Martell, J., Orskov, C., Holst, J.J., et al., 1995. Physiological augmentation of amino acid-induced insulin secretion by GIP and GLP-I but not by CCK-8. American Journal of Physiology 268:E949-E955. 
[331] Schirra, J., Katschinski, M., Weidmann, C., Schafer, T., Wank, U., Arnold, R., et al., 1996. Gastric emptying and release of incretin hormones after glucose ingestion in humans. Journal of Clinical Investigation 97:92-103.

[332] Zhang, X., Young, R.L., Bound, M., Hu, S., Jones, K.L., Horowitz, M., et al., 2019. Comparative effects of proximal and distal small intestinal glucose exposure on glycemia, incretin hormone secretion, and the incretin effect in health and type 2 diabetes. Diabetes Care 42:520-528.

[333] Beglinger, S., Drewe, J., Schirra, J., Goke, B., D’Amato, M., Beglinger, C., 2010. Role of fat hydrolysis in regulating glucagon-like Peptide-1 secretion. The Journal of Cinical Endocrinology and Metabolism 95:879-886.

[334] Hansen, L., Holst, J.J., 2002. The effects of duodenal peptides on glucagonlike peptide-1 secretion from the ileum. A duodeno-ileal loop? Regulatory Peptides 110:39-45.

[335] Herrmann-Rinke, C., Voge, A., Hess, M., Goke, B., 1995. Regulation of glucagon-like peptide- 1 secretion from rat ileum by neurotransmitters and peptides. Journal of Endocrinology 147:25-31.

[336] Anini, Y., Brubaker, P.L., 2003. Muscarinic receptors control glucagon-like peptide 1 secretion by human endocrine L cells. Endocrinology 144:32443250.

[337] Hansen, L., Lampert, S., Mineo, H., Holst, J.J., 2004. Neural regulation of glucagon-like peptide- 1 secretion in pigs. American Journal of Physiology Endocrinology and Metabolism 287:E939-E947.

[338] Moghimzadeh, E., Ekman, R., Hakanson, R., Yanaihara, N., Sundler, F., 1983. Neuronal gastrin-releasing peptide in the mammalian gut and pancreas. Neuroscience 10:553-563.

[339] Roberge, J.N., Gronau, K.A., Brubaker, P.L., 1996. Gastrin-releasing peptide is a novel mediator of proximal nutrient-induced proglucagon-derived peptide secretion from the distal gut. Endocrinology 137:2383-2388.

[340] Hermansen, K., Ahren, B., 1990. Gastrin releasing peptide stimulates the secretion of insulin, but not that of glucagon or somatostatin, from the isolated perfused dog pancreas. Acta Physiologica Scandinavica 138:175-179.

[341] Lenz, H.J., 1988. CNS regulation of gastric and autonomic functions in dogs by gastrin-releasing peptide. American Journal of Physiology 255:G298G303.

[342] Yegen, B.C., Gurbuz, V., Coskun, T., Bozkurt, A., Kurtel, H., Alican, I., et al., 1996. Inhibitory effects of gastrin releasing peptide on gastric emptying in rats. Regulatory Peptides 61:175-180.

[343] Persson, K., Gingerich, R.L., Nayak, S., Wada, K., Wada, E., Ahren, B., 2000. Reduced GLP-1 and insulin responses and glucose intolerance after gastric glucose in GRP receptor-deleted mice. American Journal of Physiology Endocrinology and Metabolism 279:E956-E962.

[344] Steinert, R.E., Feinle-Bisset, C., Asarian, L., Horowitz, M., Beglinger, C., Geary, N., 2017. Ghrelin, CCK, GLP-1, and PYY(3-36): secretory controls and physiological roles in eating and glycemia in health, obesity, and after RYGB. Physiological Reviews 97:411-463.

[345] Han, Y.E., Kang, C.W., Oh, J.H., Park, S.H., Ku, C.R., Cho, Y.H., et al., 2018. Olfactory receptor OR51E1 mediates GLP-1 secretion in human and rodent enteroendocrine $L$ cells. Journal of Endocrine Society 2:1251-1258.

[346] Llewellyn-Smith, I.J., Reimann, F., Gribble, F.M., Trapp, S., 2011. Preproglucagon neurons project widely to autonomic control areas in the mouse brain. Neuroscience 180:111-121.

[347] Merchenthaler, I., Lane, M., Shughrue, P., 1999. Distribution of pre-proglucagon and glucagon-like peptide-1 receptor messenger RNAs in the rat central nervous system. Journal of Comparative Neurology 403:261-280.

[348] Trapp, S., Hisadome, K., 2011. Glucagon-like peptide 1 and the brain: central actions-central sources? Autonomic Neuroscience 161:14-19.

[349] Elias, C.F., Kelly, J.F., Lee, C.E., Ahima, R.S., Drucker, D.J., Saper, C.B., et al., 2000. Chemical characterization of leptin-activated neurons in the rat brain. Journal of Comparative Neurology 423:261-281.

[350] Vrang, N., Phifer, C.B., Corkern, M.M., Berthoud, H.R., 2003. Gastric distension induces C-Fos in medullary GLP-1/2-containing neurons. American
Journal of Physiology Regulatory Integrative and Comparative Physiology 285: R470-R478.

[351] Hisadome, K., Reimann, F., Gribble, F.M., Trapp, S., 2010. Leptin directly depolarizes preproglucagon neurons in the nucleus tractus solitarius: electrical properties of glucagon-like Peptide 1 neurons. Diabetes 59:18901898.

[352] Hisadome, K., Reimann, F., Gribble, F.M., Trapp, S., 2011. CCK stimulation of GLP-1 neurons involves alpha1-adrenoceptor-mediated increase in glutamatergic synaptic inputs. Diabetes 60:2701-2709.

[353] Garfield, A.S., Patterson, C., Skora, S., Gribble, F.M., Reimann, F. Evans, M.L., et al., 2012. Neurochemical characterization of body weightregulating leptin receptor neurons in the nucleus of the solitary tract. Endocrinology 153:4600-4607.

[354] Rinaman, L., 1999. Interoceptive stress activates glucagon-like peptide-1 neurons that project to the hypothalamus. American Journal of Physiology 277:R582-R590.

[355] Baptista, V., Browning, K.N., Travagli, R.A., 2007. Effects of cholecystokinin$8 \mathrm{~s}$ in the nucleus tractus solitarius of vagally deafferented rats. American Journal of Physiology Regulatory Integrative and Comparative Physiology 292: R1092-R1100.

[356] Louis, D.S., 1990. Evolving concerns relating to occupational disorders of the upper extremity. Clinical Orthopaedics and Related Research, 140-143.

[357] Gaykema, R.P., Newmyer, B.A., Ottolini, M., Raje, V., Warthen, D.M., Lambeth, P.S., et al., 2017. Activation of murine pre-proglucagon-producing neurons reduces food intake and body weight. Journal of Clinical Investigation 127:1031-1045.

[358] Harmar, A.J., 2001. Family-B G-protein-coupled receptors. Genome Biology 2. REVIEWS3013.

[359] Mayo, K.E., Miller, L.J., Bataille, D., Dalle, S., Goke, B., Thorens, B., et al., 2003. International Union of Pharmacology. XXXV. The glucagon receptor family. Pharmacological Reviews 55:167-194.

[360] Thorens, B., 1992. Expression cloning of the pancreatic beta cell receptor for the gluco-incretin hormone glucagon-like peptide 1. Proceedings of the National Academy of Sciences of the United States of America 89:86418645.

[361] Dillon, J.S., Tanizawa, Y., Wheeler, M.B., Leng, X.H., Ligon, B.B., Rabin, D.U., et al., 1993. Cloning and functional expression of the human glucagon-like peptide-1 (GLP-1) receptor. Endocrinology 133:1907-1910.

[362] Graziano, M.P., Hey, P.J., Borkowski, D., Chicchi, G.G., Strader, C.D., 1993. Cloning and functional expression of a human glucagon-like peptide-1 receptor. Biochemical and Biophysical Research Communications 196:141146.

[363] Thorens, B., Porret, A., Buhler, L., Deng, S.P., Morel, P., Widmann, C., 1993. Cloning and functional expression of the human islet GLP-1 receptor. Demonstration that exendin-4 is an agonist and exendin-(9-39) an antagonist of the receptor. Diabetes 42:1678-1682.

[364] van Eyll, B., Lankat-Buttgereit, B., Bode, H.P., Goke, R., Goke, B., 1994. Signal transduction of the GLP-1-receptor cloned from a human insulinoma. FEBS Letters 348:7-13.

[365] Svendsen, B., Larsen, O., Gabe, M.B.N., Christiansen, C.B., Rosenkilde, M.M., Drucker, D.J., et al., 2018. Insulin secretion depends on intra-islet glucagon signaling. Cell Reports 25:1127-1134 e1122.

[366] Raufman, J.P., Singh, L., Singh, G., Eng, J., 1992. Truncated glucagon-like peptide-1 interacts with exendin receptors on dispersed acini from Guinea pig pancreas. Identification of a mammalian analogue of the reptilian peptide exendin-4. Journal of Biological Chemistry 267:21432-21437.

[367] Raufman, J.P., Singh, L., Eng, J., 1991. Exendin-3, a novel peptide from Heloderma horridum venom, interacts with vasoactive intestinal peptide receptors and a newly described receptor on dispersed acini from Guinea pig pancreas. Description of exendin-3(9-39) amide, a specific exendin receptor antagonist. Journal of Biological Chemistry 266:2897-2902. 
[368] Goke, R., Conlon, J.M., 1988. Receptors for glucagon-like peptide-1(7-36) amide on rat insulinoma-derived cells. Journal of Endocrinology 116:357362.

[369] Orskov, C., Nielsen, J.H., 1988. Truncated glucagon-like peptide-1 (proglucagon 78-107 amide), an intestinal insulin-releasing peptide, has specific receptors on rat insulinoma cells (RIN 5AH). FEBS Letters 229:175-178.

[370] Fehmann, H.C., Habener, J.F., 1991. Functional receptors for the insulinotropic hormone glucagon-like peptide-l(7-37) on a somatostatin secreting cell line. FEBS Letters 279:335-340.

[371] Fehmann, H.C., Habener, J.F., 1991. Homologous desensitization of the insulinotropic glucagon-like peptide-I (7-37) receptor on insulinoma (HIT-T15) cells. Endocrinology 128:2880-2888.

[372] Fehmann, H.C., Habener, J.F., 1992. Insulinotropic hormone glucagon-like peptide-I(7-37) stimulation of proinsulin gene expression and proinsulin biosynthesis in insulinoma beta TC-1 cells. Endocrinology 130:159-166.

[373] Fehmann, H.C., Habener, J.F., 1992. Galanin inhibits proinsulin gene expression stimulated by the insulinotropic hormone glucagon-like peptideI(7-37) in mouse insulinoma beta TC-1 cells. Endocrinology 130:28902896.

[374] Gallwitz, B., Witt, M., Folsch, U.R., Creutzfeldt, W., Schmidt, W.E., 1993. Binding specificity and signal transduction of receptors for glucagon-like peptide-1(7-36)amide and gastric inhibitory polypeptide on RINm5F insulinoma cells. Journal of Molecular Endocrinology 10:259-268.

[375] Gros, L., Demirpence, E., Jarrousse, C., Kervran, A., Bataille, D., 1992. Characterization of binding sites for oxyntomodulin on a somatostatinsecreting cell line (RIN T3). Endocrinology 130:1263-1270.

[376] Matsumura, T., Itoh, H., Watanabe, N., Oda, Y., Tanaka, M., Namba, M., et al., 1992. Glucagonlike peptide-1(7-36)amide suppresses glucagon secretion and decreases cyclic AMP concentration in cultured In-R1-G9 cells. Biochemical and Biophysical Research Communications 186:503-508.

[377] Moens, K., Heimberg, H., Flamez, D., Huypens, P., Quartier, E., Ling, Z., et al., 1996. Expression and functional activity of glucagon, glucagon-like peptide I, and glucose-dependent insulinotropic peptide receptors in rat pancreatic islet cells. Diabetes 45:257-261.

[378] Fehmann, H.C., Hering, B.J., Wolf, M.J., Brandhorst, H., Brandhorst, D., Bretzel, R.G., et al., 1995. The effects of glucagon-like peptide-I (GLP-I) on hormone secretion from isolated human pancreatic islets. Pancreas 11:196200.

[379] Lankat-Buttgereit, B., Goke, R., Fehmann, H.C., Richter, G., Goke, B., 1994. Molecular cloning of a cDNA encoding for the GLP-1 receptor expressed in rat lung. Experimental and Clinical Endocrinology 102:341-347.

[380] Bullock, B.P., Heller, R.S., Habener, J.F., 1996. Tissue distribution of messenger ribonucleic acid encoding the rat glucagon-like peptide-1 receptor. Endocrinology 137:2968-2978.

[381] Wei, Y., Mojsov, S., 1995. Tissue-specific expression of the human receptor for glucagon-like peptide-l: brain, heart and pancreatic forms have the same deduced amino acid sequences. FEBS Letters 358:219-224.

[382] Chen, J., Zhao, H., Ma, X., Zhang, Y., Lu, S., Wang, Y., et al., 2017. GLP-1/ GLP-1R signaling in regulation of adipocyte differentiation and lipogenesis. Cellular Physiology and Biochemistry 42:1165-1176.

[383] Egan, J.M., Montrose-Rafizadeh, C., Wang, Y., Bernier, M., Roth, J., 1994. Glucagon-like peptide-1(7-36) amide (GLP-1) enhances insulin-stimulated glucose metabolism in 3T3-L1 adipocytes: one of several potential extrapancreatic sites of GLP-1 action. Endocrinology 135:2070-2075.

[384] Challa, T.D., Beaton, N., Arnold, M., Rudofsky, G., Langhans, W., Wolfrum, C., 2012. Regulation of adipocyte formation by GLP-1/GLP-1R signaling. Journal of Biological Chemistry 287:6421-6430.

[385] Sanz, C., Vazquez, P., Blazquez, C., Barrio, P.A., Alvarez Mdel, M., Blazquez, E., 2010. Signaling and biological effects of glucagon-like peptide 1 on the differentiation of mesenchymal stem cells from human bone marrow. American Journal of Physiology. Endocrinology and Metabolism 298: E634-E643.

[386] lacobellis, G., Camarena, V., Sant, D.W., Wang, G., 2017. Human epicardial fat expresses glucagon-like peptide 1 and 2 receptors genes. Hormone and Metabolic Research 49:625-630.

[387] Vendrell, J., El Bekay, R., Peral, B., Garcia-Fuentes, E., Megia, A., MaciasGonzalez, M., et al., 2011. Study of the potential association of adipose tissue GLP-1 receptor with obesity and insulin resistance. Endocrinology 152: 4072-4079.

[388] Goke, R., Larsen, P.J., Mikkelsen, J.D., Sheikh, S.P., 1995. Distribution of GLP-1 binding sites in the rat brain: evidence that exendin- 4 is a ligand of brain GLP-1 binding sites. European Journal of Neuroscience 7:2294-2300.

[389] Heppner, K.M., Kirigiti, M., Secher, A., Paulsen, S.J., Buckingham, R., Pyke, C., et al., 2015. Expression and distribution of glucagon-like peptide-1 receptor mRNA, protein and binding in the male nonhuman primate (Macaca mulatta) brain. Endocrinology 156:255-267.

[390] Alvarez, E., Martinez, M.D., Roncero, I., Chowen, J.A., Garcia-Cuartero, B., Gispert, J.D., et al., 2005. The expression of GLP-1 receptor mRNA and protein allows the effect of GLP-1 on glucose metabolism in the human hypothalamus and brainstem. Journal of Neurochemistry 92:798-806.

[391] Panjwani, N., Mulvihill, E.E., Longuet, C., Yusta, B., Campbell, J.E., Brown, T.J., et al., 2013. GLP-1 receptor activation indirectly reduces hepatic lipid accumulation but does not attenuate development of atherosclerosis in diabetic male ApoE(-/-) mice. Endocrinology 154:127-139.

[392] Pyke, C., Heller, R.S., Kirk, R.K., Orskov, C., Reedtz-Runge, S., Kaastrup, P., et al., 2014. GLP-1 receptor localization in monkey and human tissue: novel distribution revealed with extensively validated monoclonal antibody. Endocrinology 155:1280-1290.

[393] Jensen, C.B., Pyke, C., Rasch, M.G., Dahl, A.B., Knudsen, L.B., Secher, A., 2018. Characterization of the glucagonlike peptide-1 receptor in male mouse brain using a novel antibody and in situ hybridization. Endocrinology 159: $665-675$.

[394] Pyke, C., Knudsen, L.B., 2013. The glucagon-like peptide-1 receptor-or not? Endocrinology 154:4-8.

[395] Richards, P., Parker, H.E., Adriaenssens, A.E., Hodgson, J.M., Cork, S.C., Trapp, S., et al., 2014. Identification and characterization of GLP-1 receptorexpressing cells using a new transgenic mouse model. Diabetes 63:12241233.

[396] Cork, S.C., Richards, J.E., Holt, M.K., Gribble, F.M., Reimann, F., Trapp, S., 2015. Distribution and characterisation of Glucagon-like peptide-1 receptor expressing cells in the mouse brain. Molecular Metabolism 4:718-731.

[397] Muraro, M.J., Dharmadhikari, G., Grun, D., Groen, N., Dielen, T., Jansen, E., et al., 2016. A single-cell transcriptome atlas of the human pancreas. Cellular Systems 3:385-394 e383.

[398] Segerstolpe, A., Palasantza, A., Eliasson, P., Andersson, E.M., Andreasson, A.C., Sun, X., et al., 2016. Single-cell transcriptome profiling of human pancreatic islets in health and type 2 diabetes. Cell Metabolism 24: 593-607.

[399] Waser, B., Blank, A., Karamitopoulou, E., Perren, A., Reubi, J.C., 2015. Glucagon-like-peptide-1 receptor expression in normal and diseased human thyroid and pancreas. Modern Pathology 28:391-402.

[400] Ast, J., Arvaniti, A., Fine, N.H.F., Nasteska, D., Ashford, F.B., Stamataki, Z., et al., 2019. LUXendins reveal endogenous glucagon-like peptide-1 receptor distribution and dynamics. bioRxiv, 557132.

[401] Moon, M.J., Park, S., Kim, D.K., Cho, E.B., Hwang, J.l., Vaudry, H., et al., 2012. Structural and molecular conservation of glucagon-like Peptide-1 and its receptor confers selective ligand-receptor interaction. Frontiers in Endocrinology (Lausanne) 3:141.

[402] Alken, M., Rutz, C., Kochl, R., Donalies, U., Oueslati, M., Furkert, J., et al., 2005. The signal peptide of the rat corticotropin-releasing factor receptor 1 
promotes receptor expression but is not essential for establishing a functional receptor. Biochemical Journal 390:455-464.

[403] Couvineau, A., Rouyer-Fessard, C., Laburthe, M., 2004. Presence of a Nterminal signal peptide in class II G protein-coupled receptors: crucial role for expression of the human VPAC1 receptor. Regulatory Peptides 123:181185.

[404] Huang, Y., Wilkinson, G.F., Willars, G.B., 2010. Role of the signal peptide in the synthesis and processing of the glucagon-like peptide-1 receptor. British Journal of Pharmacology 159:237-251.

[405] Parthier, C., Kleinschmidt, M., Neumann, P., Rudolph, R., Manhart, S., Schlenzig, D., et al., 2007. Crystal structure of the incretin-bound extracelIular domain of a G protein-coupled receptor. Proceedings of the National Academy of Sciences of the United States of America 104:13942-13947.

[406] Parthier, C., Reedtz-Runge, S., Rudolph, R., Stubbs, M.T., 2009. Passing the baton in class B GPCRs: peptide hormone activation via helix induction? Trends in Biochemical Sciences 34:303-310.

[407] Runge, S., Thogersen, H., Madsen, K., Lau, J., Rudolph, R., 2008. Crystal structure of the ligand-bound glucagon-like peptide-1 receptor extracellular domain. Journal of Biological Chemistry 283:11340-11347.

[408] Tan, Y.V., Couvineau, A., Murail, S., Ceraudo, E., Neumann, J.M., Lacapere, J.J., et al., 2006. Peptide agonist docking in the N-terminal ectodomain of a class II G protein-coupled receptor, the VPAC1 receptor. Photoaffinity, NMR, and molecular modeling. Journal of Biological Chemistry 281:12792-12798.

[409] Underwood, C.R., Garibay, P., Knudsen, L.B., Hastrup, S., Peters, G.H., Rudolph, R., et al., 2010. Crystal structure of glucagon-like peptide-1 in complex with the extracellular domain of the glucagon-like peptide-1 receptor. Journal of Biological Chemistry 285:723-730.

[410] Adelhorst, K., Hedegaard, B.B., Knudsen, L.B., Kirk, O., 1994. Structureactivity studies of glucagon-like peptide-1. Journal of Biological Chemistry 269:6275-6278.

[411] Gallwitz, B., Witt, M., Paetzold, G., Morys-Wortmann, C., Zimmermann, B., Eckart, K., et al., 1994. Structure/activity characterization of glucagon-like peptide-1. European Journal of Biochemistry 225:1151-1156.

[412] Hinke, S.A., Manhart, S., Speck, M., Pederson, R.A., Demuth, H.U., McIntosh, C.H., 2004. In depth analysis of the N-terminal bioactive domain of gastric inhibitory polypeptide. Life Sciences 75:1857-1870.

[413] Graziano, M.P., Hey, P.J., Strader, C.D., 1996. The amino terminal domain of the glucagon-like peptide- 1 receptor is a critical determinant of subtype specificity. Receptors and Channels 4:9-17.

[414] Runge, S., Wulff, B.S., Madsen, K., Brauner-Osborne, H., Knudsen, L.B., 2003. Different domains of the glucagon and glucagon-like peptide-1 receptors provide the critical determinants of ligand selectivity. British Journal of Pharmacology 138:787-794.

[415] Van Eyll, B., Goke, B., Wilmen, A., Goke, R., 1996. Exchange of W39 by A within the N-terminal extracellular domain of the GLP-1 receptor results in a loss of receptor function. Peptides 17:565-570.

[416] Wilmen, A., Goke, B., Goke, R., 1996. The isolated N-terminal extracellular domain of the glucagon-like peptide-1 (GLP)-1 receptor has intrinsic binding activity. FEBS Letters 398:43-47.

[417] During, M.J., Cao, L., Zuzga, D.S., Francis, J.S., Fitzsimons, H.L., Jiao, X., et al., 2003. Glucagon-like peptide-1 receptor is involved in learning and neuroprotection. Nature Medicine 9:1173-1179.

[418] Moon, M.J., Kim, H.Y., Kim, S.G., Park, J., Choi, D.S., Hwang, J.I., et al., 2010. Tyr1 and lle7 of glucose-dependent insulinotropic polypeptide (GIP) confer differential ligand selectivity toward GIP and glucagon-like peptide-1 receptors. Molecules and Cells 30:149-154.

[419] Moon, M.J., Kim, H.Y., Park, S., Kim, D.K., Cho, E.B., Park, C.R., et al., 2012. Evolutionarily conserved residues at glucagon-like peptide-1 (GLP-1) receptor core confer ligand-induced receptor activation. Journal of Biological Chemistry 287:3873-3884.
[420] Moon, M.J., Lee, Y.N., Park, S., Reyes-Alcaraz, A., Hwang, J.I., Millar, R.P. et al., 2015. Ligand binding pocket formed by evolutionarily conserved residues in the glucagon-like peptide-1 (GLP-1) receptor core domain. Journal of Biological Chemistry 290:5696-5706.

[421] Al-Sabah, S., Donnelly, D., 2003. A model for receptor-peptide binding at the glucagon-like peptide-1 (GLP-1) receptor through the analysis of truncated ligands and receptors. British Journal of Pharmacology 140:339-346.

[422] Lopez de Maturana, R., Willshaw, A., Kuntzsch, A., Rudolph, R., Donnelly, D. 2003. The isolated N-terminal domain of the glucagon-like peptide-1 (GLP-1) receptor binds exendin peptides with much higher affinity than GLP-1. Journal of Biological Chemistry 278:10195-10200.

[423] Song, G., Yang, D., Wang, Y., de Graaf, C., Zhou, Q., Jiang, S., et al., 2017. Human GLP-1 receptor transmembrane domain structure in complex with allosteric modulators. Nature 546:312-315.

[424] Zhang, Y., Sun, B., Feng, D., Hu, H., Chu, M., Qu, Q., et al., 2017. Cryo-EM structure of the activated GLP-1 receptor in complex with a $\mathrm{G}$ protein. Nature 546:248-253.

[425] Jazayeri, A., Rappas, M., Brown, A.J.H., Kean, J., Errey, J.C., Robertson, N.J., et al., 2017. Crystal structure of the GLP-1 receptor bound to a peptide agonist. Nature 546:254-258.

[426] Rankovic, Z., Brust, T.F., Bohn, L.M., 2016. Biased agonism: an emerging paradigm in GPCR drug discovery. Bioorganic and Medicinal Chemistry Letters 26:241-250.

[427] Koole, C., Wootten, D., Simms, J., Valant, C., Sridhar, R., Woodman, O.L., et al., 2010. Allosteric ligands of the glucagon-like peptide 1 receptor (GLP$1 \mathrm{R})$ differentially modulate endogenous and exogenous peptide responses in a pathway-selective manner: implications for drug screening. Molecular Pharmacology 78:456-465.

[428] Montrose-Rafizadeh, C., Avdonin, P., Garant, M.J., Rodgers, B.D., Kole, S., Yang, H., et al., 1999. Pancreatic glucagon-like peptide-1 receptor couples to multiple G proteins and activates mitogen-activated protein kinase pathways in Chinese hamster ovary cells. Endocrinology 140:1132-1140.

[429] Sonoda, N., Imamura, T., Yoshizaki, T., Babendure, J.L., Lu, J.C., Olefsky, J.M., 2008. Beta-Arrestin-1 mediates glucagon-like peptide-1 signaling to insulin secretion in cultured pancreatic beta cells. Proceedings of the National Academy of Sciences of the United States of America 105: 6614-6619.

[430] Wheeler, M.B., Lu, M., Dillon, J.S., Leng, X.H., Chen, C., Boyd 3rd., A.E., 1993. Functional expression of the rat glucagon-like peptide-I receptor, evidence for coupling to both adenylyl cyclase and phospholipase-C. Endocrinology 133:57-62.

[431] Wootten, D., Reynolds, C.A., Smith, K.J., Mobarec, J.C., Koole, C., Savage, E.E., et al., 2016. The extracellular surface of the GLP-1 receptor is a molecular trigger for biased agonism. Cell 165:1632-1643.

[432] Wootten, D., Savage, E.E., Willard, F.S., Bueno, A.B., Sloop, K.W., Christopoulos, A., et al., 2013. Differential activation and modulation of the glucagon-like peptide-1 receptor by small molecule ligands. Molecular Pharmacology 83:822-834.

[433] Quoyer, J., Longuet, C., Broca, C., Linck, N., Costes, S., Varin, E., et al., 2010. GLP-1 mediates antiapoptotic effect by phosphorylating Bad through a beta-arrestin 1-mediated ERK1/2 activation in pancreatic beta-cells. Journal of Biological Chemistry 285:1989-2002.

[434] Zhang, H., Sturchler, E., Zhu, J., Nieto, A., Cistrone, P.A., Xie, J., et al., 2015. Autocrine selection of a GLP-1R G-protein biased agonist with potent antidiabetic effects. Nature Communications 6:8918.

[435] Liang, Y.L., Khoshouei, M., Glukhova, A., Furness, S.G.B., Zhao, P., Clydesdale, L., et al., 2018. Phase-plate cryo-EM structure of a biased agonist-bound human GLP-1 receptor-Gs complex. Nature 555:121-125.

[436] Ferguson, S.S., Barak, L.S., Zhang, J., Caron, M.G., 1996. G-protein-coupled receptor regulation: role of G-protein-coupled receptor kinases and arrestins. Canadian Journal of Physiology and Pharmacology 74:1095-1110. 
[437] Krupnick, J.G., Benovic, J.L., 1998. The role of receptor kinases and arrestins in G protein-coupled receptor regulation. Annual Review of Pharmacology and Toxicology 38:289-319.

[438] Pak, Y., Kouvelas, A., Scheideler, M.A., Rasmussen, J., O'Dowd, B.F., George, S.R., 1996. Agonist-induced functional desensitization of the muopioid receptor is mediated by loss of membrane receptors rather than uncoupling from G protein. Molecular Pharmacology 50:1214-1222.

[439] Yang, J., Williams, J.A., Yule, D.I., Logsdon, C.D., 1995. Mutation of carboxylterminal threonine residues in human $\mathrm{m} 3$ muscarinic acetylcholine receptor modulates the extent of sequestration and desensitization. Molecular Pharmacology 48:477-485.

[440] Chuang, D.M., Kinnier, W.J., Farber, L., Costa, E., 1980. A biochemical study of receptor internalization during beta-adrenergic receptor desensitization in frog erythrocytes. Molecular Pharmacology 18:348-355.

[441] Hertel, C., Coulter, S.J., Perkins, J.P., 1985. A comparison of catecholamineinduced internalization of beta-adrenergic receptors and receptor-mediated endocytosis of epidermal growth factor in human astrocytoma cells. Inhibition by phenylarsine oxide. Journal of Biological Chemistry 260:1254712553.

[442] Homburger, V., Lucas, M., Cantau, B., Barabe, J., Penit, J., Bockaert, J., 1980. Further evidence that desensitization of beta-adrenergic-sensitive adenylate cyclase proceeds in two steps. Modification of the coupling and loss of beta-adrenergic receptors. Journal of Biological Chemistry 255: 10436-10444.

[443] Pippig, S., Andexinger, S., Lohse, M.J., 1995. Sequestration and recycling of beta 2-adrenergic receptors permit receptor resensitization. Molecular Pharmacology 47:666-676.

[444] Thomas, W.G., Thekkumkara, T.J., Motel, T.J., Baker, K.M., 1995. Stable expression of a truncated AT1A receptor in $\mathrm{CHO}-\mathrm{K} 1$ cells. The carboxylterminal region directs agonist-induced internalization but not receptor signaling or desensitization. Journal of Biological Chemistry 270:207-213.

[445] Ng, G.Y., Trogadis, J., Stevens, J., Bouvier, M., O'Dowd, B.F., George, S.R., 1995. Agonist-induced desensitization of dopamine D1 receptor-stimulated adenylyl cyclase activity is temporally and biochemically separated from D1 receptor internalization. Proceedings of the National Academy of Sciences of the United States of America 92:10157-10161.

[446] Pals-Rylaarsdam, R., Xu, Y., Witt-Enderby, P., Benovic, J.L., Hosey, M.M., 1995. Desensitization and internalization of the $\mathrm{m} 2$ muscarinic acetylcholine receptor are directed by independent mechanisms. Journal of Biological Chemistry 270:29004-29011.

[447] Garland, A.M., Grady, E.F., Lovett, M., Vigna, S.R., Frucht, M.M., Krause, J.E., et al., 1996. Mechanisms of desensitization and resensitization of $G$ proteincoupled neurokinin1 and neurokinin2 receptors. Molecular Pharmacology 49: 438-446.

[448] Fukushima, Y., Asano, T., Takata, K., Funaki, M., Ogihara, T., Anai, M., et al., 1997. Role of the C terminus in histamine $\mathrm{H} 2$ receptor signaling, desensitization, and agonist-induced internalization. Journal of Biological Chemistry 272:19464-19470.

[449] Holtmann, M.H., Roettger, B.F., Pinon, D.I., Miller, L.J., 1996. Role of receptor phosphorylation in desensitization and internalization of the secretin receptor. Journal of Biological Chemistry 271:23566-23571.

[450] Kuna, R.S., Girada, S.B., Asalla, S., Vallentyne, J., Maddika, S., Patterson, J.T., et al., 2013. Glucagon-like peptide-1 receptor-mediated endosomal cAMP generation promotes glucose-stimulated insulin secretion in pancreatic beta-cells. American Journal of Physiology Endocrinology and Metabolism 305:E161-E170.

[451] Thompson, A., Kanamarlapudi, V., 2015. Agonist-induced internalisation of the glucagon-like peptide-1 receptor is mediated by the Galphaq pathway. Biochemical Pharmacology 93:72-84.

[452] Girada, S.B., Kuna, R.S., Bele, S., Zhu, Z., Chakravarthi, N.R., DiMarchi, R.D., et al., 2017. Galphas regulates Glucagon-Like Peptide 1 Receptor-mediated cyclic AMP generation at Rab5 endosomal compartment. Molecular Metabolism 6:1173-1185.

[453] Widmann, C., Dolci, W., Thorens, B., 1995. Agonist-induced internalization and recycling of the glucagon-like peptide-1 receptor in transfected fibroblasts and in insulinomas. Biochemical Journal 310(Pt 1):203-214.

[454] Goke, R., Richter, G., Goke, B., Trautmann, M., Arnold, R., 1989. Internalization of glucagon-like peptide-1(7-36)amide in rat insulinoma cells. Research in Experimental Medicine(Berlin) 189:257-264.

[455] Jones, B., Buenaventura, T., Kanda, N., Chabosseau, P., Owen, B.M., Scott, R., et al., 2018. Targeting GLP-1 receptor trafficking to improve agonist efficacy. Nature Communications 9:1602.

[456] Vilardaga, J.P., Romero, G., Friedman, P.A., Gardella, T.J., 2011. Molecular basis of parathyroid hormone receptor signaling and trafficking: a family $B$ GPCR paradigm. Cellular and Molecular Life Sciences 68:1-13.

[457] Calebiro, D., Nikolaev, V.0., Gagliani, M.C., de Filippis, T., Dees, C., Tacchetti, C., et al., 2009. Persistent CAMP-signals triggered by internalized G-protein-coupled receptors. PLoS Biology 7:e1000172.

[458] Mullershausen, F., Zecri, F., Cetin, C., Billich, A., Guerini, D., Seuwen, K., 2009. Persistent signaling induced by FTY720-phosphate is mediated by internalized S1P1 receptors. Nature Chemical Biology 5:428-434.

[459] Hothersall, J.D., Brown, A.J., Dale, I., Rawlins, P., 2016. Can residence time offer a useful strategy to target agonist drugs for sustained GPCR responses? Drug Discovery Today 21:90-96.

[460] Scrocchi, L.A., Brown, T.J., MaClusky, N., Brubaker, P.L., Auerbach, A.B., Joyner, A.L., et al., 1996. Glucose intolerance but normal satiety in mice with a null mutation in the glucagon-like peptide 1 receptor gene. Nature Medicine 2:1254-1258.

[461] Scrocchi, L.A., Marshall, B.A., Cook, S.M., Brubaker, P.L., Drucker, D.J., 1998. Identification of glucagon-like peptide 1 (GLP-1) actions essential for glucose homeostasis in mice with disruption of GLP-1 receptor signaling. Diabetes 47:632-639.

[462] Scrocchi, L.A., Drucker, D.J., 1998. Effects of aging and a high fat diet on body weight and glucose tolerance in glucagon-like peptide-1 receptor -/mice. Endocrinology 139:3127-3132.

[463] Goke, R., Fehmann, H.C., Linn, T., Schmidt, H., Krause, M., Eng, J., et al., 1993. Exendin-4 is a high potency agonist and truncated exendin-(9-39)amide an antagonist at the glucagon-like peptide 1-(7-36)-amide receptor of insulin-secreting beta-cells. Journal of Biological Chemistry 268:1965019655.

[464] Wang, Y., Egan, J.M., Raygada, M., Nadiv, 0., Roth, J., MontroseRafizadeh, C., 1995. Glucagon-like peptide-1 affects gene transcription and messenger ribonucleic acid stability of components of the insulin secretory system in RIN 1046-38 cells. Endocrinology 136:4910-4917.

[465] Finan, B., Ma, T., Ottaway, N., Muller, T.D., Habegger, K.M., Heppner, K.M., et al., 2013. Unimolecular dual incretins maximize metabolic benefits in rodents, monkeys, and humans. Science Translational Medicine 5, 209ra151.

[466] Finan, B., Yang, B., Ottaway, N., Smiley, D.L., Ma, T., Clemmensen, C., et al., 2015. A rationally designed monomeric peptide triagonist corrects obesity and diabetes in rodents. Nature Medicine 21:27-36.

[467] Finan, B., Yang, B., Ottaway, N., Stemmer, K., Muller, T.D., Yi, C.X., et al., 2012. Targeted estrogen delivery reverses the metabolic syndrome. Nature Medicine 18:1847-1856.

[468] Ahren, B., Hughes, T.E., 2005. Inhibition of dipeptidyl peptidase-4 augments insulin secretion in response to exogenously administered glucagon-like peptide-1, glucose-dependent insulinotropic polypeptide, pituitary adenylate cyclase-activating polypeptide, and gastrin-releasing peptide in mice. Endocrinology 146:2055-2059.

[469] Fernandez, J., Valdeolmillos, M., 1999. Glucose-dependent stimulatory effect of glucagon-like peptide 1(7-36) amide on the electrical activity of pancreatic beta-cells recorded in vivo. Diabetes 48:754-757. 
[470] Simonsson, E., Ahren, B., 1998. Potentiated beta-cell response to nonglucose stimuli in insulin-resistant C57BL/6J mice. European Journal of Pharmacology 350:243-250.

[471] Ahren, B., Lindskog, S., van Dijk, G., Scheurink, A.J., Steffens, A.B., 1995. Effects of GLP-1 and 2,5-anhydro-D-mannitol on insulin secretion and plasma glucose in mice. Endocrine Research 21:583-594.

[472] Fehmann, H.C., Goke, B., Goke, R., Trautmann, M.E., Arnold, R., 1989. Synergistic stimulatory effect of glucagon-like peptide-1 (7-36) amide and glucose-dependent insulin-releasing polypeptide on the endocrine rat pancreas. FEBS Letters 252:109-112.

[473] Kawai, K., Suzuki, S., Ohashi, S., Mukai, H., Ohmori, H., Murayama, Y., et al., 1989. Comparison of the effects of glucagon-like peptide-1-(1-37) and -(737) and glucagon on islet hormone release from isolated perfused canine and rat pancreases. Endocrinology 124:1768-1773.

[474] Komatsu, R., Matsuyama, T., Namba, M., Watanabe, N., Itoh, H., Kono, N., et al., 1989. Glucagonostatic and insulinotropic action of glucagonlike peptide I-(7-36)-amide. Diabetes 38:902-905.

[475] Orskov, C., Holst, J.J., Nielsen, O.V., 1988. Effect of truncated glucagon-like peptide-1 [proglucagon-(78-107) amide] on endocrine secretion from pig pancreas, antrum, and nonantral stomach. Endocrinology 123:2009-2013.

[476] Gromada, J., Ding, W.G., Barg, S., Renstrom, E., Rorsman, P., 1997. Multisite regulation of insulin secretion by CAMP-increasing agonists: evidence that glucagon-like peptide 1 and glucagon act via distinct receptors. Pflügers Archiv 434:515-524.

[477] Clark, S.A., Burnham, B.L., Chick, W.L., 1990. Modulation of glucose-induced insulin secretion from a rat clonal beta-cell line. Endocrinology 127:27792788.

[478] Gromada, J., Dissing, S., Bokvist, K., Renstrom, E., Frokjaer-Jensen, J., Wulff, B.S., et al., 1995. Glucagon-like peptide I increases cytoplasmic calcium in insulin-secreting beta TC3-cells by enhancement of intracellular calcium mobilization. Diabetes 44:767-774.

[479] Gromada, J., Dissing, S., Rorsman, P., 1996. Desensitization of glucagon-like peptide 1 receptors in insulin-secreting beta TC3 cells: role of PKAindependent mechanisms. British Journal of Pharmacology 118:769-775.

[480] Weir, G.C., Mojsov, S., Hendrick, G.K., Habener, J.F., 1989. Glucagonlike peptide I (7-37) actions on endocrine pancreas. Diabetes 38:338-342.

[481] Hansotia, T., Baggio, L.L., Delmeire, D., Hinke, S.A., Yamada, Y., Tsukiyama, K., et al., 2004. Double incretin receptor knockout (DIRKO) mice reveal an essential role for the enteroinsular axis in transducing the glucoregulatory actions of DPP-IV inhibitors. Diabetes 53:1326-1335.

[482] Hansotia, T., Drucker, D.J., 2005. GIP and GLP-1 as incretin hormones: lessons from single and double incretin receptor knockout mice. Regulatory Peptides 128:125-134.

[483] Preitner, F., Ibberson, M., Franklin, I., Binnert, C., Pende, M., Gjinovci, A., et al., 2004. Gluco-incretins control insulin secretion at multiple levels as revealed in mice lacking GLP-1 and GIP receptors. Journal of Clinical Investigation 113:635-645.

[484] Montrose-Rafizadeh, C., Wang, Y., Janczewski, A.M., Henderson, T.E., Egan, J.M., 1997. Overexpression of glucagon-like peptide-1 receptor in an insulin-secreting cell line enhances glucose responsiveness. Molecular and Cellular Endocrinology 130:109-117.

[485] Harndahl, L., Jing, X.J., Ivarsson, R., Degerman, E., Ahren, B., Manganiello, V.C., et al., 2002. Important role of phosphodiesterase 3B for the stimulatory action of CAMP on pancreatic beta-cell exocytosis and release of insulin. Journal of Biological Chemistry 277:37446-37455.

[486] Zhao, A.Z., Zhao, H., Teague, J., Fujimoto, W., Beavo, J.A., 1997. Attenuation of insulin secretion by insulin-like growth factor 1 is mediated through activation of phosphodiesterase 3B. Proceedings of the National Academy of Sciences of the United States of America 94:3223-3228.

[487] Harndahl, L., Wierup, N., Enerback, S., Mulder, H., Manganiello, V.C., Sundler, F., et al., 2004. Beta-cell-targeted overexpression of phosphodiesterase 3B in mice causes impaired insulin secretion, glucose intolerance, and deranged islet morphology. Journal of Biological Chemistry 279:15214-15222.

[488] Doyle, M.E., Egan, J.M., 2007. Mechanisms of action of glucagon-like peptide 1 in the pancreas. Pharmacology and Therapeutics 113:546-593.

[489] Holz, G.G., 2004. Epac: a new cAMP-binding protein in support of glucagonlike peptide- 1 receptor-mediated signal transduction in the pancreatic betacell. Diabetes 53:5-13.

[490] Wang, X., Zhou, J., Doyle, M.E., Egan, J.M., 2001. Glucagon-like peptide-1 causes pancreatic duodenal homeobox-1 protein translocation from the cytoplasm to the nucleus of pancreatic beta-cells by a cyclic adenosine monophosphate/protein kinase A-dependent mechanism. Endocrinology 142: $1820-1827$.

[491] Bunemann, M., Gerhardstein, B.L., Gao, T., Hosey, M.M., 1999. Functional regulation of L-type calcium channels via protein kinase A-mediated phosphorylation of the beta(2) subunit. Journal of Biological Chemistry 274: 33851-33854.

[492] MacDonald, P.E., Wang, X., Xia, F., El-kholy, W., Targonsky, E.D. Tsushima, R.G., et al., 2003. Antagonism of rat beta-cell voltage-dependent $\mathrm{K}+$ currents by exendin 4 requires dual activation of the CAMP/protein kinase A and phosphatidylinositol 3-kinase signaling pathways. Journal of Biological Chemistry 278:52446-52453.

[493] Kashima, Y., Miki, T., Shibasaki, T., Ozaki, N., Miyazaki, M., Yano, H., et al., 2001. Critical role of CAMP-GEFII-Rim2 complex in incretin-potentiated insulin secretion. Journal of Biological Chemistry 276:46046-46053.

[494] de Rooij, J., Zwartkruis, F.J., Verheijen, M.H., Cool, R.H., Nijman, S.M., Wittinghofer, A., et al., 1998. Epac is a Rap1 guanine-nucleotide-exchange factor directly activated by cyclic AMP. Nature 396:474-477.

[495] Kawasaki, H., Springett, G.M., Mochizuki, N., Toki, S., Nakaya, M., Matsuda, M., et al., 1998. A family of CAMP-binding proteins that directly activate Rap1. Science 282:2275-2279.

[496] Cheng, X., Ji, Z., Tsalkova, T., Mei, F., 2008. Epac and PKA: a tale of two intracellular cAMP receptors. Acta Biochimica et Biophysica Sinica (Shanghai) 40:651-662.

[497] Leech, C.A., Holz, G.G., Chepurny, O., Habener, J.F., 2000. Expression of CAMP-regulated guanine nucleotide exchange factors in pancreatic betacells. Biochemical and Biophysical Research Communications 278:44-47.

[498] Holz, G.G., Leech, C.A., Heller, R.S., Castonguay, M., Habener, J.F., 1999. CAMP-dependent mobilization of intracellular $\mathrm{Ca}^{2+}$ stores by activation of ryanodine receptors in pancreatic beta-cells. $\mathrm{A} \mathrm{Ca}^{2+}$ signaling system stimulated by the insulinotropic hormone glucagon-like peptide-1-(7-37). Journal of Biological Chemistry 274:14147-14156.

[499] Kang, G., Chepurny, 0.G., Holz, G.G., 2001. cAMP-regulated guanine nucleotide exchange factor II (Epac2) mediates $\mathrm{Ca}^{2+}$-induced $\mathrm{Ca}^{2+}$ release in INS-1 pancreatic beta-cells. Journal of Physiology 536:375-385.

[500] Tsuboi, T., da Silva Xavier, G., Holz, G.G., Jouaville, L.S., Thomas, A.P., Rutter, G.A., 2003. Glucagon-like peptide-1 mobilizes intracellular $\mathrm{Ca}^{2+}$ and stimulates mitochondrial ATP synthesis in pancreatic MIN6 beta-cells. Biochemical Journal 369:287-299.

[501] Irie, S., Matsumura, Y., Zdravkovic, M., Jacobsen, L.V., Kageyama, S., 2008. Tolerability, pharmacokinetics and pharmacodynamics of the once-daily human GLP-1 analog liraglutide in Japanese healthy subjects: a randomized, double-blind, placebo-controlled dose-escalation study. International Journal of Clinical Pharmacology and Therapeutics 46:273-279.

[502] Seino, Y., Rasmussen, M.F., Zdravkovic, M., Kaku, K., 2008. Dose-dependent improvement in glycemia with once-daily liraglutide without hypoglycemia or weight gain: a double-blind, randomized, controlled trial in Japanese patients with type 2 diabetes. Diabetes Research and Clinical Practice 81:161-168.

[503] Vilsboll, T., Zdravkovic, M., Le-Thi, T., Krarup, T., Schmitz, 0. Courreges, J.P., et al., 2007. Liraglutide, a long-acting human glucagon-like peptide-1 analog, given as monotherapy significantly improves glycemic 
control and lowers body weight without risk of hypoglycemia in patients with type 2 diabetes. Diabetes Care 30:1608-1610.

[504] Ponzani, P., Scardapane, M., Nicolucci, A., Rossi, M.C., 2016. Effectiveness and safety of liraglutide after three years of treatment. Minerva Endocrinologica 41:35-42.

[505] Tanaka, K., Saisho, Y., Kawai, T., Tanaka, M., Meguro, S., Irie, J., et al., 2015. Efficacy and safety of liraglutide monotherapy compared with metformin in Japanese overweight/obese patients with type 2 diabetes. Endocrine Journal 62:399-409.

[506] Vilsboll, T., Krarup, T., Madsbad, S., Holst, J.J., 2001. No reactive hypoglycaemia in Type 2 diabetic patients after subcutaneous administration of GLP-1 and intravenous glucose. Diabetic Medicine 18:144-149.

[507] Nauck, M.A., Heimesaat, M.M., Behle, K., Holst, J.J., Nauck, M.S., Ritzel, R., et al., 2002. Effects of glucagon-like peptide 1 on counterregulatory hormone responses, cognitive functions, and insulin secretion during hyperinsulinemic, stepped hypoglycemic clamp experiments in healthy volunteers. The Journal of Cinical Endocrinology and Metabolism 87:1239-1246.

[508] Gromada, J., Brock, B., Schmitz, 0., Rorsman, P., 2004. Glucagon-like peptide-1: regulation of insulin secretion and therapeutic potential. Basic and Clinical Pharmacology and Toxicology 95:252-262.

[509] Renstrom, E., Eliasson, L., Rorsman, P., 1997. Protein kinase A-dependent and -independent stimulation of exocytosis by CAMP in mouse pancreatic Bcells. Journal of Physiology 502(Pt 1):105-118.

[510] Gromada, J., Bokvist, K., Ding, W.G., Holst, J.J., Nielsen, J.H., Rorsman, P., 1998. Glucagon-like peptide 1 (7-36) amide stimulates exocytosis in human pancreatic beta-cells by both proximal and distal regulatory steps in stimulus-secretion coupling. Diabetes 47:57-65.

[511] Shibasaki, T., Sunaga, Y., Fujimoto, K., Kashima, Y., Seino, S., 2004. Interaction of ATP sensor, CAMP sensor, $\mathrm{Ca}^{2+}$ sensor, and voltage-dependent $\mathrm{Ca}^{2+}$ channel in insulin granule exocytosis. Journal of Biological Chemistry 279:7956-7961.

[512] Fujimoto, K., Shibasaki, T., Yokoi, N., Kashima, Y., Matsumoto, M., Sasaki, T., et al., 2002. Piccolo, a $\mathrm{Ca}^{2+}$ sensor in pancreatic beta-cells. Involvement of CAMP-GEFII.Rim2. Piccolo complex in CAMP-dependent exocytosis. Journal of Biological Chemistry 277:50497-50502.

[513] Ozaki, N., Shibasaki, T., Kashima, Y., Miki, T., Takahashi, K., Ueno, H., et al., 2000. CAMP-GEFII is a direct target of CAMP in regulated exocytosis. Nature Cell Biology 2:805-811.

[514] Shigeto, M., Ramracheya, R., Tarasov, A.I., Cha, C.Y., Chibalina, M.V., Hastoy, B., et al., 2015. GLP-1 stimulates insulin secretion by PKC-dependent TRPM4 and TRPM5 activation. Journal of Clinical Investigation 125:47144728.

[515] Murao, N., Yokoi, N., Honda, K., Han, G., Hayami, T., Gheni, G., et al., 2017. Essential roles of aspartate aminotransferase 1 and vesicular glutamate transporters in beta-cell glutamate signaling for incretin-induced insulin secretion. PLoS One 12:e0187213.

[516] Creutzfeldt, W.O., Kleine, N., Willms, B., Orskov, C., Holst, J.J., Nauck, M.A., 1996. Glucagonostatic actions and reduction of fasting hyperglycemia by exogenous glucagon-like peptide I(7-36) amide in type I diabetic patients. Diabetes Care 19:580-586.

[517] Gutniak, M., Orskov, C., Holst, J.J., Ahren, B., Efendic, S., 1992. Antidiabetogenic effect of glucagon-like peptide-1 (7-36)amide in normal subjects and patients with diabetes mellitus. New England Journal of Medicine 326:1316-1322.

[518] Krieger, J.P., Arnold, M., Pettersen, K.G., Lossel, P., Langhans, W., Lee, S.J., 2016. Knockdown of GLP-1 receptors in vagal afferents affects normal food intake and glycemia. Diabetes 65:34-43.

[519] Grasset, E., Puel, A., Charpentier, J., Collet, X., Christensen, J.E., Terce, F., et al., 2017. A specific gut microbiota dysbiosis of type 2 diabetic mice induces GLP-1 resistance through an enteric NO-dependent and gut-brain Axis mechanism. Cell Metabolism 25:1075-1090 e1075.
[520] Varin, E.M., Mulvihill, E.E., Baggio, L.L., Koehler, J.A., Cao, X., Seeley, R.J., et al., 2019. Distinct neural sites of GLP-1r expression mediate physiological versus pharmacological control of incretin action. Cell Reports 27:33713384 e3373.

[521] Hay, C.W., Sinclair, E.M., Bermano, G., Durward, E., Tadayyon, M., Docherty, K., 2005. Glucagon-like peptide-1 stimulates human insulin promoter activity in part through CAMP-responsive elements that lie upstream and downstream of the transcription start site. Journal of Endocrinology 186: $353-365$.

[522] Hani, E.H., Stoffers, D.A., Chevre, J.C., Durand, E., Stanojevic, V., Dina, C., et al., 1999. Defective mutations in the insulin promoter factor-1 (IPF-1) gene in late-onset type 2 diabetes mellitus. Journal of Clinical Investigation 104: R41-R48.

[523] Macfarlane, W.M., Frayling, T.M., Ellard, S., Evans, J.C., Allen, L.I., Bulman, M.P., et al., 1999. Missense mutations in the insulin promoter factor-1 gene predispose to type 2 diabetes. Journal of Clinical Investigation 104:R33-R39.

[524] Wang, X., Cahill, C.M., Pineyro, M.A., Zhou, J., Doyle, M.E., Egan, J.M., 1999. Glucagon-like peptide-1 regulates the beta cell transcription factor, PDX-1, in insulinoma cells. Endocrinology 140:4904-4907.

[525] Fujitani, Y., 2018. How does glucagon-like peptide 1 stimulate human betacell proliferation? A lesson from islet graft experiments. Journal of Diabetes Investigation 9:1255-1257.

[526] Butler, A.E., Janson, J., Bonner-Weir, S., Ritzel, R., Rizza, R.A., Butler, P.C., 2003. Beta-cell deficit and increased beta-cell apoptosis in humans with type 2 diabetes. Diabetes 52:102-110.

[527] Maclean, N., Ogilvie, R.F., 1955. Quantitative estimation of the pancreatic islet tissue in diabetic subjects. Diabetes 4:367-376.

[528] Rahier, J., Guiot, Y., Goebbels, R.M., Sempoux, C., Henquin, J.C., 2008. Pancreatic beta-cell mass in European subjects with type 2 diabetes. Diabetes Obesity and Metabolism 10(Suppl. 4):32-42.

[529] Saito, K., Takahashi, T., Yaginuma, N., Iwama, N., 1978. Islet morphometry in the diabetic pancreas of man. Tohoku Journal of Experimental Medicine 125:185-197.

[530] Westermark, P., Wilander, E., 1978. The influence of amyloid deposits on the islet volume in maturity onset diabetes mellitus. Diabetologia 15:417-421.

[531] Butler, P.C., Meier, J.J., Butler, A.E., Bhushan, A., 2007. The replication of beta cells in normal physiology, in disease and for therapy. Nature Clinical Practice Endocrinology and Metabolism 3:758-768.

[532] Cnop, M., Hughes, S.J., Igoillo-Esteve, M., Hoppa, M.B., Sayyed, F., van de Laar, L., et al., 2010. The long lifespan and low turnover of human islet beta cells estimated by mathematical modelling of lipofuscin accumulation. Diabetologia 53:321-330.

[533] Meier, J.J., Butler, A.E., Saisho, Y., Monchamp, T., Galasso, R., Bhushan, A., et al., 2008. Beta-cell replication is the primary mechanism subserving the postnatal expansion of beta-cell mass in humans. Diabetes 57:1584-1594.

[534] Chen, H., Gu, X., Liu, Y., Wang, J., Wirt, S.E., Bottino, R., et al., 2011. PDGF signalling controls age-dependent proliferation in pancreatic beta-cells. Nature 478:349-355.

[535] Chen, H., Gu, X., Su, I.H., Bottino, R., Contreras, J.L., Tarakhovsky, A., et al., 2009. Polycomb protein Ezh2 regulates pancreatic beta-cell Ink4a/Arf expression and regeneration in diabetes mellitus. Genes and Development 23:975-985.

[536] Gregg, B.E., Moore, P.C., Demozay, D., Hall, B.A., Li, M., Husain, A., et al., 2012. Formation of a human beta-cell population within pancreatic islets is set early in life. The Journal of Cinical Endocrinology and Metabolism 97: 3197-3206.

[537] Teta, M., Long, S.Y., Wartschow, L.M., Rankin, M.M., Kushner, J.A., 2005. Very slow turnover of beta-cells in aged adult mice. Diabetes 54:25572567. 
[538] Bonner-Weir, S., 2001. beta-cell turnover: its assessment and implications. Diabetes 50(Suppl. 1):S20-S24.

[539] Cinti, F., Bouchi, R., Kim-Muller, J.Y., Ohmura, Y., Sandoval, P.R., Masini, M., et al., 2016. Evidence of beta-cell dedifferentiation in human type 2 diabetes. The Journal of Cinical Endocrinology and Metabolism 101:1044-1054.

[540] Arakawa, M., Ebato, C., Mita, T., Hirose, T., Kawamori, R., Fujitani, Y., et al., 2009. Effects of exendin-4 on glucose tolerance, insulin secretion, and betacell proliferation depend on treatment dose, treatment duration and meal contents. Biochemical and Biophysical Research Communications 390:809814.

[541] Kawamori, D., Shirakawa, J., Liew, C.W., Hu, J., Morioka, T., Duttaroy, A., et al., 2017. GLP-1 signalling compensates for impaired insulin signalling in regulating beta cell proliferation in betalRK0 mice. Diabetologia 60:14421453.

[542] Li, Y., Cao, X., Li, L.X., Brubaker, P.L., Edlund, H., Drucker, D.J., 2005. betaCell Pdx1 expression is essential for the glucoregulatory, proliferative, and cytoprotective actions of glucagon-like peptide-1. Diabetes 54:482-491.

[543] Li, Y., Hansotia, T., Yusta, B., Ris, F., Halban, P.A., Drucker, D.J., 2003. Glucagon-like peptide-1 receptor signaling modulates beta cell apoptosis. Journal of Biological Chemistry 278:471-478.

[544] Park, S., Dong, X., Fisher, T.L., Dunn, S., Omer, A.K., Weir, G., et al., 2006. Exendin-4 uses Irs2 signaling to mediate pancreatic beta cell growth and function. Journal of Biological Chemistry 281:1159-1168.

[545] Buteau, J., El-Assaad, W., Rhodes, C.J., Rosenberg, L., Joly, E., Prentki, M., 2004. Glucagon-like peptide-1 prevents beta cell glucolipotoxicity. Diabetologia 47:806-815.

[546] Jhala, U.S., Canettieri, G., Screaton, R.A., Kulkarni, R.N., Krajewski, S., Reed, J., et al., 2003. cAMP promotes pancreatic beta-cell survival via CREBmediated induction of IRS2. Genes and Development 17:1575-1580.

[547] White, M.F., 2003. Insulin signaling in health and disease. Science 302: $1710-1711$.

[548] Tschen, S.I., Dhawan, S., Gurlo, T., Bhushan, A., 2009. Age-dependent decline in beta-cell proliferation restricts the capacity of beta-cell regeneration in mice. Diabetes 58:1312-1320.

[549] Smits, M.M., Tonneijck, L., Muskiet, M.H., Kramer, M.H., Pieters-van den Bos, I.C., Vendrik, K.E., et al., 2017. Pancreatic effects of liraglutide or sitagliptin in overweight patients with type 2 diabetes: a 12-week randomized, placebo-controlled trial. Diabetes Care 40:301-308.

[550] Kapodistria, K., Tsilibary, E.P., Kotsopoulou, E., Moustardas, P., Kitsiou, P., 2018. Liraglutide, a human glucagon-like peptide-1 analogue, stimulates AKT-dependent survival signalling and inhibits pancreatic beta-cell apoptosis. Journal of Cellular and Molecular Medicine 22:2970-2980.

[551] Sun, L., Dai, Y., Wang, C., Chu, Y., Su, X., Yang, J., et al., 2015. Novel pentapeptide GLP-1 (32-36) amide inhibits beta-cell apoptosis in vitro and improves glucose disposal in streptozotocin-induced diabetic mice. Chemical Biology and Drug Design 86:1482-1490.

[552] Fang, Y., Jiang, D., Wang, Y., Wang, Q., Lv, D., Liu, J., et al., 2018. Neuroprotection of rhGLP-1 in diabetic rats with cerebral ischemia/reperfusion injury via regulation of oxidative stress, EAAT2, and apoptosis. Drug Development Research 79:249-259.

[553] Li, D., Shang, Y., Shen, C., Li, L., Zhao, D., Ma, L., et al., 2018. Effects of Exendin-4 on pancreatic islets function in treating hyperglycemia post severe scald injury in rats. Journal of Trauma Acute Care Surgery 85:1072-1080.

[554] Khalilnezhad, A., Taskiran, D., 2018. Protective effects of glucagon-like peptide-1 (GLP-1) analogue exenatide against glucose and fructoseinduced neurotoxicity. International Journal of Neuroscience, 1-24.

[555] Liao, P., Yang, D., Liu, D., Zheng, Y., 2017. GLP-1 and ghrelin attenuate high glucose/high lipid-induced apoptosis and senescence of human microvascular endothelial cells. Cellular Physiology and Biochemistry 44:1842-1855.
[556] Farilla, L., Bulotta, A., Hirshberg, B., Li Calzi, S., Khoury, N., Noushmehr, H., et al., 2003. Glucagon-like peptide 1 inhibits cell apoptosis and improves glucose responsiveness of freshly isolated human islets. Endocrinology 144: 5149-5158.

[557] Dai, C., Hang, Y., Shostak, A., Poffenberger, G., Hart, N., Prasad, N., et al., 2017. Age-dependent human beta cell proliferation induced by glucagon-like peptide 1 and calcineurin signaling. Journal of Clinical Investigation 127: 3835-3844.

[558] Simonis-Bik, A.M., Eekhoff, E.M., de Moor, M.H., Kramer, M.H., Boomsma, D.I., Heine, R.J., et al., 2009. Genetic influences on the insulin response of the beta cell to different secretagogues. Diabetologia 52:25702577.

[559] Schafer, S.A., Tschritter, O., Machicao, F., Thamer, C., Stefan, N., Gallwitz, B., et al., 2007. Impaired glucagon-like peptide-1-induced insulin secretion in carriers of transcription factor 7-like 2 (TCF7L2) gene polymorphisms. Diabetologia 50:2443-2450.

[560] Lyssenko, V., Lupi, R., Marchetti, P., Del Guerra, S., Orho-Melander, M., Almgren, P., et al., 2007. Mechanisms by which common variants in the TCF7L2 gene increase risk of type 2 diabetes. Journal of Clinical Investigation 117:2155-2163.

[561] Pilgaard, K., Jensen, C.B., Schou, J.H., Lyssenko, V., Wegner, L., Brons, C. et al., 2009. The T allele of rs7903146 TCF7L2 is associated with impaired insulinotropic action of incretin hormones, reduced $24 \mathrm{~h}$ profiles of plasma insulin and glucagon, and increased hepatic glucose production in young healthy men. Diabetologia 52:1298-1307.

[562] Villareal, D.T., Robertson, H., Bell, G.I., Patterson, B.W., Tran, H., Wice, B., et al., 2010. TCF7L2 variant rs7903146 affects the risk of type 2 diabetes by modulating incretin action. Diabetes 59:479-485.

[563] Sathananthan, A., Man, C.D., Micheletto, F., Zinsmeister, A.R., Camilleri, M., Giesler, P.D., et al., 2010. Common genetic variation in GLP1R and insulin secretion in response to exogenous GLP-1 in nondiabetic subjects: a pilot study. Diabetes Care 33:2074-2076.

[564] Schafer, S.A., Mussig, K., Staiger, H., Machicao, F., Stefan, N., Gallwitz, B., et al., 2009. A common genetic variant in WFS1 determines impaired glucagon-like peptide-1-induced insulin secretion. Diabetologia 52:10751082.

[565] t Hart, L.M., Fritsche, A., Nijpels, G., van Leeuwen, N., Donnelly, L.A., Dekker, J.M., et al., 2013. The CTRB1/2 locus affects diabetes susceptibility and treatment via the incretin pathway. Diabetes 62:3275-3281.

[566] Mussig, K., Staiger, H., Machicao, F., Haring, H.U., Fritsche, A., 2010. Genetic variants affecting incretin sensitivity and incretin secretion. Diabetologia 53: 2289-2297.

[567] Ordelheide, A.M., Gerst, F., Rothfuss, O., Heni, M., Haas, C., Thielker, I., et al., 2013. Nor-1, a novel incretin-responsive regulator of insulin genes and insulin secretion. Molecular Metabolism 2:243-255.

[568] Kirchhoff, K., Machicao, F., Haupt, A., Schafer, S.A., Tschritter, 0., Staiger, H., et al., 2008. Polymorphisms in the TCF7L2, CDKAL1 and SLC30A8 genes are associated with impaired proinsulin conversion. Diabetologia 51:597-601.

[569] Shu, L., Matveyenko, A.V., Kerr-Conte, J., Cho, J.H., Mclntosh, C.H., Maedler, K., 2009. Decreased TCF7L2 protein levels in type 2 diabetes mellitus correlate with downregulation of GIP- and GLP-1 receptors and impaired beta-cell function. Human Molecular Genetics 18:2388-2399.

[570] Gudmundsdottir, V., Pedersen, H.K., Allebrandt, K.V., Brorsson, C., van Leeuwen, N., Banasik, K., et al., 2018. Integrative network analysis highlights biological processes underlying GLP-1 stimulated insulin secretion: a DIRECT study. PLoS One 13:e0189886.

[571] Herzberg-Schafer, S., Heni, M., Stefan, N., Haring, H.U., Fritsche, A., 2012. Impairment of GLP1-induced insulin secretion: role of genetic background, 
insulin resistance and hyperglycaemia. Diabetes Obesity and Metabolism 14(Suppl. 3):85-90.

[572] Heni, M., Ketterer, C., Thamer, C., Herzberg-Schafer, S.A., Guthoff, M., Stefan, N., et al., 2010. Glycemia determines the effect of type 2 diabetes risk genes on insulin secretion. Diabetes 59:3247-3252.

[573] Willms, B., Werner, J., Holst, J.J., Orskov, C., Creutzfeldt, W., Nauck, M.A., 1996. Gastric emptying, glucose responses, and insulin secretion after a liquid test meal: effects of exogenous glucagon-like peptide-1 (GLP-1)-(7-36) amide in type 2 (noninsulin-dependent) diabetic patients. The Journal of Cinical Endocrinology and Metabolism 81:327-332.

[574] Drucker, D.J., 2018. Mechanisms of action and therapeutic application of glucagon-like peptide-1. Cell Metabolism 27:740-756.

[575] Fridolf, T., Bottcher, G., Sundler, F., Ahren, B., 1991. GLP-1 and GLP-1(7-36) amide: influences on basal and stimulated insulin and glucagon secretion in the mouse. Pancreas 6:208-215.

[576] Freyse, E.J., Becher, T., El-Hag, O., Knospe, S., Goke, B., Fischer, U., 1997. Blood glucose lowering and glucagonostatic effects of glucagon-like peptide I in insulin-deprived diabetic dogs. Diabetes 46:824-828.

[577] Hare, K.J., Knop, F.K., Asmar, M., Madsbad, S., Deacon, C.F., Holst, J.J., et al., 2009. Preserved inhibitory potency of GLP-1 on glucagon secretion in type 2 diabetes mellitus. The Journal of Cinical Endocrinology and Metabolism 94:4679-4687.

[578] Hare, K.J., Vilsboll, T., Asmar, M., Deacon, C.F., Knop, F.K., Holst, J.J., 2010. The glucagonostatic and insulinotropic effects of glucagon-like peptide 1 contribute equally to its glucose-lowering action. Diabetes 59:1765-1770.

[579] Hvidberg, A., Nielsen, M.T., Hilsted, J., Orskov, C., Holst, J.J., 1994. Effect of glucagon-like peptide-1 (proglucagon 78-107amide) on hepatic glucose production in healthy man. Metabolism 43:104-108.

[580] Junker, A.E., Gluud, L.L., van Hall, G., Holst, J.J., Knop, F.K., Vilsboll, T., 2016. Effects of glucagon-like peptide-1 on glucagon secretion in patients with non-alcoholic fatty liver disease. Journal of Hepatology 64: 908-915.

[581] Plamboeck, A., Veedfald, S., Deacon, C.F., Hartmann, B., Vilsboll, T., Knop, F.K., et al., 2015. The role of efferent cholinergic transmission for the insulinotropic and glucagonostatic effects of GLP-1. American Journal of Physiology Regulatory Integrative and Comparative Physiology 309:R544R551.

[582] de Heer, J., Rasmussen, C., Coy, D.H., Holst, J.J., 2008. Glucagon-like peptide-1, but not glucose-dependent insulinotropic peptide, inhibits glucagon secretion via somatostatin (receptor subtype 2) in the perfused rat pancreas. Diabetologia 51:2263-2270.

[583] De Marinis, Y.Z., Salehi, A., Ward, C.E., Zhang, Q., Abdulkader, F., Bengtsson, M., et al., 2010. GLP-1 inhibits and adrenaline stimulates glucagon release by differential modulation of $\mathrm{N}$ - and L-type $\mathrm{Ca}^{2+}$ channeldependent exocytosis. Cell Metabolism 11:543-553.

[584] Gerich, J.E., Lorenzi, M., Schneider, V., Karam, J.H., Rivier, J., Guillemin, R., et al., 1974. Effects of somatostatin on plasma glucose and glucagon levels in human diabetes mellitus. Pathophysiologic and therapeutic implications. New England Journal of Medicine 291:544-547.

[585] Barden, N., Cote, J.P., Lavoie, M., Dupont, A., 1978. Secretion of somatostatin by rat islets of Langerhans and gastric mucosa and a role for pancreatic somatostatin in the regulation of glucagon release. Metabolism 27:1215-1218.

[586] Barden, N., Lavoie, M., Dupont, A., Cote, J., Cote, J.P., 1977. Stimulation of glucagon release by addition of anti-stomatostatin serum to islets of Langerhans in vitro. Endocrinology 101:635-638.

[587] Kaneko, K., Shirotani, T., Araki, E., Matsumoto, K., Taguchi, T., Motoshima, H., et al., 1999. Insulin inhibits glucagon secretion by the activation of PI3-kinase in In-R1-G9 cells. Diabetes Research and Clinical Practice 44:83-92.
[588] Xu, E., Kumar, M., Zhang, Y., Ju, W., Obata, T., Zhang, N., et al., 2006. Intraislet insulin suppresses glucagon release via GABA-GABAA receptor system. Cell Metabolism 3:47-58.

[589] Wendt, A., Birnir, B., Buschard, K., Gromada, J., Salehi, A., Sewing, S., et al., 2004. Glucose inhibition of glucagon secretion from rat alpha-cells is mediated by GABA released from neighboring beta-cells. Diabetes 53:10381045.

[590] Blundell, T.L., Cutfield, J.F., Dodson, E.J., Dodson, G.G., Hodgkin, D.C., Mercola, D.A., 1972. The crystal structure of rhombohedral 2 zinc insulin. Cold Spring Harbor Symposia on Quantitative Biology 36:233-241.

[591] Blundell, T.L., Cutfield, J.F., Dodson, G.G., Dodson, E., Hodgkin, D.C., Mercola, D., 1971. The structure and biology of insulin. Biochemical Journal 125:50P-51P.

[592] Gee, K.R., Zhou, Z.L., Qian, W.J., Kennedy, R., 2002. Detection and imaging of zinc secretion from pancreatic beta-cells using a new fluorescent zinc indicator. Journal of the American Chemical Society 124:776-778.

[593] Franklin, I., Gromada, J., Gjinovci, A., Theander, S., Wollheim, C.B., 2005. Beta-cell secretory products activate alpha-cell ATP-dependent potassium channels to inhibit glucagon release. Diabetes 54:1808-1815.

[594] Ishihara, H., Maechler, P., Gjinovci, A., Herrera, P.L., Wollheim, C.B., 2003. Islet beta-cell secretion determines glucagon release from neighbouring alpha-cells. Nature Cell Biology 5:330-335.

[595] Zhou, H., Zhang, T., Harmon, J.S., Bryan, J., Robertson, R.P., 2007. Zinc, not insulin, regulates the rat alpha-cell response to hypoglycemia in vivo. Diabetes $56: 1107-1112$.

[596] Gyulkhandanyan, A.V., Lee, S.C., Bikopoulos, G., Dai, F., Wheeler, M.B., 2006. The Zn2+-transporting pathways in pancreatic beta-cells: a role for the L-type voltage-gated $\mathrm{Ca}^{2+}$ channel. Journal of Biological Chemistry 281: 9361-9372.

[597] Gedulin, B.R., Rink, T.J., Young, A.A., 1997. Dose-response for glucagonostatic effect of amylin in rats. Metabolism 46:67-70.

[598] Gedulin, B.R., Jodka, C.M., Herrmann, K., Young, A.A., 2006. Role of endogenous amylin in glucagon secretion and gastric emptying in rats demonstrated with the selective antagonist, AC187. Regulatory Peptides 137: $121-127$.

[599] Levetan, C., Want, L.L., Weyer, C., Strobel, S.A., Crean, J., Wang, Y., et al., 2003. Impact of pramlintide on glucose fluctuations and postprandial glucose, glucagon, and triglyceride excursions among patients with type 1 diabetes intensively treated with insulin pumps. Diabetes Care 26:1-8.

[600] Nyholm, B., Orskov, L., Hove, K.Y., Gravholt, C.H., Moller, N., Alberti, K.G., et al., 1999. The amylin analog pramlintide improves glycemic control and reduces postprandial glucagon concentrations in patients with type 1 diabetes mellitus. Metabolism 48:935-941.

[601] Broderick, C.L., Brooke, G.S., DiMarchi, R.D., Gold, G., 1991. Human and rat amylin have no effects on insulin secretion in isolated rat pancreatic islets. Biochemical and Biophysical Research Communications 177:932-938.

[602] Inoue, K., Hiramatsu, S., Hisatomi, A., Umeda, F., Nawata, H., 1993. Effects of amylin on the release of insulin and glucagon from the perfused rat pancreas. Hormone and Metabolic Research 25:135-137.

[603] Silvestre, R.A., Peiro, E., Degano, P., Miralles, P., Marco, J., 1990. Inhibitory effect of rat amylin on the insulin responses to glucose and arginine in the perfused rat pancreas. Regulatory Peptides 31:23-31.

[604] Ding, W.G., Renstrom, E., Rorsman, P., Buschard, K., Gromada, J., 1997. Glucagon-like peptide I and glucose-dependent insulinotropic polypeptide stimulate $\mathrm{Ca}^{2+}$-induced secretion in rat alpha-cells by a protein kinase $\mathrm{A}$ mediated mechanism. Diabetes 46:792-800.

[605] Dunning, B.E., Foley, J.E., Ahren, B., 2005. Alpha cell function in health and disease: influence of glucagon-like peptide-1. Diabetologia 48:1700-1713.

[606] Ma, X., Zhang, Y., Gromada, J., Sewing, S., Berggren, P.O., Buschard, K., et al., 2005. Glucagon stimulates exocytosis in mouse and rat pancreatic 
alpha-cells by binding to glucagon receptors. Molecular Endocrinology 19: $198-212$.

[607] Zhang, Y., Parajuli, K.R., Fava, G.E., Gupta, R., Xu, W., Nguyen, L.U., et al., 2019. GLP-1 receptor in pancreatic alpha-cells regulates glucagon secretion in a glucose-dependent bidirectional manner. Diabetes 68:34-44.

[608] Ramracheya, R., Chapman, C., Chibalina, M., Dou, H., Miranda, C., Gonzalez, A., et al., 2018. GLP-1 suppresses glucagon secretion in human pancreatic alpha-cells by inhibition of $\mathrm{P} / \mathrm{Q}$-type $\mathrm{Ca}(2+)$ channels. Physics Reports 6:e13852.

[609] Wei, Y., Mojsov, S., 1996. Distribution of GLP-1 and PACAP receptors in human tissues. Acta Physiologica Scandinavica 157:355-357.

[610] Bhashyam, S., Fields, A.V., Patterson, B., Testani, J.M., Chen, L., Shen, Y.T., et al., 2010. Glucagon-like peptide-1 increases myocardial glucose uptake via p38alpha MAP kinase-mediated, nitric oxide-dependent mechanisms in conscious dogs with dilated cardiomyopathy. Circulatory Heart Failure 3: $512-521$.

[611] Sivertsen, J., Rosenmeier, J., Holst, J.J., Vilsboll, T., 2012. The effect of glucagon-like peptide 1 on cardiovascular risk. Nature Reviews Cardiology 9: 209-222.

[612] Nystrom, T., Gutniak, M.K., Zhang, Q., Zhang, F., Holst, J.J., Ahren, B., et al., 2004. Effects of glucagon-like peptide-1 on endothelial function in type 2 diabetes patients with stable coronary artery disease. American Journal of Physiology Endocrinology and Metabolism 287:E1209-E1215.

[613] Baggio, L.L., Yusta, B., Mulvihill, E.E., Cao, X., Streutker, C.J., Butany, J., et al., 2018. GLP-1 receptor expression within the human heart. Endocrinology 159:1570-1584.

[614] Noyan-Ashraf, M.H., Momen, M.A., Ban, K., Sadi, A.M., Zhou, Y.Q., Riazi, A.M., et al., 2009. GLP-1R agonist liraglutide activates cytoprotective pathways and improves outcomes after experimental myocardial infarction in mice. Diabetes 58:975-983.

[615] Wang, S.X., Xie, Y., Zhou, X., Sha, W.W., Wang, W.L., Han, L.P., et al., 2010. Effect of glucagon-like peptide-1 on hypoxia-reoxygenation induced injury in neonatal rat cardiomyocytes. Zhonghua Xinxueguanbing Zazhi 38:72-75.

[616] Ceriello, A., Esposito, K., Testa, R., Bonfigli, A.R., Marra, M., Giugliano, D., 2011. The possible protective role of glucagon-like peptide 1 on endothelium during the meal and evidence for an "endothelial resistance" to glucagon-like peptide 1 in diabetes. Diabetes Care 34:697-702.

[617] Nikolaidis, L.A., Elahi, D., Hentosz, T., Doverspike, A., Huerbin, R., Zourelias, L., et al., 2004. Recombinant glucagon-like peptide-1 increases myocardial glucose uptake and improves left ventricular performance in conscious dogs with pacing-induced dilated cardiomyopathy. Circulation 110 : 955-961.

[618] Nikolaidis, L.A., Doverspike, A., Hentosz, T., Zourelias, L., Shen, Y.T., Elahi, D., et al., 2005. Glucagon-like peptide-1 limits myocardial stunning following brief coronary occlusion and reperfusion in conscious canines. Journal of Pharmacology and Experimental Therapeutics 312:303-308.

[619] Nikolaidis, L.A., Mankad, S., Sokos, G.G., Miske, G., Shah, A., Elahi, D., et al., 2004. Effects of glucagon-like peptide- 1 in patients with acute myocardial infarction and left ventricular dysfunction after successful reperfusion. Circulation 109:962-965.

[620] Sun, F., Wu, S., Guo, S., Yu, K., Yang, Z., Li, L., et al., 2015. Impact of GLP-1 receptor agonists on blood pressure, heart rate and hypertension among patients with type 2 diabetes: a systematic review and network meta-analysis. Diabetes Research and Clinical Practice 110:26-37.

[621] Marso, S.P., Daniels, G.H., Brown-Frandsen, K., Kristensen, P., Mann, J.F., Nauck, M.A., et al., 2016. Liraglutide and cardiovascular outcomes in type 2 diabetes. New England Journal of Medicine 375:311-322.

[622] Ussher, J.R., Drucker, D.J., 2012. Cardiovascular biology of the incretin system. Endocrine Reviews 33:187-215.
[623] Ceriello, A., De Nigris, V., Pujadas, G., La Sala, L., Bonfigli, A.R., Testa, R., et al., 2016. The simultaneous control of hyperglycemia and GLP-1 infusion normalize endothelial function in type 1 diabetes. Diabetes Research and Clinical Practice 114:64-68.

[624] Green, B.D., Hand, K.V., Dougan, J.E., McDonnell, B.M., Cassidy, R.S., Grieve, D.J., 2008. GLP-1 and related peptides cause concentrationdependent relaxation of rat aorta through a pathway involving KATP and CAMP. Archives of Biochemistry and Biophysics 478:136-142.

[625] Bose, A.K., Mocanu, M.M., Carr, R.D., Brand, C.L., Yellon, D.M., 2005. Glucagon-like peptide 1 can directly protect the heart against ischemia/ reperfusion injury. Diabetes 54:146-151.

[626] Sonne, D.P., Engstrom, T., Treiman, M., 2008. Protective effects of GLP-1 analogues exendin-4 and GLP-1(9-36) amide against ischemia-reperfusion injury in rat heart. Regulatory Peptides 146:243-249.

[627] Vila Petroff, M.G., Egan, J.M., Wang, X., Sollott, S.J., 2001. Glucagon-like peptide-1 increases CAMP but fails to augment contraction in adult rat cardiac myocytes. Circulation Research 89:445-452.

[628] Zhao, T., Parikh, P., Bhashyam, S., Bolukoglu, H., Poornima, I., Shen, Y.T., et al., 2006. Direct effects of glucagon-like peptide-1 on myocardial contractility and glucose uptake in normal and postischemic isolated rat hearts. Journal of Pharmacology and Experimental Therapeutics 317:11061113.

[629] Gros, R., You, X., Baggio, L.L., Kabir, M.G., Sadi, A.M., Mungrue, I.N., et al., 2003. Cardiac function in mice lacking the glucagon-like peptide-1 receptor. Endocrinology 144:2242-2252.

[630] Ussher, J.R., Baggio, L.L., Campbell, J.E., Mulvihill, E.E., Kim, M., Kabir, M.G., et al., 2014. Inactivation of the cardiomyocyte glucagon-like peptide-1 receptor (GLP-1R) unmasks cardiomyocyte-independent GLP-1Rmediated cardioprotection. Molecular Metabolism 3:507-517.

[631] Ban, K., Kim, K.H., Cho, C.K., Sauve, M., Diamandis, E.P., Backx, P.H., et al., 2010. Glucagon-like peptide (GLP)-1(9-36)amide-mediated cytoprotection is blocked by exendin(9-39) yet does not require the known GLP-1 receptor. Endocrinology 151:1520-1531.

[632] Rose, B.A., Force, T., Wang, Y., 2010. Mitogen-activated protein kinase signaling in the heart: angels versus demons in a heart-breaking tale. Physiological Reviews 90:1507-1546.

[633] Sussman, M.A., Volkers, M., Fischer, K., Bailey, B., Cottage, C.T., Din, S. et al., 2011. Myocardial AKT: the omnipresent nexus. Physiological Reviews 91:1023-1070.

[634] Barragan, J.M., Rodriguez, R.E., Blazquez, E., 1994. Changes in arterial blood pressure and heart rate induced by glucagon-like peptide-1-(7-36) amide in rats. American Journal of Physiology 266:E459-E466.

[635] Barragan, J.M., Rodriguez, R.E., Eng, J., Blazquez, E., 1996. Interactions of exendin-(9-39) with the effects of glucagon-like peptide-1-(7-36) amide and of exendin- 4 on arterial blood pressure and heart rate in rats. Regulatory Peptides 67:63-68.

[636] Barragan, J.M., Eng, J., Rodriguez, R., Blazquez, E., 1999. Neural contribution to the effect of glucagon-like peptide-1-(7-36) amide on arterial blood pressure in rats. American Journal of Physiology 277:E784-E791.

[637] Griffioen, K.J., Wan, R., Okun, E., Wang, X., Lovett-Barr, M.R., Li, Y., et al., 2011. GLP-1 receptor stimulation depresses heart rate variability and inhibits neurotransmission to cardiac vagal neurons. Cardiovascular Research 89: $72-78$.

[638] Yamamoto, H., Lee, C.E., Marcus, J.N., Williams, T.D., Overton, J.M., Lopez, M.E., et al., 2002. Glucagon-like peptide-1 receptor stimulation increases blood pressure and heart rate and activates autonomic regulatory neurons. Journal of Clinical Investigation 110:43-52.

[639] Gardiner, S.M., March, J.E., Kemp, P.A., Bennett, T., 2006. Mesenteric vasoconstriction and hindquarters vasodilatation accompany the pressor 
actions of exendin- 4 in conscious rats. Journal of Pharmacology and Experimental Therapeutics 316:852-859.

[640] Kim, M., Platt, M.J., Shibasaki, T., Quaggin, S.E., Backx, P.H., Seino, S., et al., 2013. GLP-1 receptor activation and Epac2 link atrial natriuretic peptide secretion to control of blood pressure. Nature Medicine 19:567-575.

[641] Hirata, K., Kume, S., Araki, S., Sakaguchi, M., Chin-Kanasaki, M., Isshiki, K., et al., 2009. Exendin-4 has an anti-hypertensive effect in salt-sensitive mice model. Biochemical and Biophysical Research Communications 380:44-49.

[642] Yu, M., Moreno, C., Hoagland, K.M., Dahly, A., Ditter, K., Mistry, M., et al., 2003. Antihypertensive effect of glucagon-like peptide 1 in Dahl salt-sensitive rats. Journal of Hypertension 21:1125-1135.

[643] Edwards, C.M., Edwards, A.V., Bloom, S.R., 1997. Cardiovascular and pancreatic endocrine responses to glucagon-like peptide-1(7-36) amide in the conscious calf. Experimental Physiology 82:709-716.

[644] Bremholm, L., Andersen, U.B., Hornum, M., Hilsted, L., Veedfald, S., Hartmann, B., et al., 2017. Acute effects of glucagon-like peptide-1, GLP-1936 amide, and exenatide on mesenteric blood flow, cardiovascular parameters, and biomarkers in healthy volunteers. Physics Reports 5.

[645] Drucker, D.J., Nauck, M.A., 2006. The incretin system: glucagon-like peptide- 1 receptor agonists and dipeptidyl peptidase- 4 inhibitors in type 2 diabetes. Lancet 368:1696-1705.

[646] Kumarathurai, P., Anholm, C., Fabricius-Bjerre, A., Nielsen, O.W., Kristiansen, 0., Madsbad, S., et al., 2017. Effects of the glucagon-like peptide-1 receptor agonist liraglutide on 24-h ambulatory blood pressure in patients with type 2 diabetes and stable coronary artery disease: a randomized, double-blind, placebo-controlled, crossover study. Journal of Hypertension 35:1070-1078.

[647] Kumarathurai, P., Anholm, C., Larsen, B.S., Olsen, R.H., Madsbad, S., Kristiansen, 0., et al., 2017. Effects of liraglutide on heart rate and heart rate variability: a randomized, double-blind, placebo-controlled crossover study. Diabetes Care 40:117-124.

[648] Srivastava, G., Apovian, C.M., 2018. Current pharmacotherapy for obesity. Nature Reviews Endocrinology 14:12-24.

[649] Ussher, J.R., Drucker, D.J., 2014. Cardiovascular actions of incretin-based therapies. Circulation Research 114:1788-1803.

[650] Asmar, A., Asmar, M., Simonsen, L., Madsbad, S., Holst, J.J., Hartmann, B., et al., 2017. Glucagon-like peptide-1 elicits vasodilation in adipose tissue and skeletal muscle in healthy men. Physics Reports 5.

[651] Asmar, M., Arngrim, N., Simonsen, L., Asmar, A., Nordby, P., Holst, J.J., et al., 2016. The blunted effect of glucose-dependent insulinotropic polypeptide in subcutaneous abdominal adipose tissue in obese subjects is partly reversed by weight loss. Nutrition and Diabetes 6:e208.

[652] Asmar, M., Simonsen, L., Arngrim, N., Holst, J.J., Dela, F., Bulow, J., 2014. Glucose-dependent insulinotropic polypeptide has impaired effect on abdominal, subcutaneous adipose tissue metabolism in obese subjects. International Journal of Obesity (London) 38:259-265.

[653] Asmar, M., Simonsen, L., Asmar, A., Holst, J.J., Dela, F., Bulow, J., 2016. Insulin plays a permissive role for the vasoactive effect of GIP regulating adipose tissue metabolism in humans. The Journal of Cinical Endocrinology and Metabolism 101:3155-3162.

[654] Asmar, M., Simonsen, L., Madsbad, S., Stallknecht, B., Holst, J.J., Bulow, J., 2010. Glucose-dependent insulinotropic polypeptide may enhance fatty acid re-esterification in subcutaneous abdominal adipose tissue in lean humans. Diabetes 59:2160-2163.

[655] Mannucci, E., Monami, M., 2017. Cardiovascular safety of incretin-based therapies in type 2 diabetes: systematic review of integrated analyses and randomized controlled trials. Advances in Therapy 34:1-40.

[656] Chai, W., Dong, Z., Wang, N., Wang, W., Tao, L., Cao, W., et al., 2012. Glucagon-like peptide 1 recruits microvasculature and increases glucose use in muscle via a nitric oxide-dependent mechanism. Diabetes 61:888-896.
[657] Asmar, A., Cramon, P.K., Simonsen, L., Asmar, M., Sorensen, C.M. Madsbad, S., et al., 2019. Extracellular fluid volume expansion uncovers a natriuretic action of GLP-1: a functional GLP-1-renal axis in man. The Journal of Cinical Endocrinology and Metabolism.

[658] Guyton, A.C., Coleman, T.G., Granger, H.J., 1972. Circulation: overall regulation. Annual Review of Physiology 34:13-46.

[659] Ivy, J.R., Bailey, M.A., 2014. Pressure natriuresis and the renal control of arterial blood pressure. Journal of Physiology 592:3955-3967.

[660] Crajoinas, R.O., Oricchio, F.T., Pessoa, T.D., Pacheco, B.P., Lessa, L.M., Malnic, G., et al., 2011. Mechanisms mediating the diuretic and natriuretic actions of the incretin hormone glucagon-like peptide-1. American Journal of Physiology Renal Physiology 301:F355-F363.

[661] Gutzwiller, J.P., Tschopp, S., Bock, A., Zehnder, C.E., Huber, A.R., Kreyenbuehl, M., et al., 2004. Glucagon-like peptide 1 induces natriuresis in healthy subjects and in insulin-resistant obese men. The Journal of Cinical Endocrinology and Metabolism 89:3055-3061.

[662] Lovshin, J.A., Barnie, A., DeAlmeida, A., Logan, A., Zinman, B., Drucker, D.J., 2015. Liraglutide promotes natriuresis but does not increase circulating levels of atrial natriuretic peptide in hypertensive subjects with type 2 diabetes. Diabetes Care 38:132-139.

[663] Skov, J., Dejgaard, A., Frokiaer, J., Holst, J.J., Jonassen, T., Rittig, S., et al., 2013. Glucagon-like peptide-1 (GLP-1): effect on kidney hemodynamics and renin-angiotensin-aldosterone system in healthy men. The Journal of Cinical Endocrinology and Metabolism 98:E664-E671.

[664] Skov, J., Holst, J.J., Gotze, J.P., Frokiaer, J., Christiansen, J.S., 2014. Glucagon-like peptide-1: effect on pro-atrial natriuretic peptide in healthy males. Endocrine Connectivity 3:11-16.

[665] Henderson, S.J., Konkar, A., Hornigold, D.C., Trevaskis, J.L., Jackson, R., Fritsch Fredin, M., et al., 2016. Robust anti-obesity and metabolic effects of a dual GLP-1/glucagon receptor peptide agonist in rodents and non-human primates. Diabetes Obesity and Metabolism 18:1176-1190.

[666] Beiroa, D., Imbernon, M., Gallego, R., Senra, A., Herranz, D., Villarroya, F., et al., 2014. GLP-1 agonism stimulates brown adipose tissue thermogenesis and browning through hypothalamic AMPK. Diabetes 63:3346-3358.

[667] Donahey, J.C., van Dijk, G., Woods, S.C., Seeley, R.J., 1998. Intraventricular GLP-1 reduces short- but not long-term food intake or body weight in lean and obese rats. Brain Research 779:75-83.

[668] Turton, M.D., O'Shea, D., Gunn, I., Beak, S.A., Edwards, C.M., Meeran, K., et al., 1996. A role for glucagon-like peptide-1 in the central regulation of feeding. Nature 379:69-72.

[669] Furuse, M., Matsumoto, M., Okumura, J., Sugahara, K., Hasegawa, S., 1997. Intracerebroventricular injection of mammalian and chicken glucagon-like peptide-1 inhibits food intake of the neonatal chick. Brain Research 755: 167-169.

[670] Shousha, S., Nakahara, K., Nasu, T., Sakamoto, T., Murakami, N., 2007. Effect of glucagon-like peptide-1 and -2 on regulation of food intake, body temperature and locomotor activity in the Japanese quail. Neuroscience Letters 415:102-107.

[671] Raun, K., von Voss, P., Knudsen, L.B., 2007. Liraglutide, a once-daily human glucagon-like peptide-1 analog, minimizes food intake in severely obese minipigs. Obesity (Silver Spring) 15:1710-1716.

[672] Renner, S., Blutke, A., Dobenecker, B., Dhom, G., Muller, T.D., Finan, B., et al., 2018. Metabolic syndrome and extensive adipose tissue inflammation in morbidly obese Gottingen minipigs. Molecular Metabolism.

[673] Elvert, R., Bossart, M., Herling, A.W., Weiss, T., Zhang, B., Kannt, A., et al., 2018. Team players or opponents: coadministration of selective glucagon and GLP-1 receptor agonists in obese diabetic monkeys. Endocrinology 159: 3105-3119.

[674] Scott, K.A., Moran, T.H., 2007. The GLP-1 agonist exendin-4 reduces food intake in nonhuman primates through changes in meal size. American 
Journal of Physiology Regulatory Integrative and Comparative Physiology 293: R983-R987.

[675] Gutzwiller, J.P., Drewe, J., Goke, B., Schmidt, H., Rohrer, B., Lareida, J., et al., 1999. Glucagon-like peptide-1 promotes satiety and reduces food intake in patients with diabetes mellitus type 2. American Journal of Physiology 276:R1541-R1544.

[676] Gutzwiller, J.P., Goke, B., Drewe, J., Hildebrand, P., Ketterer, S., Handschin, D., et al., 1999. Glucagon-like peptide-1: a potent regulator of food intake in humans. Gut 44:81-86.

[677] Verdich, C., Flint, A., Gutzwiller, J.P., Naslund, E., Beglinger, C., Hellstrom, P.M., et al., 2001. A meta-analysis of the effect of glucagon-like peptide-1 (7-36) amide on ad libitum energy intake in humans. The Journal of Cinical Endocrinology and Metabolism 86:4382-4389.

[678] Flint, A., Raben, A., Astrup, A., Holst, J.J., 1998. Glucagon-like peptide 1 promotes satiety and suppresses energy intake in humans. Journal of Clinical Investigation 101:515-520.

[679] De Silva, A., Salem, V., Long, C.J., Makwana, A., Newbould, R.D., Rabiner, E.A., et al., 2011. The gut hormones PYY 3-36 and GLP-1 7-36 amide reduce food intake and modulate brain activity in appetite centers in humans. Cell Metabolism 14:700-706.

[680] Schlogl, H., Kabisch, S., Horstmann, A., Lohmann, G., Muller, K., Lepsien, J., et al., 2013. Exenatide-induced reduction in energy intake is associated with increase in hypothalamic connectivity. Diabetes Care 36:1933-1940.

[681] Ten Kulve, J.S., Veltman, D.J., van Bloemendaal, L., Barkhof, F., Drent, M.L., Diamant, M., et al., 2016. Liraglutide reduces CNS activation in response to visual food cues only after short-term treatment in patients with type 2 diabetes. Diabetes Care 39:214-221.

[682] van Bloemendaal, L., RG, I.J., Ten Kulve, J.S., Barkhof, F., Konrad, R.J., Drent, M.L., et al., 2014. GLP-1 receptor activation modulates appetite- and reward-related brain areas in humans. Diabetes 63:4186-4196.

[683] van Bloemendaal, L., Veltman, D.J., ten Kulve, J.S., Drent, M.L., Barkhof, F., Diamant, M., et al., 2015. Emotional eating is associated with increased brain responses to food-cues and reduced sensitivity to GLP-1 receptor activation. Obesity (Silver Spring) 23:2075-2082.

[684] van Bloemendaal, L., Veltman, D.J., Ten Kulve, J.S., Groot, P.F., Ruhe, H.G., Barkhof, F., et al., 2015. Brain reward-system activation in response to anticipation and consumption of palatable food is altered by glucagon-like peptide-1 receptor activation in humans. Diabetes Obesity and Metabolism 17:878-886.

[685] Clemmensen, C., Finan, B., Fischer, K., Tom, R.Z., Legutko, B., Sehrer, L., et al., 2015. Dual melanocortin-4 receptor and GLP-1 receptor agonism amplifies metabolic benefits in diet-induced obese mice. EMBO Molecular Medicine 7:288-298.

[686] Secher, A., Jelsing, J., Baquero, A.F., Hecksher-Sorensen, J., Cowley, M.A., Dalboge, L.S., et al., 2014. The arcuate nucleus mediates GLP-1 receptor agonist liraglutide-dependent weight loss. Journal of Clinical Investigation 124:4473-4488.

[687] Kanoski, S.E., Fortin, S.M., Arnold, M., Grill, H.J., Hayes, M.R., 2011. Peripheral and central GLP-1 receptor populations mediate the anorectic effects of peripherally administered GLP-1 receptor agonists, liraglutide and exendin4. Endocrinology 152:3103-3112.

[688] Sisley, S., Gutierrez-Aguilar, R., Scott, M., D’Alessio, D.A., Sandoval, D.A., Seeley, R.J., 2014. Neuronal GLP1R mediates liraglutide's anorectic but not glucose-lowering effect. Journal of Clinical Investigation 124:2456-2463.

[689] Swick, J.C., Alhadeff, A.L., Grill, H.J., Urrea, P., Lee, S.M., Roh, H., et al., 2015. Parabrachial nucleus contributions to glucagon-like peptide-1 receptor agonist-induced hypophagia. Neuropdsychopharmacology 40:2001-2014.

[690] Baggio, L.L., Huang, Q., Brown, T.J., Drucker, D.J., 2004. Oxyntomodulin and glucagon-like peptide-1 differentially regulate murine food intake and energy expenditure. Gastroenterology 127:546-558.
[691] Labouesse, M.A., Stadlbauer, U., Weber, E., Arnold, M., Langhans, W., Pacheco-Lopez, G., 2012. Vagal afferents mediate early satiation and prevent flavour avoidance learning in response to intraperitoneally infused exendin-4. Journal of Neuroendocrinology 24:1505-1516.

[692] Baumgartner, I., Pacheco-Lopez, G., Ruttimann, E.B., Arnold, M., Asarian, L., Langhans, W., et al., 2010. Hepatic-portal vein infusions of glucagon-like peptide- 1 reduce meal size and increase c-Fos expression in the nucleus tractus solitarii, area postrema and central nucleus of the amygdala in rats. Journal of Neuroendocrinology 22:557-563.

[693] Bucinskaite, V., Tolessa, T., Pedersen, J., Rydqvist, B., Zerihun, L., Holst, J.J., et al., 2009. Receptor-mediated activation of gastric vagal afferents by glucagon-like peptide- 1 in the rat. Neurogastroenterology and Motility 21:978-e978.

[694] Ruttimann, E.B., Arnold, M., Hillebrand, J.J., Geary, N., Langhans, W., 2009. Intrameal hepatic portal and intraperitoneal infusions of glucagon-like peptide- 1 reduce spontaneous meal size in the rat via different mechanisms. Endocrinology 150:1174-1181.

[695] Plamboeck, A., Veedfald, S., Deacon, C.F., Hartmann, B., Wettergren, A. Svendsen, L.B., et al., 2013. The effect of exogenous GLP-1 on food intake is lost in male truncally vagotomized subjects with pyloroplasty. American Journal of Physiology Gastrointestinal and Liver Physiology 304:G1117G1127.

[696] Van Dijk, G., Thiele, T.E., Donahey, J.C., Campfield, L.A., Smith, F.J., Burn, P., et al., 1996. Central infusions of leptin and GLP-1-(7-36) amide differentially stimulate c-FLI in the rat brain. American Journal of Physiology 271:R1096-R1100.

[697] Kim, D.H., D’Alessio, D.A., Woods, S.C., Seeley, R.J., 2009. The effects of GLP-1 infusion in the hepatic portal region on food intake. Regulatory Peptides 155:110-114.

[698] Larsen, P.J., Tang-Christensen, M., Jessop, D.S., 1997. Central administration of glucagon-like peptide-1 activates hypothalamic neuroendocrine neurons in the rat. Endocrinology 138:4445-4455.

[699] Alhadeff, A.L., Mergler, B.D., Zimmer, D.J., Turner, C.A., Reiner, D.J., Schmidt, H.D., et al., 2017. Endogenous glucagon-like peptide-1 receptor signaling in the nucleus tractus solitarius is required for food intake control. Neuropsychopharmacology 42:1471-1479.

[700] Barrera, J.G., Jones, K.R., Herman, J.P., D’Alessio, D.A., Woods, S.C., Seeley, R.J., 2011. Hyperphagia and increased fat accumulation in two models of chronic CNS glucagon-like peptide-1 loss of function. Journal of Neuroscience 31:3904-3913.

[701] Dickson, S.L., Shirazi, R.H., Hansson, C., Bergquist, F., Nissbrandt, H., Skibicka, K.P., 2012. The glucagon-like peptide 1 (GLP-1) analogue, exendin4, decreases the rewarding value of food: a new role for mesolimbic GLP-1 receptors. Journal of Neuroscience 32:4812-4820.

[702] Hsu, T.M., Hahn, J.D., Konanur, V.R., Lam, A., Kanoski, S.E., 2015. Hippocampal GLP-1 receptors influence food intake, meal size, and effort-based responding for food through volume transmission. Neuropsychopharmacology 40:327-337.

[703] Mietlicki-Baase, E.G., Ortinski, P.I., Rupprecht, L.E., Olivos, D.R., Alhadeff, A.L., Pierce, R.C., et al., 2013. The food intake-suppressive effects of glucagon-like peptide-1 receptor signaling in the ventral tegmental area are mediated by AMPA/kainate receptors. American Journal of Physiology Endocrinology and Metabolism 305:E1367-E1374.

[704] Ong, Z.Y., Liu, J.J., Pang, Z.P., Grill, H.J., 2017. Paraventricular thalamic control of food intake and reward: role of glucagon-like peptide- 1 receptor signaling. Neuropsychopharmacology 42:2387-2397.

[705] Terrill, S.J., Holt, M.K., Maske, C.B., Abrams, N., Reimann, F., Trapp, S., et al., 2019. Endogenous GLP-1 in lateral septum promotes satiety and suppresses motivation for food in mice. Physiology and Behavior 206:191199. 
[706] Hayes, M.R., Leichner, T.M., Zhao, S., Lee, G.S., Chowansky, A., Zimmer, D. et al., 2011. Intracellular signals mediating the food intake-suppressive effects of hindbrain glucagon-like peptide-1 receptor activation. Cell Metabolism 13:320-330.

[707] Minokoshi, Y., Alquier, T., Furukawa, N., Kim, Y.B., Lee, A., Xue, B., et al., 2004. AMP-kinase regulates food intake by responding to hormonal and nutrient signals in the hypothalamus. Nature 428:569-574.

[708] Fan, X., Ding, Y., Brown, S., Zhou, L., Shaw, M., Vella, M.C., et al., 2009. Hypothalamic AMP-activated protein kinase activation with AICAR amplifies counterregulatory responses to hypoglycemia in a rodent model of type 1 diabetes. American Journal of Physiology Regulatory Integrative and Comparative Physiology 296:R1702-R1708.

[709] McCrimmon, R.J., Fan, X., Ding, Y., Zhu, W., Jacob, R.J., Sherwin, R.S., 2004. Potential role for AMP-activated protein kinase in hypoglycemia sensing in the ventromedial hypothalamus. Diabetes 53:1953-1958.

[710] McCrimmon, R.J., Shaw, M., Fan, X., Cheng, H., Ding, Y., Vella, M.C., et al., 2008. Key role for AMP-activated protein kinase in the ventromedial hypothalamus in regulating counterregulatory hormone responses to acute hypoglycemia. Diabetes 57:444-450.

[711] Kohno, D., Sone, H., Tanaka, S., Kurita, H., Gantulga, D., Yada, T., 2011. AMP-activated protein kinase activates neuropeptide $Y$ neurons in the hypothalamic arcuate nucleus to increase food intake in rats. Neuroscience Letters 499:194-198.

[712] Burmeister, M.A., Ferre, T., Ayala, J.E., King, E.M., Holt, R.M., Ayala, J.E., 2012. Acute activation of central GLP-1 receptors enhances hepatic insulin action and insulin secretion in high-fat-fed, insulin resistant mice. American Journal of Physiology Endocrinology and Metabolism 302:E334-E343.

[713] Seo, S., Ju, S., Chung, H., Lee, D., Park, S., 2008. Acute effects of glucagonlike peptide-1 on hypothalamic neuropeptide and AMP activated kinase expression in fasted rats. Endocrine Journal 55:867-874.

[714] Burmeister, M.A., Ayala, J., Drucker, D.J., Ayala, J.E., 2013. Central glucagon-like peptide 1 receptor-induced anorexia requires glucose metabolism-mediated suppression of AMPK and is impaired by central fructose. American Journal of Physiology Endocrinology and Metabolism 304: E677-E685.

[715] Burmeister, M.A., Brown, J.D., Ayala, J.E., Stoffers, D.A., Sandoval, D.A., Seeley, R.J., et al., 2017. The glucagon-like peptide-1 receptor in the ventromedial hypothalamus reduces short-term food intake in male mice by regulating nutrient sensor activity. American Journal of Physiology Endocrinology and Metabolism 313:E651-E662.

[716] Alhadeff, A.L., Grill, H.J., 2014. Hindbrain nucleus tractus solitarius glucagonlike peptide-1 receptor signaling reduces appetitive and motivational aspects of feeding. American Journal of Physiology Regulatory Integrative and Comparative Physiology 307:R465-R470.

[717] Hayes, M.R., Bradley, L., Grill, H.J., 2009. Endogenous hindbrain glucagonlike peptide-1 receptor activation contributes to the control of food intake by mediating gastric satiation signaling. Endocrinology 150:2654-2659.

[718] Adams, J.M., Pei, H., Sandoval, D.A., Seeley, R.J., Chang, R.B., Liberles, S.D., et al., 2018. Liraglutide modulates appetite and body weight through glucagon-like peptide 1 receptor-expressing glutamatergic neurons. Diabetes 67:1538-1548.

[719] Chaudhri, O.B., Parkinson, J.R., Kuo, Y.T., Druce, M.R., Herlihy, A.H., Bell, J.D., et al., 2006. Differential hypothalamic neuronal activation following peripheral injection of GLP-1 and oxyntomodulin in mice detected by manganese-enhanced magnetic resonance imaging. Biochemical and Biophysical Research Communications 350:298-306.

[720] Parkinson, J.R., Chaudhri, O.B., Kuo, Y.T., Field, B.C., Herlihy, A.H., Dhillo, W.S., et al., 2009. Differential patterns of neuronal activation in the brainstem and hypothalamus following peripheral injection of GLP-1, oxyntomodulin and lithium chloride in mice detected by manganese-enhanced magnetic resonance imaging (MEMRI). Neuroimage 44:1022-1031.
[721] Schick, R.R., Zimmermann, J.P., vorm Walde, T., Schusdziarra, V., 2003. Peptides that regulate food intake: glucagon-like peptide 1-(7-36) amide acts at lateral and medial hypothalamic sites to suppress feeding in rats. American Journal of Physiology Regulatory Integrative and Comparative Physiology 284:R1427-R1435.

[722] McMahon, L.R., Wellman, P.J., 1997. Decreased intake of a liquid diet in nonfood-deprived rats following intra-PVN injections of GLP-1 (7-36) amide. Pharmacology Biochemistry and Behavior 58:673-677.

[723] McMahon, L.R., Wellman, P.J., 1998. PVN infusion of GLP-1-(7-36) amide suppresses feeding but does not induce aversion or alter locomotion in rats. American Journal of Physiology 274:R23-R29.

[724] Curtis, K.S., Sved, A.F., Verbalis, J.G., Stricker, E.M., 1994. Lithium chlorideinduced anorexia, but not conditioned taste aversions, in rats with area postrema lesions. Brain Research 663:30-37.

[725] Rabin, B.M., Hunt, W.A., 1992. Relationship between vomiting and taste aversion learning in the ferret: studies with ionizing radiation, lithium chloride, and amphetamine. Behavioral and Neural Biology 58:83-93.

[726] Thiele, T.E., Roitman, M.F., Bernstein, I.L., 1996. c-Fos induction in rat brainstem in response to ethanol- and lithium chloride-induced conditioned taste aversions. Alcoholism Clinical and Experimental Research 20:10231028.

[727] Mitchell, D., Wells, C., Hoch, N., Lind, K., Woods, S.C., Mitchell, L.K., 1976. Poison induced pica in rats. Physiology and Behavior 17:691-697.

[728] Pelchat, M.L., Grill, H.J., Rozin, P., Jacobs, J., 1983. Quality of acquired responses to tastes by Rattus norvegicus depends on type of associated discomfort. Journal of Comparative Psychology 97:140-153.

[729] Swank, M.W., Bernstein, I.L., 1994. c-Fos induction in response to a conditioned stimulus after single trial taste aversion learning. Brain Research 636:202-208.

[730] Thiele, T.E., Seeley, R.J., D’Alessio, D., Eng, J., Bernstein, I.L., Woods, S.C., et al., 1998. Central infusion of glucagon-like peptide-1-(7-36) amide (GLP-1) receptor antagonist attenuates lithium chloride-induced c-Fos induction in rat brainstem. Brain Research 801:164-170.

[731] Meachum, C.L., Bernstein, I.L., 1992. Behavioral conditioned responses to contextual and odor stimuli paired with LiCl administration. Physiology and Behavior 52:895-899.

[732] Bernstein, I.L., Chavez, M., Allen, D., Taylor, E.M., 1992. Area postrema mediation of physiological and behavioral effects of lithium chloride in the rat. Brain Research 575:132-137.

[733] O'Shea, D., Gunn, I., Chen, X., Bloom, S., Herbert, J., 1996. A role for central glucagon-like peptide-1 in temperature regulation. Neuroreport 7: $830-832$.

[734] Bettge, K., Kahle, M., Abd El Aziz, M.S., Meier, J.J., Nauck, M.A., 2017. Occurrence of nausea, vomiting and diarrhoea reported as adverse events in clinical trials studying glucagon-like peptide-1 receptor agonists: A systematic analysis of published clinical trials. Diabetes Obesity and Metabolism 19:336-347.

[735] Shiomi, M., Takada, T., Tanaka, Y., Yajima, K., Isomoto, A., Sakamoto, M., et al., 2018. Clinical factors associated with the occurrence of nausea and vomiting in type 2 diabetes patients treated with glucagon-like peptide-1 receptor agonists. Journal of Diabetes Investigation.

[736] Thiele, T.E., Van Dijk, G., Campfield, L.A., Smith, F.J., Burn, P., Woods, S.C., et al., 1997. Central infusion of GLP-1, but not leptin, produces conditioned taste aversions in rats. American Journal of Physiology 272:R726-R730.

[737] van Dijk, G., Thiele, T.E., Seeley, R.J., Woods, S.C., Bernstein, I.L., 1997. Glucagon-like peptide-1 and satiety. Nature 385:214.

[738] Borner, T., Liberini, C.G., Lutz, T.A., Riediger, T., 2018. Brainstem GLP-1 signalling contributes to cancer anorexia-cachexia syndrome in the rat. Neuropharmacology 131:282-290.

[739] Grill, H.J., Carmody, J.S., Amanda Sadacca, L., Williams, D.L., Kaplan, J.M., 2004. Attenuation of lipopolysaccharide anorexia by antagonism of caudal 
brain stem but not forebrain GLP-1-R. American Journal of Physiology Regulatory Integrative and Comparative Physiology 287:R1190-R1193.

[740] Alhadeff, A.L., Baird, J.P., Swick, J.C., Hayes, M.R., Grill, H.J., 2014. Glucagon-like Peptide-1 receptor signaling in the lateral parabrachial nucleus contributes to the control of food intake and motivation to feed. Neuropsychopharmacology 39:2233-2243.

[741] Alhadeff, A.L., Rupprecht, L.E., Hayes, M.R., 2012. GLP-1 neurons in the nucleus of the solitary tract project directly to the ventral tegmental area and nucleus accumbens to control for food intake. Endocrinology 153:647-658.

[742] Jelsing, J., Vrang, N., Hansen, G., Raun, K., Tang-Christensen, M., Knudsen, L.B., 2012. Liraglutide: short-lived effect on gastric emptying - long lasting effects on body weight. Diabetes Obesity and Metabolism 14:531538.

[743] ten Kulve, J.S., Veltman, D.J., van Bloemendaal, L., Barkhof, F., Deacon, C.F., Holst, J.J., et al., 2015. Endogenous GLP-1 mediates postprandial reductions in activation in central reward and satiety areas in patients with type 2 diabetes. Diabetologia 58:2688-2698.

[744] Dossat, A.M., Lilly, N., Kay, K., Williams, D.L., 2011. Glucagon-like peptide 1 receptors in nucleus accumbens affect food intake. Journal of Neuroscience 31:14453-14457.

[745] Terrill, S.J., Jackson, C.M., Greene, H.E., Lilly, N., Maske, C.B., Vallejo, S., et al., 2016. Role of lateral septum glucagon-like peptide 1 receptors in food intake. American Journal of Physiology Regulatory Integrative and Comparative Physiology 311:R124-R132.

[746] Decarie-Spain, L., Fisette, A., Zhu, Z., Yang, B., DiMarchi, R.D., Tschop, M.H., et al., 2019. GLP-1/dexamethasone inhibits food reward without inducing mood and memory deficits in mice. Neuropharmacology 151:55-63.

[747] Egecioglu, E., Steensland, P., Fredriksson, I., Feltmann, K., Engel, J.A., Jerlhag, E., 2013. The glucagon-like peptide 1 analogue Exendin-4 attenuates alcohol mediated behaviors in rodents. Psychoneuroendocrinology 38 : $1259-1270$.

[748] Sirohi, S., Schurdak, J.D., Seeley, R.J., Benoit, S.C., Davis, J.F., 2016. Central \& peripheral glucagon-like peptide-1 receptor signaling differentially regulate addictive behaviors. Physiology and Behavior 161:140-144.

[749] Dossat, A.M., Diaz, R., Gallo, L., Panagos, A., Kay, K., Williams, D.L., 2013. Nucleus accumbens GLP-1 receptors influence meal size and palatability. American Journal of Physiology Endocrinology and Metabolism 304:E1314E1320.

[750] Hunter, K., Holscher, C., 2012. Drugs developed to treat diabetes, liraglutide and lixisenatide, cross the blood brain barrier and enhance neurogenesis. BMC Neuroscience 13:33.

[751] Kastin, A.J., Akerstrom, V., 2003. Entry of exendin-4 into brain is rapid but may be limited at high doses. International Journal of Obesity and Related Metabolic Disorders 27:313-318.

[752] Kastin, A.J., Akerstrom, V., Pan, W., 2002. Interactions of glucagon-like peptide-1 (GLP-1) with the blood-brain barrier. Journal of Molecular Neuroscience 18:7-14.

[753] Salinas, C.B.G., Lu, T.T., Gabery, S., Marstal, K., Alanentalo, T., Mercer, A.J., et al., 2018. Integrated Brain Atlas for Unbiased Mapping of Nervous System Effects Following Liraglutide Treatment. Scientific Reports 8:10310.

[754] Orskov, C., Poulsen, S.S., Moller, M., Holst, J.J., 1996. Glucagon-like peptide I receptors in the subfornical organ and the area postrema are accessible to circulating glucagon-like peptide I. Diabetes 45:832-835.

[755] Gutzwiller, J.P., Hruz, P., Huber, A.R., Hamel, C., Zehnder, C., Drewe, J., et al., 2006. Glucagon-like peptide-1 is involved in sodium and water homeostasis in humans. Digestion 73:142-150.

[756] McKay, N.J., Daniels, D., 2013. Glucagon-like peptide-1 receptor agonist administration suppresses both water and saline intake in rats. Journal of Neuroendocrinology 25:929-938.
[757] McKay, N.J., Galante, D.L., Daniels, D., 2014. Endogenous glucagon-like peptide-1 reduces drinking behavior and is differentially engaged by water and food intakes in rats. Journal of Neuroscience 34:16417-16423.

[758] McKay, N.J., Kanoski, S.E., Hayes, M.R., Daniels, D., 2011. Glucagon-like peptide-1 receptor agonists suppress water intake independent of effects on food intake. American Journal of Physiology Regulatory Integrative and Comparative Physiology 301:R1755-R1764.

[759] Navarro, M., Rodriquez de Fonseca, F., Alvarez, E., Chowen, J.A., Zueco, J.A. Gomez, R., et al., 1996. Colocalization of glucagon-like peptide-1 (GLP-1) receptors, glucose transporter GLUT-2, and glucokinase mRNAs in rat hypothalamic cells: evidence for a role of GLP-1 receptor agonists as an inhibitory signal for food and water intake. Journal of Neurochemistry 67: 1982-1991.

[760] Tang-Christensen, M., Vrang, N., Larsen, P.J., 1998. Glucagon-like peptide 1(7-36) amide's central inhibition of feeding and peripheral inhibition of drinking are abolished by neonatal monosodium glutamate treatment. Diabetes 47:530-537.

[761] Moreno, C., Mistry, M., Roman, R.J., 2002. Renal effects of glucagon-like peptide in rats. European Journal of Pharmacology 434:163-167.

[762] Muskiet, M.H., Tonneijck, L., Smits, M.M., Kramer, M.H., Diamant, M., Joles, J.A., et al., 2016. Acute renal haemodynamic effects of glucagon-like peptide-1 receptor agonist exenatide in healthy overweight men. Diabetes Obesity and Metabolism 18:178-185.

[763] Skov, J., Pedersen, M., Holst, J.J., Madsen, B., Goetze, J.P., Rittig, S., et al., 2016. Short-term effects of liraglutide on kidney function and vasoactive hormones in type 2 diabetes: a randomized clinical trial. Diabetes Obesity and Metabolism 18:581-589.

[764] Tonneijck, L., Smits, M.M., Muskiet, M.H.A., Hoekstra, T., Kramer, M.H.H., Danser, A.H.J., et al., 2016. Acute renal effects of the GLP-1 receptor agonist exenatide in overweight type 2 diabetes patients: a randomised, doubleblind, placebo-controlled trial. Diabetologia 59:1412-1421.

[765] Tonneijck, L., Smits, M.M., Muskiet, M.H., Hoekstra, T., Kramer, M.H., Danser, A.H., et al., 2016. Renal Effects of DPP-4 Inhibitor Sitagliptin or GLP1 Receptor Agonist Liraglutide in Overweight Patients With Type 2 Diabetes: A 12-Week, Randomized, Double-Blind, Placebo-Controlled Trial. Diabetes Care 39:2042-2050.

[766] Fujita, H., Morii, T., Fujishima, H., Sato, T., Shimizu, T., Hosoba, M., et al., 2014. The protective roles of GLP-1R signaling in diabetic nephropathy: possible mechanism and therapeutic potential. Kidney International 85:579_589.

[767] Korner, M., Stockli, M., Waser, B., Reubi, J.C., 2007. GLP-1 receptor expression in human tumors and human normal tissues: potential for in vivo targeting. Journal of Nuclear Medicine 48:736-743.

[768] Schlatter, P., Beglinger, C., Drewe, J., Gutmann, H., 2007. Glucagon-like peptide 1 receptor expression in primary porcine proximal tubular cells. Regulatory Peptides 141:120-128.

[769] Pacheco, B.P., Crajoinas, R.O., Couto, G.K., Davel, A.P., Lessa, L.M., Rossoni, L.V., et al., 2011. Dipeptidyl peptidase IV inhibition attenuates blood pressure rising in young spontaneously hypertensive rats. Journal of $\mathrm{Hy}-$ pertension 29:520-528.

[770] Thomson, S.C., Kashkouli, A., Singh, P., 2013. Glucagon-like peptide-1 receptor stimulation increases GFR and suppresses proximal reabsorption in the rat. American Journal of Physiology Renal Physiology 304:F137-F144.

[771] Carraro-Lacroix, L.R., Malnic, G., Girardi, A.C., 2009. Regulation of $\mathrm{Na}+/ \mathrm{H}+$ exchanger NHE3 by glucagon-like peptide 1 receptor agonist exendin-4 in renal proximal tubule cells. American Journal of Physiology Renal Physiology 297:F1647-F1655.

[772] Farah, L.X., Valentini, V., Pessoa, T.D., Malnic, G., McDonough, A.A., Girardi, A.C., 2016. The physiological role of glucagon-like peptide-1 in the 
regulation of renal function. American Journal of Physiology Renal Physiology 310:F123-F127.

[773] Lockie, S.H., Heppner, K.M., Chaudhary, N., Chabenne, J.R., Morgan, D.A., Veyrat-Durebex, C., et al., 2012. Direct control of brown adipose tissue thermogenesis by central nervous system glucagon-like peptide-1 receptor signaling. Diabetes 61:2753-2762.

[774] Heppner, K.M., Marks, S., Holland, J., Ottaway, N., Smiley, D., Dimarchi, R., et al., 2015. Contribution of brown adipose tissue activity to the control of energy balance by GLP-1 receptor signalling in mice. Diabetologia 58:21242132.

[775] Cannon, B., Nedergaard, J., 2011. Nonshivering thermogenesis and its adequate measurement in metabolic studies. Journal of Experimental Biology 214:242-253.

[776] Morrison, S.F., Madden, C.J., Tupone, D., 2014. Central neural regulation of brown adipose tissue thermogenesis and energy expenditure. Cell Metabolism 19:741-756.

[777] Lee, S.J., Sanchez-Watts, G., Krieger, J.P., Pignalosa, A., Norell, P.N., Cortella, A., et al., 2018. Loss of dorsomedial hypothalamic GLP-1 signaling reduces BAT thermogenesis and increases adiposity. Molecular Metabolism 11:33-46.

[778] Shi, X., Chacko, S., Li, F., Li, D., Burrin, D., Chan, L., et al., 2017. Acute activation of GLP-1-expressing neurons promotes glucose homeostasis and insulin sensitivity. Molecular Metabolism 6:1350-1359.

[779] Hwa, J.J., Ghibaudi, L., Williams, P., Witten, M.B., Tedesco, R., Strader, C.D., 1998. Differential effects of intracerebroventricular glucagon-like peptide-1 on feeding and energy expenditure regulation. Peptides 19:869-875.

[780] Osaka, T., Endo, M., Yamakawa, M., Inoue, S., 2005. Energy expenditure by intravenous administration of glucagon-like peptide- 1 mediated by the lower brainstem and sympathoadrenal system. Peptides 26:1623-1631.

[781] Krieger, J.P., Santos da Conceicao, E.P., Sanchez-Watts, G., Arnold, M., Pettersen, K.G., Mohammed, M., et al., 2018. Glucagon-like peptide-1 regulates brown adipose tissue thermogenesis via the gut-brain axis in rats. American Journal of Physiology Regulatory Integrative and Comparative Physiology 315:R708-R720.

[782] Skibicka, K.P., Alhadeff, A.L., Grill, H.J., 2009. Hindbrain cocaine- and amphetamine-regulated transcript induces hypothermia mediated by GLP-1 receptors. Journal of Neuroscience 29:6973-6981.

[783] Cegla, J., Troke, R.C., Jones, B., Tharakan, G., Kenkre, J., McCullough, K.A., et al., 2014. Coinfusion of low-dose GLP-1 and glucagon in man results in a reduction in food intake. Diabetes 63:3711-3720.

[784] Tan, T., Behary, P., Tharakan, G., Minnion, J., Al-Najim, W., Albrechtsen, N.J.W., et al., 2017. The Effect of a Subcutaneous Infusion of GLP-1, OXM, and PYY on Energy Intake and Expenditure in Obese Volunteers. The Journal of Cinical Endocrinology and Metabolism 102:2364-2372.

[785] Tan, T.M., Field, B.C., McCullough, K.A., Troke, R.C., Chambers, E.S., Salem, V., et al., 2013. Coadministration of glucagon-like peptide-1 during glucagon infusion in humans results in increased energy expenditure and amelioration of hyperglycemia. Diabetes 62:1131-1138.

[786] Bagger, J.I., Holst, J.J., Hartmann, B., Andersen, B., Knop, F.K., Vilsboll, T., 2015. Effect of Oxyntomodulin, Glucagon, GLP-1, and Combined Glucagon + GLP-1 Infusion on Food Intake, Appetite, and Resting Energy Expenditure. The Journal of Cinical Endocrinology and Metabolism 100: $4541-4552$

[787] Bergmann, N.C., Lund, A., Gasbjerg, L.S., Meessen, E.C.E., Andersen, M.M., Bergmann, S., et al., 2019. Effects of combined GIP and GLP-1 infusion on energy intake, appetite and energy expenditure in overweight/obese individuals: a randomised, crossover study. Diabetologia 62:665-675.

[788] Flint, A., Raben, A., Rehfeld, J.F., Holst, J.J., Astrup, A., 2000. The effect of glucagon-like peptide-1 on energy expenditure and substrate metabolism in humans. International Journal of Obesity and Related Metabolic Disorders 24: 288-298.
[789] Linnebjerg, H., Park, S., Kothare, P.A., Trautmann, M.E., Mace, K., Fineman, M., et al., 2008. Effect of exenatide on gastric emptying and relationship to postprandial glycemia in type 2 diabetes. Regulatory Peptides 151:123-129.

[790] Little, T.J., Pilichiewicz, A.N., Russo, A., Phillips, L., Jones, K.L., Nauck, M.A., et al., 2006. Effects of intravenous glucagon-like peptide-1 on gastric emptying and intragastric distribution in healthy subjects: relationships with postprandial glycemic and insulinemic responses. The Journal of Cinical Endocrinology and Metabolism 91:1916-1923.

[791] Wettergren, A., Schjoldager, B., Mortensen, P.E., Myhre, J., Christiansen, J., Holst, J.J., 1993. Truncated GLP-1 (proglucagon 78-107-amide) inhibits gastric and pancreatic functions in man. Digestive Diseases and Sciences 38: 665-673.

[792] Tatarkiewicz, K., Sablan, E.J., Polizzi, C.J., Villescaz, C., Parkes, D.G., 2014. Long-term metabolic benefits of exenatide in mice are mediated solely via the known glucagon-like peptide 1 receptor. American Journal of Physiology Regulatory Integrative and Comparative Physiology 306:R490-R498.

[793] Bozkurt, A., Naslund, E., Holst, J.J., Hellstrom, P.M., 2002. GLP-1 and GLP-2 act in concert to inhibit fasted, but not fed, small bowel motility in the rat. Regulatory Peptides 107:129-135.

[794] Giralt, M., Vergara, P., 1998. Sympathetic pathways mediate GLP-1 actions in the gastrointestinal tract of the rat. Regulatory Peptides 74:19-25.

[795] Imeryuz, N., Yegen, B.C., Bozkurt, A., Coskun, T., VillanuevaPenacarrillo, M.L., Ulusoy, N.B., 1997. Glucagon-like peptide-1 inhibits gastric emptying via vagal afferent-mediated central mechanisms. American Journal of Physiology 273:G920-G927.

[796] Anvari, M., Paterson, C.A., Daniel, E.E., McDonald, T.J., 1998. Effects of GLP1 on gastric emptying, antropyloric motility, and transpyloric flow in response to a nonnutrient liquid. Digestive Diseases and Sciences 43:1133-1140.

[797] Ionut, V., Zheng, D., Stefanovski, D., Bergman, R.N., 2008. Exenatide can reduce glucose independent of islet hormones or gastric emptying. American Journal of Physiology Endocrinology and Metabolism 295:E269-E277.

[798] Moore, M.C., Werner, U., Smith, M.S., Farmer, T.D., Cherrington, A.D., 2013. Effect of the glucagon-like peptide-1 receptor agonist lixisenatide on postprandial hepatic glucose metabolism in the conscious dog. American Journal of Physiology Endocrinology and Metabolism 305:E1473-E1482.

[799] Nagell, C.F., Wettergren, A., Orskov, C., Holst, J.J., 2006. Inhibitory effect of GLP-1 on gastric motility persists after vagal deafferentation in pigs. Scandinavian Journal of Gastroenterology 41:667-672.

[800] Wettergren, A., Wojdemann, M., Holst, J.J., 1998. Glucagon-like peptide-1 inhibits gastropancreatic function by inhibiting central parasympathetic outflow. American Journal of Physiology 275:G984-G992.

[801] Halim, M.A., Degerblad, M., Sundbom, M., Karlbom, U., Holst, J.J., Webb, D.L., et al., 2018. Glucagon-Like Peptide-1 Inhibits Prandial Gastrointestinal Motility Through Myenteric Neuronal Mechanisms in Humans. The Journal of Cinical Endocrinology and Metabolism 103:575-585.

[802] Nakatani, Y., Maeda, M., Matsumura, M., Shimizu, R., Banba, N., Aso, Y., et al., 2017. Effect of GLP-1 receptor agonist on gastrointestinal tract motility and residue rates as evaluated by capsule endoscopy. Diabetes and Metabolism 43:430-437.

[803] Nauck, M.A., Kemmeries, G., Holst, J.J., Meier, J.J., 2011. Rapid tachyphylaxis of the glucagon-like peptide 1-induced deceleration of gastric emptying in humans. Diabetes 60:1561-1565.

[804] Nauck, M.A., Wollschlager, D., Werner, J., Holst, J.J., Orskov, C., Creutzfeldt, W., et al., 1996. Effects of subcutaneous glucagon-like peptide 1 (GLP-1 [7-36 amide]) in patients with NIDDM. Diabetologia 39:1546-1553.

[805] Thazhath, S.S., Marathe, C.S., Wu, T., Chang, J., Khoo, J., Kuo, P., et al., 2016. The Glucagon-like peptide 1 receptor agonist exenatide inhibits small intestinal motility, flow, transit, and absorption of glucose in healthy subjects and patients with type 2 diabetes: a randomized controlled trial. Diabetes 65 : $269-275$. 
[806] Hayes, M.R., Skibicka, K.P., Grill, H.J., 2008. Caudal brainstem processing is sufficient for behavioral, sympathetic, and parasympathetic responses driven by peripheral and hindbrain glucagon-like-peptide-1 receptor stimulation. Endocrinology 149:4059-4068.

[807] Wettergren, A., Wojdemann, M., Meisner, S., Stadil, F., Holst, J.J., 1997. The inhibitory effect of glucagon-like peptide-1 (GLP-1) 7-36 amide on gastric acid secretion in humans depends on an intact vagal innervation. Gut 40 : 597-601.

[808] Halawi, H., Khemani, D., Eckert, D., O'Neill, J., Kadouh, H., Grothe, K., et al., 2017. Effects of liraglutide on weight, satiation, and gastric functions in obesity: a randomised, placebo-controlled pilot trial. Lancet Gastroenterology and Hepatology 2:890-899.

[809] Nauck, M.A., Niedereichholz, U., Ettler, R., Holst, J.J., Orskov, C., Ritzel, R., et al., 1997. Glucagon-like peptide 1 inhibition of gastric emptying outweighs its insulinotropic effects in healthy humans. American Journal of Physiology 273:E981-E988.

[810] Nauck, M.A., 1999. Is glucagon-like peptide 1 an incretin hormone? Diabetologia 42:373-379.

[811] Herman, J.P., 2018. Regulation of hypothalamo-pituitary-adrenocortical responses to stressors by the nucleus of the solitary tract/dorsal vagal complex. Cellular and Molecular Neurobiology 38:25-35.

[812] Sarkar, S., Fekete, C., Legradi, G., Lechan, R.M., 2003. Glucagon like peptide-1 (7-36) amide (GLP-1) nerve terminals densely innervate corticotropin-releasing hormone neurons in the hypothalamic paraventricular nucleus. Brain Research 985:163-168.

[813] Ghosal, S., Packard, A.E.B., Mahbod, P., McKlveen, J.M., Seeley, R.J., Myers, B., et al., 2017. Disruption of glucagon-like peptide 1 signaling in Sim1 neurons reduces physiological and behavioral reactivity to acute and chronic stress. Journal of Neuroscience 37:184-193.

[814] Rowland, N.E., Crews, E.C., Gentry, R.M., 1997. Comparison of Fos induced in rat brain by GLP-1 and amylin. Regulatory Peptides 71:171-174.

[815] Gil-Lozano, M., Perez-Tilve, D., Alvarez-Crespo, M., Martis, A., Fernandez, A.M., Catalina, P.A., et al., 2010. GLP-1(7-36)-amide and Exendin-4 stimulate the HPA axis in rodents and humans. Endocrinology 151: $2629-2640$

[816] Malendowicz, L.K., Neri, G., Nussdorfer, G.G., Nowak, K.W., Zyterska, A., Ziolkowska, A., 2003. Prolonged exendin-4 administration stimulates pituitary-adrenocortical axis of normal and streptozotocin-induced diabetic rats. International Journal of Molecular Medicine 12:593-596.

[817] Malendowicz, L.K., Nussdorfer, G.G., Nowak, K.W., Ziolkowska, A., Tortorella, C., Trejter, M., 2003. Exendin-4, a GLP-1 receptor agonist, stimulates pituitary-adrenocortical axis in the rat: Investigations into the mechanism(s) underlying Ex4 effect. International Journal of Molecular Medicine 12:237-241.

[818] Krass, M., Volke, A., Runkorg, K., Wegener, G., Lund, S., Abildgaard, A., et al., 2015. GLP-1 receptor agonists have a sustained stimulatory effect on corticosterone release after chronic treatment. Acta Neuropsychiatrica 27:25-32.

[819] Lee, S.J., Diener, K., Kaufman, S., Krieger, J.P., Pettersen, K.G., Jejelava, N., et al., 2016. Limiting glucocorticoid secretion increases the anorexigenic property of Exendin-4. Molecular Metabolism 5:552-565.

[820] Quarta, C., Clemmensen, C., Zhu, Z., Yang, B., Joseph, S.S., Lutter, D., et al., 2017. Molecular integration of incretin and glucocorticoid action reverses immunometabolic dysfunction and obesity. Cell Metabolism 26:620-632 e626.

[821] Alvarez, E., Roncero, I., Chowen, J.A., Thorens, B., Blazquez, E., 1996. Expression of the glucagon-like peptide-1 receptor gene in rat brain. Journal of Neurochemistry 66:920-927.

[822] Rebosio, C., Balbi, M., Passalacqua, M., Ricciarelli, R., Fedele, E., 2018. Presynaptic GLP-1 receptors enhance the depolarization-evoked release of glutamate and GABA in the mouse cortex and hippocampus. Biofactors 44: $148-157$.
[823] Kandel, E.R., 2001. The molecular biology of memory storage: a dialogue between genes and synapses. Science 294:1030-1038.

[824] Lamsa, K., Lau, P., 2018. Long-term plasticity of hippocampal interneurons during in vivo memory processes. Current Opinion in Neurobiology 54:2027.

[825] Perry, T., Lahiri, D.K., Chen, D., Zhou, J., Shaw, K.T., Egan, J.M., et al., 2002. A novel neurotrophic property of glucagon-like peptide 1: a promoter of nerve growth factor-mediated differentiation in PC12 cells. Journal of Pharmacology and Experimental Therapeutics 300:958-966.

[826] Perry, T., Haughey, N.J., Mattson, M.P., Egan, J.M., Greig, N.H., 2002. Protection and reversal of excitotoxic neuronal damage by glucagon-like peptide- 1 and exendin-4. Journal of Pharmacology and Experimental Therapeutics 302:881-888.

[827] Perry, T., Greig, N.H., 2002. The glucagon-like peptides: a new genre in therapeutic targets for intervention in Alzheimer's disease. Journal of Alzheimer's Disease 4:487-496.

[828] Warby, S.C., Montpetit, A., Hayden, A.R., Carroll, J.B., Butland, S.L., Visscher, H., et al., 2009. CAG expansion in the Huntington disease gene is associated with a specific and targetable predisposing haplogroup. The American Journal of Human Genetics 84:351-366.

[829] Vidoni, C., Castiglioni, A., Seca, C., Secomandi, E., Melone, M.A., Isidoro, C. 2016. Dopamine exacerbates mutant Huntingtin toxicity via oxidativemediated inhibition of autophagy in SH-SY5Y neuroblastoma cells: Beneficial effects of anti-oxidant therapeutics. Neurochemistry International 101: $132-143$.

[830] Lalic, N.M., Maric, J., Svetel, M., Jotic, A., Stefanova, E., Lalic, K., et al., 2008. Glucose homeostasis in Huntington disease: abnormalities in insulin sensitivity and early-phase insulin secretion. Archives of Neurology 65:476480.

[831] Chang, C.C., Lin, T.C., Ho, H.L., Kuo, C.Y., Li, H.H., Korolenko, T.A., et al., 2018. GLP-1 analogue liraglutide attenuates mutant huntingtin-induced neurotoxicity by restoration of neuronal insulin signaling. International Journal of Molecular Sciences 19.

[832] Qi, L., Ke, L., Liu, X., Liao, L., Ke, S., Liu, X., et al., 2016. Subcutaneous administration of liraglutide ameliorates learning and memory impairment by modulating tau hyperphosphorylation via the glycogen synthase kinase-3beta pathway in an amyloid beta protein induced alzheimer disease mouse model. European Journal of Pharmacology 783:23-32.

[833] Wang, X.H., Li, L., Holscher, C., Pan, Y.F., Chen, X.R., Qi, J.S., 2010. Val8glucagon-like peptide-1 protects against Abeta1-40-induced impairment of hippocampal late-phase long-term potentiation and spatial learning in rats. Neuroscience 170:1239-1248.

[834] Chen, S., Sun, J., Zhao, G., Guo, A., Chen, Y., Fu, R., et al., 2017. Liraglutide improves water maze learning and memory performance while reduces hyperphosphorylation of tau and neurofilaments in APP/PS1/Tau triple transgenic mice. Neurochemical Research 42:2326-2335.

[835] Hansen, H.H., Fabricius, K., Barkholt, P., Niehoff, M.L., Morley, J.E., Jelsing, J., et al., 2015. The GLP-1 receptor agonist liraglutide improves memory function and increases hippocampal CA1 neuronal numbers in a senescence-accelerated mouse model of Alzheimer's disease. Journal of Alzheimer's Disease 46:877-888.

[836] McClean, P.L., Parthsarathy, V., Faivre, E., Holscher, C., 2011. The diabetes drug liraglutide prevents degenerative processes in a mouse model of Alzheimer's disease. Journal of Neuroscience 31:6587-6594.

[837] Gejl, M., Brock, B., Egefjord, L., Vang, K., Rungby, J., Gjedde, A., 2017. Blood-brain glucose transfer in Alzheimer's disease: Effect of GLP-1 analog treatment. Scientific Reports 7:17490.

[838] Watson, K.T., Wroolie, T.E., Tong, G., Foland-Ross, L.C., Frangou, S., Singh, M., et al., 2019. Neural correlates of liraglutide effects in persons at risk for Alzheimer's disease. Behavioural Brain Research 356:271278. 
[839] Femminella, G.D., Frangou, E., Love, S.B., Busza, G., Holmes, C., Ritchie, C., et al., 2019. Evaluating the effects of the novel GLP-1 analogue liraglutide in Alzheimer's disease: study protocol for a randomised controlled trial (ELAD study). Trials 20:191.

[840] Burns, R.S., Chiueh, C.C., Markey, S.P., Ebert, M.H., Jacobowitz, D.M., Kopin, I.J., 1983. A primate model of parkinsonism: selective destruction of dopaminergic neurons in the pars compacta of the substantia nigra by $\mathrm{N}$ methyl-4-phenyl-1,2,3,6-tetrahydropyridine. Proceedings of the National Academy of Sciences of the United States of America 80:4546-4550.

[841] Chiueh, C.C., Markey, S.P., Burns, R.S., Johannessen, J.N., Jacobowitz, D.M., Kopin, I.J., 1984. Neurochemical and behavioral effects of 1-methyl-4-phenyl-1,2,3,6- tetrahydropyridine (MPTP) in rat, guinea pig, and monkey. Psychopharmacology Bulletin 20:548-553.

[842] Heikkila, R.E., Hess, A., Duvoisin, R.C., 1985. Dopaminergic neurotoxicity of 1-methyl-4-phenyl-1,2,5,6-tetrahydropyridine (MPTP) in the mouse: relationships between monoamine oxidase, MPTP metabolism and neurotoxicity. Life Sciences 36:231-236.

[843] Li, Y., Perry, T., Kindy, M.S., Harvey, B.K., Tweedie, D., Holloway, H.W., et al., 2009. GLP-1 receptor stimulation preserves primary cortical and dopaminergic neurons in cellular and rodent models of stroke and Parkinsonism. Proceedings of the National Academy of Sciences of the United States of America 106:1285-1290.

[844] Abuirmeileh, A., Harkavyi, A., Rampersaud, N., Lever, R., Tadross, J.A., Bloom, S.R., et al., 2012. Exendin-4 treatment enhances L-DOPA evoked release of striatal dopamine and decreases dyskinetic movements in the 6hydoxydopamine lesioned rat. Journal of Pharmacy and Pharmacology 64: 637-643.

[845] Harkavyi, A., Abuirmeileh, A., Lever, R., Kingsbury, A.E., Biggs, C.S., Whitton, P.S., 2008. Glucagon-like peptide 1 receptor stimulation reverses key deficits in distinct rodent models of Parkinson's disease. Journal of Neuroinflammation 5:19.

[846] Kim, S., Moon, M., Park, S., 2009. Exendin-4 protects dopaminergic neurons by inhibition of microglial activation and matrix metalloproteinase-3 expression in an animal model of Parkinson's disease. Journal of Endocrinology 202:431-439.

[847] Liu, W., Jalewa, J., Sharma, M., Li, G., Li, L., Holscher, C., 2015. Neuroprotective effects of lixisenatide and liraglutide in the 1-methyl-4-phenyl1,2,3,6-tetrahydropyridine mouse model of Parkinson's disease. Neuroscience 303:42-50.

[848] Rampersaud, N., Harkavyi, A., Giordano, G., Lever, R., Whitton, J., Whitton, P., 2012. Exendin-4 reverts behavioural and neurochemical dysfunction in a pre-motor rodent model of Parkinson's disease with noradrenergic deficit. British Journal of Pharmacology 167:1467-1479.

[849] Rampersaud, N., Harkavyi, A., Giordano, G., Lever, R., Whitton, J., Whitton, P.S., 2012. Exendin-4 reverses biochemical and behavioral deficits in a pre-motor rodent model of Parkinson's disease with combined noradrenergic and serotonergic lesions. Neuropeptides 46:183-193.

[850] Athauda, D., Maclagan, K., Skene, S.S., Bajwa-Joseph, M., Letchford, D., Chowdhury, K., et al., 2017. Exenatide once weekly versus placebo in Parkinson's disease: a randomised, double-blind, placebo-controlled trial. Lancet 390:1664-1675.

[851] Aviles-Olmos, I., Dickson, J., Kefalopoulou, Z., Djamshidian, A., Ell, P., Soderlund, T., et al., 2013. Exenatide and the treatment of patients with Parkinson's disease. Journal of Clinical Investigation 123:2730-2736.

[852] Aviles-Olmos, I., Dickson, J., Kefalopoulou, Z., Djamshidian, A., Kahan, J., Ell, P., et al., 2014. Motor and cognitive advantages persist 12 months after exenatide exposure in Parkinson's disease. Journal of Parkinson's Disease 4: $337-344$.

[853] Foltynie, T., Aviles-OImos, I., 2014. Exenatide as a potential treatment for patients with Parkinson's disease: first steps into the clinic. Alzheimers Dementia 10:S38-S46.
[854] Yamada, C., Yamada, Y., Tsukiyama, K., Yamada, K., Udagawa, N., Takahashi, N., et al., 2008. The murine glucagon-like peptide-1 receptor is essential for control of bone resorption. Endocrinology 149:574-579.

[855] Hegedus, L., Moses, A.C., Zdravkovic, M., Le Thi, T., Daniels, G.H., 2011. GLP-1 and calcitonin concentration in humans: lack of evidence of calcitonin release from sequential screening in over 5000 subjects with type 2 diabetes or nondiabetic obese subjects treated with the human GLP-1 analog, liraglutide. The Journal of Cinical Endocrinology and Metabolism 96:853-860.

[856] Bjerre Knudsen, L., Madsen, L.W., Andersen, S., Almholt, K., de Boer, A.S., Drucker, D.J., et al., 2010. Glucagon-like Peptide-1 receptor agonists activate rodent thyroid $\mathrm{C}$-cells causing calcitonin release and $\mathrm{C}$-cell proliferation. Endocrinology 151:1473-1486.

[857] Mabilleau, G., Mieczkowska, A., Irwin, N., Flatt, P.R., Chappard, D., 2013. Optimal bone mechanical and material properties require a functional glucagon-like peptide-1 receptor. Journal of Endocrinology 219:59-68.

[858] Mieczkowska, A., Irwin, N., Flatt, P.R., Chappard, D., Mabilleau, G., 2013. Glucose-dependent insulinotropic polypeptide (GIP) receptor deletion leads to reduced bone strength and quality. Bone 56:337-342.

[859] Gaudin-Audrain, C., Irwin, N., Mansur, S., Flatt, P.R., Thorens, B., Basle, M., et al., 2013. Glucose-dependent insulinotropic polypeptide receptor deficiency leads to modifications of trabecular bone volume and quality in mice. Bone 53:221-230.

[860] Mieczkowska, A., Mansur, S., Bouvard, B., Flatt, P.R., Thorens, B., Irwin, N., et al., 2015. Double incretin receptor knock-out (DIRKO) mice present with alterations of trabecular and cortical micromorphology and bone strength. Osteoporosis International 26:209-218.

[861] Meng, J., Ma, X., Wang, N., Jia, M., Bi, L., Wang, Y., et al., 2016. Activation of GLP-1 Receptor Promotes Bone Marrow Stromal Cell Osteogenic Differentiation through beta-Catenin. Stem Cell Reports 6:633.

[862] Mansur, S.A., Mieczkowska, A., Bouvard, B., Flatt, P.R., Chappard, D., Irwin, N., et al., 2015. Stable Incretin Mimetics Counter Rapid Deterioration of Bone Quality in Type 1 Diabetes Mellitus. Journal of Cellular Physiology 230: 3009-3018.

[863] Pereira, M., Gohin, S., Roux, J.P., Fisher, A., Cleasby, M.E., Mabilleau, G., et al., 2017. Exenatide Improves Bone Quality in a Murine Model of Genetically Inherited Type 2 Diabetes Mellitus. Frontiers in Endocrinology (Lausanne) 8:327.

[864] Mansur, S.A., Mieczkowska, A., Flatt, P.R., Chappard, D., Irwin, N., Mabilleau, G., 2019. The GLP-1 receptor agonist exenatide ameliorates bone composition and tissue material properties in high fat fed diabetic mice. Frontiers in Endocrinology (Lausanne) 10:51.

[865] Mansur, S.A., Mieczkowska, A., Flatt, P.R., Bouvard, B., Chappard, D., Irwin, N., et al., 2016. A new stable GIP-Oxyntomodulin hybrid peptide improved bone strength both at the organ and tissue levels in geneticallyinherited type 2 diabetes mellitus. Bone 87:102-113.

[866] Mabilleau, G., Gobron, B., Mieczkowska, A., Perrot, R., Chappard, D., 2018. Efficacy of targeting bone-specific GIP receptor in ovariectomy-induced bone loss. Journal of Endocrinology.

[867] Mabilleau, G., Mieczkowska, A., Irwin, N., Simon, Y., Audran, M., Flatt, P.R., et al., 2014. Beneficial effects of a N-terminally modified GIP agonist on tissue-level bone material properties. Bone 63:61-68.

[868] Mansur, S.A., Mieczkowska, A., Flatt, P.R., Chappard, D., Irwin, N., Mabilleau, G., 2019. Sitagliptin Alters Bone Composition in High-Fat-Fed Mice. Calcified Tissue International 104:437-448.

[869] Henriksen, D.B., Alexandersen, P., Bjarnason, N.H., Vilsboll, T., Hartmann, B., Henriksen, E.E., et al., 2003. Role of gastrointestinal hormones in postprandial reduction of bone resorption. Journal of Bone and Mineral Research 18:2180-2189.

[870] Eriksson, R., Broberg, B.V., Ishoy, P.L., Bak, N., Andersen, U.B., Jorgensen, N.R., et al., 2018. Bone Status in Obese, Non-diabetic, Antipsychotic-Treated Patients, and Effects of the Glucagon-Like Peptide-1 
Receptor Agonist Exenatide on Bone Turnover Markers and Bone Mineral Density. Frontiers in Psychiatry 9:781.

[871] lepsen, E.W., Lundgren, J.R., Hartmann, B., Pedersen, O., Hansen, T., Jorgensen, N.R., et al., 2015. GLP-1 receptor agonist treatment increases bone formation and prevents bone loss in weight-reduced obese women. The Journal of Cinical Endocrinology and Metabolism 100:2909-2917.

[872] Mabilleau, G., Mieczkowska, A., Chappard, D., 2014. Use of glucagon-like peptide-1 receptor agonists and bone fractures: a meta-analysis of randomized clinical trials. Journal of Diabetes 6:260-266.

[873] Driessen, J.H., Henry, R.M., van Onzenoort, H.A., Lalmohamed, A., Burden, A.M., Prieto-Alhambra, D., et al., 2015. Bone fracture risk is not associated with the use of glucagon-like peptide-1 receptor agonists: a population-based cohort analysis. Calcified Tissue International 97:104-112.

[874] Cheng, L., Hu, Y., Li, Y.Y., Cao, X., Bai, N., Lu, T.T., et al., 2019. Glucagonlike peptide-1 receptor agonists and risk of bone fracture in patients with type 2 diabetes mellitus: a meta-analysis of randomized controlled trials. Diabetes Metabolism in Research Review, e3168.

[875] Banting, F.G., Best, C.H., Collip, J.B., Campbell, W.R., Fletcher, A.A., 1922. Pancreatic Extracts in the Treatment of Diabetes Mellitus. Canadian Medical Association Journal 12:141-146.

[876] Vilsboll, T., Agerso, H., Krarup, T., Holst, J.J., 2003. Similar elimination rates of glucagon-like peptide- 1 in obese type 2 diabetic patients and healthy subjects. The Journal of Cinical Endocrinology and Metabolism 88:220-224.

[877] Peters, K.R., 2013. Liraglutide for the treatment of type 2 diabetes: a clinical update. American Journal of Therapeutics 20:178-188.

[878] Muller, T.D., Clemmensen, C., Finan, B., DiMarchi, R.D., Tschop, M.H., 2018. Anti-Obesity Therapy: from Rainbow Pills to Polyagonists. Pharmacological Reviews 70:712-746.

[879] Eng, J., 1992. Exendin peptides. Mount Sinai Journal of Medicine A Journal of Translational and Personalized Medicine 59:147-149.

[880] Neidigh, J.W., Fesinmeyer, R.M., Prickett, K.S., Andersen, N.H., 2001. Exendin-4 and glucagon-like-peptide-1: NMR structural comparisons in the solution and micelle-associated states. Biochemistry 40:1318813200.

[881] Barnett, A.H., Burger, J., Johns, D., Brodows, R., Kendall, D.M., Roberts, A., et al., 2007. Tolerability and efficacy of exenatide and titrated insulin glargine in adult patients with type 2 diabetes previously uncontrolled with metformin or a sulfonylurea: a multinational, randomized, open-label, two-period, crossover noninferiority trial. Clinical Therapeutics 29:2333-2348.

[882] DeFronzo, R.A., Ratner, R.E., Han, J., Kim, D.D., Fineman, M.S., Baron, A.D., 2005. Effects of exenatide (exendin-4) on glycemic control and weight over 30 weeks in metformin-treated patients with type 2 diabetes. Diabetes Care 28:1092-1100.

[883] Kolterman, 0.G., Buse, J.B., Fineman, M.S., Gaines, E., Heintz, S., Bicsak, T.A., et al., 2003. Synthetic exendin-4 (exenatide) significantly reduces postprandial and fasting plasma glucose in subjects with type 2 diabetes. The Journal of Cinical Endocrinology and Metabolism 88:30823089.

[884] Poon, T., Nelson, P., Shen, L., Mihm, M., Taylor, K., Fineman, M., et al., 2005. Exenatide improves glycemic control and reduces body weight in subjects with type 2 diabetes: a dose-ranging study. Diabetes Technology and Therapeutics 7:467-477.

[885] Edwards, C.M., Stanley, S.A., Davis, R., Brynes, A.E., Frost, G.S., Seal, L.J., et al., 2001. Exendin-4 reduces fasting and postprandial glucose and decreases energy intake in healthy volunteers. American Journal of Physiology Endocrinology and Metabolism 281:E155-E161.

[886] Ratner, R.E., Maggs, D., Nielsen, L.L., Stonehouse, A.H., Poon, T., Zhang, B., et al., 2006. Long-term effects of exenatide therapy over 82 weeks on glycaemic control and weight in over-weight metformin-treated patients with type 2 diabetes mellitus. Diabetes Obesity and Metabolism 8:419-428.
[887] Toft-Nielsen, M.B., Madsbad, S., Holst, J.J., 1999. Continuous subcutaneous infusion of glucagon-like peptide 1 lowers plasma glucose and reduces appetite in type 2 diabetic patients. Diabetes Care 22:1137-1143.

[888] Aroda, V.R., DeYoung, M.B., 2011. Clinical implications of exenatide as a twice-daily or once-weekly therapy for type 2 diabetes. Postgraduate Medicine 123:228-238.

[889] Thorkildsen, C., Neve, S., Larsen, B.D., Meier, E., Petersen, J.S., 2003. Glucagon-like peptide 1 receptor agonist ZP10A increases insulin mRNA expression and prevents diabetic progression in $\mathrm{db} / \mathrm{db}$ mice. Journal of Pharmacology and Experimental Therapeutics 307:490-496.

[890] McCarty, D., Coleman, M., Boland, C.L., 2017. Lixisenatide: a new daily GLP1 Agonist for type 2 diabetes management. The Annals of Pharmacotherapy 51:401-409.

[891] Finan, B., Clemmensen, C., Muller, T.D., 2015. Emerging opportunities for the treatment of metabolic diseases: Glucagon-like peptide- 1 based multiagonists. Molecular and Cellular Endocrinology 418(Pt 1):42-54.

[892] Ratner, R.E., Rosenstock, J., Boka, G., Investigators, D.R.I.S., 2010. Dosedependent effects of the once-daily GLP-1 receptor agonist lixisenatide in patients with Type 2 diabetes inadequately controlled with metformin: a randomized, double-blind, placebo-controlled trial. Diabetic Medicine 27: 1024-1032.

[893] Jacobsen, L.V., Flint, A., Olsen, A.K., Ingwersen, S.H., 2016. Liraglutide in Type 2 Diabetes Mellitus: Clinical Pharmacokinetics and Pharmacodynamics. Clinical Pharmacokinetics 55:657-672.

[894] Agerso, H., Jensen, L.B., Elbrond, B., Rolan, P., Zdravkovic, M., 2002. The pharmacokinetics, pharmacodynamics, safety and tolerability of NN2211, a new long-acting GLP-1 derivative, in healthy men. Diabetologia 45:195-202.

[895] O'Neil, P.M., Birkenfeld, A.L., McGowan, B., Mosenzon, O., Pedersen, S.D., Wharton, S., et al., 2018. Efficacy and safety of semaglutide compared with liraglutide and placebo for weight loss in patients with obesity: a randomised, double-blind, placebo and active controlled, dose-ranging, phase 2 trial. Lancet 392:637-649.

[896] Lau, J., Bloch, P., Schaffer, L., Pettersson, I., Spetzler, J., Kofoed, J., et al., 2015. Discovery of the Once-Weekly Glucagon-Like Peptide-1 (GLP-1) Analogue Semaglutide. Journal of Medicinal Chemistry 58:7370-7380.

[897] Gotfredsen, C.F., Molck, A.M., Thorup, I., Nyborg, N.C., Salanti, Z., Knudsen, L.B., et al., 2014. The human GLP-1 analogs liraglutide and semaglutide: absence of histopathological effects on the pancreas in nonhuman primates. Diabetes 63:2486-2497.

[898] Pratley, R.E., Aroda, V.R., Lingvay, I., Ludemann, J., Andreassen, C. Navarria, A., et al., 2018. Semaglutide versus dulaglutide once weekly in patients with type 2 diabetes (SUSTAIN 7): a randomised, open-label, phase 3b trial. Lancet Diabetes Endocrinology 6:275-286.

[899] Marso, S.P., Bain, S.C., Consoli, A., Eliaschewitz, F.G., Jodar, E., Leiter, L.A., et al., 2016. Semaglutide and Cardiovascular Outcomes in Patients with Type 2 Diabetes. New England Journal of Medicine 375:1834-1844.

[900] Glaesner, W., Vick, A.M., Millican, R., Ellis, B., Tschang, S.H., Tian, Y., et al., 2010. Engineering and characterization of the long-acting glucagon-like peptide-1 analogue LY2189265, an Fc fusion protein. Diabetes Metabolism in Research Review 26:287-296.

[901] Barrington, P., Chien, J.Y., Tibaldi, F., Showalter, H.D., Schneck, K., Ellis, B., 2011. LY2189265, a long-acting glucagon-like peptide-1 analogue, showed a dose-dependent effect on insulin secretion in healthy subjects. Diabetes Obesity and Metabolism 13:434-438.

[902] Dungan, K.M., Povedano, S.T., Forst, T., Gonzalez, J.G., Atisso, C., Sealls, W., et al., 2014. Once-weekly dulaglutide versus once-daily liraglutide in metformin-treated patients with type 2 diabetes (AWARD-6): a randomised, open-label, phase 3, non-inferiority trial. Lancet 384:1349-1357.

[903] Bush, M.A., Matthews, J.E., De Boever, E.H., Dobbins, R.L., Hodge, R.J., Walker, S.E., et al., 2009. Safety, tolerability, pharmacodynamics and 
pharmacokinetics of albiglutide, a long-acting glucagon-like peptide-1 mimetic, in healthy subjects. Diabetes Obesity and Metabolism 11:498-505.

[904] Alfaris, N., Minnick, A.M., Hopkins, C.M., Berkowitz, R.I., Wadden, T.A., 2015. Combination phentermine and topiramate extended release in the management of obesity. Expert Opinion on Pharmacotherapy 16:12631274.

[905] Gadde, K.M., Allison, D.B., Ryan, D.H., Peterson, C.A., Troupin, B., Schwiers, M.L., et al., 2011. Effects of low-dose, controlled-release, phentermine plus topiramate combination on weight and associated comorbidities in overweight and obese adults (CONQUER): a randomised, placebocontrolled, phase 3 trial. Lancet 377:1341-1352.

[906] Diamant, M., Nauck, M.A., Shaginian, R., Malone, J.K., Cleall, S., Reaney, M., et al., 2014. Glucagon-like peptide 1 receptor agonist or bolus insulin with optimized basal insulin in type 2 diabetes. Diabetes Care 37:2763-2773.

[907] Eng, C., Kramer, C.K., Zinman, B., Retnakaran, R., 2014. Glucagon-like peptide-1 receptor agonist and basal insulin combination treatment for the management of type 2 diabetes: a systematic review and meta-analysis. Lancet 384:2228-2234.

[908] Goldenberg, R.M., Berard, L., 2018. Adding prandial GLP-1 receptor agonists to basal insulin: a promising option for type 2 diabetes therapy. Current Medical Research and Opinion 34:1-10.

[909] Yang, W., Cai, X., Gao, X., Chen, Y., Chen, L., Ji, L., 2018. Addition of dipeptidyl peptidase-4 inhibitors to insulin treatment in type 2 diabetes patients: A meta-analysis. Journal of Diabetes Investigation 9:813-821.

[910] Nauck, M.A., Kahle, M., Baranov, O., Deacon, C.F., Holst, J.J., 2017. Addition of a dipeptidyl peptidase-4 inhibitor, sitagliptin, to ongoing therapy with the glucagon-like peptide-1 receptor agonist liraglutide: A randomized controlled trial in patients with type 2 diabetes. Diabetes Obesity and Metabolism 19: 200-207.

[911] Violante, R., Oliveira, J.H., Yoon, K.H., Reed, V.A., Yu, M.B., Bachmann, O.P., et al., 2012. A randomized non-inferiority study comparing the addition of exenatide twice daily to sitagliptin or switching from sitagliptin to exenatide twice daily in patients with type 2 diabetes experiencing inadequate glycaemic control on metformin and sitagliptin. Diabetic Medicine 29:e417e424.

[912] Kanoski, S.E., Ong, Z.Y., Fortin, S.M., Schlessinger, E.S., Grill, H.J., 2015 Liraglutide, leptin and their combined effects on feeding: additive intake reduction through common intracellular signalling mechanisms. Diabetes Obesity and Metabolism 17:285-293.

[913] Muller, T.D., Sullivan, L.M., Habegger, K., Yi, C.X., Kabra, D., Grant, E., et al., 2012. Restoration of leptin responsiveness in diet-induced obese mice using an optimized leptin analog in combination with exendin-4 or FGF21. Journal of Peptide Science 18:383-393.

[914] Bello, N.T., Kemm, M.H., Ofeldt, E.M., Moran, T.H., 2010. Dose combinations of exendin-4 and salmon calcitonin produce additive and synergistic reductions in food intake in nonhuman primates. American Journal of Physiology Regulatory Integrative and Comparative Physiology 299:R945-R952.

[915] Neary, N.M., Small, C.J., Druce, M.R., Park, A.J., Ellis, S.M., Semjonous, N.M., et al., 2005. Peptide YY3-36 and glucagon-like peptide17-36 inhibit food intake additively. Endocrinology 146:5120-5127.

[916] Trevaskis, J.L., Sun, C., Athanacio, J., D’Souza, L., Samant, M., Tatarkiewicz, K., et al., 2015. Synergistic metabolic benefits of an exenatide analogue and cholecystokinin in diet-induced obese and leptin-deficient rodents. Diabetes Obesity and Metabolism 17:61-73.

[917] Baruah, M.P., Kalra, S., 2012. The novel use of GLP-1 analogue and insulin combination in type 2 diabetes mellitus. Recent Patents on Endocrine Metabolic and Immune Drug Discovery 6:129-135.

[918] Bech, E.M., Voldum-Clausen, K., Pedersen, S.L., Fabricius, K., Rudkjaer, L.C.B., Hansen, H.H., et al., 2019. Adrenomedullin and glucagonlike peptide- 1 have additive effects on food intake in mice. Biomedicine and Pharmacotherapy 109:167-173.
[919] Decara, J., Rivera, P., Arrabal, S., Vargas, A., Serrano, A., Pavon, F.J., et al., 2018. Cooperative role of the glucagon-like peptide-1 receptor and beta3adrenergic-mediated signalling on fat mass reduction through the downregulation of PKA/AKT/AMPK signalling in the adipose tissue and muscle of rats. Acta Physiologica (Oxford) 222:e13008.

[920] Bojanowska, E., Radziszewska, E., 2011. Combined stimulation of glucagonlike peptide-1 receptor and inhibition of cannabinoid CB1 receptor act synergistically to reduce food intake and body weight in the rat. Journal of Physiology and Pharmacology 62:395-402.

[921] Jouihan, H., Will, S., Guionaud, S., Boland, M.L., Oldham, S., Ravn, P., et al., 2017. Superior reductions in hepatic steatosis and fibrosis with coadministration of a glucagon-like peptide- 1 receptor agonist and obeticholic acid in mice. Molecular Metabolism 6:1360-1370.

[922] Day, J.W., Ottaway, N., Patterson, J.T., Gelfanov, V., Smiley, D., Gidda, J., et al., 2009. A new glucagon and GLP-1 co-agonist eliminates obesity in rodents. Nature Chemical Biology 5:749-757.

[923] Pocai, A., Carrington, P.E., Adams, J.R., Wright, M., Eiermann, G., Zhu, L., et al., 2009. Glucagon-like peptide 1/glucagon receptor dual agonism reverses obesity in mice. Diabetes 58:2258-2266.

[924] Evers, A., Haack, T., Lorenz, M., Bossart, M., Elvert, R., Henkel, B., et al., 2017. Design of novel exendin-based dual glucagon-like peptide 1 (GLP1)/glucagon receptor agonists. Journal of Medicinal Chemistry 60:42934303.

[925] Clemmensen, C., Finan, B., Muller, T.D., DiMarchi, R.D., Tschop, M.H., Hofmann, S.M., 2019. Emerging hormonal-based combination pharmacotherapies for the treatment of metabolic diseases. Nature Reviews Endocrinology 15:90-104.

[926] Ambery, P., Parker, V.E., Stumvoll, M., Posch, M.G., Heise, T., PlumMoerschel, L., et al., 2018. MEDI0382, a GLP-1 and glucagon receptor dual agonist, in obese or overweight patients with type 2 diabetes: a randomised, controlled, double-blind, ascending dose and phase 2a study. Lancet 391: 2607-2618.

[927] Tillner, J., Posch, M.G., Wagner, F., Teichert, L., Hijazi, Y., Einig, C., et al., 2019. A novel dual glucagon-like peptide and glucagon receptor agonist SAR425899: Results of randomized, placebo-controlled first-in-human and first-in-patient trials. Diabetes Obesity and Metabolism 21:120-128.

[928] Nauck, M.A., Bartels, E., Orskov, C., Ebert, R., Creutzfeldt, W., 1993. Additive insulinotropic effects of exogenous synthetic human gastric inhibitory polypeptide and glucagon-like peptide-1-(7-36) amide infused at nearphysiological insulinotropic hormone and glucose concentrations. The Journal of Cinical Endocrinology and Metabolism 76:912-917.

[929] Finan, B., Muller, T.D., Clemmensen, C., Perez-Tilve, D., DiMarchi, R.D., Tschop, M.H., 2016. Reappraisal of GIP pharmacology for metabolic diseases. Trends in Molecular Medicine 22:359-376.

[930] Irwin, N., Flatt, P.R., 2009. Therapeutic potential for GIP receptor agonists and antagonists. Best Practice and Research Clinical Endocrinology and Metabolism 23:499-512.

[931] Irwin, N., Gault, V., Flatt, P.R., 2010. Therapeutic potential of the original incretin hormone glucose-dependent insulinotropic polypeptide: diabetes, obesity, osteoporosis and Alzheimer's disease? Expert Opinion on Investigational Drugs 19:1039-1048.

[932] Mroz, P.A., Finan, B., Gelfanov, V., Yang, B., Tschop, M.H., DiMarchi, R.D., et al., 2019. Optimized GIP analogs promote body weight lowering in mice through GIPR agonism not antagonism. Molecular Metabolism 20:51-62.

[933] Killion, E.A., Wang, J., Yie, J., Shi, S.D., Bates, D., Min, X., et al., 2018. Antiobesity effects of GIPR antagonists alone and in combination with GLP-1R agonists in preclinical models. Science Translational Medicine 10.

[934] Pathak, V., Gault, V.A., Flatt, P.R., Irwin, N., 2015. Antagonism of gastric inhibitory polypeptide (GIP) by palmitoylation of GIP analogues with $\mathrm{N}$ - and Cterminal modifications improves obesity and metabolic control in high fat fed mice. Molecular and Cellular Endocrinology 401:120-129. 
[935] Norregaard, P.K., Deryabina, M.A., Tofteng Shelton, P., Fog, J.U., Daugaard, J.R., Eriksson, P.0., et al., 2018. A novel GIP analogue, ZP4165, enhances glucagon-like peptide-1-induced body weight loss and improves glycaemic control in rodents. Diabetes Obesity and Metabolism 20:60-68.

[936] Irwin, N., Flatt, P.R., 2015. New perspectives on exploitation of incretin peptides for the treatment of diabetes and related disorders. World Journal of Diabetes 6:1285-1295.

[937] Pathak, N.M., Pathak, V., Gault, V.A., McClean, S., Irwin, N., Flatt, P.R., 2018. Novel dual incretin agonist peptide with antidiabetic and neuroprotective potential. Biochemical Pharmacology 155:264-274.

[938] Coskun, T., Sloop, K.W., Loghin, C., Alsina-Fernandez, J., Urva, S., Bokvist, K.B., et al., 2018. LY3298176, a novel dual GIP and GLP-1 receptor agonist for the treatment of type 2 diabetes mellitus: From discovery to clinical proof of concept. Molecular Metabolism 18:3-14.

[939] Frias, J.P., Nauck, M.A., Van, J., Kutner, M.E., Cui, X., Benson, C., et al., 2018. Efficacy and safety of LY3298176, a novel dual GIP and GLP-1 receptor agonist, in patients with type 2 diabetes: a randomised, placebo-controlled and active comparator-controlled phase 2 trial. Lancet 392:2180-2193.

[940] Cassidy, R.S., Irwin, N., Flatt, P.R., 2008. Effects of gastric inhibitory polypeptide (GIP) and related analogues on glucagon release at normo- and hyperglycaemia in Wistar rats and isolated islets. Biological Chemistry 389: 189-193.

[941] Gault, V.A., Bhat, V.K., Irwin, N., Flatt, P.R., 2013. A novel glucagon-like peptide-1 (GLP-1)/glucagon hybrid peptide with triple-acting agonist activity at glucose-dependent insulinotropic polypeptide, GLP-1, and glucagon receptors and therapeutic potential in high fat-fed mice. Journal of Biological Chemistry 288:35581-35591.

[942] Fosgerau, K., Jessen, L., Lind Tolborg, J., Osterlund, T., Schaeffer Larsen, K., Rolsted, K., et al., 2013. The novel GLP-1-gastrin dual agonist, ZP3022, increases beta-cell mass and prevents diabetes in $\mathrm{db} / \mathrm{db}$ mice. Diabetes Obesity and Metabolism 15:62-71.

[943] Hasib, A., Ng, M.T., Tanday, N., Craig, S.L., Gault, V.A., Flatt, P.R., et al., 2019. Exendin-4(Lys(27) PAL)/gastrin/xenin-8-Gln: A novel acylated GLP-1/ gastrin/xenin hybrid peptide that improves metabolic status in obesediabetic (ob/ob) mice. Diabetes Metabolism in Research Review 35:e3106.
[944] Hasib, A., Ng, M.T., Khan, D., Gault, V.A., Flatt, P.R., Irwin, N., 2018. Characterisation and antidiabetic utility of a novel hybrid peptide, exendin-4/ gastrin/xenin-8-GIn. European Journal of Pharmacology 834:126-135.

[945] Trevaskis, J.L., Mack, C.M., Sun, C., Soares, C.J., D’Souza, L.J., Levy, O.E., et al., 2013. Improved glucose control and reduced body weight in rodents with dual mechanism of action peptide hybrids. PLoS One 8:e78154.

[946] Hornigold, D.C., Roth, E., Howard, V., Will, S., Oldham, S., Coghlan, M.P., et al., 2018. A GLP-1:CCK fusion peptide harnesses the synergistic effects on metabolism of CCK-1 and GLP-1 receptor agonism in mice. Appetite 127: $334-340$.

[947] Irwin, N., Pathak, V., Flatt, P.R., 2015. A Novel CCK-8/GLP-1 hybrid peptide exhibiting prominent insulinotropic, glucose-lowering, and satiety actions with significant therapeutic potential in high-fat-fed mice. Diabetes 64: 2996-3009.

[948] Talukdar, S., Zhou, Y., Li, D., Rossulek, M., Dong, J., Somayaji, V., et al., 2016. A long-acting FGF21 molecule, PF-05231023, decreases body weight and improves lipid profile in non-human primates and type 2 diabetic subjects. Cell Metabolism 23:427-440.

[949] Chodorge, M., Celeste, A.J., Grimsby, J., Konkar, A., Davidsson, P., Fairman, D., et al., 2018. Engineering of a GLP-1 analogue peptide/antiPCSK9 antibody fusion for type 2 diabetes treatment. Scientific Reports 8: 17545.

[950] Jain, M., Carlson, G., Cook, W., Morrow, L., Petrone, M., White, N.E., et al., 2019. Randomised, phase 1, dose-finding study of MEDI4166, a PCSK9 antibody and GLP-1 analogue fusion molecule, in overweight or obese patients with type 2 diabetes mellitus. Diabetologia 62:373-386.

[951] Ammala, C., Drury 3rd, W.J., Knerr, L., Ahlstedt, I., Stillemark-Billton, P., Wennberg-Huldt, C., et al., 2018. Targeted delivery of antisense oligonucleotides to pancreatic beta-cells. Science Advances 4:eaat3386.

[952] Schwenk, R.W., Baumeier, C., Finan, B., Kluth, O., Brauer, C., Joost, H.G., et al., 2015. GLP-1-oestrogen attenuates hyperphagia and protects from beta cell failure in diabetes-prone New Zealand obese (NZO) mice. Diabetologia 58:604-614.

[953] Tiano, J.P., Tate, C.R., Yang, B.S., DiMarchi, R., Mauvais-Jarvis, F., 2015. Effect of targeted estrogen delivery using glucagon-like peptide-1 on insulin secretion, insulin sensitivity and glucose homeostasis. Scientific Reports 5: 10211. 